Furopean

Surgical Research

Abstracts

Eur Surg Res 2010;45:158-307

DOI: $10.1159 / 0000321283$

The abstracts are available free online at www.karger.com/doi/10.1159/0000321283

\title{
23rd National Congress of the Italian Society of Young Surgeon (SPIGC)
}

June 20-23, 2010

Forlì
Guest Editors

Davide Cavaliere, Forlì Niccolò Daddi, Perugia

Associated Editors

Gabriella Teresa Capolupo, Rome

Gabriele Galatá, Rome

Piero Sorrentino, Naples 


\section{Contents}

Introduction $\quad 160$

From The Patient's Perspective $\quad 160$

$\begin{array}{ll}\text { Anaesthesia } & 161\end{array}$

Breast Surgery $\quad 161$

Cardiac Surgery 162

General Surgery $\quad 165$

Laparoscopic Surgery $\quad 203$

Oncologic Surgery $\quad 210$

Plastic Surgery $\quad 227$

Thoracic Surgery $\quad 241$

Vascular Surgery $\quad 258$

Day Surgery $\quad 268$

Emergency Surgery $\quad 270$

Endocrine Surgery $\quad 272$

ENT $\quad 275$

Experimental Surgery $\quad 281$

Gynecological Surgery $\quad 281$

Maxillofacial Surgery $\quad 283$

Obesity Surgery $\quad 290$

Orthopedic and Trauma Surgery $\quad 292$

Pediatric Surgery $\quad 298$

Transplantation $\quad 299$

Urology $\quad 300$

$\begin{array}{ll}\text { Author Index } & 301\end{array}$ 


\section{European \\ Surgical Research}

\section{Introduction}

Joseph P. Vacanti

John Homans Professor of Surgery, Harvard Medical School, Surgeon in Chief, Massachusetts General Hospital for Children, Deputy Director, Center for Regenerative Medicine, Massachusetts, General Hospital, Boston, MA, USA

The field of Tissue Engineering emerged from the clinical need of surgeons to have better methods of reconstruction for children and adults in whom tissue has been destroyed by disease, trauma, and congenital anomalies. The concepts are fundamentally different from what has gone before in that the premise is to create or fabricate living structures for human replacement rather that inanimate devices or substitute structures such as a colon in place of an esophagus, or a toe in place of a finger. In the case of vital organ replacement, the thesis is that new vital organs can be constructed on demand instead of transplanting existing organs in such short supply. It also offers the possibility of eliminating immune mismatch, recognition, and rejection by using the patient's own cells or immunologically inert cells. The fundamental principles to create useful living tissue have been elucidated over the last 25 years. The recognition of the need for sufficient mass transfer of nutrients, oxygen, and waste removal for large masses of living cells has led to several strategies of design. The use of modern engineering tools such as computational fluid dynamics, new innovations in materials science and chemical engineering, and rapid progress in stem cell science have all contributed to enormous progress in the field. Currently, several tissue engineered structures are available for human therapy either as approved devices or in clinical trials. Importantly, the advance of technology offers hope in many conditions currently untreatable, including the solution to the organ donor shortage. Young surgeons engaged in this new science will be important contributors on the road to human therapy.

\section{From The Patient's Perspective}

Sylvie Ménard

Fondazione IRCCS Istituto Nazionale tumori, Milan, Italy

Recent development of cancer therapies is often considered with enthusiasm for their capability to increase survival without adding toxicity. Indeed, in comparison with chemotherapies in general, no alopecia and vomiting are generally induced by the targeted drugs. In my 40-years experience in research on breast carcinomas, I have participated to the development of different anti-breast carcinoma therapeutic drugs as trastuzumab, bevacizumab and different tyrosin-kinase inhibitors, having the possibility to evaluate the quality of life impact of the new drugs. I was convinced that the development of new drugs with low toxicity was the way to improve the quality of life of the patients. Few years ago, I moved from the researcher status to a cancer patient status with a myeloma diagnosis. Looking at the new therapies from the patient point of view, I realized that, first of all, the 'toxicity' of the drugs is not directly related to the quality of life since the thought of having a cancer is more 'toxic' than any drugs. Second, 'toxicity' is not only a physical damage, but is principally related to the possibility for the patient to perform his life as normal as possible. The way of delivery of the new drugs (in hospital as i.v. injection versus at home as pills), the frequency of treatment if delivered in hospital, the waiting list for any examinations, the lack of information of what is happening are all situations that condition the quality of life much more than the chemotherapy- associated side effect. Because the new therapies increase the patient survival without definitively cure the disease, this extra time given to the patient must be lived with the maximum of serenity. This is possible only if the communication between the patient and the sanitary officers, including medical doctors, nurses, and even administrative personnel is of quality. Not only therapies can help in curing cancer patients but also words depending how and when they are said or written. For example, any information on a poor prognosis is detrimental for the parent. Nevertheless, doctors must find the words to realistically explain to the patient the possibilities to fight the disease, leaving always some hope. The presentation will summarize the different steps of the disease from a psychological point of view and discuss the patient expectations. The differences between the rights of the patients seen from the healthy people point of view and those seen from the patients point of view will be presented.

\begin{tabular}{ll}
\hline KARGER & $\oplus$ 2010 S. Karger AG, Basel \\
Fax +41613061234 & $0014-312 X / 10 / 0454-0158 \$ 26.00 / 0$ \\
$\begin{array}{l}\text { E-Mail karger@karger.ch } \\
\text { www.karger.com }\end{array}$ & $\begin{array}{l}\text { Accessible online at: } \\
\text { www.karger.com/esr }\end{array}$
\end{tabular}




\section{Furopean \\ Surgical Research}

\section{Anaesthesia}

1

\section{Anaphylactic Shock After Neuromuscolar Blocker Administration}

\author{
1. Scaioli', E. Piraccini ${ }^{1}$, D. Cavaliere ${ }^{2}$, R. Righetti ${ }^{1}$, \\ C. Rotondo', F. Avino1, G. Gambale1 \\ ${ }^{1}$ Department of Anesthesia and Intensive Care, Morgagni \\ Pierantoni Hospital, Forlì, Italy, ${ }^{2}$ Department of General \\ Surgery, Morgagni Pierantoni Hospital, Forlì, Italy
}

Introduction: The risk of developing an anaphylactic reaction during anesthesia has been estimated at 1:4.600 procedures and neuromuscolar blockers are the leading cause.

Methods: A 37 y.o. man $(B M I=37,9)$ was scheduled for thymectomy. He had no history of allergy and he never received a general anesthesia (GA).

After the GA induction patient's conditions were stable, after Atracurium administration an important cutaneous rush was observed, heart rate (HR) increased to $120 \mathrm{bpm}$, BP decreased to $50 / 26 \mathrm{mmHg}$ and oximetry value decreased to $74 \%$. Wheezing and high airway pressure raised.

Anaphylactic shock was treated with boluses and continuous infusion of epinephrine, the patient received also corticosteroids. Cardiovascular variables improved (BP 80/50 mmHg, HR 100 bpm, Sp O2=89\%). Sevoflurane administration was stared in an O2/air mix.

Results: The patient was sedated during the event since his cardiovascular and respiratory conditions completely recovered 90 minutes after the event. The surgery was postponed and the patient was moved to the ICU.

Conclusion: The diagnosis of anaphylactic reaction is clinical, biochemical tests can confirm the event.

A prior exposure to the drug is not necessary as the reaction can be the result of cross-reactivity to the ammonium ion found in other products as drugs or foods.

An early approach is mandatory to avoid serious consequences.

\section{Breast Surgery}

\section{2 \\ Initial Experience in Sentinel Lymph Node (SLN) Detection by Fluorescence Lymph Angiography Technique}

T. Fogacci, D. Samorani, A. Manzo, G. Frisoni, M. Montesi
At the Rimini AUSL Senology Functional Unit the Indocyanine green (ICG) was tested:the ICG,once in human body, can be detected by an infrared video-camera. This equipment, PDE ("Photo Dynamic Eye") enables to discover the SLN after 5-15 minutes,following lymph flow in real time,once the marker is injected around the cancer area or under the aureola. ICG is a lightweight molecule that binds to plasma proteins almost immediately without modifying their dimensions and becomes fluorescent in the near infrared range. Infrared light and video-camera allow to monitor the flow till the lymph node.

Results: This technique,always in combination with radioactive technetium, was used in 4 cases: in three of them the SLN was detected and the ICG use allowed to find the SLN rapidly perfectly in line with radio-active technique. In the fourth case, when the SLN could not be detected, neither the pre-surgical scintigraphy was successful and the axillary dissection had to be made.

Discussion: The use of PDE would allow a remarkable economic saving due to: elimination of radio-active material; less discomfort for patients who do no longer need attending nuclear medicine department. This experience allows to say that this technique is worth further tests, always in combination with the traditional technique, followed by randomized studies. Most likely, should further studies confirm our first findings, the above technique could be adopted in SLN detection, instead of radio-active marker, in specific cases.

\section{KARGER}

(c) 2010 S. Karger AG, Basel

Fax +41613061234

E-Mail karger@karger.ch

www.karger.com 


\section{3}

\section{New Techniques in Breast Cancer Surgery: A Multicentric Regional Study}

\author{
A. dell' Antonio, M.Giuricin, P. Tarchi, M. Borrelli, \\ G. Giacomel, U. Ginanneschi \\ Department of General surgery, University of Trieste, Italy
}

Background: The incidence of upper arm limphedema and axillary seroma formation after limphadenectomy for breast cancer varies from 10 to $38 \%$ in the literature.

Materials and Methods: Aim of the study is to demonstrate that Armonic Scalpel (AS) can reduce the incidence of those complications due to the low coagulative temperature of this instrument reduce the necrosis and subsequent inflammation.

We started a multicentric prospective randomizied trial, that was analyzed by the "split-plot design" method. From 2008 to 2010 80 patients from 5 different Friuli Venezia Giulia centers (Trieste, Udine, Gorizia, Tolmezzo, San Daniele) were enrolled. Median age was 63 (range 32-90). The Patients had a breast cancer staged I to IIIA according to the TMN classification, all histologies and gradings were included. A mastectomy or quadrantectomy were performed, in association with an axillary limphadenectomy and a axillary cavity drain placement. Patients were divided in 2 groups: group A 40 patients, operated using the AS, group B 40 patients using Bipolar scalpel or Electrocautery . Based on clinical evidence, in group A the incidence of all early and long-term complications was smaller.

The mean volume of the drain in group A was $183 \mathrm{cc}$ and in group B $523 \mathrm{cc}$, the mean length of the lymphatic drainage was 12 days in group A and 15 in group B. These two parameters were statistically relevant (Wilcoxon test $\mathrm{p}<0.001$ ).

Conclusions: Our experience was based on few parameters but was statistically relevant and it confirms that the use of the Armonic Scalpel in the breast cancer surgery is effective.

\section{4 \\ Staging Multicentric Breast Cancer by Sentinel Lymph Node Biopsy: The Experience of the European Institute of Oncology \\ F. Soggiu, G. Lissidini, S. Musmeci, P. Veronesi, O. Gentilini \\ Division of Senology, European Institute of Oncology, \\ Milano, Italy}

Introduction: Sentinel lymph node biopsy (SLNB) is an established procedure for nodal staging in breast cancer patients with a clinically negative axilla. The aim of this study is to report our experience with SLNB in patients with multicentric breast cancer.

Materials and Methods: Between 2001 and 2009, 346 patients with unilateral multicentric breast cancer and a clinically negative axilla underwent SLNB. Axillary dissection was performed only in case of positive sentinel node (SLN).
Results: The median duration of follow up was 57.4 months (17.1-140.1). Median age was 48 years. Median size of the largest tumor was $1.8 \mathrm{~cm}$. Mean number of removed SLNs was 1.68. The intra-operatory examination found 139 negative SLN and 207 positive SLN (13 ITC, 54 micrometastases and 140 macrometastases). In $23.6 \%$ of patients with positive SLN, this was the only positive lymph node. During follow-up, 59 events occurred: 24 local recurrences, 3 controlateral tumors and 25 distant metastases. Thirteen patients $(3.8 \%)$ died during follow-up. Nine events were observed in patients with negative SLN (6.1\%), with three cases of axillary reappearance of the disease $(2 \%)$.

Conclusions: Axillary nodal recurrence is reasonably low in patients with multicentric breast cancer and negative sentinel node. Staging multicentric breast cancer by SLNB is our routine policy in patients with clinically negative axilla.

\section{Cardiac Surgery}

5

\section{The Frozen Elephant Trunk Technique with the E-vita Open Prosthesis}

\author{
A. Armaro*, L. Di Marco, A. Leone, P. Berretta, J. Pavicevic, \\ R. Di Bartolomeo.
}

Department of Cardiac Surgery, S. Orsola-Malpighi

Hospital, University of Bologna, Bologna, Italy

Introduction: The most recent development of the classic elephant trunk technique, the 'frozen elephant trunk' technique, represents the combination of an endovascular approach with a conventional surgical treatment for a hybrid approach.

Materials and Methods: Between January 2007 and December 2009, 67 patients with extensive pathology of the thoracic aorta have been treated with the frozen elephant trunk in our Institution.

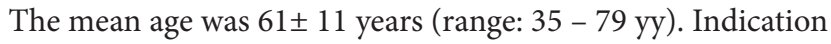
for surgery included: chronic degenerative aneurysm $(n=22$; $32.8 \%)$ acute type A dissection $(n=5 ; 7.5 \%)$, acute type B dissection $(\mathrm{n}=1 ; 1.5 \%)$, chronic type A dissection $(\mathrm{n}=30 ; 44.8 \%)$; chronic type $\mathrm{B}$ dissection $(\mathrm{n}=9 ; 13.4 \%)$.

Results: Hospital mortality was $13.4 \%(n=9)$; Postoperatively, 7 patients presented major neurologic complications such as coma $(n=5 ; 7.4 \%)$, paraplegia $(n=2 ; 2.9 \%)$ and paraparesis $(n=3 ; 4.4 \%)$. Pulmonary complications requiring a ventilatory support longer than 72 hours occurred in 19 patients (28.3\%), renal insufficiency in $21(31.3 \%)$, gut ischemia in $4(5.9 \%)$, low output syndrome in 9 (13.4\%), and bleeding requiring a rethoracotomy in 8 (11.9\%).

Conclusions: In our experience the frozen elephant trunk allowed a single-stage treatment for extensive pathology of the thoracic aorta with encouraging short and mid-term results.

The frozen elephant trunk technique offers the potential of a curative single-stage procedure for patients with aortic arch and proximal descending aorta pathology. Similarly to the conven- 
tional elephant trunk, the hybrid-prosthesis provides a secure landing zone for additional stent-grafts or may be used in secondstage open procedures in patients with thoracoabdominal aortic aneurysms.
7

\section{Repair of Coronary Artery Perforation Following Angioplasty Using TachoSil Patches}

M. Celiento, G. Scioti, S. Pratali, U. Bortolotti

\section{6}

\section{Sternal Synthesis Using Acellular Electrospun PLLA/Hydroxyapatite Scaffold: Potential Applications in Cardiothoracic Surgery}

C. Spadaccio ${ }^{1}$, M. Lusini', A. Rainer ${ }^{2}$, S. De Porcellinis 3 , A. Casacalenda ${ }^{1}$, R. Barbato ${ }^{1}$, A. Montecalvo ${ }^{1}$, M. Chello', E. Covino

${ }^{1}$ University Campus Bio-Medico of Rome, Department of cardiovascular Science, Unit of Cardiovascular Surgery. Via Alvaro del Portillo 200, 00128 Rome, Italy

${ }^{2}$ University Campus Bio-Medico of Rome, CIR Center for Integrated Research, Laboratory of Chemistry and Biomaterials, Via Alvaro del Portillo 200, 00128 Rome, Italy ${ }^{3}$ Biomatica, srl. Via Francesco Flora 75, 00139 Rome, Italy

Introduction: In the current cardiothoracic surgery practice, the everyday used median sternotomy access is at the root of several complications. We tested the in vivo ability of a previously developed electrospun poly-L-lactide/hydroxyapatite (PLLA/HA) composite scaffold to promote bone repair in a rabbit model of median sternotomy, simply interposing the biomaterial at the fracture site and therefore exposing directly bone marrow to a biologically oriented functionalized scaffold.

Materials and Methods: An electrospinning equipment (DynaSpin, Biomatica, Italy) was used to produce PLLA/HA scaffolds. A median sternotomy model in NZW rabbits was used. PLLA/HA was interposed between the two sternal halves. Sternum was then routinely closed by means of figure- 8 technique. Serial CT scans were performed at 1,2,3,4 weeks. Micro CT scans and $\mathrm{H} \& \mathrm{E}$ staining of histological section were obtained.

Results: We obtained a successful repair of sternal fracture at week 2. The control group totally healed at week 4. PLLA/HA scaffold was in the most part absorbed by enzymatic degradation processes reliably related to wound healing. Histological evaluation demonstrated presence of newly formed bone trabeculae among scaffold fibers with only mild signs of inflammation.

Conclusions: Using this model, with the direct exposure of bone marrow, we developed a cell-free system able to exploit and boost endogenous repair and stem cell resources, guiding them toward tissue restoration.

PLLA/HA fibrous scaffolds could represent an interesting and effective aid to sternal repair, reducing time of healing and avoiding the use of autologous cell transplantation.
Introduction: Coronary perforation is a complication of PTCA, which could be treated by pecutaneus trials; alternatively, emergency CABG may be required. We present an alternative way to treat such complication.

Case Report: 70-year old female, with precordial pain referred to Emergency Room. Angiography showed crtical stenosis of left anterior descending (LAD) coronary artery. PTCA with direct stenting was performed; the procedure was complicated by coronary disruption treated by new covered stent with apparently good result. Later, hemodynamic instability with hypotension occurred. Echocardiography showed pericardial effusion; after pericardiocentesis control angiography showed a site of active coronary bleeding on the LAD, and was decided for surgery. Despite various efforts it was impossible to identify the primary site of bleeding hematoma, and it was decided to treat it conservatively. After unsuccessful essay with dry gauzes, two TachoSil ${ }^{\circledR}$ patch where positioned on the bleeding sites and pressed over the beating left ventricle for $\sim 10 \mathrm{~min}$. No more bleeding was observed and the chest was closed in the routine fashion. Six month Echo control showed no evidence of pericardial effusion.

Conclusion: We suggest this is an alternative use of TachoSil ${ }^{\circledR}$ patch which avoids the use of cardiopulmonary bypass and provides an effective method to deal with such complications.

\section{8}

\section{Surgical Anatomy of the Abnormal Aortic Arch with Vascular Ring: From Diagnosis to Management}

M. Massara, F. Benedetto, N. Pipitò, G. Carella, R. Donato, G. Di Bella, S. Lentini, F. Spinelli

CardioVascular and Thoracic Department, Policlinico G. Martino, University of Messina

Introduction: Aortic arch abnormalities are quite rare, and may be associated to vascular ring. Vascular rings occur with an incidence of $<0.2 \%$, and usually presents in infancy or childhood. Often a right aortic arch is present. A right sided aortic arch is defined as an aortic arch that crosses over the right mainstem bronchus instead of the usual left mainstem bronchus.

A vascular ring is a malformation where vascular branches encircle the trachea and esophagus.

Case Report: We present the case of a 37-year-old man with history of chest discomfort, progressive mild dyspnea on exertion, and intermittent dysphagia for solids and liquids. Chest X-ray showed right aortic arch. Angio MRI confirmed the right aortic 
arch, and showed the presence of an aberrant left subclavian artery encircling the esophagus and trachea (vascular ring).

Discussion: Symptoms due to vascular ring include stridor, wheezing, and dysphagia are due to compression of the trachea or esophagus. However, if compression is minimal, then vascular rings may be asymptomatic, and be diagnosed as incidental finding. Angiography is not really necessary to establish the diagnosis of a vascular ring. MRI represents a non invasive, non ionizing imaging test, useful in the diagnosis of a vascular ring, gives informations about the precise arch configuration, vascular branching patterns and exact reciprocity of anatomic structures, useful for future surgery.

Conclusion: The authors describe the different abnormalities affecting the aortic arch, together with the vascular ring. The diagnostic work-up with different imaging modalities is described. Surgical indications are defined.

\section{9 \\ Endovascular Treatment of Chronic Upper Extremity Ischemia After Bypass Surgery Failure}

N. Pipitò, F. Benedetto, G. Passari, G. De Caridi, S. Lentini, F. Spinelli

I: By our case we want to demonstrate that percutaneous angioplasty can be a valid technique, after a failing bypass surgery.

C.R.: 76 year,strong smoker with multiple comorbidities showing rest pain and multiple digital gangrene.

Initially we treated the patient with a brachio-radial bypass in VGS and then by a multiple tip digit amputations. At 2 years follow-up good healing of lesions.

The preliminary arteriography showed chronic occlusion of the ulnar artery. Radial artery was occluded at the source so we can see a singular section of it near the wrist, and insufficient collateral circulation in the palmar arch.

Endovascular recanalization of radial artery and palmar arch, and to accomplish it we practice, multiple angioplasty with low profile balloon. We dilatated the lesions of the radial artery and we continued with the same technique to reach the recanalization of the whole palmar arch.

D: Open surgery approach is the gold standard.

Spinelli, Roddy e Hughes surgical cases revealed good issues in the distance, with a percentage of primary patency of $80 / 85 \%$ and of secondary patency of $90 / 92 \%$, and a total limb salvage.

Gandini et al. report a case of ulnar artery revascularization through the palmar arch by extra stiff hidrofilic and low profile balloons. .(loop palmar technique)

Ferraresi reports an other case of multiple occlusions of radial artery treated by pta. An eight months follow-up demonstrates a complete lesions healing.

C: Endovascular technique can be a valid support to the traditional procedure thanks to the techniques and materials evolution.
10

\section{Emergency Surgical Treatment of Acute Superior Vena Cava Syndrome}

F. Benedetto, A. Modafferi, F. Stilo, M. Massara, A. Dattola, S. Lentini, F. Spinelli

CardioVascular and Thoracic Department, Policlinico G. Martino, University of Messina

Introduction: Superior Vena Cava syndrome is due to reduced venous return from the upper body to the right atrium. It is usually due to occlusion of superior vena cava. Extrinsic compression by neoplastic disease represents the most frequent cause. However, venous thrombosis may represent an other cause. Risk factors may predispose to acute thrombosis. This may represent a surgical emergency.

Case Report: A 55-year-old patient presented acutely unstable and with signs of superior vena cava (SVC) syndrome. He had history of polycythemia vera, and was on anticoagulation therapy with warfarin, and cytoreduction therapy. He had also pacing leads passing through the SVC.

Diagnosis using CT scan was reached, and the patient underwent emergency surgery. Surgical reconstruction of SVC was performed trough median sternotomy without cardiopulmonary bypass.

Discussion: Polycythemia vera is a myeloproliferative disorder. Patients with such pathology are at risk for thrombotic complications in both the arterial and venous systems. The presence of pacing leads on the right venous side of the heart represented an other risk factor. In acute thrombosis, diagnosis should be rapidly confirmed in the aim of a prompt treatment. CT scan researching particular signs of SVC syndrome represents a useful imaging test. Emergency treatment may be necessary.

Conclusion: The authors discuss on the diagnostic modalities and on the surgical options in this emergency clinical scenario.

\section{1}

\section{Mitroflow for the Aortic Valve Replacement: our Experience}

\author{
L. Careddu, A. Pantaleo, C. Savini, O. Rasovic, G. Folesani, \\ R. Di Bartolomeo
}

Introduction: Pericardial prostheses have been used as valvular substitutes since 1975, we analyze our population, from 2002 to 2008, of Mitroflow pericardial valves (Sorin Group).

Methods: Data from 131 patients with aortic valve replacement with bioprostheses between 2002 and 2008 were analyzed. Cumulative duration of follow-up was patient-years with a maximum duration of 4 years. The cause of the aortic valve lesions was stenosis in the majority of patients $(78,6 \%)$. Concomitant procedures were performed in 58 patients, especially CABG (46 patients). Statistical analysis was performed to identify valve- 
related events and mortality and independent determinants of outcome.

Results: Mean age was 76, $6 \pm 7,7$ years (male/female ratio 38/93). Hospital mortality was $7,6 \%$. Late mortality was $9,1 \%$ but with only 4 deaths for cardiac causes $(2,3 \%)$. The incidence of thromboembolic events was $3.8 \%$. Even prosthetic valve endocarditis was $3.8 \%$. Structural valve deterioration was $1.5 \%$. For the characteristic of this valve, especially due to the wide opening of the leaflets, we prefer implant this valve in patient with a small LVOT (mean 2,0 $\pm 0,1$ ). Preoperative mean aortic gradient was $49,6 \pm 18,6$ versus A long term follow-up mean aortic gradient $22,3 \pm 15,4$. The prostheses most frequently used in our Department were size $19 \mathrm{~mm}$ and $21 \mathrm{~mm}$. Advanced age, renal insufficiency and pulmonary disease were implicated in perioperative and postoperative comorbility.

Conclusions: After a mean follow-up of 2 years, the Mitroflow pericardial aortic valve seem to be a valuable choice in patients over 70 years old with a low rate of valve-related events and thromboembolic complications.

\section{General Surgery}

\section{2}

\section{Evaluation of Late Complications of Nissen Fundoplication Through Study of Swallowing in MRI: Advantages and Future Prospects}

\section{S. Ruggiero, G.T. Capolupo, I. Sansoni*, R. Alloni, R. Coppola}

Department of General Surgery, University Campus BioMedico di Roma, Rome, "Department of Radiology, University Campus Bio-Medico di Roma, Rome

Introduction: Laparoscopic Nissen fundoplication is the most common procedure used to control reflux symptoms in more than $90 \%$ of patients with gastroesophageal reflux disease. Literature reports an incidence of postoperative dysphagia of $30 \%$ caused by stenosis or herniation of gastric fundus. Barium esophagogram is the gold standard to evaluate postoperative complications. The aim of our study was to demonstrate the efficacy of MRI in providing a morphofunctional study of distal esophagus and esophagogastric junction (EGJ) by reproducing the physiological swallowing process.

Materials and Methods: We enrolled 59 patients affected by GERD, hiatal hernia or both. In 57 patients was performed a total fundoplication and in 2 cases a partial fundoplication, sec. Dor and sec. Toupet. Long-term clinical results were evaluated by administering the questionnaire GERD-HRQL developed by Velanovich. We screened patients with postoperative symptoms using MRI standardized sequences designed for the study of cardial region anatomy and pathophysiology of swallowing.
Results: We performed 3 interventions with laparotomic approach and 56 with laparoscopy, 2 converted to laparotomy. Dysphagia was observed in 29 patients (66\%): transitional in 17 (39\%, average duration 3-5.5 months), while in 12 patients $(27 \%)$ it was permanent. QoL improved after surgery: in $89 \%$ of patients the intervention allowed to control symptoms and reduced drug use.

Conclusions: The study of swallowing using magnetic resonance imaging is a valuable tool for assessing anatomy and physiology of esophagus-gastric junction in patients who experience postoperative dysphagia, understanding the mechanisms of surgical failure and accurately selecting the appropriate therapeutic approach.

13

Anal Fistula in Elderly: State of the Art

F. Pata, F. Ferrari, R. Sacco

General Surgery, "Magna Graecia” University, Catanzaro, Italy

Introduction: Anal fistula is a common disease in coloproctology and surgery is the only curative treatment. Then, inadequate surgery can lead to such disastrous results, such as faecal incontinence or complex and recurrent fistula.

Materials and Methods: Authors analyzed literature to detect the best therapy's choices in the treatment of anal fistula in elderly.

Results: Advance age improves risk of faecal incontinence after fistula-in-ano surgery.

Conclusion: The optimal goals of therapy are to eliminate fistula tract and while preserving sphincter function. In elderly patients the review of literature suggests the use of anorectal manometry to reveal preoperative poor sphincter function and the use of sphincter preserving surgery (f.e. seton placement or injection of fibrin glue), in order to obtain a rise in the postoperative functional outcome.

14

$\mathrm{Co}_{2}$-Laser Endolaryngeal Surgery as Treatment of Malignant and Benign Lesions: Results of a Monocentric Experience

G. Tenti, A. De Vito, P. Canzi, S. Zucchini

U.O. ORL Ospedale Pierantoni Morgagni- Forlì

Introduction: Results of $\mathrm{CO}_{2}$-laser endolaryngeal surgery as treatment of malignant and benign lesions at Pierantoni Morgagni Hospital ENT department.

Materials and Methods: Between January 2005 and January 201028 malignant and 50 benign laryngeal lesions have been treated by $\mathrm{CO}_{2}$-Laser endoscopic laryngeal technique. 
Concerning malignancies: we treated 7 Tis, 16 T1a,3 T1b,2 T2 by performing type 1 to type 4 cordectomy according with European Laringological Society classification.

Benign cases included: 3 Reinke's Oedema, 10 cordal cysts, 5 arytenoid granulomas, 4 papillomatosis, 17 vocal cord adduction paralysis, 9 cordal polyps, 1 congenital anterior web, and 1 epyglottoplasty. Each type of lesion required a specific laser application: mucosectomy with flap of free borders or vaporisation or lysis but always preserving the free margin of mucosa and lamina propria in order to maintain the mucosal wave.

Results: We observed one case of disease progression in T2 lesion due to paraglottic space invasion, that underwent to secondary total laryngectomy and post-operative radiotherapy. Between benign cases optimal functional and clinical outcomes was obtained: only one laryngeal papillomatosis needed for subsequent surgery.

Conclusions: Laser $\mathrm{CO}_{2}$ technique is a safe and less invasive surgery, results are comparable with traditional or open surgery in malignant or benign pathologies. It permits the preservation of laryngeal framework structures and assures a faster recovery of functions.

\section{5}

\section{Autologous Hemoconcentrates and Biomaterials in the Treatment of Immunohaematological Etiology Skin Ulcers: Case Report}

\section{E. Mastroluca, A. Police, F. Martinelli, C. Eberspacher, K. Llange Ayala, M.C. Tesori, D. Di Sabatino, L. Marcuccio}

Department of Surgical Sciences Policlinico Umbertol, University “La Sapienza”, Rome, Italy, Gruppo Italiano per la ricerca sui Bio-materiali Bio_match

Introduction: In patients with immunological diseases ulcers result difficult in healing, regarding the clinical stage of disease: the Authors describe the PRP use in a case report of their observation.

Materials and Methods: A 78 old year patient, suffering from essential thrombocytosis receiving Oncocarbide, was treated by us since 2008 because of two bilateral lower limb wounds occurred at different times. After WBP, autologous hemoconcentrates were used. PRP (gel) was obtained by multicomponent sampling through discontinuous flow cellular separator Haemonetics $\mathrm{MCS}+{ }^{\oplus}$. Using a circuit for collecting stem cells, we obtained a concentrate with a high number of lymphomonocyte, platelets and plasma (crioprecipitate). This one, mixed with CLP, rich in fibrinogen and in other extracellular matrix proteins, was divided in rates and stored at $-40^{\circ} \mathrm{C}$. PRP was placed straight upon the wound, on HYAFF scaffold or through infiltrations of margins.

Results: After four applications (24 days) one ulcer shows : $25 \%$ reduction, cleansed wound bed, substantial pain decrease, even during medications (from 8 to 3 PVS). After about two months of PRP gel applications, the wound bed superficialization allowed us to accomplish the treatment with exclusively hyaluronic acid products. We achieved full recovery in four months, after 18 outpatient medications. The contralateral wound is still in treatment.

Conclusions: PRP, applied both as gel and through infiltrations, give us the possibility to reach good results in the treatment of "difficult" ulcers, such as those due to hematological diseases.

\section{6}

\section{Autologous Leuko Platelet Concentrates in the Treatment of Vascular and Not Skin Ulcers: Our Experience}

\author{
M.C. Tesori, L. Palombi, A. Police, F. Lombardo, \\ E. Mastroluca, C. Di Cosimo, P. Florio, C. Eberspacher
}

Surgical Sciences Department (ex III Surgical Clinics) Policlinico Umberto I, Rome, UOS Chirurgia Generale ad Indirizzo Vascolare, Gruppo Italiano per la ricerca dei BioMateriali Bio-Match

Introduction: Regenerative medicine has created new possibilities in the treatment of otherwise difficult in healing ulcers. The Authors report a newer technique based on the use of leukoplatelet concentrates obtained from patients' plasma and then placed on wound (HYAFF scaffold and margins infiltration).

Materials and Method: Our experience (2008- 2010) consists of 28 patients with various etiology ulcers: 7 autoimmune, 13 ischemic ( 5 patients with diabetes), 7 phlebostatic, 1 pressure. PRP (gel) was obtained by multicomponent sampling through discontinuous flow cellular separator Haemonetics MCS $+{ }^{\oplus}$. Using a circuit for collecting stem cells, we obtained a concentrate with a high number of lymphomonocyte, platelets and plasma (crioprecipitate). This one, mixed with CLP, rich in fibrinogen and in other extracellular matrix proteins, was divided in rates and stored at $-40^{\circ} \mathrm{C}$.

Results: Dressings were performed at intervals of about 5-7 days. Three patients required a $6 / 8$ months treatment (thrombocytosis, post phlebitis syndrome), the others no more than 3 months.

Conclusions: The Authors confirm the effectiveness in promoting healing of ulcers and in reducing pain. After the development of indications, time and mode in the use of the preparation (effectiveness of fresh preparation, effectiveness in patients with immunological disease), Authors started a study using immunocytochemistry/ immunometric on biopsy performed in all the patients (preliminary observations on endothelial precursors). 
17

\section{Rectus Sheath Haematoma: Our Experience}

D.Amabile, M. Villa, F. Rulli, G. Tucci, M. Grande,

C. Dell'isola, G.M. Muzi, F. Andreoli, A.M. Farinon

Department of Surgery, University Hospital Tor Vergata, Rome

Background: Rectus sheath haematoma is considered a relatively rare condition that can be caused by local traumas, prolonged violent coughing, abdominal surgery and in some instances reported as spontaneous Oral anticogulant usage and congenital coagulation disorders are risk factors predisposing this clinical entity. $80 \%$ typically occurs in the lower abdominal quadrants (sub-umbilical) due to anatomical weaker support of the rectus muscle in this area. We report our series that includes 25 cases of rectus sheath haematoma within a 5 year period in our institution. The common feature in all was the anticogulation theraphy.

Methods: From January 2004 to December 2009, 25 patients (10 male, 15 female; median age 74 , range 40-89) in anticolagulant therapy with rectus sheath haematoma admitted to our emergency department presenting severe abdominal pain. For each patient serial clinical and laboratory data were collected (mean arterial pressure, pulse rate, pain, hemoglobin level, PT, INR and aPTT ) and linked to imaging study of CT (evaluating localization, extension and size of the hematoma). In all patients were administered fresh frozen plasma.

Results: 24 patients underwent urgent surgical treatment in view of the progressive haematoma (enlargement documented by $\mathrm{CT}$ ), haemodynamic compromission and worsening pain. Only one patient, a 74-year old women, died intraoperatively for C.I.D. due to the inability to control an active massive bleeding. In one case a 40 -year-old patient in hemodinamic stable conditions was treated conservatively with success. A total of 10 patients underwent radiological embolization to control arterial bleeding sources unsuccessfully.

Conclusions: This retrospective review indicates, in our opinion, that rectus sheath haematoma occurring in patients undergoing anticoagulation therapy should be managed surgically without delay. As our findings in this case series suggest, rectus haematoma -especially in older patient on oral anticoagulant medications -is not self limiting and can also have fatal evolution. In fact the clinical course of this entity is characterized by progressive enlargement of the haematoma size (as documented by serial CT scan in all patient), decrease of haemoglobin level and consequent hemodynamic instability. Nevertheless this finding can also suggest that earlier attempts with prompt surgical intervention could be associated with a better clinical prognosis by reducing the risk of inability to localize or control the bleeding vessels.

\section{8}

\section{Clipless Laparoscopic Cholecystectomy in 800 Consecutive Patients}

\author{
F. Bertone, G. Bottani, E. Gerosa, E. Bastaroli, A. Zanardi, \\ P. Petrosillo
}

Azienda Ospedaliera Pavia Ospedale Asilo Vittoria Mortara

Introduction: The laparoscopic technique has become the method of choice for cholecystectomy in the various phases of diseases of the gallbladder. Using the ultrasonic scalpel in all phases of the operation, allows bloodless dissection and a section of the elements without any clips or metal ligatures. We evaluated the use of ultrasonic scalpel in laparoscopic cholecystectomy performed in the last thirteen years.

Materials and Methods: We treated between May 1997 and May 2010, 800 patients gallstones in: 420 and 380 with acute cholecystitis with chronic cholecystitis intervention was conducted with ultrasonic scalpel (Ultracision ${ }^{\circledR}$ ). The technique is performed with isolation of separate elements of Calot's triangle before section- welding. The movement of blade in contact with the tissues causes the denaturation collagen molecules form a clot that closes the vessels and ducts.

Results: 800 patients operated on 3 cases $(0.4 \%)$ of biliary fistula with low flow, which resolved spontaneously in the third day, a case with endoscopic papillotomy.

Conclusion: The effect is purely mechanical, no electricity then provides security for the patient and operators. This avoids the placement of clips with all the advantages of absence foreign bodies, with a single instrument accomplishes the intervention without other pliersm. As a disposable instrument allows no risk of infection and saving on cleaning of instruments. The limited diffusion is related to the cost still high.

19

Robotic-Assisted Laparoscopic Insertion of
Gastric Electrical Stimulator (GES) Improves
Quality of Life in Patients with Gastroparesis

A. Rotundo, J. Gahir, C. Arhi, C.B. Tang, M. Harvey,

S. Kadirkamanathan

Department of Upper Gastrointestinal Surgery, Broomfield Hospital - Chelmsford, UK

Introduction: GES improves the quality of life in patients with drug refractory gastroparesis.

Material and Methods: Prospective study of 9 patients who underwent robotic-assisted GES insertion from September 2009 to March 2010 completing the SF-36 questionnaire before and after surgery. The following features of the SF-36 have been analyzed: general health, physical functioning, physical health, emotional problem, pain, energy and emotions. Surgical procedure: two electrodes are placed on the greater curve of the stomach, $1 \mathrm{~cm}$ apart and $10 \mathrm{~cm}$ proximal to the pylorus. The robot (da Vinci, INTUITIVE), allows for precise placement of the electrodes into 
the subserosal layer, confirmed by simultaneous gastroscopy. The electrodes are secured with interrupted intra-corporeal sutures and then connected to the IPG device which is placed in a subcutaneous pocket in the anterior abdominal wall.

Results: Median age was 37 years (range 24-47). Eight were female. There were 6 diabetics. The median duration of the whole procedure was 130 minutes (range 105 -218). Median length of stay was 5.5 days (range 1-45). 6 patients had medically-assisted nutrition before surgery and none after. The Median duration of nausea reduced from 18 hours per day (range 8-24) to 0 (range $0-10)$, Median episodes of vomiting reduced from 5 episodes per day (range 0-24) to 0 (range 0-6). There was improvement in SF-36 scoring with regard to general health, physical functioning, physical health, emotional problem and emotion but not to energy or pain.

Conclusion: Robotic-assisted laparoscopic insertion of GES improves symptoms as well as quality of life.

20

Magnetic Esophageal Sphincter for the Treatment of Gastro-Esophageal Reflux Disease: Results of a Prospective Clinical Trial

M. Nencioni, G. Saino, L. Laface, D. Bona, E. Asti, E. Abate, A. Sironi, S. Siboni, L. Bonavina

U.O. di Chirurgia Generale, IRCCS Policlinico San Donato, Università degli Studi di Milano

Background: We report our experience in GastroEsophageal Reflux Disease (GERD) surgical therapy with LINX device from March 2007 to April 2010. The aim of the study was to assess the efficacy of augmenting the esophageal sphincter barrier using a magnetic ring surgically placed at the gastro-esophageal junction.

Methods: Forty-nine patients with typical GERD symptoms responsive to PPI therapy and an increased esophageal acid exposure were enrolled in the study; 30 patients were enrolled for the feasibility clinical trial while 19 patients were selected for continuation of the clinical experience with the device. Patients with an abnormal esophageal motility were excluded. Preoperative and postoperative GERD typical symptoms were evaluated with Velanovich HRQL index score in both groups. Feasibility patients underwent esophageal $\mathrm{pH}$-study at one and two years postoperatively.

Results: Twenty-nine of the feasibility patients underwent one year post operative evaluation, 15 at two years. The second group of patients completed the 3 months follow-up. Mean esophageal acid exposure $(\mathrm{pH}<4)$ significantly decreased from $10.5 \%$ $(\mathrm{N}=49)$ over total time of study to $3.5 \%(\mathrm{~N}=29)$ at one year and $2.6 \%(\mathrm{~N}=15)$ at two years $(\mathrm{p}<0.001)$. Mean GERD-HRQL score passed from 21.4 at baseline $(\mathrm{N}=49)$ to 3.4 at 3 months $(\mathrm{N}=48)$, 3.1 at one year $(\mathrm{N}=29)$ and 2.1 at two years $(\mathrm{N}=15)$. Mild and transient dysphagia were reported in 27 patients (55.1\%). No migrations or erosions of the device occurred.
Conclusions: Laparoscopic implant of the LINX device is a safe and reproducible procedure that allows to reduce esophageal acid exposure and improve GERD typical symptoms in selected patients.

21

\section{The Tailored Surgery in Incisional Hernia Repair}

\author{
M. Canziani, S. Agrusti, V. Bertocchi, M. Cavalli, \\ G. Campanelli
}

University of Insubria, Varese, Italy, Dept. of Surgical Sciences Unit of General Surgery, Day and Week Surgery (Chief: Prof. G. Campanelli), Multimedica Hospital, Castellanza (va), Italy

Introduction: Incisional hernias are a common complication in abdominal surgery. The best technique to correct incisional hernias is by using synthetic meshes; in case of loss of substance or direct contact of the mesh with bowel, composite meshes must be used; recently biological meshes are reaching agreement in incisional hernia repair.

Materials and Methods: Between 2002 and 2009, 243 pts underwent open surgical repair of incisional hernias.

Direct suture was performed in 8 pts; mesh repair, using syntetic mesh fixed with suture was performed in 100 pts and with fibrin glue in 10 pts. Component separation was performed in one patient, composite meshes were used in 5 pts and biological meshes in 4 pts. In case of loss of substance, a double mesh was used in 68 pts and a composite mesh in 7 pts; in 40 pts with recurrent incisional hernia, a PP mesh was used.

Results: The average hospitalization period was 4 days; postoperative complications were wound infection in $7 \mathrm{pts}$, hematoma in 9 pts, intestinal occlusion in 6 pts, fistula in 2 pts, chronic infection in 2 pts and stiff abdomen in 3 pts. Seroma was observed in 12 pts, chronic pain occurred in 3 pts and recurrence in 3 pts.

Conclusion: Superiority of mesh repair versus direct suture is commonly accepted, but there is no evidence of differences by using different meshes. In our experience different hernias require different techniques and different meshes, so it's very important to perform a procedure "tailored" on the patient. 
22

\section{Endoluminal Vacuum Therapy for Anastomotic Leaks After Rectal Surgery: Analysis of $\mathbf{3}$ Paradigmatic Cases}

\author{
A. Arezzo, M.E. Allaix, A. Miegge, A. Garbarini, M. Morino \\ Digestive, Colorectal and Minimal Invasive Surgery, \\ University of Torino - Corso Dogliotti, 14 - 10126 Torino, \\ Italy
}

Introduction: Anastomotic leakage after rectal surgery is a serious complication. We describe 3 cases treated with EndoSPONGE.

Material and Methods: A 62-year-old woman complaining local discomfort and fever 7 days after a Delorme procedure, a 73-year-old man with a persistent anastomotic leakage 3 months after TME and ileostomy following chemoradiotherapy, and a 69-year-old man with fever and a wide anastomotic leak 4 weeks after TME and ileostomy were treated.

Results: In the first case, an anterior anastomotic leak, associated to an abscess, communicated with the vagina. The EndoSPONGE was inserted, without clinical improvement after 6 days. A colostomy was performed. The Endo-SPONGE was replaced: at 21 days the anastomosis was completely covered by mucosa.

In the second case, a 3-mm anastomotic leak underwent balloon dilatation, revealing a chronic paranastomotic abscess; after Endo-SPONGE insertion, granulation tissue progressively filled the cavity, without a complete closure of the fistula orifice. Since the patient was asymptomatic, the ileostomy was closed.

In the third case, an Endo-SPONGE was inserted. After 96 hours without clinical improvement, a CT scan showed a large amount of free air, for which APR was performed.

Conclusion: With adequate selection of patients based on leak, local conditions and systemic disease Endo-SPONGE revealed impressive effectiveness.

\section{3}

\section{Pancreas: A Systematic Review About an Uncommon Site of Metastasis}

S.A. Bonapasta ${ }^{1}$, M. Gregori ${ }^{1}$, A. Menghi², R. Lanza²

S. Praticò ${ }^{3}$, M. Miccini ${ }^{1}$, A. Tocchi ${ }^{1}$

${ }^{1}$ Department of Surgery Pietro Valdoni, University of Rome Sapienza, ${ }^{2}$ Department of Clinical Oncology, University of Rome Sapienza, ${ }^{3}$ Department of Statistical Sciences, University of Rome Sapienza

Introduction: Metastasis to the pancreas from malignant tumors is a rare event. Pancreas may be involved from lymphatic or haematogenous dissemination. The metastatic disease can be confined to the pancreas (isolated) or can involve other sites (not isolated). The pancreatic secondary can present as a single lesion (solitary) or can consist of multiple lesions.

Materials and Methods: A detailed review of case reports, retrospective and prospective studies has been conducted. Sites of primary tumor, interval between primary tumor and pancreatic metastasis, pancreatic site, size of the lesion, tumoral markers level, radiologic description, pre-operative diagnosis, survival have been recorded when available. Variables were compared using Pearson's $\chi^{2}$ and T-Student tests. Survival was analysed using Kaplan-Meier and Mantel-Cox methods.

Results: We considered 162 articles from 1974 to 2009, including 670 patients with 27 different sites of primary. Solitary isolated metastases are often misdiagnosed as primary neoplasms of pancreas. Metastases from renal cell cancer (RCC) are mostly correctly diagnosed, because they are more common and present as highly vascular lesions. Metastasectomy in patients with RCC can prolong survival, while other metastases could be more susceptible to chemotherapy.

Conclusions: Metastatic disease to the pancreas must always be considered in patients with pancreatic lesion and any history of a preceding malignancy. Accurate diagnosis is essential for planning management and predicting prognosis. The treatment should be individualized and influenced by the site and the histologic type of primary tumor, the clinical demands, the kind of necessary resection and the physical and mental status of the patient.

\section{4 \\ Urgency: Is Laparoscopy an Adeguate Approach? Our Experiece

\author{
D. Apa, B.C. Brassetti, E. Puce, G. Senni, F. Atella, \\ M. Lombardi \\ General Surgery CTO A. Alesini -Rome
}

Methods: From 1991 to 2009 a total of 5600 patients underwent emergent or urgent surgery. Among them 727 (388 women and 339 men, mean age 45 years, range 9-98) were operated laparoscopically: 280 acute cholecystitis (38.5\%), 266 appendicitis (36.5\%), 60 pelvic inflammatory disease (8.3\%), 41 bowel obstruction $(5.6 \%), 36$ perforated peptic ulcer (5\%), 14 peritonitis of different aetiology (2\%),13 perforated diverticulitis (1.8\%), 10 traumatic lesions (1.4\%), 3 spontaneous rupture of liver tumours $(0.4 \%), 2$ colon cancer perforation $(0.3 \%), 2$ heamoperitoneum $(0.3 \%)$. The primary aim of laparoscopy in the trauma will be diagnostic with accuracy rate of $100 \%$.

Results: The conversion rate was about 7\% (52 patients) and was mainly observed in heamoperitoneum (82\%), faecal peritonitis $(54 \%)$ or intestinal occlusion (34\%). The conversion was often due to the presence of dense intra-abdominal adhesions or to main bowel distension. Laparoscopic lavage-drainage and diversion was performed in 3 cases of diverticulitis Hinchey III-IIB; 3 patients with faecal peritonitis underwent a laparoscopic Hartmann's procedure. Morbidity and mortality rate were $5.3 \%$ and $0.7 \%$ respectively. The mean operative time was 90 minutes and the mean hospital stay was 7 days (range 2-10).

Conclusions: Laroscopic approach is firmly established superior to open surgery for many acute abdominal diseases. In stable patients with acute pain non-invasive diagnostics should be fully exhausted before considering explorative surgery. In trauma has a role in haemodynamically stable patients, specially in penetrating thoraco-abdominal injuries for evaluation integrity of 
diaphragm. Primary laparoscopic lavage for complicated diverticulitis may be a promising alternative to more radical surgery in selected patients with a lower incidence of wound infections.

\section{5}

The Colon Flap Extension Malone Antegrade Colonic Enema (MACE) in the Treatment of Patients with Severe Stypsis Due to Traumatic Spinal Cord Injuries: Case Report

F. Papini, R. Caronna, M. Schiratti, G. Prezioso, G. Fanello, M. Benedetti, R.L. Meniconi, F. Farelli, G. Dinatale,

G. Russillo, C. Benucci, L. Argentino, P. Chirletti

"SAPIENZA" Università di Roma - Policlinico Umberto I, Dipartimento di Chirurgia "F. Durante" - U.O.C. Chirurgia

Generale N, Scuola di Specializzazione in Chirurgia

Generale V (indirizzo in Chirurgia d'Urgenza)

Direttore Prof. P. Chirletti

Introduction: Defecation in patiens with spastic-discynetic bowel disfunctions or post-traumatic complete spinal cord injuries can be difficult and is associated to abdominal pain, abdominl distension and respiraory distress due to the diafragmatic distension.

Materials and Methods: A 40 yrs old man was admitted for chronic intestinal subocclusion resistent to therapy. He had a post-traumatic spinal cord injuryand had already been operated of appendicectomy and colonic suture for a laceration of the right colon. We scheduled a Malone antegrade continence enema (MACE), consisting in an anterograd percutaneus irrigation of the colon by inserting a catheter through a modified appendicostomy. This was realized with a colon flap extension as an alternative technique for those patients that had already been treated with appendicectomy or right emicolectomy.

Results: After the operation the patient could evacuate after 40 minutes from the anterograde enema through the appendicostomy. This improved the abdominal distension, the respiratory distress, allowed him to program regular evacuations every 2 days and avoided him a terminal colostomy.

Conclusions: For the low rate of post operative complications (5\%), the absence of intestinal anastomoses and the efficacy of the continent valve mechanism (98\%), the colon flap extension MACE is considered to be a good therapeutic option for these patiens.

\section{6}

\section{Mammoglobin Expression is Related to Favorable Prognosis in Breast Cancer}

\author{
F. Bettarini, G. Corso, S. Caruso, M. Di Martino, G. Flauti, \\ D. Marrelli, F. Mariani, F. Roviello, E. Pinto, A. Neri
}

Surgical Oncology Unit, Department of Human pathology and Oncology, University of Siena

Background: The human mammoglobin (h-MAM) encodes an epithelial secretory protein of the uteroglobin family. h-MAM expression is restricted to normal breast epithelium and it is frequently up-regulated in breast cancer. h-MAM has been studied as a marker of metastases in blood, lymph nodes and bone marrow, but a significant proportion of breast cancers does not express h-MAM.

Materials and Methods: Expression of h-MAM in breast cancers has been studied in 70 samples of fresh frozen tumor tissues from breast cancer patients. Clinical and histopathological parameters were recorded and follow-up data were collected at our outpatient clinic. To assess h-MAM gene expression, quantitative real-time PCR was performed, using $\beta$-2-microglobulin as housekeeping gene and calculating differences in gene expression after gene amplification.

Results: h-MAM resulted over-expressed in 21 cases (30\%), and was significantly related to age less than 60 y.o., premenopausal status, grade 1 tumors and estrogen receptors (Er) expression. At a median follow-up of 130 months, 30 relapses of disease were recorded and 23 women died of breast cancer. At univariate analysis, h-MAM expression resulted significantly related to better DFS and OS, together with smaller tumor size, absence of axillary nodal metastases and presence of Er receptors. At multivariate analysis, tumor size and nodal status resulted independent prognostic factors together with Er expression for DFS and lymphovascular invasion for OS respectively; h-MAM did not result an independent prognosticator.

Conclusions: h-MAM was over-expressed in about one third of breast cancers and was related to biological parameters of better prognosis. Indeed, h-MAM was significantly related to better DFS and OS, but did not result an independent factor, probably because its positive prognostic impact is related to such favourable parameters.

\section{7 \\ Preventive Laparostomy in an Emergency Surgery and Trauma Service: A Three Years Experience}

\author{
S. Boati, S. Cimbanassi, O. Chiara \\ SS Trauma Team -Ospedale Niguarda Ca' Granda Milano
}

Introduction: Open abdomen $(\mathrm{OA})$ is a surgical option that is increasing in trauma and emergency surgery. In our hospital we largely use OA as a preventive technique of increased intrabdomi- 
nal pressure (IAP). This is a preliminary analysis of our experience in trauma and inflammation, during a three years period.

Methods: A temporary abdominal closure (TAC) was performed if IAP was $>12 \mathrm{mmHg}$. Indications were: severe acute pancreatitis (2), bowel infarction (3), abdominal compartment syndrome (1), peritonitis (17), hemoperitoneum (12). A packing with a temporary skin closure was performed in six patients and a home-made vacuum-assisted closure in the others.

Results: Between january 2007 and december 2009 thirtyfive patients underwent TAC. In survived patients early abdominal closure (1- 4 days, median 1) was obtained in traumatic bowel injuries, hemoperitoneum and bowel infarction, while in peritonitis and pancreatitis closure was performed later (1-10 days, median 4). Non survived patients were 11, 6 peritonitis, 3 hemoperitoneum, 1 pancreatitis and 1 bowel infarction, all with prolonged periods of OA ( 1-48 days, median 5). Mortality seemed related to severity of disease causing high values of IAP which prevenedt closure. Complications were: wound infections (3), intestinal fistula (5) and abdominal abscesses (2).

Conclusions: Current evidence supports the use of TAC for prevention of abdominal compartment syndrome because a normal IAP ameliorates physiology in critical illness. In this sense, the need of a longer $\mathrm{OA}$ is a consequence of continuously increased IAP which is a sign of not resolved underlying illness.

\section{8 \\ Surgical Management for Metastatic Testicular Germ Cell Cancer: Our Experience \\ J. Brandolini, M. Salvi, G. Grani, A. Chapelier, A. Lucchi, P. Berti, F. Vandi, G. Garulli \\ U.O. Chirurgia Generale-Toracica, Ospedale di Riccione, Rimini}

Introduction: Testicular tumors are rare diseases affecting young males, but their incidence is increasing. Advanced disease is curable in $80 \%$ of the cases. The primary site of metastases is the retroperitoneal lymph nodes. Secondary metastatic sites are the lungs and mediastinal lymph nodes. Resection of residual postchemotherapy pulmonary masses in patients with non-seminomatous germ cell tumours gives therapeutic benefit. We analyzed the outcome and prognostic factors after surgery.

Materials and Methods: Authors presents 18 patients who were surgically treated for metastases in germ cell testicular cancer. All patients were allocated for surgical treatment after undergoing cisplatin-based chemotherapy.

Results: In this group of 19 patients, we have chosen a laparotomic approach for all the cases.

For 5 cases we have done a sternotomy and for 3 cases a postero-lateral thoracotomy.

Five patients have been undergone to a neck dissection and other five to a sub-carenal dissection. Overall, 14 of 18 patients had necrosis, 3 had carcinoma, and 1 had unresectable residual masses above brachial plexus, identified in permanent section analysis. There wasn't any operation mortality. The medium stay in hospital was of 14,4 days. The total survival for 5 years was of the $92 \%$, while the free interval from the illness to 5 years was of the $87 \%$.

Conclusions: We conclude that pulmonary resection in metastatic non-seminomatous germ cell tumors is a safe and effective treatment modality. High serous levels of beta-Hcg, an adverse histology and an incomplete surgical resection represent the greatest prognosis factors associated to a negative outcome.

\section{9 \\ Acute Pancreatitis Caused by Pancreatic Metastasis of Small Cell Lung Carcinoma \\ A. Coppola, G.T. Capolupo, F. Longo, I. Cipollone, \\ Department of General Surgery, University Campus Bio/ Medico school of Medicine, Rome}

Introduction: Primary Small Cell Lung Carcinoma frequently metastasizes to distant organ. The pancreas is a relatively infrequent site of metastasis.

Materials and Method: A 54-yr-old man was admitted to our department, with diagnosis of acute pancreatitis with the typical epigastric pain, nausea, vomiting and general weakness and malaise. Fever and jaundice were absent. Abdominal ultrasound showed a mass in the pancreatic body, confirmed by an abdomen CT scan suggestive for primitive pancreatic tumor.

Extensive staging workup of the pancreatic neoplasm revealed a bulky mass in the mediastinum, with carinal adenopathies. EUS confirmed the mediastinic and pancreatic mass. The patient underwent during EUS procedure FNAB of the pancreas and biopsy of the mediastinic mass. The immunohistochimic essay demonstrated a positive match for pancitocheratins and chromogranin. Results These positive test suggested the diagnosis of SCLC with a pancreatic metastasis. The patient was submitted to systemic chemotherapy with CBCDA+VP16 and CBCDA+Taxolo regimen.

Conclusion: Patients with SCLC remains asymptomatic until the disease therefore is at an advanced stage and often it happens that diagnosis comes after unusual symptoms not related to the primary site of disease. There is disagreement in the literature about the frequency of the primary sites that give rise to metastases in the pancreas. In most studies, lung cancer is the most frequent type of malignancy causing pancreatic metastases, especially SCLC.

The correct diagnostic approach includes CT scan, EUS and biopsy of the lesions to permit a right diagnosis and the possibility of a well timed therapy.

Eur Surg Res 2010;45:158-307 
30

\section{Hyalomatrix Employ After Surgical Removal of an Eccrine Scalp Spiroadenocarcinoma}

\author{
A. Dondas ${ }^{\circ *}$, A. Ascari Raccagni*, F. Tauceri\# ${ }^{*}$ S. Asioli ${ }^{\omega}$, \\ M. G. Righini*, G. Trevisan ${ }^{\circ}$ \\ 'Dermatological Clinic, University of Trieste, "Department \\ of Dermatology, "G.B. Morgagni - L.Pierantoni General \\ Hospital”, Forlì, 'Department of Pathology, "G.B. Morgagni \\ - L.Pierantoni General Hospital", Forlì, "Department of \\ Advanced Oncological Surgery and Therapy, "G.B. \\ Morgagni - L. Pierantoni General Hospital", Forlì
}

Introduction: A-45-year old patient from Nigeria came under our observation, he was in good health conditions but with spread metastasis due to a cutaneous neo-formation located on the occipital site.

An eccrine scalp spiroadenocarcinoma was histologically diagnosed, a sweat glandulae tumor, rare, aggressive and inclined to metastasis.

The cutaneous lesion rapidly progressed becoming large, exophytic, ulcered, bleedy and foul-smelling.

The patient had already undergone a cycle of polichemotherapy.

Method: Aware of the palliative value of the surgery we have tried to treat the cutaneous neoplasia in the most thorough and simple way with the least aggressive surgery and an exclusively outpatient post-surgery.

The tumor has been thoroughly excised under total anesthesia by removing the pericranium.

The loss of substance has been partially sutured reducing its size and a plaque of Hyalomatrix has been applied after having shaped it and sutured on the edges.

Materials: Hyalomatrix is a dermis replacement made with HYAFF a hyaluronic acid ester which supplies a fibroblast tridimensional structure for the rebuilding of the skin with a semiwaterproof silicon film on the outer side.

The Hyalomatrix has been left on site for 3-4 weeks with weekly follow-ups.

Results: In our patient we have observed after 3 weeks that the defect was completely covered with the new dermis which could have been later repaired with a thin cutaneous graft or left to heal for further considerations.

Conclusions: Hyalomatrix can be taken into consideration in the reconstruction of scalp large substance losses even when the pericranium has been removed.

\section{1 \\ Right-Sided Diverticulitis in Urgency: 3 Cases-Report}

\author{
F. Tauceri, D. Dipietrantonio, G. Solfrini, M. Framarini, \\ F. D'Acapito, A. Vagliasindi, D. Cavaliere, G.M. Verdecchia
}

Surgical Oncology, Morgagni-pierantoni Hospital, Forli', Italy

Introduction: Diverticulitis of the ascending colon is very uncommon in the Occident: only 1 case for every 300 appendicites in Western countries, while in Asia is more frequent the right localization, due to genetic factors. The peak age of symptoms is 40-45 years, with a sex ratio of 1:1. The presentation symptoms are similar to appendicitis. Other differential diagnoses are: right colonic cancer, Meckel diverticulitis, inflammatory diseases, hepatic abscess. The best tool for the diagnosis is CT-scan. In noncomplicated cases, a conservative antibiotics therapy is possible. Surgical treatments are: diverticulectomy with appendicectomy; right colectomy; ileo-caecal resection. Actually a real surgical therapeutic strategy is not still validated. Where the disease is more frequent, conservative therapy is the rule.

Material and Methods: We report 3 cases ( 1 M, 2 F; mean age: 61) of right diverticulitis operated in urgency. The main symptom was abdominal pain in all the cases; in 2 cases the echography was negative, while in all the cases the diagnosis was made with CT-scan (a solid mass in the right abdomen).

Results: In all the cases the therapy was surgical: right colectomy; tiflectomy with linear stapler; ileo-caecal resection. No complications appeared. The mean hospitalization was 8 days. The histological setting confirmed the diverticulitis presence. All patients are free of pain.

Conclusions: It's difficult to treat right diverticulitis with conservative therapy, because it's frequent in differential diagnosis with appendicitis. The CT-scan is the best tool for the diagnosis and the surgical treatment, even if enlarged, is feasible without complications.

\section{2 \\ Pancreatic Metastasis from Uterine Leiomyosarcoma: A Case-Report}

F. Tauceri, G. Solfrini, F. D'Acapito, D. Dipietrantonio, M. Framarini, A. Vagliasindi, D. Cavaliere, G.m. Verdecchia

Surgical Oncology, Morgagni-pierantoni Hospital, Forli', Italy

Introduction: Leiomyosarcoma uteri is a highly malignant neoplasm, $1.3 \%$ of all uterine cancers. Report incidence is 0.3 0.4 per 100000 women per year. It appears similar to leiomyoma. Frequently asymptomatic, suspicion should be high in rapid uterine increase after menopause, severe abdominal pain, vaginal bleeding. It metastases to lung and liver, while rarely to small bowel, heart, brain and thyroid, by direct extention, implantation, haematologic or lymphatic route. Primary therapy entails com- 
plete surgical resection: total abdominal hysterectomy, bilateral salpingo-ophorectomy. The lymph nodes evaluation remains controversial. Adjuvant treatments include gemcitabine-docetaxel or doxorubicin with or without ifosfamide. The majority of patients recur in 2 years. CASE PRESENTATION:A 64-year-old was referred with abdominal pain and diarrhea. On examination, she appeared with a pelvic mass. Rectal examination (while pelvic one was negative) detected a palpable mass on the anterior rectum. A thorax-abdomen TC saw a $12 \mathrm{~cm}$ diameter uterine mass, pressing the colon, bladder and iliac vein and a "target-image" in the pancreatic body. A TC-PET confirmed the uterine and pancreatic neoplastic uptake. At laparotomy, an enlarged $25 \mathrm{~cm}$-diameter mass from the uterus was resected, through a total abdominal hysterectomy, bilateral salpingo-ophorectomy, segmental colonic and bladder resection, bilateral iliac-obturator lymphadenectomy. On the suspected pancreatic metastasis, a needle-biopsy was made. The result of pathology was a high grade poorly differentiated leiomyosarcoma, in fibroleiomyomatosus uterus, infiltranting bladder peritoneum, sigma and pancreas. All the limph nodes were negative.

Conclusions: We present this unusual case of pancreatic metastasis from uterine leiomyosarcoma.

33

\section{Evaluation of Stress Response in Laparoscopic Liver Surgery: A Prospective Study of Inflammatory Profile, Coagulation Homeostasis and Clinical Outcome}

\author{
F. Cipriani, F. Ratti, E. Guzzetti, M. Catena, R. Finazzi, \\ L. Aldrighetti \\ IRCCS San Raffaele Hospital - General and Specialistic \\ Surgery Department - UOC Hepatobiliary Surgery - Milan \\ - Italy
}

Introduction: It is known that an inflammatory response and hypercoagulability state develop after surgery. Laparoscopy for liver resections is still in its infancy. The aim is a case-matched analysis about the clinical outcome, inflammatory profile and coagulation homeostasis of laparoscopic liver resections.

Material and Methods: 24 liver resections were assigned to the laparoscopic approach and the further 24 to the open procedure.

Results: Postoperative plasma levels of AST, ALT, WBC, CRP, IL-6, PT and D-dimer showed a lower rise in laparoscopic group. Intraoperative blood losses, blood transfusions rate, analgesic therapy amount, time of mobilization, fasting duration, time of first flatus and overall morbidity resulted lower in laparoscopic group. Decrease of platelets, antithrombin III and fibrinogen levels was lower in laparoscopic group. Postoperative levels of bilirubin and TNF- $\alpha$, operating time, tumor exposure at the transection surface and minimal surgical margins resulted comparable between the two groups.

Conclusion: The laparoscopic approach for liver resections seems to result in improved clinical outcome, lower stress response and lower coagulation alterations.
34

\section{Minimally-Invasive Approach in Liver Surgery: Laparoscopic Left Lateral Sectoriectomy}

F. Cipriani, L. Aldrighetti, M. Catena, F. Ratti, G. Ferla

Department of Surgery-Liver Unit, Scientific Institute H San Raffaele, Vita-Salute San Raffaele University, Milan, Italy

Introduction: Laparoscopy is gaining acceptance as a safe procedure for resection of liver neoplasm, but its feasibility and safety remains to be demonstrated.

Material and Methods: Single laparoscopic left lateral sectoriectomy performed at our institution (Department of SurgeryLiver Unit, Scientific Institute H San Raffaele, Vita-Salute San Raffaele University, Milan, Italy).

Results: Laparoscopic liver left lateral sectoriectomy is safe and feasible.

Conclusion: In the next future laparoscopic approach for liver resections may become the gold-standard for selected patients.

\section{5 \\ VAC (Vacuum Assisted Closure) Treatment in Fournier's Gangrene. Preliminary Experience}

V. Cozza, F. Passafiume, L. Valesini, M. Assenza

Introduction: Fournier's gangrene (FG)is a rapidly developing necrotizing fasciitis that originates in genital and perineal region. The mortality rate is high and requires prompt diagnosis, antibiotic treatment and extensive necrosectomy with derivative colostomy. Vacuum Assisted Closure (VAC) is a wound care system of paramount importance in the treatment of complex wounds, including the perineal region.

Materials and Methods: We evaluated 4 cases of FG (males, mean age: $62.5 \mathrm{yrs}$ ) of the last 2 years. All patients were diabetics. We used intravenous antibiotic treatment and early surgical debridement with colostomy, followed by immediate positioning of a VAC device (NP 125-200 mmHg). The dressing changes were done every 3-4 days. No hyperbaric oxygen therapy was given. Microbiological etiology was assessed by multiple cultures to tailor the antimicrobial treatment.

Results: The VAC therapy reduces the number of dressings and the hospital length of stay (LOS), in agreement with the literature; in one of the four cases a secondary a reconstructive surgical intervention was possible. The colostomy was reversed in all patients within 3 months.

Conclusions: Negative pressure is a time saving device, reducing days of hospitalization, patient's discomfort and number of medications. The possibility of a early reconstructive surgery improves quality of life. 
36

\section{Surgical Treatment in Case of Postoperative Complication Following Longo Procedure: Our Experience}

\author{
D. Crocetti, F. Guerra, V. Cozza, E. Sacco, M. Assenza, \\ F. La torre \\ Specialità: Chirurgia Generale
}

Descrizione Abstract: This study analyzes 202 patients, (130 women, 72 men; age $25-80$, average 50,8), that have been subjected to coloproctological visit from January 2003 to Dec 2009. Each patient showed symptoms correlated to stapled haemorrhoidectomy. A corrective surgical treatment was proposed to the patients we selected ( 85 patients: 48 women, 37 men) with pain, anal stenosis and/or substenosis. Each patient was subjected to a careful anamnesis, inspection, rectal exploration, anoscopy, anorectal manometry and defecografy. The correction consists into a deep $180^{\circ}$ wide lateral section, a breaking down the scarring and, if needed taking away some residual stitches. Post-operative course was of 2 days, with 7, 21,60 days. From the 85 selected patients we operated 48 cases: we have had a full remission of the pain in $78 \%$ (38 patients), the remaining 22\% (10 patients) having had to be subjected to out-patient remotion of the stitches. The procedure of circular stapler prolassectomy should have to be reserved just to the third degree haemorrhoid disease with mucosal prolapse, performing the anasthomosis beyond the pectinate line of about $2 \mathrm{~cm}$, according to the brand patent. We observed patients which have improperly undergone stapled procedures with moderate to severe complications. We want to stress that Milligan Morgan procedure is the gold standard treatment for the haemorrhoid up today.

\section{7}

Liver Resection for Colo-rectal Metastasis in Elderly Patients: A Case-control Study

\author{
G. D'Amico, F. Di Benedetto, R. Montalti, N. De Ruvo, \\ N. Cautero, G.P. Guerrini, R. Ballarin, M. Spaggiari, \\ N. Smerieri, G. Tarantino, V. Serra, M. Marino, \\ R.M. lemmolo, A. Mimmo, G.E. Gerunda
}

Liver and Multivisceral Transplant Center, University of Modena and Reggio Emilia Modena, Italy

Introduction: As the mean life expectancy rises, the incidence of patients 70 years of age or older who present with colorectal liver metastases (CLM) continues to increase. This study was conducted to investigate the safety as well as short- and longterm outcomes of liver resection for CLM in elderly patients.

Materials and Methods: Patients were grouped according to age: 70 years of age or older (elderly group) and younger than 70 years old (younger group). We performed a case-control analysis with a 1:1 ratio, comparing the elderly group $(n=32)$ with a younger counterpart $(n=32)$. Data were analyzed according to pre-, peri- and post-operative results, as well as long-term survival.

Results: Major resections (> 3 Couinaud's segments) were performed in $59.4 \%$ of elderly group and $66.5 \%$ of younger group. In this series, elderly patients were comparable to the younger counterpart in terms of operative duration, transfusion rate, postoperative hospital stay and complications. The morbidity rate was $28.1 \%$ in the of elderly group and in $34.4 \%$ in the younger group $(\mathrm{p}=1)$. The 30 -day and 60 -day mortality rates, the overall and disease-free survival were comparable between the two groups.

Conclusion: Advanced chronological age cannot be considered as a medical contraindication for elderly patients underwent hepatic resection for CLM. This study provides further evidence in term of resection for CLM in the elderly population: surgical treatment can be performed safety with low mortality and acceptable morbidity rates, offering a long-time survival advantage to many of these patients.

\section{8 \\ Rectal Cancer and Duodenal Cancer in a Patient with Common Variable Immunodeficiency}

S. Manfredelli, A. Angelici, M. Bezzi, C. Dezzi

Dipartimento di Chirurgia "Francesco Durante"- Università degli Studi di Roma Sapienza

Background: Common variable immunodeficiency (CVID) is a primary immunodeficiency characterized by a low level of antibodies and may become evident during infancy, childhood, puberty, or even later into adulthood. In CVID there is an increased risk of developing some cancers, expecially lymphoma (from $23 \%$ to $100 \%$ ) and gastric carcinoma (50\%).

Case Presentation: Authors report the case of L.P. 37 years old patient with CVID. In October 2008 patient were treated for a rectal cancer with anterior resection of rectum with T-T anastomosis (pT2pN0pMx G3 St.I) followed by adjuvant chemotherapy. Follow-up doesn't show neoplasia disease. In October 2009 patient were treated for duodenal cancer by duodenal-jejunal resection with T-T duodenal-jejunal anastomosis (pT3pN1pMx G2 St.III). Actually patient is in follow-up and has a good quality of life.

Conclusions: We report a case of rectal and duodenal cancer in a 37 years old man with CVID; current Literature doesn't report a similar case as we expose. 


\section{9}

\section{Case Report: Laparoscopic Cholecystectomy in a Patient with Situs Inversus Totalis}

\author{
C. Dezzi, V. Urbano, A. Forte, M. Bezzi, S. Manfredelli
}

Dipartimento di Chirurgia "Francesco Durante"- Università degli Studi di Roma Sapienza

Background: Situs inversus totalis is inherited as an autosomal recessive trait and occurs in approximately 1 per 10 to 20.000 live births. It's definite as the transposition of both the thoracic and abdominal viscera to the opposite side of the body.

Case Presentation: D.D. was a 42 y old man who experienced 6 months prior of his presentation intermittent nausea,intermittent epigastric pain that radiated laterally to both sides of upper abdomen,shoulder pain and bloating after some meals. He received an abdominal US that showed the presence of gallstones without biliary tree distention, with gallbladder wall thickening. Patient underwent laparoscopic cholecystectomy. The operation was carried out in the usual manner with the trocars placed in locations on the left side of the abdomen as mirror images to their usual location on the right side. On laparoscopic examination the intra-abdominal anatomy was the mirror image of the normal view. Dissection of the triangle Calot and application of the clip and gallbladder dissection was performed by operator's left hand through the subxiphoid trocar.

Conclusions: Some authors suggest that laparoscopic cholecystectomy in patients with left-sided gallbladder is preferred to be performed by left handed surgeons due to better ergonomy; we suggest that surgeon should be right and left handed with experience in laparoscopy and hepatobiliary surgery.

\section{0}

\section{Gallbladder's Carcinoid: A Case Report and Review of Literature}

\section{Dezzi, S. Simonelli, M. Bezzi, V. Urbano, S. Manfredelli}

Dipartimento di Chirurgia "Francesco Durante"- Università degli Studi di Roma Sapienza

Background: Carcinoids are neuroendocrine neoplasms frequently localized in gastrointestinal tract $(73,7 \%)$. Endocrine tumors originated from gallbladder are very rare $(0,2 \%)$.

Clinical behaviour of this neoplasm depends on tumor size, local invasion and presence of metastatic spread: cases reported of poor prognosis after surgery are in patients with extended disease and liver metastasis.

Case Presentation: Patient woman of 78 years old with symptomatic gallstones, with abdominal pain located in the epigastrium radiating to right and left side, with vomiting bile and diarrhea. Patient were treated with laparothomic cholecystectomy for patient's choice without complications. To the anatomo-pathological examination the material envoy resulted constituted by colecisti of the length of $\mathrm{cm} \mathrm{8,7,} \mathrm{container} \mathrm{a} \mathrm{calculation} \mathrm{of} 12 \mathrm{~mm}$. In correspondence of the fund of the organ it noticed him below the mucous one the presence of a nodule of grey-yellowish complexion, of hard-elastic consistence, of the maximum diameter of $1 \mathrm{~cm}$.

The histological picture deposed for a diagnosis of gallbladder's carcinoid and the remainder wall of the gallbladder it was center of chronic cholecystitis.

Later around 2 weeks from the resignation the patient has been submitted total to TC body, that has given negative result for the search of possible metastasis.

To everything today, after 12 years from the surgical intervention, the patient is alive and free from illness.

Conclusions: The autors, starting from the case of carcinoid of the gallbladder they observed in a 78 year-old woman undergone cholecystectomy because of symptomatic gallstones, review the literature about pathologic aspects, symptoms and prognosis of this tumor.

\section{1 \\ Management of Obstructed Left Colon Carcinoma: Defining Role of Self -Expandable Metal Stents
B. Di Venere ${ }^{1}$, B. D'Ascoli ${ }^{2}$, A. G. Nasti ${ }^{1}$, G. De Maio², F. Bucci ${ }^{1}$
1 U.O. Complessa Chirurgia Generale- Direttore Dott. F. Bucci, ${ }^{2}$ U.O. Complessa di Gastroenterologia ed Endoscopia Digestiva - - Direttore Dott. G. De Maio, Ospedale Madonna delle Grazie - Matera

Introduction: Self-expanding metallic stents have found increasing use in the palliation of malignant left sided large-bowel obstruction or as a 'bridge to surgery' to facilitate a planned operative procedure that carries less morbidity and mortality compared to emergency surgery. In this study we report our experience, to evaluate the effectiveness and safety of a self expanding metal stent (SEMS), in the treatment of left-sided malignant colorectal obstruction.

Material and Methods: From June 2007 to January 2010, 32 consecutive patients with malignant colorectal obstruction located in the left colon underwent the positioning of SEMS.

Main outcome measures were the success rate in endoluminal stent placement and the efficacy in decompressing the obstruction avoiding colostomy.

Results: Successful stent placement was obtained in 30/32 patients (94\%). Among the treated patient the SEMS positioning was the definitive palliative treatment in 6 cases (18\%): patients aged, with advanced metastatic disease and in critical conditions. In two of this patients we observed a late complication a colonic perforation at more than 90 days from stent positioning, requiring emergency surgical treatment. In the others 24 cases the SEMS positioning was a bridge to elective surgery meanly at 12 days after stent placement.

Conclusion: In our experience SEMS placement is safe, effective and should be considered as initial non operative management in all patients seen with malignant left sided large bowel obstruction in absence of peritonitis. Other main outcome was the 
reduction of morbidity and mortality in surgery for colonic malignancy in this group of patients.

\section{2 \\ Intracellular Cytokines as Markers of Inflammation and Immune Response for Selecting Patients with Carotid Atherosclerosis to Undergo Endarterectomy}

G. Sciarra, G. Lacroce, M.C. Tesori, A. Metere, L. Marcuccio, E. Mastroluca, A. Esposito, A.R. Forcione

Department of Surgical Sciences, University “La Sapienza", Rome, Italy

Introduction: The process of atherogenesis is considered a chronic immune-mediated inflammatory disease: inflammation is a key factor in the process of plaque destabilization, and rupture, thrombosis. The main objective of our research is: identify immunological markers of vascular damage in peripheral blood of patients with carotid atherosclerosis; identify and characterize autoantigens, target of autoimmune responses in carotid atherosclerosis.

In the present study the AA. assessed: the possible role of intracellular pro- and anti-inflammatory $\mathrm{Ck}$ as molecular markers to identify patients at risk of future events and therefore most likely to benefit from endarterectomy (CEA); the relation of intracellular cytokine expression with the degree of carotid artery stenosis $(\geq 70 \%$ or $<70 \%)$, presence and type of clinical symptoms and plaque type.

Materials and Methods: Intracellular expression of proinflammatory cytokines TNF- $\alpha$, IFN- $\gamma$, IL-1 $\beta$, IL-6, IL- 8 and the anti-inflammatory cytokines IL-4 and IL-10 was determined in 106 patients (67 undergoing CEA and 39 not undergoing), before and after CEA in the first subgroup.

Results: Cells primed for the pro-inflammatory cytokines were found in significantly higher percentages in patients undergoing CEA than in patients who were not $(\mathrm{P}<0.05)$. Intracellular cytokine expression was significantly higher and diversified in patients undergoing CEA who had stenosis $\geq 70 \%$, with previous stroke and with amaurosis fugax than in patients not undergoing CEA.

Conclusion: Increased intracellular cytokines in patients' peripheral blood might be a warning signal indicating progressive atherosclerosis. If so intracellular cytokine monitoring could help in selecting patients at high risk of future clinical cardiovascular events and therefore most likely to benefit from CEA.
43

\section{A Case of Uncommon Liver Trauma Due to Chest Drain}

L. Fabris', M. Valmasoni', A. Pecchielan', E. Di Fratta', C. Longo ${ }^{1}$, A. Ruol', S. Nicotra ${ }^{2}$, M. Costantini ${ }^{1}$, E. Ancona ${ }^{1}$

${ }^{1}$ Department of Gastrointestinal and Surgical Sciences, Clinica Chirurgica I, University of Padova School of Medicine, Via Giustiniani 2, 35128 Padova, Italy,

${ }^{2}$ Cardiothoracic and Vascular Sciences Department, Thoracic Surgery Division, University of Padua, Padua, Italy

Introduction: We describe a major complication following the positioning of a chest drainage which resulted in a severe liver injury.

Materials and Methods: A 59-year-old man in $8^{\text {th }}$ postoperative day (POD) after right superior lung lobectomy required the insertion of a drain into the pleural cavity for idropneumothorax. This procedure presented some difficulties and the chest $\mathrm{X}$ ray revealed the drain, positioned in $9^{\text {th }}$ intercostal space, located in the abdomen. The drain was promptly removed. At our observation the patient was haemodinamically stable, with a peritonitic abdomen, agitation and dyspnea. The patient was immediately studied with an US scan and an AngioCT scan which demonstrated a liver haematoma ( $11 \times 9 \times 7,5 \mathrm{~cm}$ in $\mathrm{S} 6$ and S5) active liver bleeding and haemoperitoneum. The exploratory laparotomy revealed that the Argyle drainage penetrated the liver laterally in S6 and stopped near the gallbladder with Glisson capsule rupture. We obtained a good haemostasis by argon beam coagulator and liver packing. The AngioCT scan in first POD demonstrated no active bleeding and reduction of the haematoma. Because of an emerging haemodinamic instability, probably due to an inferior vena cava compression by the packing positioned under the liver and post surgical right diaphragm relaxatio we surgically removed the abdominal gauzes obtaining an immediate haemodinamic stability.

Results: The patient was discharged in 13th POD.

Conclusions: The blind positioning of an inferior pleural drain after lung surgery needs particular attention. 


\section{4 \\ Carcinoma of the Right Kidney with a Massive Neoplastic Trombosis of the Inferior Vena Cava: En-Bloc Resection without Vascular Reconstruction}

F. Farelli, R. Caronna, M. Schiratti, G. Prezioso, I. Cascioli, G. Fanello, M. Benedetti, F. Papini, R.L. Meniconi,

G. Dinatale, G.C. Russillo, L. Argentino, C. Benucci, P. Chirletti

"SAPIENZA" Università di Roma - Policlinico Umberto I Dipartimento di Chirurgia"F. Durante", U.O.C. Chirurgia

Generale N, Scuola di Specializzazione in Chirurgia Generale $V$ (indirizzo in Chirurgia d'Urgenza), Direttore

Prof. P. Chirletti

Introduction: Renal vein thrombosis is present in 19\% of renal tumors at diagnosis, while the involvement of the inferior vena cava (IVC) is observed in $7 \%$ of cases. Trombectomy with or without cavotomy shows 5 -year survival of $33 \%$ in patients with also nodal metastasis.

Materials and Methods: A 77 years old patient was admitted with a diagnosis of right renal carcinoma $(10 \times 12 \mathrm{~cm})$ with extensive inter-aortic-caval lymphadenopathy, infiltration of renal vein and inferior vena cava thrombosis (IVC) extending to the infrahepatic segment of the IVC for about $10 \mathrm{~cm}$. A RioBranco incision was performed. Vascular isolation of the IVC was achieved superiorly (infrahepatic) and inferiorly (common iliac veins) to the thrombus, a clampage of the proximal caval vein was performed. Hemodynamic stability was confirmed and monitored by intraoperative transesophageal echocardiography.

We performed a right radical nephrectomy with inter-aorticcaval lymphadenectomy and vena cava en bloc resection including the neoplastic thrombus. The ligation and section of left renal vein was performed downstream of the confluence of the spermatic vein. Cardiovascular stability and the presence of efficient collateral circulation led us to renounce the implantation of a IVC graft.

Results: The patient was discharged after 9 days and an MR after one month demonstrated the absence of residual disease and no dilatation of the common iliac veins resected. No edema of the inferior limbs.

Conclusions: The caval tumor invasion in patients with carcinoma of the kidney, although it is a negative prognostic factor, should not be considered an absolute contraindication to surgical treatment.
45

\section{Enhanced Recovery in Elective Colorectal Surgery: Results and Compliance Analysis in 85 Patients}

\section{Seccia}

U.O. Chirurgia Generale e d'Urgenza, Direttore Prof. M. Seccia - Azienda Ospedaliera Universitaria Pisana

Introduction: The way to approach colorectal surgery with a multimodal fast track protocol is now well defined but still not widely applied. We present our main data on functional recovery and patients' compliance with the protocol.

Methods: 85 patients submitted to colorectal resection with a fast track protocol. Exclusion criteria were emergency surgery, the need for a stoma, ASA IV and the inability to perform an epidural analgesia. Mean age was 63(35-78) with 41 females and 44 males.58 patients underwent laparoscopic surgery and 27 open surgery.

Results: The post operative length of stay based on a fit for discharge criterium was 5 days. Patients were able to feed with solid food on $3^{\text {rd }}$ day p.o. and to walk on $2^{\text {nd }}$ p.o. day. Bowel movements occurred at $3^{\text {rd }}$ p.o. In 14 cases $(16,5 \%)$ NGT was positioned for PONV episodes. Overall morbidity was 15,2\%. Readmission rate was $5,9 \%$ ( 5 cases) due to: anastomotic leakage (1), abdominal abscess (1) subocclusive episode (1) pleural effusion (1) and renal colic. Compliance with the protocol has been over $70 \%$ for all the FT items except for solid diet, scheduled on $2^{\text {nd }}$ p.o. day in which only $34 \%$ of patients were able to feed with solid food, and early mobilization, scheduled on $1^{\text {st }}$ p.o. day in which $54 \%$ were able to walk. Compliance was over $70 \%$ respectively at $4^{\text {th }}$ p.o. and $2^{\text {nd }}$ p.o. day.

Discussion: Fast track surgery is confirmed to be feasible with a good compliance and to allow a significant improvement in p.o. recovery and a better post operative course with a shorter hospital stay.

46

Inguinal Hernia. Laparoscopic Versus Open Surgery : A Meta-Analysis

F. Coratti, L. Senescende, R. Malatesti, L. Gazzabin, M. Bucalossi, A. Coratti, G. Baldoni, V. Borgogni, F. Tani, A. De Martino

Department of General Surgery, University of Siena, Hospital "Santa Maria alle Scotte", "Department of General Surgery, Grosseto, Hospital "Misericordia"

Introduction: In the last few years we assisted to a progressive diffusion of the surgical laparoscopic approach in the treatment of the inguinal hernia through two ways: transabdominal preperitoneal (TAPP) and totally extra peritoneal (TEP). The aim of this study consists in the critical analysis of the results of the laparoscopic and open surgery in the approach of the inguinal 
hernia through the meta-analytic revision of the principal checked prospective trialses.

Materials and Methods: From the revision of the literature, 12 studies emerged to a meta-analysis with a total of 5195 patients.

Results: Among the outcomeses we have observed, with statistic significant, a reduction of the operating time, a reduction of the postoperative hospitalization, a redoubt presence of seromas after intervention and a diminution of the urinary retention for the group open.

From these values, the laparoscopic group have advantages for the best postoperative residual sensibility and the absence of chronic pain.

The recidivists seem to be smaller in traditional surgery instead.

Conclusion: The laparoscopic hernioplastic is an advanced technique and it represents a valid alternative to the traditional intervention. The indications are limited: recidivous hernias, bilateral hernias, athletes.

\section{7 \\ Complicated Right Diverticular Disease: Case Reports and Review of the Literature \\ P. Tarchi, M. Kosuta, G. Giacomel, U. Ginanneschi, N. de Manzini \\ Department of General Surgery, University of Trieste, Trieste, Italy}

Introduction: The right colonic diverticular disease is a rare entity in the Western countries, where it involves the $5 \%$ of the population, while the prevalence in Asian country is up to $20 \%$.

Diverticula can be solitary, true and congenital in origin, or multiple, tipically acquired and false in nature. The clinical presentation can be really challenging to differentiate from an acute appendicitis. The differential diagnosis includes the IBD such as Crohn's disease, malignant neoplasms, acute cholecistitis and rare intestinal TB. The reported rates of correct prteoperative diagnosis vary from 4 to $16 \%$ in the Literature.

Materials and Metods: Radiological exams are mandatory to obtain a correct preoperative diagnosis. CT scan of the abdomen is the gold standard and allows to identify both intra and extraluminal signs of diverticulitis.

The treatment of simple acute right diverticulitis is manly conservative, infact, the patient's age and number of attacks are not considered indications to the elective resection, based on the more benign course compared to the left-side diverticular disease. The disease recurrence rarely needs to be treted with urgent surgery, so the elective resection is indicated only in strictly selected patients.

The cases of complicated diverticular disease must be trated with an urgent intervention, such as a right hemicolectomy. This procedure is advocated in every complicated case or when a malignant neoplasm is suspected.

Conclusions: We report two cases of complicated diverticular disease in young men presenting with palpable abdominal inflammatory masses and both caracterized by a difficult differentiatial diagnosis from colonic cancer.
48

\section{Increase Mas1 Oncogene-Receptor Gene Expression in Colon Cancer}

\author{
G. Giacomel, S. Palmisano, M. Giuricin, S. Bernardi,
} L. Buri, B. Fabris, N. de Manzini

Department of General Surgery, University of Trieste, Trieste, Italy

Background: Angiotensin-(1-7) is generated from ACE2dependent degradation of angiotensin II. The combination of angiotensin-(1-7) and Mas1 receptor has an antiproliferative effect on smooth muscle cells of blood vassels and cells of adenocarcinoma of the lung.

Aim of this study is to show the involvement of ACE2 and MAS 1 receptor in colon cancer carcinogenesis.

Methods: Between December 2009 and April 2010, 40 patients were enrolled in the study. 20 colon cancer patients underwent right/left-sided hemicolectomy or sigmoidectomy and tissue samples were obtained immediately after surgery (20 from tumor and 20 from the same surgical specimen $5 \mathrm{~cm}$ far from the tumor). The control group was represented by samples taken from 20 healthy patients undergoing diagnostic colonoscopy. From all samples, mRNA was extracted and quantitative real-time RT-PCR was performed.

Results: The mean age was 72 years old for colon cancer patients and 63 for control patients; in both groups 52\% were male. $33 \%$ of healthy patients vs. $47 \%$ of cancer patients were taking drugs with effects on the renin angiotensin system.

The gene expression analysis showed a statistically significant increase in tumor MAS1 compared to normal mucosa (1,23 vs $5,829$; p $<0,001)$, sensitivity $100 \%$, specifity $81 \%$, while no difference, in gene expression for ACE 2 was noted.

Conclusions: Our results show, for the first time, a statistically significant increase in the MAS 1 receptor in colonic carcinoma compared to normal mucosa, demonstrating the effective involvement of the axis ACE2 Angiotensin 1-7 and MAS1 receptor in colonic carcinogenesis. Studies' results are still pending.

49

Six-Year Outcomes of Laparoscopic Adjustable Gastric Banding, Laparoscopic Gastric Bypass and Sleeve Gastrectomy: A Retrospective Study

\author{
L. Paulatto, M. Giuricin, S. Palmisano, C. Nagliati, G . \\ Giacomel, A. Balani, N. de Manzini \\ Department of General Surgery, University of Trieste, \\ Ospedali Riuniti di Trieste, Italy
}

Background: The aim of this study is to compare outcomes between laparoscopic gastric bypass (LGB), laparoscopic adjustable gastric banding (LAGB) and sleeve gastrectomy (SG) with 6-year follow-up.
178

Eur Surg Res 2010;45:158-307 23rd National Congress of the Italian Society of Young Surgeon (SPIGC) 
Methods: We evaluated patient demographics, mortality, complications, weight loss and amelioration of obesity-associated complications in 118 bariatric procedures performed from 2004 to 2009 in our Department.

Results: We examined outcomes in 64 LAGB, 49 LGB and 5 SG procedures. The mean BMI was higher in SG group (52.8, $\pm 7.4)$ and in LGB group $(46.8, \pm 5.4)$ versus LAGB group $(43.6, \pm$ 4.9). In LAGB group 11 patients had complications, in LGB group 13 (1 death) and in SG group 1.

The percent excess-weight loss was 16.4, 24.9, 36.9, 41.9, 41.4, $40.9,45.0$ and 31.3 at $3,6,12,1824,36,48$ and 60 months postsurgery for LAGB patients; $34.4,49.9,60.0,66.6,57.2,55.7$ and 32.6 at $3,6,12,18,24,36$ and 48 months postsurgery for LGB patients and $29.4,42.5,43.6$, and 53.8 at 3, 6, 12 and 18 months postsurgery. 2 of 3 patients in LAGB group and 7 of 9 in the LGB group taking medications for type 2 diabetes preoperatively, could stop or reduce their treatment.

Conclusion: Our study suggests superior weight loss and improvement in obesity-associated complications, especially diabetes, for the LGB. The LAGB is effective if patient selection is done by a multidisciplinary team.

\section{0 \\ Four-Year Outcomes of Intragastric Balloon for Morbid Obesity: A Retrospective Study

\author{
M. Giuricin, L. Paulatto, S. Palmisano, C. Nagliati,
} \\ G. Giacomel, A. Balani, N. de Manzini \\ Department of General Surgery, University of Trieste, Ospedali Riuniti di Trieste, Italy}

Background: Our aim was to evaluate the tolerance and the efficacy of the BioEnterics Intragastric Balloon (BIB) in terms of weight loss.

Methods: This is a retrospective outcomes analysis of $23 \mathrm{BIB}$ from 2006 to 2009. The positioning of BIB was performed under conscious or unconscious sedation while removal was done under general anaesthesia. The BIB was filled with air and was removed after 6 months. We evaluated patient demographics, complications and weight loss.

Results: There were 8 men and 15 women, the mean age was 45,1 (SD 11.9, range 25-70), the BMI was 40.9, SD 4.9, the mean percentage of excess weight (EW) was 85.2, SD 25.4. Balloon placement and removal were uneventful. Five patients were lost of follow-up $(21,7 \%)$, the follow-up rate was $65.2,66.7,60.0,61.1$, 61.1 and $56.3 \%$ at $1,2,3,4,5$ and 6 months postplacement. The \%EWLoss was 10.4, 16.5, 20.3, 25.1, 26.9 and 31.1 at $1,2,3,4,5$ and 6 months postplacement.

Conclusion: The intragastric balloon is a safe procedure inducing weight loss in selected obese patients. It is a very valid option for those who are not suitable for bariatric surgery. The balloon can play a role in the preoperative treatment of obese patients who are to undergo elective surgery, by lowering mortality and morbidity risk. The opportunity of repositioning the device two or more times in the same patient, should be evaluated.

\section{1 \\ Citoreductive Surgery and Hipec: Feasibility and Tolerability of the Procedure}

\author{
G. Luigina, G. Marco*, B. Walter, C. Emanuel, L. Marina*, \\ D. Annibale \\ Department of Surgery, Section of General and \\ Emergency Surgery, S. Maria della Misericordia Hospital, \\ University of Perugia, Perugia, Italy
}

Purpose: The aims of this study are to analyze feasibility, safety and clinical response in patients with peritoneal carcinomatosis by different kind of tumor treated by CRS plus HIPEC using the cisplatin-doxorubicin and cisplatin-mitomycin-C combinations, and to evaluate the possibility of an adjuvant chemotherapy.

Patients and Methods: Since 2006 all patients presenting to our Institute with peritoneal carcinomatosis were evaluated for surgery plus HIPEC. The surgical approach was cytoreductive surgery and peritonectomy. Surgery is followed by hyperthermic intraperitoneal drug administration according to Sugarbaker. If possible, patients also receive adjuvant chemotherapy.

Results: A total of 19 patients were enrolled. Four patients underwent liver wedge resection. R0 and R1 resection were performed in $16(84 \%)$ and $3(16 \%)$ respectively. The median number of adverse events was 2,1 (range $0-5$ ). Seven patients had no adverse events. Five patients experienced systemic toxicity. Only 4 (12\%) grade 3 adverse events was described. Young people $(<65$ ys) and high $(\geq 90)$ Karnowsky Performance Status patients can benefit more than others of combined treatment. It is necessary a good selection before enrolling patients to hipec and citoreductive surgery to reduce complications.

Conclusion: In our experience, HIPEC appears feasible, well tolerated and it allows a good local control of the cancer in fit patients. It's insufficient to prevent distant relapses in particular when the patient is no chemo-responder.

\section{2 \\ Self-Expandable Metallic Stent for Treatment of Malignant Colorectal Strictures: Our Experience \\ F. Cantarella, L. Graziosi, E. Cavazzoni, S. Mosca*, V. Zavagno, E. Elia, P. Ricci, A. Donini \\ Department of Surgery, Section of General and Emergency Surgery, " Department of Radiology, S. Maria della Misericordia Hospital, University of Perugia, Perugia, Italy}

\section{Cantarella F. MD, francescocantarella@alice.it}

Background: Intrinsic obstruction caused by primary or recurrent adenocarcinoma and extrinsic invasion/compression due to pelvic malignancies represent the main causes for malignant colorectal obstruction. Most of the patients with acute or chronic large bowel obstruction are often in poor general condi- 
tion (severe dehydration, electrolytes imbalance, advanced age, co-pathology) facing high risks as surgical candidates [1]. SEMS allow rapid decompression of colonic obstruction reducing operative procedures of $23 \%$ and number of colostomies from $43 \%$ to $7 \%$ [2], which result in a worsening of the quality of life and higher costs. The end-point of our preliminary experience is to evaluate if colonic stenting is feasible for palliation treatment of malignant colo-rectal strictures.

Materials and Methods: From December 2007 to June 2009 at our Unit, seven patients underwent colonic stenting.

80 years old male (F.M.) suffered for cardiac ischemia and obstructive respiratory syndrome, affected by stenotic and metastatic sigma neoplasia (stage IV).

72 years old female (B.S.) affected of endometrial adenocarcinoma relapse infiltrating the left urether and the sigma.

68 years old female (P.P.) affected of locally advanced and metastatic adenocarcinoma of the recto-sigmoid junction (stage IV).

84 years old male (B.V.) admitted for bowel obstruction due to stenotic neoplasia of the recto-sigmoid junction with liver and pulmonary metastases (stage IV).

74 years old male (A.L.) affected of anastomotic relapse due to left colic neoplasia.

79 years old male (R.G.) affected of pelvic relapse and pulmonary metastases due to rectal neoplasia.

70 years old male (T.M.) affected of left colonic relapse with pulmonary and liver metastases (stage IV).

All patients received WallFlex ${ }^{\mathrm{Tm}}$ colonic stent. Prophylactic antibiotics were administered. Stent insertion was performed under fluoroscopic guidance.

Patients had a plain abdominal radiograph 48 hours later to confirm stent full expansion.

Patients were discharged after 72-96 hours.

Results: In all patients successful decompression, defined as complete relief of bowel obstruction as judged by clinical symptoms and radiographic observation, was achieved. No precocious or posthumous complications were observed.

The first patient died 1 month later for disease progression.

The second patient underwent adjuvant chemotherapy. At 9 months from stent insertion a CT exam showed disease progression, no sign of stent dislocation. The patient died at 16 months from stent insertion for disease progression.

The third patient underwent adjuvant chemotherapy, died at 5 months from stent insertion for disease progression.

The fourth patient didn't underwent adjuvant chemotherapy for considerable morbidity. Patient died at 12 months from stent procedure for disease progression.

The fifth patient died at 4 months from double stent insertion for disease progression.

The sixth patient died at 1 month from stenting for disease progression.

The seventh patient died at 1 month from stent insertion for disease progression.

Conclusions: Our experience suggests that colonic stenting in colo-rectal malignant strictures is safe and minimally invasive, allowing a colonic decompression in both intrinsic and extrinsic neoplasia, with a better quality of life, less morbidity, mortality and costs. It doesn't affect the median survival compared with surgery $[4,5,6]$.
53

\section{Tailored Laparoscopic Resection for Gastric GIST}

G. Grillone, F. Roscio, C. Bertoglio, A. De Luca, I. Scandroglio

Unità Operativa di Chirurgia Generale, Presidio

Ospedaliero "Galmarini" - Tradate, Azienda Ospedaliera

"Ospedale di Circolo" - Busto Arsizio

Introduction: Tailored resection of gastrointestinal stromal tumors (GIST) of the stomach has been widely reported to be successful. We report a minimally invasive approach in a patient affected by GIST of the greater curve.

Materials and Methods: A 47-year-old male was referred to our Department with a history of mild abdominal pain at the upper right quadrant and dyspepsia. His history was completely negative. US didn't show any pathological finding. Laboratory tests were within normal ranges.

Gastroscopy demonstrated a $2.5 \mathrm{~cm}$ submucosal mass at the greater curve of the stomach with intact mucosa above. EUS features were compatible with GIST.

Preoperative CT scan didn't find local tumor invasion or distant metastases, while barium meal confirmed the localization of the mass at the greater curve.

Results: We performed a totally laparoscopic procedure with patient in a supine split leg position. Four 10-mm ports were placed on upper abdominal quadrants. Intraoperative gastroscopy was performed to clearly identify the lesion.

After mobilizing the greater curvature, a tailored sleeve stapled transection was performed by EndoGIA $45-\mathrm{mm} / 3.5-\mathrm{mm}$ to remove the tumor with a safe margin of normal gastric tissue. Tumor mass was removed by an Endobag through the umbilical port.

Intraoperative gastroscopy allowed both to test the staple lines for leakage and the residual gastric volume.

Definitive histology showed gastric GIST with $<5 / 50 \mathrm{HPF}$ and microscopically negative margins.

Conclusion: Tailored laparoscopic resection for the treatment of gastric GIST is a safe and feasible option and results in both functional and oncologic satisfactory outcomes.

54

\section{Laparoscopic Splenectomy and Cholecystectomy. A Combined Approach in Hematological Disorders}

M. Guaglio, M. Garancini, L. Degrate, F. Bagnariol, S. Poli, M. Luperto, M. Scotti, F. Uggeri, F. Romano

Clinica Chirurgica 1, Ospedale San Gerardo - Dipartimento di Scienze Chirurgiche, Università Milano Bicocca

Aim: Laparoscopic splenectomy (LS) is the gold standard for the treatment of hematological spleen diseases. Gallstones are often associated with these conditions requiring a combined pro- 
cedure. We report our experience with concomitant LS and cholecystectomy.

Methods: 28 patients ( 16 females, 12 males) with a median age of 15 years (range 6-55) underwent combined procedures. Indication were gallbladder stones associated with hereditary spherocytosis (21 patients), talassemia (4) and ITP (3). patients were in semilateral right position with a 5 trocars technique and tilting table from left to right. Cholecystectomy was performed first.

Results: No patient required conversion to open technique or blood transfusions. Mean blood loss was $145 \mathrm{ml}$, mean operative time was $170 \mathrm{~min}$, mean spleen size was $14.5 \mathrm{~cm}$ and median lenght of stay was 3.5 days. No major complications occurred in our series and hematological results were optimal.

Conclusions: Combined laparoscopic approach was safe and effective for the treatment of cholelytiasis associated with hematological conditions.

\section{5 \\ Radioguided Occult Lesion Localisation (R.O.L.L.) in Oncoplastic Surgery in Breast Cancer}

\author{
A.V. Granai, O. Buonomo, E. lannelli, G. Sena, P. Orsaria, \\ M. Venza, O. Schillaci, S. Elia, G. Petrella \\ General surgery and senology, University of Rome "Tor \\ Vergata", Fondazione Policlinico PTV
}

Introduction: Oncoplastic surgery is an emerging approach in breast-conserving therapy.

ROLL was shown to be associated with a reduced excision volume and more accurate centricity of lesion within the surgical specimen. ROLL is a quicker, more accurate and patient-friendly technique, which provides better cosmetic results and a higher percentage of tumour free margins.

Methods: All the patients underwent breast cancer surgery and plastic procedure during the same operation. The tumour was removed with at least $1 \mathrm{~cm}$ of macroscopically free margin, en-bloc with the tumour. After removal of the specimen, the remaining breast tissue was bimanually explored in its deep and superficial surfaces, in order to individualize eventual occult lesions.

Results: There were 257 patients with invasive tumours and 61 with DCIS. Average size of the tumour determined in the pathology analysis was $12 \mathrm{~mm}$ (range 0-30), and 32 tumours were multifocal. Mean weight of breast tissue removed $198 \mathrm{~g}$ (range 20-2100 g). Assessment of excision margins showed complete excision of the tumour in 302 patients (95\%); focally involved margins with DCIS in 9 patients $(3 \%)$; and closed $(<2 \mathrm{~mm})$ margins in 5 patients (2\%). One patient with focally involved margins with DCIS underwent a total mastectomy. Mean age was 50 years (range 31-71).

Conclusion: ROLL allows for the precise localisation necessary to optimise the cosmetic outcome in breast conserving surgery without jeopardising sufficient free tissue margins. ROLL can also be used in conjunction with sentinel node biopsy (SNB).
For all these reasons, ROLL is an important development in breast cancer treatment.

\section{6}

\section{Risk Factors for Postoperative Mortality in Patients with Visceral Perforations: The Results of an Observational Study}

\author{
Y. Lambert, D. Marrelli, M. Di Martino, G. Di Mare, \\ S. Caruso, F. Bettarini, G. Corso, F. Mariani, A. Neri, \\ F. Roviello, E. Pinto
}

Department of Human Pathology and Oncology, Section of Surgical Oncology, University of Siena, Italy

Background: Visceral perforations are one of the main causes of death in emergency surgery; they may be due to several causes and involve general population at any age. Different mortality rates are reported in literature, depending on patient's general conditions, delay of diagnosis, causes of perforations and location in gastrointestinal tract.

The aim of the present observational study was to analyze the clinical outcome in a consecutive series of patients with primary or postoperative gastrointestinal tract perforations.

Methods: Data regarding 154 patients (80 males and 74 females, median age 69) with gastrointestinal perforations requiring urgent operation between 2001 and 2009 were stored on database. Appendiceal perforations were excluded. Clinical presentation, patient's general conditions, site and cause of perforation, surgical features and postoperative course were analyzed. Potential correlations between postoperative mortality (defined as death occurred during the hospital stay or within 30 days from operation) and patient-related, disease-related or treatmentrelated features were investigated.

Results: During the study period, 99 primary and 55 postoperative perforations required operation. The main causes of primary perforation were neoplasms (19 cases), peptic ulcer (17 cases) and diverticular disease (17 cases); in 13 patients an iatrogenic cause of perforation (mainly operative endoscopy) was observed. Stomach/duodenum was the most common site of perforation (29\%). Overall postoperative mortality was $21 \%$. Ischemic, traumatic and postoperative perforations were associated with increased risk of mortality, whereas low mortality rates were observed in perforations from peptic ulcer or IBD. When considering the site of perforation, proximal colon showed the lowest mortality, and stomach/duodenum the highest rates. Age of patients was the most important factor influencing mortality $(4 \%$ in patients younger than 60 years, $49 \%$ in octogenarians, $\mathrm{p}<0.001$ ), independently from the cause and site of perforation.

Conclusion: Visceral perforations are a frequent cause of urgent operation in general surgery, and are associated with high mortality rates. Age of patients is the most important predictor of clinical outcome, and prognosis is particularly severe in octogenarians. 


\section{7}

\section{Use of Dexamethasone in the Prevention of PONV After Thyroid Surgery}

\section{F. Feroci, M. Rettori, A. Borrelli, E. Lenzi, M. Scatizzi \\ UO Chiruria Generale, Ospedale "Misericordia e Dolce" \\ USL4 - Prato}

Introduction: Nausea with or without vomiting after thyroidectomy occurs with an incidence of $60-76 \%: P O N V$-and not pain-is in fact considered the main cause of discomfort of postoperative recovery. The goal of this study is to verify the effect that a single dose of $8 \mathrm{mg}$ dexamethasone before surgery may produce on PONV after thyroidectomy for benign pathology.

Method: Seventy patients who underwent thyroidectomy were randomized into two groups:

Group A:8mg/2mL dexamethasone given by intravenous injection with $100 \mathrm{~mL}$ of physiologic solution 20 minutes before anaesthesia induction;Group B: $2 \mathrm{~mL} \mathrm{NaCl} 0,9 \%$ in $100 \mathrm{~mL}$ of physiologic solution injected as above. The postoperative therapy was standardized. PONV was estimated with a 4 -grade scale (0-3) 8 , 24, 32, 48 hours after surgery. Pain was estimated with a Visual Analog Scale (VAS) from 0 (no pain) to 100 (worst pain ever). Subjective vocal function was estimated with a voice-VAS $(100=$ regular voice, $0=$ worst voice ever).

Results: Group A patients suffered from a minor nausea incidence and severity $(\mathrm{p}=0.0001)$. Group B patient complained more pain $(\mathrm{p}=0.008)$. No differences in the evaluation of the subjective vocal function $(\mathrm{p}=0.693)$.

Conclusion: $8 \mathrm{mg}$ of dexamethasone is a simple,safe,economic method to reduce incidence and intensity of PONV and pain after thyroidectomy,therefore it should be used as a routine method before this kind of surgery.

58

\section{Thyroid Metastasis from Carcinoma of the Colon: Case Report}

\section{A. Lucchi, F. Vandi, M. Salvi, J. Brandolini, P. Berti, \\ G. Garulli \\ Plastic Surgery-University of Modena and Reggio Emilia, Modena, Italy}

Introduction: The thyroid metastasis from carcinoma of the colon are very rare and the literature has reported 31 cases only. We report one case of thyroid metastasis from carcinoma of the left colon.

Case Report: A female patient of 67 years, had a left hemicolectomy with anterior resection of the rectum for an advanced local adenocarcinoma. Three years late she had a surgical operation of the right inferior lobectomy for a metastasis coming from a colic adenocarcinoma.

After one year the patient had a surgical operation for a laparoscopic cholecystectomy, discovering and doing a resection of a metastasis (coming from the primitive colic tumor) of the IV segment. One year later there was a lesion at the 5 hepatic segment and at the left lobe of the thyroid. The hystologic test with needle biopsy showed a morphologic case of a metastasis form with primary colic; it was suggested to have a total thyroidectomy and resection wedge of the metastasis at the $\mathrm{V}^{\circ}$ segment. The case anatomic-surgical and hystologic showed a thyroid metastasis from adenocarcinoma extended to the perithyroidea soft tissue and to the windpipe. So it wasn't done a further hepatic metastasictomy.

The Discussion: The incidence of the thyroid metastasis from the colon-rectus cancer is unknown. The diagnosis of thyroid metastasis from the colon-rectus cancer can be very often belated: in our case 5 years after the colon surgical operation. In the literature there are described time till 9 years.

The surgical treatment is suggested when there are symtoms tied to the pressure of the windpipe and the swallowing.

\section{9 \\ Utilization of NIM-Response 2.0 During Total Thyroidectomy: Our Experience and Preliminary Results}

\author{
A.M. Lucisano, F. Lazzaro, N. Innaro, R. Sacco \\ General Surgery, “Magna Graecia” University, Catanzaro, \\ Italy
}

Introduction: One of the worse complications during thyroid surgery is represented by recurrent laryngeal nerve lesion;therefore,it's evident the extreme utility of a system that could be confirm the correct view and the anatomical course of RLN to prevent its possible damage. We report our experience with NIM-response 2.0 system in a series of 59 thyroidectomies.

Materials and Methods: From September 2009 to March 2010,59 cases have been operated with the intraoperative help of NIM. For each patient,we carried out 2 relieves of RLN activity for both sides, respectively at the time of its view and at the end of ipsilateral thyroid lobe isolation.

Results: We've reported only 1 RLN unilateral permanent paralysis among 59 cases.

Conclusions: NIM-response system has helped to make easy the right identification of RLNs,protecting their function and obtaining so a great advantage for the patient both for a prospective reduction of operative time and for a less postoperative discomfort (due to the possibility of dysphonia). Moreover, the electromyographic confirmation of RLN activity has a primary importance to carry out an accurate neck lymphadenectomy for cancer. However, NIM remains a tool that can't replace surgeon's experience,because we believe that it must to be used not as an instrument of "research",but for confirming the presence and the course of RLN,expecially in more difficult cases. 


\section{0}

\section{Anterior Mesh Rectopexy in the Treatment of Rectal Prolapse: A Single Institution Experience}

\author{
R. Tarquini, G. Luglio, V. Celentano, G. Antonelli, \\ M.C. Giglio, V. Sollazzo, L. Bucci \\ Department of General Surgery, Surgical Coloproctology \\ Unit, Federico II University, Naples
}

Introduction: D'Hoore technique is a novel approach for the treatment of complete rectal prolapse. It is an abdominal procedure based on an anterior rectal mobilization followed by a mesh rectopexy. The mesh is fixed down in the rectovaginal space and upward to the sacral promontory, thus avoiding the posterior rectal dissection and any sigmoidal-rectal kinking.

Materials and Methods: 30 female patients were enrolled (mean age 55.7), starting from June 2005, and were prospectively followed for one year after surgery. Patients were randomly assigned in one of two groups: Group A (20 patients, control group, standard mesh rectopexy); Group B (10 patients, D’Hoore technique). Complete rectal prolapse was diagnosed through rectal examination, proctoscopy and defecography. All patients were preoperatively and postoperatively evaluated through clinical questionnaires: Wexner continence score, Wexner constipation score, Rome III criteria.

Results: All patients had a preoperative Constipation score $>15$. In Group A, 5 patients (25\%) also had a postoperative constipation score $>15$, due to persistent outlet syndrome. One patient (5\%) experienced a clinical recurrence, while in two patients (10\%) a proctoscopic recurrence was assessed. In Group $\mathrm{B}$, all patients got a postoperative constipation score $<15$ and all of them showed an improvement with regard to the incontinence score. No recurrences nor postoperative complications occurred in this group.

Conclusion: Anterior mesh rectopexy is a safe and effective approach for the treatment of complete rectal prolapse. The improvement in constipation and continence scores is probably due to a better nerve preservation and to the anterior mesh location without rectosigmoidal kinking.

\section{1 \\ Minimally Invasive Surgery for Gastric GISTs: A Combined Laparoscopic- endoscopic Solution}

\section{F. Torelli, L. Marano, M. Schettino, R. Porfidia, G.M. Reda, M. Grassia, B. Braccio, M. Petrillo, N. Di Martino \\ VIII Unit of General Gastrointestinal Surgery \\ (Chief: Prof. N. Di Martino) - Second University of Naples}

Gastrointestinal stromal tumors (GISTs) are the most common mesenchymal neoplasm of the digestive tract with an estimated actual incidence more than $0.4 \%$. Although the feasibility of minimally invasive resections of gastric GISTs has been established, many aspects of this approach are still debated: in different scientific papers, gastroscopy during laparoscopy seems to be an effective technique for successfull intraoperative tumor identification and it is considered essential for laparoscopic resection of subepithelial masses. A total of 7 patients were referred to our institution for gastric GISTs. Preoperative work-up for all patients included medical history, standard blood tests, upper gastrointestinal (GI) endoscopy with endoscopic ultrasound examination and computed tomography (CT) scan. 1 of these patients underwent open surgery for acutely presentation (hemorrhage), and the other 6 underwent laparoscopic-endoscopic "rendez-vous" resection. The average operative time was $142.3 \pm 64.9 \mathrm{~min}$. The mean estimated blood loss was a little less than $73 \mathrm{ml}$ (range, 10-157 ml). There were no episodes of tumor rupture or spillage, no major intraoperative complications and a conversion rate of $33.3 \%$. At a mean follow-up of 25 months (range, 3-41) all patients are alive and disease free. There were no local recurrences. Given these findings as well as the advantages afforded by laparoscopic-endoscopic rendez-vous, a minimally invasive approach should be the preferred surgical treatment in patients with small and medium-sized gastric GIST; moreover a selective approach to laparoscopic resection based on tumor location allows safe resection of these tumors with low morbidity and no compromise of oncologic principles.

62

\section{The Best Surgical Treatment of Symptomatic Large Gastric Diverticula}

L. Marano, M. Schettino, F. Torelli, R. Porfidia, G.M. Reda,

M. Grassia, B. Braccio, M. Petrillo, N. Di Martino

VIII Unit of General Gastrointestinal Surgery

(Chief: Prof. N. Di Martino) - Second University of Naples

Gastric diverticula are unusual entities, often asymptomatic, that may present with a variety of a vague abdominal symptoms, such as upper abdominal pain, nausea, emesis and dyspepsia. Moreover bleeding and perforation represent other rare symptoms. Nowadays the treatment of gastric diverticula is still debated: although medical treatment is recommended for small diverticula $(<2 \mathrm{~cm})$, surgical laparoscopic resection represents the best treatment on case of large $(>4 \mathrm{~cm}$ ) or complicated malformation. We report a successful laparo-endoscopic approach to 2 symptomatic gastric diverticula. The symptoms were unspecific, being upper abdominal pain, epigastric fullness, and early satiety. The patients were submitted to barium study, endoscopy, stationary manometry, 24 hours $\mathrm{pH}$-metry and electrogastrography. Diverticula (6 and $4 \mathrm{~cm}$ in length respectively) were localized to postero-lateral gastric wall: we performed a surgical laparo-endoscopic resection. Mean operation time was $85 \mathrm{~min}$ (range, 60-110 $\mathrm{min}$ ), the mean blood loss was $43 \mathrm{ml}$ (range 19-67ml), and the mean length of hospitalization was 5,1 days (range 4-7). There were no major perioperative complications or mortalities and at a 6-months follow-up they are symptoms free. In conclusions laparo-endoscopic resection is a feasible and effective treatment with excellent outcomes for large and symptomatic gastric diverticula. 
63

\section{Stapled Transanal Rectal Resection, Our Experience}

F.S. Mari, L. Masoni, R. Maglio, A. Milillo, A. Pancaldi, F. Pindozzi, A. Dall'Oglio, G. Berardi, A. Brescia

UO Day Surgery, Azienda Ospedaliera Sant'Andrea, Università La Sapienza di Roma - II Facoltà di Medicina e Chirurgia

Introduction: The obstructed defaecation syndrome (ODS) occurs in about $7 \%$ of adult population, specially in women up to fifty years of age and is related to the pelvic floor dysfunction, functional outlet obstruction, mechanical outlet obstruction and dissipation of force vector. Today the introduction of a new surgical device the Contour Transtar for the STARR has lead to a new treatment approach of ODS.

Methods: We perform from June 2007 to December 2009, 138 consecutive stapled transanal rectal resection with transtar for ODS. An RX defecography and anorectal manometry were performed before the intervention in all pts. and 19 pts underwent to a preoperative pelvic floor rehabilitation with biofeedback.

Results: An improvement of the clinical conditions and symptoms were observed in all cases. $77 \%$ of the pts. reduced the laxatives and enema use and all the women abandoned the digitalization. The main complications included 2 persistent pelvic pain, 1 anastomotic stenosis and 1 recto-vaginal fistula that required a surgical treatment.

Conclusions: The TRANSTAR procedure seems to be the ideal treatment in case of ODS when the rectal prolapse with recto-rectalis or recto-analis intussusception are present. A preoperatory study including RX defecography and anorectal manometry are mandatory.

\section{4 \\ Surgical Treatment of Rectal Outlet Obstruction with Internal Delorme Procedure}

\section{S. Martina, E. Ganio, E. Novelli}

Ospedale S.Giovanni Battista, Chirurgia Oncologica (Prof. Mussa), Torino

Introduction: Our experience with the internal Delorme procedure with or without levatorplasty for rectal outlet obstruction.

Material and Method: Retrospective study was carried out using a prospective date base of 95 patients treated from March 2002 to March 2009 with obstructed defaecation. Patients were selected by validated constipation and continence scorings, clinical examination, colonoscopy and defecography. 58 patients (Group 1) were treated by the internal Delorme procedure and in 37 (Group 2) was combined with a levatorplasty. Patients were evaluated using the Cleveland clinic constipation score (CCCS), straining-index and PAC-QoL. Mean follow-up period was 3.2 (0.2-6.9) years.

Results: Twenty-one $(22,1 \%)$ patients developed a postoperative complication, 15 in Group 1 and 6 in Group 2. Constipation improved $(\mathrm{p} \leq 0.001)$ with a reduction of the time to evacuate $(13,4$ vs $7,6 \mathrm{~min})$, the manual assistance ( $83 \%$ vs $6,1 \%)$ and laxative use $(100 \%$ vs $27,1 \%)(\mathrm{p} \leq 0,001)$. The CCCS score decreased from 11,32 to 4,17 ( $\mathrm{p} \leq 0.001)$ as did the straining-index ( $10,96 \mathrm{vs}$ 4,$26 ; \mathrm{p} \leq 0.001$ ). The number of evacuations increased from 5,97 to 8,51 per week $(\mathrm{p} \leq 0.001)$. Overall clinical outcome was considered favorable in $84,2 \%$ of patients. PAC-QoL showed a reduction of anxiety/depression, physical discomfort and psychological discomfort $(\mathrm{p} \leq 0.001)$.

Conclusions: The internal Delorme procedure is a treatment option for rectal outlet obstruction with excellent functional results and patient satisfaction. The risk of recurrent prolapse and morbidity is low.

\section{5 \\ Rose: Revisional Endoscopic Obesity Surgery}

R. Maselli, A. Genco, M. Cipriano, N. Basso

L’obesità è una delle patologie più diffuse. La terapia chirurgica è attualmente l'unica forma di trattamento che consenta la guarigione duratura; la chirurgia può essere gravata da insuccessi a lungo termine, con una ripresa ponderale che nel caso del by-pass gastrico arriva fino al 35\% a 5-10 anni dall'intervento; tali insuccessi sono nella maggior parte dei casi imputabili ad un aumento del diametro maggiore dell'anastomosi gastro-digiunale. Oggetto di crescente attenzione e sperimentazione sono le possibilità che potrebbero essere offerte alla chirurgia bariatrica e sue revisioni dalla N.O.T.E.S. tra le quali nuove tecniche si sutura. Da gennaio 2010 sono stati trattati 2 stomaci di maiale mediante lo strumento "USGI Transport" ed i devices specifici (g-Prox ${ }^{\oplus}$ Grasping/Tissue Approximation Device e g-Cath Tissue Anchor Delivery Catheter ed il g-Lix ${ }^{\text {Tx }}$ Helical Grasper) a simulare la restrizione anastomotica. Gli stomaci trattati sono stati posti in osservazione per 30 giorni in ambiente acido. La nuova metodica ha permesso la creazione di una sutura efficace, come dimostra la persistenza del sistema di ancoraggio dopo 30 giorni, in ambiente acido. Il Trasport sembra una metodica efficace per la revisione endoluminale (ROSE: Revisional Obesity Surgery, Endoscopic) di interventi bariatrici. Futuri studi "in vivo" saranno necessari per confermare la sua validità. 
66

\section{Spontaneous Cholecystocutaneous Fistula. A Case Report}

S. Mazzei, P. Acquaro, F. Tagliabue, M. Chiarelli,

G. Confalonieri, A. Romelli, M. Costa

Azienda Ospedaliera "A. Manzoni", Dipartimento di area Chirurgica, Lecco

Spontaneous cholecystocutaneous fistula was a common complication of chronic calculous cholecystitis until the beginning of the twentieth century. External biliary fistulas or abscesses rarely occur spontaneously as a result on intrahepatic abscess, necrosis or perforation of the gallbladder, or other inflammatory process involving the biliary tree. Most cholecystocutaneous abscess or fistulas are post operative complications of liver and biliary tract surgery or trauma.

A 81-years-old female patient was admitted to our Emergency Department complaining of erythematous swellings with a little hematoma of the lateral abdominal wall and pain of the right upper quadrant. The physical examination revealed a abdominal wall abscess in the right subcostal area surrounding cellulitis. The white blood cell count was $8,500 / \mathrm{mm}^{3}$. The ultrasonography and computed tomography (CT) identified an abscess formation.

Cutaneous external fistula is a rare presentation of cholelithiasis. In the pathophysiology of perforation plays an important role the obstruction of the cystic duct caused by calculus or carcinoma. The external fistula more frequently drain to the right superior quadrant and right flank or to the umbilicus.

Emergency laparotomy is the correct therapeutic option in septic patients. Instead an elective open cholecystectomy with the excision of the fistulous tract appears to be the standard approach in all other patients.

\section{7 \\ Gallbladder Metastasis of Renal Cell Carcinoma: A Case Report}

S. Mazzei, F. Tagliabue, P. Acquaro, M. Chiarelli, M. Origi,

G. Confalonieri, A. Romelli, M. Costa

Azienda Ospedaliera "A. Manzoni", Dipartimento di area Chirurgica, Lecco

Introduction: Metastasis from renal cell carcinoma (RCC) are frequent to distance organs, in particular to the lung, liver, and bone, however metastasis to the gallbladder is extremely rare: it's most often detected incidentally at autopsy. The incidence of gallbladder metastasis was reported to be $0.57 \%$ ( 3 out of 523 autopsies) in a study by Bennington et $\mathrm{Al}$, and $0,58 \%$ (4 out of 687 autopsies) by Weiss et $\mathrm{Al}$.

Materials and Methods: In March 2006 a 73-years-oldman was admitted in Casualty complaining of abdominal pain, fever and jaundice. He referred nephrectomy in the 2001 for RCC. The patient underwent clinical and radiological exams that relieved gallstones and choledocholithiasis associated with a $1 \mathrm{~cm}$ polyp of the mucosa of the gallbladder. He was treated with ERCP. Three months later he underwent laparoscopic cholecystectomy. The hystological examination revealed that the submucosal polyp was a clear cell carcinoma consistent with metastatic RCC, which was morphologically similar to the renal tumour excised in 2001. The cells were positive for CD 10 and vimentin, but negative for CD 68, cytokeratine 7 and 20.

Conclusion: Due to the low response to chemotherapy, surgical management in selected patients with metastatic RCC should be considered. The criteria for the selection of patients for aggressive surgical management are not well defined.

68

\section{Abdominal Compartment Syndrome Due a Giant Multilobulated Ovarian Serous Cystadenoma}

\author{
D. Merlicco, P. Villani, S. Rucci, S. Schirone, S. Massa, \\ F. Altilia, G. Roggia \\ U.O.C. di Chirurgia Generale, Presidio Ospedaliero di \\ Lucera (Fg) ASL Fg
}

Introduction: Woman of 47 years, virgin, about 24 months relates a progressive abdominal distention to procure an Abdominal Compartment Syndrome grade II with a IAP of 16 mmhg, cardio-respiratory impairment (FC $100 \mathrm{~b} / \mathrm{m}$ and FR 18/ $\mathrm{min})$, dyspnea in supine position $\left(\mathrm{sO}^{2}: 89 \%\right)$, the contraction of diuresis for several days.

Materials and Methods: The patient arrives in emergency with the diagnosis of acceptance for abdominal colic and dyspnea in supine position for about a week; the clinical objectivity seems to prove to a pregnant woman at term gestation. A spiral CT abdomen-pelvis shows a giant neoformation hypodense, fluid density, homogeneous, regular and thin-walled and size of $53 \times 45 \times 33$ $\mathrm{cm}$, occupying the entire abdominal cavity and displaces the compressed viscera in subdiaphragmatic and in the pelvis, and another cyst, multisetted, size equal to $30 \times 18 \times 14 \mathrm{~cm}$.

Result:: At laparotomy this neoplasm appears giant, multilobulated, totally cystic, ovarian vascular smooth start to the left ovary; it is between the symphysis pubis and the diaphragm and has dimensions of $62 \times 50 \times 45 \mathrm{~cm}$.

Left oophorectomy was performer; the tumor weighing $37 \mathrm{~kg}$ contains 31 liters of liquid blood serum; part of the cyst fluid s taken for cytologic examination (blood smears with some inflammatory cells) and partly for bacterial culture (no bacterial growth).

Conclusion: The authors present a case of ACS from a giant ovarian serous cystadenoma; it is one of the largest ovarian serous cystadenoma reported in the literature. The authors recommend performing an emergency exploratory laparotomy in patients with ACS Grade II and then to treat this benign disease as an acute abdomen. 
69

\section{The Use of Abdominal Flaps for Breast Reconstruction}

L. Canta, M. Moio, G. Molea

Cattedra di Chirurgia Plastica, Ricostruttiva ed Estetica,

Direttore: Prof. G. Molea, Università degli Studi di Napoli

"Federico II"

Introducrion: The mammary reconstruction pursues the obtainment of a breast with shape, volume and consistency similar to the normal breast. It can be achieved by using the own patient's tissues and/or alloplastic implants. The most appropriate technique is choosed after the assessment of the general conditions, age, anatomy, remnant tissues and expectations.

Materials and Methods: The lower abdominal region offers a multitude of flaps for breast reconstruction. The TRAM flap procedure uses skin, fat and muscle from the lower abdominal wall. It can be pedicle, leaving the flap attached to its original blood supply (perforators of the Superior Epigastric vessels) and tunnelling it under the skin to the breast area, or free, requiring the use of microsurgery to connect the vascular supply to the internal mammary or toracodorsal vessels. The DIEP flap only requires the removal of skin and fat. No muscle is sacrificed. The blood supply comes from the perforator arteries of the deep inferior epigastric vessels. The flap is connected to the patient's chest using microsurgery.

Results: The TRAM flap procedure allows to shape a soft and natural breast with results that mimic a "tummy tuck" procedure.

The DIEP flap has significantly fewer abdominal complications and side-effects (pain, bulging, hernia, strength loss) because the majority of the abdominal muscle is spared.

Conclusions: Breast reconstruction is fundamental for the acceptance of mastectomy, improving the patient's self image and confidence. The abdominal area is an ideal donor site for mammary reconstruction. An adequate planning and patient selection can achieve the best results choosing the most adequate technique.

70

\section{Extrapleural Malignant Solitary Fibrous Tumor Originating from the Mesentery}

\author{
M. Muroni, C. Cacchi, G. Cancrini, A. Cancrini, G. Mezzetti \\ La Sapienza University of Rome, Second School of \\ Medicine, St. Andrea Hospital, Rome, Italy
}

Introduction: Solitary fibrous tumor represent a rare soft tissue tumor and it was initially considered limited to the pleural cavity. More recently, however, it has been described in many extrapleural sites including the visceral organs, the retroperitoneum and the pelvic space.

Materials and Methods: We report a case of a 73-yearold Italian man hospitalized for abdominal pain and a palpable abdominal mass. An abdominal ultrasonography revealed a well- circumscribed solid tumor (25 x $20 \mathrm{~cm}$ in diameter) containing cystic areas. CT scan demonstrated a well-defined solid tumor. The patient underwent surgical treatment. At laparotomy, an elastic soft tumor was found originating from the mesentery and spreading to the pelvic space. The tumor had a vascular pedicle ranging from $5-6 \mathrm{~cm}$ in length. There were no disseminated nodules and no liver metastasis. Complete surgical resection of the neoplasm was performed.

Result: The resected specimen consisted of a solid and cystic part and was $25 \times 20 \times 10 \mathrm{~cm}$ in size Histopathological evaluation and immunohistochemical analysis was performed. The neoplasm was composed of high cellularity, roundish tumor cells arranged around ectatic vessels, high mitotic activity (22 mitosis/10 HPF). Immunohistochemical studies revealed a positivity for CD34, CD99 and Bcl-2. An absence of staining for cytokeratin, actin, desmin, S-100 protein was observed. On the basis of the results, pathological evaluation showed the presence of a malignant solitary fibrous tumor.

Conclusion: Malignant solitary fibrous tumor originating from the mesentery is extremely rare neoplasm in adult patients. Precise diagnosis is based on the correct interpretation of pathological and immunohistochemical features. Complete surgical exeresis remains the treatment of choice and a long follow-up is mandatory because of the possibility of late recurrence.

71

\section{Surgical Management of Hepatocarcinoma Chest Wall Metastasis: A Case Report}

\author{
F. Attene, F. Pulighe, P. Paliogiannis, F. Serventi, \\ G. Giangrande, A. Marrosu, F. Scognamillo, E. Trignano, \\ M. Trignano \\ Institute of Surgical Pathology, University of Sassari, Italy, \\ Università degli Studi di Roma "Tor Vergata" - Cattedra di \\ Chirurgia Oro-Maxillo-Facciale, Scuola di Specializzazione \\ in Chirurgia Odontostomatologica, Direttore Prof. \\ Leonardo Calabrese
}

Introduction: Metastatic tumor are the most frequent neoplastic lesions of the thoracic wall. We present a case of hepatocarcinoma metastasis, the surgical strategy adopted and a brief review of the literature on the management of such conditions.

Case Report: A 73 years old woman affected by hepatocarcinoma (HCC) and a mass in correspondence of the right chest wall was admitted in our department. The physical examination showed the presence of painful swelling of the right hemithorax and absence of pulmonary signs. Pre-operative chest $\mathrm{X}$ ray and CT scan showed a $46 \mathrm{~mm}$ in diameter mass which involved three ribs (6th,7th and 8th), the correspondent intercostal muscles and the adjacent fat tissue. The patient underwent ultrasound guided FNA which confirm the metastatic nature of the lesion derived from a primary hepatocarcinoma. After appropriate physical assessment, surgical exeresis of the mass and reconstruction of the chest wall with a Goretex prosthesis was performed. The specimen pathologic examination demonstrated surgical margins free of tumor invasion. No post - operatory complications were verified and the patient was discharged 8 days after surgery. 
Conclusions: The right preoperative assessment, the right choice of the prothesis and the optimal surgical strategy are crucial factors for an effective chest wall metastasectomy and reconstruction.

72

\section{PTH Serum Levels for the Evaluation of Hypoparathyroidism After Total Thyroidectomy \\ P. Paliogiannis, F. Attene, C. Torre, F. Cabras, \\ D. Giangrande, F. Serventi, A. Marrosu, F. Scognamillo, \\ M. Trignano \\ Institute of Surgical Pathology, University of Sassari, Italy \\ Specialità: Chirurgia Generale, \\ Descrizione abstract: Università degli Studi di Roma «Tor \\ Vergata», Cattedra di Chirurgia Oro-Maxillo-Facciale, \\ Scuola di Specializzazione in Chirurgia \\ Odontostomatologica, Direttore Prof. Leonardo Calabrese}

Introduction: Post-thyroidectomy hypoparathyroidism represents a cause of prolonged hospital length of stay. We evaluate the post-operative PTH serum levels as a predictor of hypoparathyroidism in order to guarantee an early and safe discharge of the patient.

Materials and Methods Forty patients, all with regular preoperative calcium serum and $\mathrm{PTH}$ values, were included. The PTH serum levels were measured one hour after surgery and the calcium serum levels were evaluated 12, 24 and 48 hours after the operation. The cut off PTH level studied in terms of sensibility, positive and negative predictive value was $10 \mathrm{pg} / \mathrm{ml}$.

Results The patients with prolonged hypocalcemia were 14 (35\%). 12 of them $(85,7 \%)$ had a post-operative PTH lower to 10 $\mathrm{pg} / \mathrm{ml}$, while $2(14,3 \%)$ where hypocalcemic but with PTH higher to $10 \mathrm{pg} / \mathrm{ml}$. In thirteen patients PTH was lower to $10 \mathrm{pg} / \mathrm{ml}$ and 12 of them $(92,3 \%)$ presented hypocalcemia within the first 48 hours after thyroidectomy; 26 patients (65\%) had normocalcemic values and in only 1 (4\%) PTH was lower to $10 \mathrm{pg} / \mathrm{ml}$. The patients with PTH higher to $10 \mathrm{pg} / \mathrm{ml}$ who presented hypocalcemia were 2 (5\%).

Conclusions: Data showed that postoperative PTH serum level using a cut off of $10 \mathrm{pg} / \mathrm{ml}$ have a sensibility of $85,7 \%$ to predict hypocalcemia, while the positive predictive value is $92,3 \%$ and the negative predictive value is $92,6 \%$.

\section{3}

\section{SILS Between Present and Future}

A. Paoloni, R. Pellerito, A. Buonsanto, N. Rocci, A. Salvucci, G. Catalini, O.C. Camerino

Introduction: The standardization of laparoscopic techniques with a drastic reduction of postoperative morbidity has led to an additional input to finding ways in which laparoscopic field can not be ignored by an ever closer union between modern design it technology and its applications in surgery.

In the light of a vision born increasingly invasive SILS (single incision laparoscopic surgery) with the idea of obtaining the same results by performing surgical access transombelicale one whose benefits are not purely aesthetic but also linked to reduced drug therapy postoperative analgesic and its possible applications in cancer surgery and robbotica.

Materials and Methods: Our experience with the SILS started in 2008 by submitting, at present, cholecystectomy with single-access 27 patients ( 4 men, 23 women).

Inclusion criteria of patients undergoing cholecystectomy with SILS technique were as follows below:

a) lack of xipho-umbilical scars from previous surgeries or subcostal

b) the presence of obesity, choledocholithiasis with jaundice, umbilical hernia or previous repair of an umbilical hernia, under acute cholecystitis clinical-instrumental

c) ASA 3

d) patients aged between 18 and 70 years

e) no sonographic evidence of cholecystitis sclerosus and / or suspicion of carcinoma of the gallbladder

Results: The average stay was 3 days with reduction of postoperative analgesics, and a good aesthetic result of the surgical scar. with an immediate conversion only for the presence of visceraatrophic gallbladder with anatomical structures difficult to identify. We had no complications in postoperative patients.

Conclusions: Based on our experience we found a reduction of postoperative pain with an aesthetically pleasing result. Randomized clinical trials currently underway will provide further information on the future of SILS technique in relation to the further development of the existing device with any potential development in cancer surgery and/or robbotica.

\section{4}

\section{Pneumomediastinum and Pneumoperitoneum After Blunt Chest Trauma: The Macklin Effect}

\author{
F. Passafiume, L. Valesini, D. Crocetti, L. Simonelli, \\ V. Cozza, M. Assenza, C. Modini \\ Dipartimento di Emergenza Urgenza, Sapienza Università \\ di Roma, Azienda Policlinico Umberto I. Rome, Italy
}

Introduction: Pneumomediastinum creation, in blunt chest trauma, results from Mackin effect in more than $95 \%$ of cases. This study underlines the relationship between Macklin effect, pneumomediastinum and pneumoperitoneum. It analyses conservative and invasive treatment.

Materials and Methods: We report two cases of blunt chest trauma with Pneumomediastinum creation and no evidence of airway lesions.

Results: The first patient presented malignant pneumomediastinum and pneumoperitoneum with Intra-abdominal hyperten- 
sion. A mediastinal drainage was placed and a laparostomy was created to prevent abdominal compartment syndrome.

The second case showed how Macklin effect can be self-limiting. This patient underwent conservative treatment.

Conclusions: In any case of post-traumatic pneumomediastinum, the Macklin effect should be suspected. Clinical observation is the treatment of choice since Macklin effect is selflimiting.

However, there are some life-threatening event, like malignant pneumomediastinum and Intra-abdominal hypertension, that must be relieved and treated immediately.

75

\section{The Role of Three-Dimensional Transrectal Ultrasonography in the Modification Stage of Rectal Cancer}

\section{A. Patrizi ${ }^{1}$, M. Guerrieri ${ }^{2}$, I. Patrizi ${ }^{1}$ \\ ${ }^{1}$ U.O. Chirurgia Generale, ASUR ZT-9 Macerata, ${ }^{2}$ Clinica di Chirurgia Generale e Metodologia Chirurgica - Ospedali \\ Riuniti Ancona}

Introduction: This study is performed to verify the usefullness of 3D TRUS (3D-Transrectal Ultrasonography) in the surgeon's hands in the modification stage of rectal cancer after neoadjuvant therapy and to compare these results to others common preoperative diagnostic instruments analized by most important and recent literature.

Materials and Methods: 40 patients with rectal cancer (11 Stage I, 16 Stage II, 13 Stage III) were enrolled in 2009. All patients have been underwent preoperative chemo-radiotherapy followed by local excision (by means of transanal endoscopic microsurgery) or by total mesorectal excision; also everybody have been studied pre- and post-neoadjuvant therapy with 3D-TRUS and MRI or CT. After operation we have compared the pre-operative stadiation with hystological reports.

Results: We have observed 22,5\% TRG1, 25\% TRG2, 20\% TRG3 and 32,5\% TRG4 sec. Mandard; the 3D-TRUS has demostrated these attendibility parameters after neoadjuvant therapy: Accuracy 75\%, Sensibility 92\%, Specificity 43\%, Positive Predictive Palue 75\%, Negative Predictive Value 75\%.

Conclusions: The better accuracy value of 3D-TRUS than 2D-TRUS after neoadjuvant therapy can be due to the possibility to observe the infiltration front throught three different prospective plan. This exam consents to optimize the surgical approach, local excision or total mesorectal excision, in the modification stage of rectal cancer, especially if performed or followed by the same surgeon who performs the operation.

\section{6}

\section{Orchidofuniculectomy and Amputation of the Penis for Fournier's Syndrome. Case Report}

\author{
D. Pisaniello, P. Ferravante, S. Coletta, F. Pacifico, \\ M. Carola, D. Scrocco
}

Divisione di Chirurgia Generale, Ospedale "Sacro Cuore di Gesù"- Fatebenefratelli - Benevento

Introduction: Fournier's gangrene is an acute bacterial infection of perineum and of the lower trunk causing "necrotizing fasciitis", accounting for 0,1-0,4\%/100.000 inhabitants . Alcool and diabetes are predisposing factors. The Authors report the case of a patient affected by Fournier's gangrene presenting wide extension of the necrosis and treated by surgical amputation of the penis, testicles and scrotum.

Case Report: Male, 81 ys., diabetes and paraplegy. Perianal fistula one month before. He came in emergency in poor clinical conditions, metabolic acidosis, kidney failure, fever $\left(39^{\circ} \mathrm{C}\right)$ with presence of extensive skin necrosis and subcutaneous enfisema involving perineum, scrotum, testis and penis spreading up to the trunk with the typical fetid smell, resembling a Fournier's gangrene. CT scan of the abdomen showed no concomitant pathologies. The patient underwent bilateral orchidofuniculectomy with amputation of the penis. An epicistostomy was also performed. Reconstruction of the perineum was performed by flaps and a drainage was kept in place for 6 days. Patient underwent daily washing and disinfection with saline solution and Betadine ${ }^{\bullet}$ Antibiotics were administered iv (Levoxacin $250 \mathrm{mg} / 12 \mathrm{~h}$ ). Four months after surgery the patient is in healthy condition and skin wounds completely healed.

Conclusions: Fournier's syndrome is to be considered a surgical emergency, because it can progress unfavourably in few hours, often causing severe skin necrosis and septic shock. Mortality ranges between 0 and $81 \%$, thus a prompt surgical treatment which provides complete and wide necrosectomy together with restore of metabolic conditions can save the life of the patient.

\section{7 \\ Posterior Double-Layer Technique: A New Proposal for Pancreatolejunal Anastomosis Following Duodenopancretectomy}

\author{
E. Poli, G. Lissidini, A. Gurrado, G. Piccinni, L. Ruggiero, \\ M. Testini
}

Introduction: Anastomosis of the pancreatic remnant with jejunum after partial pancreatoduodenectomy (PD) is a challenging practice. Several methods for pancreatojejunostomy (PJ) have been described, and end-to-side, telescopic, and duct-to-mucosa anastomoses, are usually the most frequent PJ reported.

Materials and Methods: We propose a new technique for end-to-side pancreato-jejunal anastomosis performing a double 
layer suture on the posterior side. The pancreatic duct is everted and tagged to periductal parenchyma with three-four interrupted 4/0 PDS-II sutures. At first a suture of the posterior pancreatic capsular edge to the posterior seromuscolar wall of the jejunum is performed with an interrupted 2/0 Ti-cron suture; a second layer of suture is applied anteriorly to the previous suture line excluding the lumen of Wirsung duct. Finally, the anterior layer is directly sutured with interrupted 2/0 Ti-cron sutures.

Results: We performed this innovative technique in two patients, undergone PD for head adenocarcinoma $(M, 82)$ and duodenal adenocarcinoma (F, 79). Morbidity and mortality were none.

Conclusion: Posterior double layer is a proposable technique that could reduce the risk of pancreatic fistula and anastomotic dehiscence reinforcing the posterior layer that is the most declivous site in the first post-operative days, when the patient lays in bed. "Evagination" of pancreatic duct prevents pancreatic ductal stenosis. This technique should be proposed especially in high risk patients with soft parenchyma because the functional integrity of the gland gives a higher capacity to destroy itself through acute pancreatitis and the anastomosis is technically more difficult.

\section{8 \\ Current Role of Intraoperative Ultrasonography in Liver Resection for Colorectal Metastases}

G. Ranucci, C. Mele, G. Sarno, F. Ardito, M. Vellone, F. Giuliante, G. Nuzzo

\section{3rd Classified - SPIGC Award Session, General Surgery}

Background: Liver resection is the gold standard for the treatment of colorectal metastases (CRM). In the last 10 years intraoperative ultrasonography (IOUS) and contrast-enhanced IOUS (CE-IOUS) have increased their role for correct intraoperative staging. Their accuracy compared to that of computed tomography $(\mathrm{CT})$ and magnetic resonance $(\mathrm{MR})$ is still debated. The aim of this study was to evaluate the impact of IOUS and CE-IOUS on surgical strategy and outcome.

Patients and Methods: Between 2000 and 2009, 266 patients with CT/MR diagnosis of CRM underwent liver resection with IOUS. In 66 patients (24.8\%) CE-IOUS was also performed.

Results: Relevant findings differing from those of preoperative imaging studies (greater/smaller number of CRM, proximity of CRM to vascular structures, involvement of vascular structures, more extensive disease) were assessed in 125 resections by IOUS and, in particular, in 33 of them by CE-IOUS $(33 / 66=50 \%$ of the totally performed CE-IOUS). Additional hepatic tumors were identified in 18 resections by CE-IOUS $(18 / 66=27.3 \%$ of the totally performed CE-IOUS), and in 39 resections by IOUS $(39 / 200=19.5 \%$ of the totally performed IOUS). The mean number of metastases identified by CT or MR, IOUS and CE-IOUS was 2.4 (median 2.0), 2.7 (median 2.0) and 3.2 (median 3.00), respectively.
A modification in the planned operation occurred in 51 resection (19.2\%) (IOUS: $34 / 200=17.0 \%$; CE-IOUS: $17 / 66=25.8 \%$ ).

Conclusion: IOUS and CE-IOUS are more effective than CT or MR to define number and features of CRM, and to guide liver resection. This is particularly evident when CE-IOUS is employed.

\section{9}

\section{Portal Vein Embolization and Major Hepatic Resections: A Single Center Outcome Evaluation in 62 Patients}

\author{
F. Ratti, F. Cipriani, C. Soldati, M. Catena, M. Venturini, \\ L. Aldrighetti \\ Dipartimento di Chirurgia Generale e Specialistica - Liver \\ Unit, IRCCS H San Raffaele, Università Vita-Salute San \\ Raffaele, Milano, Italy
}

Introduction: The extent of liver resection is limited by the residual functional reserve of the liver (FRL). The introduction of portal vein embolization (PVE), with the rationale of inducing compensatory hypertrophy of the FRL has significantly reduced morbidity and in particular the impact of "small for size syndrome" (SFSS). Objective of the study is to evaluate the feasibility and effectiveness of PVE in patients candidates for right hepatectomy at high risk of SFSS.

Materials and Methods: Between January 2006 and December 2009, 62 patients suffering from primary or metastatic liver tumor, underwent PVE at the Department of Surgery - Liver Unit HSR. CT assessment of hepatic volume was performed in each patient, prior and 4 weeks after the procedure. The outcome was evaluated in terms of feasibility of surgery, increasing FRL (calculated as: [FRL after PVE - PVE FRL pre] x 100 / FRL pre PVE), morbidity and mortality associated with PVE and surgery.

Results: Of the 62 patients undergoing PVE, 6 (9.7\%) have not benefited from further surgery: of these, 4 showed spread of disease in the FRL to CT control, while in the remaining 2 adequate compensatory hypertrophy was not reached. The average volume of the FRL at the time of the procedure and after 4 weeks was respectively $437.03 \mathrm{cc}( \pm 172.54)$ and $615.15 \mathrm{cc}( \pm 187.49)$ with an average increase of $50.3 \%( \pm 30.31)$. During the postoperative period only 2 patients $(3.2 \%)$ showed mild and transient signs of the SFSS.

Discussion: The technique of PVE makes it possible to performe, in an effective and safe way, major liver resections in patients with high risk of SFSS. 
80

\section{Stapled Haemorrhoidopexy: A Single-Center Experience with 787 Cases}

\author{
A. Noceti, E.L. Ratto, A. Rota, F. Maritato*, C. Eretta*, \\ A. Piccardo \\ S.C. Chirurgia Generale- Direttore Prof. A. Piccardo, ASL2 \\ Savonese Ospedale di Cairo Montenotte - Ospedale di \\ Savona, "S.S. Pronto Soccorso Ospedale di Cairo \\ Montenotte - Responsabile dott. F. Maritato
}

Introduction: Anorectal symptoms involve 50\% of western people. In USA 10 million people suffer of haemorrhoidal disease, with an incidence rate of $4.4 \%$. According with SICCR guidelines grade I should be treated conservatively, grade II: elastic ligature, HAL/T.H.D, Stapled Haemorrhoidopexy, sclerotherapy and photocoagulation; Grade III: Stapled Haemorrhoidopexy Haemorrhoidectomy, HAL/T.H.D, elastic ligature; grade IV Haemorrhoidectomy, Stapled Haemorrhoidopexy, (Ultracision, Ligasure)

Materials and Methods: From 1999 to 2010, we have performed Stapled Haemorrhoidopexy in 787 patients, 367 male and 420 female, with haemorrhoidal disease with mucosal prolapse of IIrd and IIIth degree. All operations have been conduced in locoregional anesthesia. Till 2003 we have used $33 \mathrm{~mm}$ circular stapler Ethicon Endo Surgery PPH 01; from 2004 PPH 2003. Follow-up was at 5 years in 380 pts (48\%); at 2 years in 190 pts (24\%); at 1 year in 115 pts (14.6\%); at 6 months in 60 pts (7.6\%).

Results: Middle operating time: 20 minutes. Hospital stay: one day surgery in 99,7\%. Pain evaluation (VAS scale 0-10): 2,1 in I postoperative day; 2,4 during first defecation. Analgesia in first 18 hours: 1 administration in 64\%; 2 administrations in $24 \%$; 3 administrations in $12 \%$. No opioids. Immediate complications: bleeding (1\%), urinary retention (1.7\%). Late complications: chronic pain $(0.3 \%)$, chronic bleeding $(1.7 \%)$, submucosal abscesses $(0.1 \%)$, recurrence $(6 \%)$.

Conclusion: We want to underline the lower rate of complications, the reduction of postoperative pain and the shorter hospitalization than Haemorrhoidectomy. The higher recurrence rate can be related to underestimation of the prolapse. Currently new techniques can be performed to manage important prolapse: STARR and TranSTARR.

\section{1}

Laparoscopic Ventral Hernias Repair: A Single-Center Experience with 203 Cases

\author{
A. Noceti, E.L. Ratto, A. Rota, F. Maritato*, C. Eretta*, \\ A. Piccardo \\ S.C. Chirurgia Generale- Direttore Prof. A. Piccardo, ASL2 \\ Savonese Ospedale di Cairo Montenotte - Ospedale di \\ Savona, "S.S. Pronto Soccorso Ospedale di Cairo \\ Montenotte - Responsabile dott. F. Maritato
}

Introduction: Our division performs laparoscopic treatment of primary and secondary ventral hernias since 7 years, treating in a time comorbid diagnosed or incidental.

Materials and Methods: Surgical technique consists in: pneumoperitoneum induction, with Verres, in left upper quadrant; disposition of $10 \mathrm{~mm}$ optical trocar and two $5 \mathrm{~mm}$ trocars in the left anterior axillary line; $5 \mathrm{~mm} 30$ degree optic for prothese introduction viewing; adhesiolisis (cold blade near bowel); intra and extra abdominal measurement, transparietal placement markers to sign an overlap $>4 \mathrm{~cm}$; adjustment of "teilored prothese"; currently mixed fixing (biological glue and half of the usual of titanium or reabsorbable spirals) reducing traumatism and postoperative pain $(<57 \%)$. Preoperative assessment of patient using US and eventually CT of abdominal wall.

Results: From 2003 to 2010 we have performed 203 laparoscopic treatment of abdominal wall hernias: middle lesion size 7,5 $\mathrm{cm}(2-20 \mathrm{~cm}), 77$ pt. $(37,9 \%)$ with BMI $>30,120$ female and 83 male, middle age 61,8 years old ( 24 and 83 ). 140 incisional hernias (27 recurrent), 34 umbelical, 28 epigastric and 1 Spiegel. 110 extended adhesiolisis. 18 pt. with strangulated hernia. 32 tratments ofconcomitant diseases. 3 intraoperative complications occurred during adhesiolysis: 2 bowel and 1 colonic injury laparoscopically repaired; 3 mayor postoperative complications: 1 ileal suture dehiscence, 1 massive aspiration pneumonia, 1 migrate into the bladder protack and 1 seroma . Relapse rate (F.U. > 12 months) $2,1 \%(4 / 183)$.

Conclusion: We want to underline the benefits of laparoscopic technique: less complications, less relapse, less operative time, less middle hospital stay. The result is an improvement in patient satisfaction.

\section{2 \\ Esophageal Myotomy: History of a Laparoscopic Success}

\author{
E.L. Ratto, A. Noceti, A. Rota, F. Maritato*, C. Eretta*, \\ A. Piccardo \\ S.C. Chirurgia Generale- Direttore Prof. A. Piccardo, ASL2 \\ Savonese Ospedale di Cairo Montenotte - Ospedale di \\ Savona, ${ }^{*}$ S.S. Pronto Soccorso Ospedale di Cairo \\ Montenotte - Responsabile dott. F. Maritato
}

Introduction: Currently the best therapeutic option in the management of esophageal achalasia seems to be a laparoscopic 
Heller myotomy, since it gives better and more durable results than pneumatic dilatation and intrasphincteric injection of botulinum toxin, while it is associated to a short hospital stay and a fast recovery time.

Materials and Methods: According with international literature, our equipe performs a laparoscopic Heller myotomy with fundoplication to treat esophageal achalasia. Our technique consists in: pneumoperitoneum induction with periumbelical Verres; disposition of 5 trocars crescent in superior abdomen; laparoscopic exploration; isolament of gastric fundus cutting short gastric vessels; isolament of esophagus from dyaphragm till left pulmonary vein; longitudinal esophageal myotomy from gastric fundus till middle esophagus $9 \mathrm{~cm}$ long; hiatus plastic; Nissen-Rossetti fundoplication; periesophageal dreinage.

Results: Review of personal experience from 1983 to 2010. From left thoracotomy with esophageal myotomy and BelseyMark IV fundoplication, to laparoscopic Heller myotomy with Nissen-Rossetti fundoplication, performing in the meantime laparotomic Heller myotomy with Nissen fundoplication. Middle operating time from 250 minutes to 105 minutes. Middle hospital stay from 20 days to 4 days.

Conclusion: We believe in the efficacy and the safety of laparoscopic Heller myotomy in the management of esophageal achalasia. We want to underline the importance of laparoscopy in inducing gastroenterologist and patients in accepting surgical treatment as the best therapeutic option.

\section{3 \\ Microlaparoscopic Technique in Ventral Hernias Repair
A. Noceti, E.L. Ratto, A. Rota, F. Maritato*, C. Eretta*, A. Piccardo
S.C. Chirurgia Generale- Direttore Prof. A. Piccardo, ASL2
Savonese Ospedale di Cairo Montenotte - Ospedale di
Savona, ${ }^{*}$ S.S. Pronto Soccorso Ospedale di Cairo
Montenotte - Responsabile dott. F. Maritato

Introduction: 18 years after the first laparoscopic repair of spigelian hernias and 17 years after the first series of 5 laparoscopic incisional hernias repair, the technique can be considered standard. We want to introduce a new microinvasive technique: "two trocars technique", to further reduce the traumatism.

Materials and Methods: Our technique consists in: pneumoperitoneum induction, with Verres, in left upper quadrant; disposition of 2 trocars: one $10 \mathrm{~mm}$ optic, for prothese introduction, and one $5 \mathrm{~mm}$ in the left anterior axillary line; $5 \mathrm{~mm} 30$ degree optic; intra and extra abdominal measurement transparietal placement markers to sign an overlap $>4 \mathrm{~cm}$; ; adjustment of "teilored prothese"; mixed fixing (biological glue and half of the usual of titanium or reabsorbable spirals) reducing traumatis. Preoperative assessment of patient using US and eventually CT of abdominal wall. Patients with significant peritoneal adhesions are excluded from this technique.

Results: From 2007 to 2010 we have performed 35 operations: middle lesion size $4 \mathrm{~cm}(2-6 \mathrm{~cm}), 12$ pt. (34,2\%) with BMI $>30,22$ female and 13 male, middle age of 60,5 years old (35 and
89). 20 incisional hernias ( 7 recurrent and 4 complex defect treated with double prothese), 9 umbelical, 5 epigastric and 1 Spigelian. Middle operative time: 45 minutes (25-85 min). No postoperative complications. Reduction in postoperative analgesic assumption of 57\%. Middle hospital stay 30 hours. Relapse rate (F.U. > 24 months): $0 \%$.

Conclusion: We consider this technique effective and safe. We think that its utilization could be another step towards the micro invasiveness, with great advantage for the patients.

\section{4}

\section{Laparoscopic Appendectomy Benefits}

E.L. Ratto, A. Noceti, A. Rota, F. Maritato*, C. Eretta*, A. Piccardo

S.C. Chirurgia Generale- Direttore Prof. A. Piccardo, ASL2

Savonese Ospedale di Cairo Montenotte - Ospedale di

Savona, ${ }^{*}$ S.S. Pronto Soccorso Ospedale di Cairo

Montenotte - Responsabile dott. F. Maritato

Introduction: Since 1992, according with EBM approach, several studies have underlined the diagnostic value, clinical outcomes and laparoscopic appendectomy benefits (LA). We want to confirm these analises comparing our experience with international literature.

Materials and Methods: Diagnostic value Laparoscopic surgery is, first of all, a diagnostic technique. EBM: diagnostic accuracy of 95-99\%; diagnosis of occasional disease in $20-30 \%$ of LA.

Clinical Outcomes: The biggest advantage lies in complicated appendicitis: better view, washing and drainage of the abdominal cavity, resulting in better therapeutic strategy (possibility of using endoGIA). Resulting: low rate of postoperative complications: intrabdominal infections, wound infections; shorter hospital stay; less postoperative pain; quicker recovery ensuing.

Benefits: Aesthetic result (above all with micro-appendectomy)

Economical advantage: total cost (direct and indirect) LA 11577 \$ versus OA 13956 \$ (Laparoscopic Appendectomy Interest Group - Mayo Clinic and Mayo Foundation 2001)

Results: Since 2002, after adequate training, our team prefer laparoscopic approach. On 886 appendectomies: 531 (59,93\%) were LA and $355(40,06 \%)$ OA. Currently, LA is the treatment of choice. In such cases we perform "micro-laparoscopic technique": three $5 \mathrm{~mm}$ trocars; double endoloop on appendix base or purse string suture. In complicated appendectomy we use three trocars (one $10 \mathrm{~mm}$ and two $5 \mathrm{~mm}$ ) and endoGIA.

Conclusion: LA is better than OA from a diagnostic, therapeutic and clinical outcome. The result of micro invsiveness is a low rate of complications and a quicker recovery. Also the costs of management seem to be in favour of LA. 


\section{5}

\section{Risk Factors for Single-Stage Surgery for Synchronous Diseases: A Retrospective Analysis}

\author{
C. Chiappa, S. Rausei, F. Rovera, L. Boni, P. Castano, \\ G. Dionigi, R. Dionigi \\ Department of Surgery, University of Insubria, Varese
}

Introduction: To identify morbidity risk factors in patients with synchronous diseases underwent single-stage surgery.

Methods: We considered the data of 328 patients each with multiple, elective, synchronous surgical problems treated by a single-stage operation ( $2 \%$ of the overall surgical workload). By univariate and multivariate analyses we evaluated many patient-, disease- or treatment-related variables respect to postoperative mortality, morbidity, and hospital stay.

Results: Two combined procedures were synchronously performed in 283 patients (86\%), 3 combined procedures in 45 patients (14\%). Postoperative mortality and morbidity rates (overall $3 \%$ and 24\%, respectively) and hospital stay length (overall 11.1 days +8.5 ) were comparable to historical series after data control for relevance of surgical problems. The occurrence of a surgical oncology procedure resulted the most important independent risk factor for postoperative mortality, morbidity, and hospital stay. The other independent risk factors were the operative time $>150$ min and the first procedure treating an abdominal problem with a high surgical severity score.

Conclusion: Single-stage surgery for the treatment of synchronous surgical problems seems safe like the conventional procedures. The understanding of risk factors for this surgical approach will be useful in order to further improve the patients selection.

\section{6}

Open Abdomen for Management of Severe Peritonitis: Retrospective Interim Analysis for a Data Collection Based on Twenty-Year Experience

F. Rossi, G. David, S. Rausei, C. Valerii, S. Binda, G. Selmo,

A. Del Bosco, G. Dionigi, G. Carcano, R. Dionigi

Department of Surgery, University of Insubria, Varese

Introduction: In order to predict the appropriateness of indications, timing of definitive closure and patient outcome of open abdomen (OA) approach, well-designed retrospective also studies could be useful. We present an interim analysis for optimizing the data collection based on our twenty-year experience.

Methods: For data collection we designed a retrospective database including clinical details, surgical findings and parameter of intensive care unit. For interim analysis we selected a random sample for $20 \%$ of the population. We considered only data available for all patients, together with well-known prognostic score systems, with respect to outcome.
Results: 22 patients were considered. Twenty-one patients (95\%) underwent OA for severe peritonitis. Five patients died. By nonparametric tests we only observed a statistical significant association between APACHEII score at admission and outcome $(\mathrm{p}=0.006)$. The number of revisions and the length of OA were not associated with outcome.

Discussion: The mortality rate in this interim analysis (23\%) was lower when compared to historical series. According to the suggestions of these preliminary results, we must complete the analysis on our total population. At present, we suppose that the patient outcome is affected by clinical status at admission more than by risks associated to $\mathrm{OA}$.

\section{7 \\ Single Port Laparoscopic Colectomy: Two Clinical Cases Report}

\author{
M. Rigamonti, G. Perigli, N. Battisti, E. Qirici, F. Cianchi \\ Department of General Surgery, University of Florence, \\ Italy
}

Introduction: Single Incision Laparoscopic Surgery (SILS) represents a recent innovation in mini-invasive laparoscopic surgery. Surgical procedures are performed through a single incision preferentially made on the umbilical scar, using a multi-lumen port (Single Port) and flexible curved instruments. This technique avoids the need for triangulation which is instead required in conventional laparoscopy. To our knowledge, there are only few published studies describing single incision left emicolectomy.

Materials and Methods: Between February and March 2010, two laparoscopic single incision left colectomies through a 3 $\mathrm{cm}$ umbilical incision were performed in two female patients ( 31 and 40 year old) with diagnosis of sigmoid in situ colon adenocarcinoma previously treated by not radical endoscopic polipectomy. We used a SILS Port (Covidien, MA, USA) along with a $5 \mathrm{~mm}$ video-laparoscope, and curved rotating and articulated instruments.

Results: Both surgical procedures were completed in less than 150 minutes. Blood loss was minimal. Patients had no intraoperative and post-operative complications and lengths of hospital stays were 4 and 5 days. Pathological examination did not show residual tumor tissue in both colonic specimens and harvested limph nodes. Cosmetic result was optimal after seven days.

Conclusion: SILS can be performed with good surgical and oncological results also in selected patients with colorectal cancer. 


\section{8 \\ Early and Late Complications in their Treatment of Haemorroidal Disease with PPH vs Milligan-Morgan}

A. Rizzuto, F. Ferrari, A. Carpino, P. Pafundi, R. Colace, G. Vescio, R. Romano, G. Sammarco, R. Sacco

Background: The aim of this study was to assess the early and late complications and the recurrence of haemorrhoidectomy according to Milligan-Morgan (laser variant surgical fibre)vs haemorrhoidopexy with PPH-stapler in patients with circumferential prolapse classified P4-E4 according to PATE 2000 classification.

Methods: From January 2001 until December 2007160 randomizied patients with Haemorrhoidal disease classified P4-E4 were included. Patients were split-up in two groups. GROUP A (MM laser fibre) 80 pateints (50 Male,30 Female;median age 39 years,range 23-57)

GROUP B (PPH-Stapler) 80 patients (58 Male, 22 Female; median age 40 years, range 23-60).

Range Pain was evaluated with verbal numeric scale. Statistical analysis was performed with a SPSS for windows. Pain score and complications were respectively assessed with test T-student and Chi -squared.

Results: V.N.S. was inferior in th PPH-group,with $\mathrm{p}<0.0017$ (significative result for $\mathrm{p}<0.05$ until 72 hours) and $\mathrm{p}<0.0001$ (significative for $\mathrm{p}<0.01$ until 7 days). Early Complications: thrombosis and urinary retention were higher in the first group than in the second one, respectively ( 6 cases $M-M$ vs 1 in $\mathrm{PPH})(\mathrm{p}=0.05)(13$ vs 5) $0,01<\mathrm{p}<0,005$ (chi square 4,006 )

Late complications: Occasional bleeding occurs in 13.5\%(MM group) vs $10 \%$ (PPH-group), $\mathrm{p}>0.1$

Defecatory urgency $2,5 \% \mathrm{MM}$ vs $5 \% \mathrm{PPH}, 0.05<\mathrm{p}<0.1$. Soiling $19.75 \% \mathrm{MM}$ vs0 \% $\mathrm{p}<0.001$. Recurrence rate $5 \% \mathrm{PPH}$ vs $0 \%$ $\mathrm{p}<0.05$. Residual disease :MM 7,5\%vs0\% $\mathrm{p}<0.01$.

Conclusions: PPH stapler procedure is a feasible and reproductable technique for the treatment of haemorrhoidal disease with clear indications in circumferential prolapse P4-E4.

89

\section{Single- Incision Laparoscopic Cholecystectomy:Is Possible to Find Pre- operative Indications?}

\section{A. Rizzuto, F.U. Zittel, G. Vescio, G. Sammarco, R. Sacco \\ Universita' Degli Studi Magna Graecia Catanzaro, Ev. Hochstift Krankenhaus Worms, Germany}

Background: In recent years, natural orifice transluminal surgery (NOTES) as been offered as the next generation of minimally invasive surgery with regard to reducing post-operative pain decreasing complications and improving cosmesis. The indications for this surgical approach are until now not clear.
Methods: Between october 2009 and april 2010, 80 patients underwent colecystectomy via SILS, the umbelicus was the access point to entry to the abdomen for all patients. The patient's history and clinical assessment were verified by ultrasonography with particular attention to the cholecyst's anatomic variations and Calot's triangle.

BMI $>30$, acute clinical presentation and previous abdominal surgery weren't exclusions criteries.

Results: Of 80 cholecystectomies performed with SILS, just 5 needed the insertion of $5 \mathrm{~mm}$ trocars. The conversions in conventional laparoscopic cholecystectomy were associated with cholecyst's anatomic variations or of cystic artery, 50\% previously identificated by ultrasonography.

Any correlation between BMI,previous operation and acute presentation was observed. Post -operative paine was for all the patient $<3$ (scale 0 -10) 1 postoperative blood and 1 umbelical hernia were observed.

Conclusions: The use of SILS for cholecystectomy is safe and feasible also in patients with higher BMI, previous operations and acute presentation.

The rate of conversion to conventional laparoscopic approach seems correlated to particular anatomy but randomized studies are still necessary.

\section{0 \\ Current Role of "Hanging Moneouvre" for Major Hepatectomy}

E. Rosso, F. Panaro, E. Marzano, I. Lucescu, P. Patrick, P. Bachellier

Centre de Chirurgie Viscérale et de Transplantation, Hôpitaux Universitaires de Strasbourg, Université Louis Pasteur, Avenue Molière, 67098 Strasbourg, France

Introduction: Mobilisation of the right liver, in case of huge right liver lobe tumour, may expose to major bleeding of tumoral rupture. The Hanging manoeuvre may contribute to increase the safety and long term results of such procedure.

Materials and Methods: We present the case of a 44 year old patient with a single liver tumour invading almost all the right liver lobe as well as the right diaphragm. After dissection of the cavo-hepatic veins confluence and of the inferior vena cava; the "hanging manoeuvre" was performed. The right hepatic artery and the right portal vein were controlled and sectioned. A right hepatectomy was performed assisted by the traction on the drain used for the hanging manoeuvre. A larger patch of diaphragm was resected en-block with the liver.

Results: During liver transection no pedicle clamping was performed. Blood lost was minimal and no transfusion was required. No tumoral rupture happened. Postoperative period was uneventful. Resection margins were free.

Conclusion: The present case underlined that the "Hanging moneouvre" contributes to increase the safety of right hepatectomy in casse of huge right liver lobe tumour. 


\section{1}

\section{Evolution of Indication to Surgery for Benign Liver Tumors (BLT) in a 29-year Single Center Experience}

\author{
E. Ponte, G.L. Grazi, F. Sacchetti, P. Di Gioia, G. Vetrone, \\ M. Del Gaudio, A.D. Pinna \\ Liver and Multiorgan Transplant Unit, Policlinico S. Orsola- \\ Malpighi, University of Bologna, Italy
}

Introduction: Despite the great number of studies in literature, management of benign hepatic tumors is still a matter of discussion. Accurate diagnosis can now be achieved in a large percentage of patients and is mandatory for the decision of whether to apply surgery or observation.

Materials and Methods: Our series of 261 patients was divided into two groups, the first ranging from 1980 to 1995 (129 patients) and the second from 1996 to 2009 (132 patients). This study was directed to evaluate the possible modifications in the cause of discovery and indication to surgery between the two periods for BLT. The population was composed by: 104 hemangioma, 67 cysts, 57 focal nodular hyperplasia and 33 adenoma.

Results: Evolution of imaging techniques justifies the increase of incidental findings (from $40.6 \%$ to $63.3 \%, \mathrm{p}=0.04$ ) and allow to diagnose lesions before the onset of symptoms (from $59.4 \%$ to $36.7 \%, \mathrm{p}=0.001$ ). Surgical resections performed for symptomatic lesions are decreased $(54.3 \%$ vs $25.9 \%, \mathrm{p}=0.001)$. Diagnostic dilemma, especially for adenomas and focal nodular hyperplasia, is one of the principal cause of resection $(23.9 \%$ and $27.9 \%, p=n s)$. Due to the lack of prospective and controlled studies with a long follow up regarding adenomas, in our series these lesions are still resected with formal indication. The volumetric increment is a frequent cause of resection because of the strict follow up performed in all patients.

Conclusion: These recent findings modified indications to surgery for BLT but decision to apply surgery or observation is still argument of discussion.

\section{2 \\ Efficacy and Safety of Calcineurin-inhibitor- free De-novo Immunosuppression with Everolimus After Liver Transplantation}

F. Sacchetti, G.L. Grazi, E. Ponte, P. Di Gioia, G. Vetrone, M. Del Gaudio, A.D. Pinna

Liver and Multiorgan Transplant Unit, Policlinico S.OrsolaMalpighi, University of Bologna, Italy

\section{2nd Classified - SPIGC Award Session, General Surgery}

Introduction: Immunosuppression with calcineurin inhibitors (CNI) increases the risk of renal dysfunction after orthotopic liver transplantation (OLT). Based on this clinical data this study protocol was designed to assess the efficacy and safety of calcineurin-inhibitor-free de-novo immunosuppression with mTOR inhibitor, Everolimus, after liver transplantation for HCV-related cirrhosis.

Materials and Methods: A prospective therapeutic study in a total of 45 liver transplant patients was designed to assess the safety and efficacy of de-novo CNI-free immunosuppression with basiliximab, prednisolone and Everolimus in comparison with basiliximab, prednisolone and Tacrolimus. The primary endpoint were the rate of rejections and the rate of HCV infection recurrence. Secondary endpoints were the mortality up to one year after OLT, liver allograft function (assessed by measurement of AST, ALT, total bilirubin, AP, GGT), kidney function (assessed by measurement of serum creatinine and creatinine clearance) and overall incidence of adverse events (infections rate, neurotoxicity, nephrotoxicity, incidence of de-novo-diabetes, hyperlipidemia and edema).

Results: Preliminary data show the substantial feasibility of this therapeutical approach for what concerns both the primary and secondary endpoints. Rejection rate was comparable (9.1\% vs $0 \%, \mathrm{p}=\mathrm{ns}$ ) as well as HCV recurrence ( $45.5 \%$ vs $43.5, \mathrm{p}=\mathrm{ns})$. The most important collateral effect in the Everolimus study group was the onset of lower limbs edema ( $45.5 \%$ vs $8.7 \%$, $\mathrm{p}=0.005)$, whereas tacrolimus treated patients developed more frequently de-novodiabetes $(26.1 \%$ vs $4.5 \%, \mathrm{p}=\mathrm{ns})$.

Discussion: If these data corroborate safety of de-novo CNI-free immunosuppressive regimen this should be confirmed in other randomized, prospective, controlled double-blinded clinical trial.

\section{3}

\section{Complicated Management of latrogenic Esophageal Injury}

T. Saibene, M. Valmasoni, M. Costantini, E. Finotti, R. Salvador, E. Ancona

Clinica Chirurgica Generale Prima -padova-

We describe the complicate mangement of an oesophageal injury due to emergency treatment of gastrointestinal bleeding.

The Patient, 46 years old female, with history of alcoholic cirrhosis and many episodes of anaemia because of splenic artery pseudo aneurysm, comes to our attention because of iatrogenic oesophageal rupture.

In January 2009, the patient was admitted to another hospital because of serious anaemia $(\mathrm{Hb} 3.9 \mathrm{mg} / \mathrm{dL})$ and she was treated non-operatively with blood transfusions.

Suspecting active gastric bleeding, Blakemore tube was placed and gastric fundus resection was performed (February 2009). A post procedure thoracic CT scan showed oesophageal rupture and splenic artery pseudo aneurysm that required embolization and bipolar oesophageal exclusion with cardia stapling, digiunostomy and simultaneous oesophageal fistula treatment with nasogastric tube suction (February 2009). Because of hemorrhagic recurrence and radiological diagnosis (abdomen CT scan) of splenic infarction, the patient underwent splenectomy and bleeding gastric ulcers repair (March 2009). Another bleed required arterial embolization with 10 coils (April 2009). Once the patient was stable and the oesophageal reepithelization was confirmed, the patient finally 
underwent endoscopic recanalization of the oesophageal lumen and cervical esophagostomy closure (July 2009). Acute treatment of oesophageal bleeding requires a careful use of devices such as Blakemore tube.

\section{4}

\section{Current Trends in Polytrauma Management, Revision of Algorithms Operational in the Trauma Center of Cesena, Italy}

\section{P. Ruscelli, N. Fabbri, L. Bergossi, D. Drudi, S. Santella, R. Bertelli, E. Faccani, B. Turri, R. Cavallini, F. Buccoliero*}

*U.O. di Chirurgia d'Urgenza e del Trauma, Dipartimento di Emergenza, Trauma Center, Presidio Ospedaliero "BufaliniMarconi-Angioloni", Azienda Sanitaria di Cesena

Introduction: The purpose of this paper is to check what impact the progressive implementation and review of our algorithms has had on predefined indicators of results and utilization of diagnostic and therapeutic resources. Also for the purpose of comparing the results obtained in a subgroup of patients treated in the Trauma Center of Cesena, with those obtained in a group homogeneous stored in the Regional Major Trauma Registry.

Materials and Methods: We analyzed a population of 21,704 patients. They were divided into 3 groups based on hemodynamic response after primary assessment. Group A included patients that were hemodynamically stable; Group B included patients that were hemodynamically stabilized; Group C included patients that were hemodynamically unstable. Each group of patients was treated according to precise diagnostic and therapeutic protocols.

Results: The overall hospital mortality was $2.4 \%$. Mortality at discharge from intensive care was $11.6 \%$ while at discharge from the Emergency Surgery was $0.2 \%$. The total average hospital stay was 10.1 days. $79.4 \%$ of the patients were discharged home. Patients undergoing diagnostic level II with multislice CT were $19.3 \%$; those undergoing CT of the chest and / or abdomen were $5 \%$. A total $0.8 \%$ of patients underwent angiography, and $0.2 \%$ underwent embolization.

Conclusions: We believe that these protocols can provide a valuable and effective aid for those involved in the care of trauma victims, allowing them to always be able to quickly decide what to do, when to do, how to do, and where to do what needs to be done.
95

NOM of Hepatic and Spleen Injuries and Impact on the Follow Up: Experience in the Trauma Center of Cesena
P. Ruscelli, N. Fabbri, L. Bergossi, D. Drudi, S. Santella, R. Bertelli, E. Faccani, B. Turri, R. Cavallini, F. Buccoliero*
*U.O. di Chirurgia d'Urgenza e del Trauma Dipartimento di Emergenza - Trauma Center - Presidio Ospedaliero
"Bufalini-Marconi-Angioloni" - Azienda Sanitaria di Cesena

Introduction: The introduction of NOM as well as Damage Control for hepatic and/or splenic injuries rappresents the most important change in the management of trauma patients since 2001 in the Trauma Center of Cesena. The aim of this study is to illustrate the recently reviewed diagnostic and therapeutic protocols for the in hospital management and the follow up of the NOM patients now operational in our Trauma Center and to analyze the impact of the splenic preservation on the return to the normal activities and life style of these patients after the discharge from the Hospital.

Material and Methods: The Trauma Service has been the organism inside which the revision of these algorithms has been developed. Crucial points such as inclusion and esclusion criteria for NOM, relastionship between hemodinamic pattern of the patient and grade of the injury, role of CEUS in the monitoring and follow up of these patients, timing of return to normal activities and sports have been analyzed.

A cohort of 91 patients treated successfully with NOM for blunt splenic or hepatic injuries has been evaluated.

Results: No patients have been readmitted to the hospital after the discharge for the same illness. CEUS has demonstrated that the complete healing of the hepatic injuries has been achieved within a period of time shorter than the spleen lesions.

Conclusions: The impact of NOM on the follow up of these trauma patients is quite different between those with hepatic injuries and spleen injuries.

\section{6 \\ PACS: A Modified Laparostomy Technique Results After Two Years of Experience in the Cesena Trauma Center \\ P. Ruscelli, N. Fabbri, L. Bergossi, D. Drudi, S. Santella, R. Bertelli, E. Faccani, B. Turri, R. Cavallini, FBuccoliero* \\ $\left.{ }^{*}\right)$ U.O. di Chirurgia d'Urgenza e del Trauma Dipartimento di Emergenza - Trauma Center - Presidio Ospedaliero \\ "Bufalini-Marconi-Angioloni" - Azienda Sanitaria di Cesena}

Introduction: In the last few years laparostomy has become an even more useful option for the surgeon due to the development of the experience in the critical abdominal trauma and in the severe surgical urgencies as PANE and peritonitis with the introduction of Damage Control as surgical strategy to prevent the abdominal compartimental syndrom (ACS). After a laparostomy 
the surgeon needs to provide a delayed fascial closure (DFC) to achieve the best outcome for the patient. The aim of this paper is to illustrate the experience and the results after the introduction of a modified laparostomy technique in our surgical activity.

Materials and Methods: Protection of intestinal content, aspiration under sub-athmospheric pressure, control in quality and quantity of the drainage and skin temporary closure, rappresent the four principles of the PACS tecnique. In order to evaluate the rate of delayed fascial closure and the rate of ACS onset, we have retrospectively studied 31 patients over a period of 2 years treated with open abdomen with an amount of 36 PACS performed. Routinely IAP measurament was also performed.

Results: ACS was developed in no patients. IAP was measured on average at 19 . DFC was achieved in 33 PACS of 36 $(91,7 \%)$ after 5 days on average (20 hours as minimum; 20 days as maximum). Aspected mortality (SAPSII) in ICU for these patient was $76,5 \%$ on average. On 31 patients, $20(64,5 \%)$ died and 11 $(35,5 \%)$ survived.

Conclusion: PACS seems to be an effective, easy and low cost technique for the management of open abdomen.

\section{7 \\ Liver Resection for Early Hepatocellular Carcinoma $(\leq \mathbf{3} \mathrm{cm})$. A Multicentric Experience}

\section{G. Sarno, G. Ranucci, F. Ardito, F. Giuliante, G. Nuzzo}

1st Classified - SPIGC Award Session, General Surgery

Background/Purpose: Recently the operative risk of liver resection (LR) has significantly been reduced. Early hepatocellular carcinoma (EHCC, $\leq 3 \mathrm{~cm}$ ) is more frequently diagnosed, mainly due to strict surveillance strategies in cirrhotic patients. LR remains the first line treatment. Hereby we report the experience with LR for EHCC in a multicentric study.

Methods: 588 consecutive patients who underwent LR for EHCC were retrospectively analyzed. Patients and tumour characteristics, postoperative morbidity and mortality, and 5- and 10 -year overall and disease-free survival (Kaplan-Meier) were evaluated.

Results: Male/female ratio was 5/1. Median age was 66 years (range 22-85). HCV-related hepatitis was most commonly present (63\%). 547 patients (93.0\%) were Child-Pugh class A, 454 patients (77.2\%) had a single tumour, 229 patients (38.9\%) had tumour size $\leq 2 \mathrm{~cm}$.

Postoperative morbidity was $36 \%$, major morbidity (Clavien classification) was $7 \%$. There were $11(1.9 \%)$ perioperative deaths. After a mean follow-up of 46.7 months the 5- and 10-year survival rates were $52.6 \%$ and $20.2 \%$, respectively, with 5- and 10 -year disease-free survival of $32.6 \%$ and $21.8 \%$, respectively. Patients were stratified into 3 groups according to the presence of pathologic factors (tumour size $>2 \mathrm{~cm}$, microvascular invasion, multifocal tumour). Patients with $\geq 2$ factors had significantly lower overall survival at univariate analysis. Presence of satellite nodules was an independent poor prognostic factors for both overall and diseasefree survival at multivariate analysis.

Conclusions: LR is safe and effective in patients having EHCC and well preserved liver function. Pathologic factors are valid tools to assess long-term prognosis even in patients with EHCC.

\section{8}

Evaluation of a Breast Cancer Nomogram for Predicting the Likelihood of Additional Nodal Metastases in Patients with a Positive Sentinel Node Biopsy

\author{
S. Scomersi ${ }^{1}$, L. Torelli ${ }^{2}$, F. Zanconati ${ }^{3}$, M. Bortul ${ }^{1}$ \\ ${ }^{1}$ Dipartimento di Scienze Chirurgiche Generali, \\ Anestesiologiche e Medicina Intensiva, Istituto di Clinica \\ Chirurgica Generale e Terapia Chirurgica, \\ ${ }^{2}$ Dipartimento di Matematica ed Informatica, \\ ${ }^{3}$ Dipartimento di Scienze Cliniche, Morfologiche e \\ Tecnologiche
}

Introduction: Sentinel node (SLN) biopsy has been demonstrated to be accurate for assessing regional lymph node involvement in breast cancer patients. $40-70 \%$ of patients with positive SLNs are found to have no other nodal metastases at further complete axillary dissection (CAD). A nomogram has been developed at Memorial Sloan Kettering Cancer Centre (MSKCC) to predict the likelihhod of non-sentinel node (NSLN) metastases after a positive SLN biopsy. The purpose of this study is to evaluate the nomogram.

Materials and Methods: From a retrospective database of 276 breast cancer patients treated at our institution we evaluated 62 positive SLN biopsies. We analysed the correlation between positive NSLN and tumor characteristics (Test F Fisher and Chi Square). We also assessed the accuracy of MSKCC nomogram to predict NSLN metastases by the Receiver Operating Characteristic (ROC) curve.

Results: Presence of extranodal extension in the $\mathrm{SN}(\mathrm{p}=0.013)$ of patients harbouring a macrometastasis $(\mathrm{p}=0.03)$ was found to be associated with NSLN metastases, while other features (age, lymphovascular invasion, hystology, grading, number of positive $\mathrm{SN}$, biological characteristics) were not. The accuracy of MSKCC nomogram as measured by the area under the ROC curve, was 0.67 . With a $10 \%$ cut-off value the nomogram showed $95 \%$ sensitivity and $14 \%$ specificity. We revised the nomogram by incorporating the presence of extranodal extension and we obtained a new test with improved specifity (84\%).

Conclusion: The modified predictive model is a useful tool in predicting the likelihood of NSLN and may help decision regarding the need of completion axillary lymph node dissection. 


\section{9 \\ The "Ghost-Ileostomy": An Easy and Effective Technique}

\author{
A. Marrosu, F. Serventi, M.A. Sassu, C. Torre, I.P. Pisano, \\ F. Attene, F. Scognamillo, E. Trignano, M. Trignano
}

Institute of Surgical Pathology, University of Sassari, Italy

Introduction: Anastomotic breakdown is unpredictable and the decision whether to fashion a protective stoma or not is based on the surgeon's experience. Consequently many patients will receive an unnecessary stoma as they will not have an anastomotic breakdown. The aim of fashioning a "Ghost Ileostomy" (G.I.) is to avoid these unnecessary stomas. This study aims at evaluating efficacy and safeness of the "G.I.".

Methods: From 2008 to 2009 we fashioned 20 "G.I." in patients who underwent ultra-low anterior rectal resection. Before abdominal closure the ileum was under passed by a vessel loop about $20 \mathrm{~cm}$ from the ileocecal valve. The two extremities of the vessel loop were exteriorized in the right fossa iliaca and fixed to the skin. In case of anastomotic break-down an excluding ileostomy was fashioned using the guide of the "G.I.".

Results: 18 patients didn't present signs and symptoms of anastomotic breakdown and the vessel-loop was removed between the $10^{\text {th }}$ and $12^{\text {th }}$ postoperative day without any complications related to the presence or removal of the vessel-loop. In two cases we performed an excluding loop-ileostomy using the prefashioned "G.I." in $8^{\text {th }}$ and $9^{\text {th }}$ postoperative day. Both patients had a regular postoperative course.

Conclusion: The "Ghost Ileostomy" is a safe and easy method and permits to fashion a stoma only when necessary avoiding many needless stomas.

\section{0}

\section{Altemeier Rectosigmoidectomy: A Procedure Worth Considering}

\section{F. Serventi, A. Marrosu, S. Denti, M.A. Sassu, P. Paliogiannis, C. Torre, C. Pala, M. Trignano \\ Surgical Pathology Institute, University of Sassari, Italy}

Introduction: Several surgical techniques have been proposed for treatment of complete rectal prolapse. Mainly they can be distinguished in trans-abdominal and transperineal approaches. Former abdominal surgery can preclude a transabdominal approach. We report a case of complete rectal prolapse, treated by transperineal rectosigmoidectomy, in a patient with history of pelvic surgery.

Case Report: A 61 years old female patient with soiling was admitted for a $7 \mathrm{~cm}$ long, complete, rectal prolapse. Ileo-cystocolpo-defecography showed insufficient increase of the ano-rectal angle and a functional enterocele. At anorectal manometry the basal tone was decreased with good squeeze pressure. Considering that the patient had former pelvic surgery for renal TBC with bladder resection and bladder-ileo-plasty we decided for a trans- perineal rectosigmoidectomy. Postoperative stay was regular and she experienced immediate clinical remission and anorectal manometry two months after surgery demonstrated objective improvement.

Discussion and Conclusions: Most authors consider the trans-abdominal approach to be of first choice, while transperineal techniques are suitable only for patients with contraindications for an abdominal approach. Recent studies showed that the higher recurrence rate of perineal techniques could be secondary to a selection bias as these approaches are mostly used in older and frail patients. In this few perineal techniques could be considered to be of first choice even in young patients.

\section{1 \\ Suturless - Lightweight Hernioplasty vs Traditional Lichtenstein Repair. One Year Results on Chronic Postoperative Pain

\author{
C. Stabilini, R. Fornaro, F. Lazzara, F. Mandolfino, \\ M. Imperatore, E. Gianetta \\ Dipartimento di Chirurgia dell'Università di Genova
}

Background: The aim of the study is to assess whether a partially absorbable monofilament mesh fixed with fibrin sealant could influence postoperative pain and time to return to normal activity in comparison to traditional Lichtenstein hernioplasty.

Methods: The study randomized patients undergoing primary inguinal hernia repair into groups according to mesh type and fixation method: group 1 (lightweight mesh - fibrin sealant) and group 2 (heavyweight mesh - suture). Follow-up on discharge and at 6 and 12 months evaluated the incidence of early and late complications, recurrence rate, postoperative pain and return to physical activity.

Results: A total of 162 patients were randomized (81 sutureless group vs 81 traditional group). Postoperatively no death or major general complications were recorded. Local specific complications (hematomas and sieromas) were equally distributed in both groups (2 $\mathrm{s}$ in group 1 and 1 in group 2). Group 1 reported shorter duration of analgesic use ( $1 \pm 1.3$ vs $2.1 \pm 1.7$ days; p.0029). Time to return to normal activity didn't differ among groups (3.3 vs 4.5 days). Two recurrence were recorded in group 1 at 6 months (pNS). At 6 months postoperative pain was not different $(1 \mathrm{~s}$ in group 1 and 2 in group 2; pNS). Postoperative pain disappeared in both group at 12 months.

Conclusion: Use of partially absorbable mesh reduced postoperative pain in the short term. No difference in pain or recurrence rates were observed at 12 months. 
102

\section{Wound Care for Post Traumatic Complex Inferior Limbs Injuries: Use of NPWT, Advanced Medications and Amino Acids}

\section{B. Tarantino, L. Valesini, L. Centonze, M. Assenza,}

C. Modini

Emergency Dept., Policlinico Umberto I, Sapienza

University of Rome, Rome, Italy

Introduction: Post-traumatic inferior limbs injuries represent a challenging problem for surgeons, both during the initial emergency treatment, and for their reconstructive management. The reconstructive treatment takes advantage in isolated or arranged way of advanced medications, NPWT, necrosectomy, wet medications, PRP gel and surgery. Actually essential amino acid use finds recent application and it's essential in the constitution of the muscular woven one.

Materials and Methods: We introduce some cases of posttraumatic inferior limbs injuries exemplified on the use and effectiveness in terms of reduced number of amputations and control of the process of healing, of treatments arrange to you with NPTW, PRP gel, and amino acids per os.

Results: The study evidences a real control of the times of definitive tegument turnover for patients who received a nutritional support; this kind of treatment allows the control of eventual septic conditions and MOF. The study examines therefore through the progression of the lesions, the incidence of the localized and systemic infections and the quality of the tissue regeneration.

Conclusions: This study defines how this approach determines one reduction of the amputations in case of complex injuries of the limbs. We have dealt patients with an outline defined by the debridement, NPTW, PRP gel and a nutritional support with essential amino acids.

103

WITHDRAWN:

Chirurgia Endolaringea Con Laser $\mathrm{CO}_{2}$ :nostra Esperienza

This abstract is withdrawn.
104

\section{Comparison Among Three Different Methods of Mesh Fixation in Patients Undergoing Plug and Mesh Technique for Primary Inguinal Hernia Repair: A Prospective Randomised Controlled Study}

\author{
M.F. Valentini, G. Lissidini, A. Gurrado, E. Poli, L. Ruggiero, \\ G. Piccinni, M. Testini
}

Introduction: Aim of this study was to compare the shortterm and long-term outcomes, operation time, and time of return to work of patients who underwent plug and mesh procedure for open groin hernia repair using suture $(S)$, human fibrin glue (HFG) or N-butyl-2-cyanacrylate (C), respectively for mesh fixation.

Material and Methods: From January 2003 to December 2007, 167 inguinal hernias (11 bilateral) who undergoing plug and mesh procedure, were randomly assigned to receive $S(n=59)$, HFG $(n=52)$ or $C(n=56)$ for mesh fixation.

Results: Overall morbidity rate was $38.9 \%$ in $\mathrm{S}$ group, $9.6 \%$ in HFG group and $10.7 \%$ in C group. Overall short-term morbidity was significantly higher in S group $(27.1 \%)$ than in the HFG (9.6\%) or C (8.9\%) groups, but there was no significant difference between the HFG and C groups. There was no significant difference among the groups in terms of mean postoperative stay or time to return to work. Long-term morbidity was significantly higher in the $S$ group. There was no recurrence in any of the groups. Foreign body reaction was referred in $8.4 \%$ of cases of $S$ group and in one C group patient. 
Conclusion: The use of human fibrin glue or N-butyl-2cyanoacrylate is better tolerated than sutures in the plug and mesh technique for inguinal hernia repair, in terms of immediate results and both show a better trend in long-term data.

105

Damage Control for Severe Open Fractures in the Limb: Wound Management Using V.A.C. Therapy (Vacuum Assisted Closure) and PRP gel (Platelet-Rich Plasma Gel)

\author{
L. Valesini, V. Belardi, F. Velluti, V. Usai, C. Modini
}

Summary: The wounds of lower extremity associated with high energy musculoskeletal trauma is an emergent social problem and such injuries are mainly of II and III stage of Gustilo's classification, these complex wounds often involve massive loss of soft tissue and bone.

Nowadays the use of modern techniques determined a further dramatic reduction in the infection rates and above all, in the risk of limb amputation.

The most important techniques include:

- V.A.C. therapy (vacuum assisted closure)

- PRP gel (platelet-rich plasma gel)

- Skin graft

The combined use of NPWT/ROCF and PRP gel may be an optimal management of wounds associated with high energy musculoskeletal trauma of lower extremity.

We treated a serie cases with high energy acute trauma through the combined and innovative use of advanced dressings. We report our experience to treatment of complex wounds of the limbs.

\section{6}

\section{Intrathoracic Eggshell Calcification in a Patient with Thyroid Goiter}

\section{A. Venturoni, G. Imbriglio, G. Di Giacopo, B. De Berardis \\ Department of Oncology, Departmental Simple Operative Unity of Oncologic Surgery; "G. Mazzini" Hospital - \\ Teramo}

Introduction: The eggshell calcification is one of the possible patterns of intrathyroidal calcifications; it is often associated with an intrathoracic goiter which can be classified as either primary or secondary.

Methods and Materials: We will illustrate the case of a male patient aged 71 with an history of open pneumonic and lymphatic tuberculosis treated with drugs, a previous hystory of alcoholism and chronic hepatosis. From an anamnestic point of view, the patiend did not present respiratory or compression disorders. Fortuitously, a thorax x-ray revealed an immersed neoformation that had invaded mostly the right side of mediastinum which pre- sented a coarse roundish opacity with a calcific density and a left impression on the trachea. CT revealed a not homogeneous glandular structure and the presence of a coarse calcific nodular lesion; the trachea had a slightly reduced caliber and was displaced on the left. The patient underwent stomatomy and sternotomy with the exeresis of the left superior thyroid lobus and of the mediastinal mass which had completely replaced the right lobus.

Results: The patient did not develop any complications and was discharged at home on the fourth day after surgery. The tissue of the left thyroid lobus was the seat of nodular colloid-cyst hyperplasia and the mediastinal mass was mostly composed of a diffusely calcified amorphic and necrotic material with minimal peripheral debris of thyroid tissue.

Conclusion: In general, eggshell calcifications are benign and not necessarily symptomatic. In order to have a final histological and differential diagnosis with mediastinal masses of different nature, a surgical exeresis is mandatory.

\section{7 \\ Enlogation of the Right Renal Vein for Cadaveric Kidney Transplantation: Our Experience with a New Stapler Technique}

\author{
F. Villa, M. Tozzi, G. Carcano
}

Objective: The anastomosis of a short right renal vein graft is associated with different difficulties especially when the recipient has deep iliac vessels or severe obesity. We present our experience with a novel technique of enlogation using a vascular stapler.

Methods: Between January 2009 and October 2009, 35 cadaveric kidney transplants have been performed, 26(74.2\%) of them using a right kidney. In all cases, the right kidney renal vein was elongated with a patch of inferior vena cava by suturing it above and below the origin of the left renal vein using a vascular stapler. The group of kidneys prepared using stapler technique (group A)was compared to a group of 30 kidneys harvested with a conventional hand suture (group B).

Results: In all kidney transplantations the vascular anastomosis were performed without technical or vascular complication. Duration of bench surgery differed significantly since group A had shorter time of kidneys harvesting (minutes, $34 \pm 8$ vs. $52 \pm$ $11, \mathrm{p}=.054$ ). We did not observe significant difference in terms of total vein elongation ( $\mathrm{mm}, 20 \pm 0.9$ vs. $19 \pm 1, \mathrm{p}=.496)$. No vein thrombosis was noted during the hospitalization.

Conclusion: In our experience,the elongation of the right renal vein using a vascular stapler technique proved to be easy and a safe alternative technique allowing to reduce the time of bench surgery and the organ cold ischemia. 


\section{8}

\section{Breast Cancer and Pregnancy}

\author{
A. Coglitore, F. Rovera, C. Piscopo, F. Frattini, S. Rausei, \\ L. Boni, G. Dionigi
}

Introduction: Pregnancy breast cancer (PBC) is one of the most common malignancy during pregnancy; up to 3\% of breast cancers are diagnosed in pregnant. As maternal age at the time of pregnancy continues to increase, the incidence $\mathrm{PBC}$ is expected to increase.

Materials and Methods: A review of the literature was performed in order to identify optimal treatment strategies.

Results: Most of the data about diagnosis and treatment PBC is small cohort studies without randomized controlled trials. Conservative surgery can be proposed at the end of second and third trimester and radiotherapy is delayed after childbirth. The safety of sentinel node biopsy has yet to be confirmed and the axillary dissection is the conventional treatment. The chemotherapeutic agents utilized are the same as those used in non-pregnant patients, but they should not be administered in the first trimester. Radiotherapy and endocrine therapy must be avoided during pregnancy.

Conclusion: The treatment of $\mathrm{PBC}$ needs active communication among the patient, obstetrician, medical, surgical and radiation oncologists. Diagnosis is often delayed due to breast changes; obstetricians should perform a thorough breast examination at the first prenatal visit with suspicion for cancer. Other therapies may need to be considered, although their usage now is not currently recommended owing to the paucity of safety data.

\section{9}

\section{Ectopic Breast Cancer: Case Report}

\section{S.A. Villari, F. Famà, C.C. Bramanti, A. Pollicino, P. Scarfò, M.A. Gioffrè Florio \\ Department of Emergency Care Unit, University of Messina, Italy}

Introduction: Ectopic breast tissue can be present anywhere along the "milk line," including the axillary region, as a consequence of incomplete resolution of embryologic mammary ridges. Mammary carcinoma arising in ectopic breast tissue is an uncommon occurrence. We present the case of a young woman who developed a carcinoma of ectopic axillary breast tissue.

Materials and Methods: A 31 year old woman presented two masses in the right and left axilla that had been present for 12 years. The patient reported that the axillary masses increased in volume and particularly the right mass become very erythematous during the last pregnancy (delivery occurred two months ago). Ultrasound was negative for neoplasm.

Results: The patient underwent a wide local excision of the two masses. Histological examination revealed an invasive ductal carcinoma in ectopic right axillary breast tissue. After a week, the patient underwent radical cancer surgery and right axillary lymph node dissection, with histological diagnosis of micrometastatic carcinoma in nine lymph nodes. Postoperatively, she was treated with radiotherapy and chemotherapy.

Conclusion: Cancer of the ectopic breast tissue is rare. Ectopic breast tissue is subject to the same hormonal influences and risk of disease as pectoral breast tissue and can undergo changes during pregnancy. Histologically, most adenocarcinomas arising in supernumerary breast tissue have been of the ductal type. The ectopic axillary breast mass prognosis is similar to the carcinoma of normal breast in the same tumour, node, metastasis stage, although it has a higher rate of lymph node involvement with a worse prognosis.

\section{0 \\ Risk for 24 Hours Mortality in Polytrauma: Our Experience}

\author{
S.A. Villari, F. Famà, LM. Murabito, C.C. Bramanti, \\ M.A. Gioffrè Florio \\ Department of Emergency Care Unit, University of \\ Messina, Italy
}

Introduction: Identification of mortality risk as a result of polytrauma is essential in a trauma system, because multiple injury results in a complex pathophysiological and immunological response, depending on the individual injury pattern, the time elapsed after injury and the systemic response. The aim was to identify incidence and risk factors associated with mortality for polytrauma in the emergency room.

Materials and Methods: We admitted 239 (26, 8\% red code and 73, 2\% yellow code) patients for polytrauma for the year 2009. Mean age 51 years. AIS, ISS, TRISS, GCS analysis were performed and the patients with an ISS of $\geq 16$ were included.

Results: The average time of hospitalization in the emergency room was 82 minutes. Eleven patients died. The 24 hours mortality was $2,1 \%$, the 30 days mortality was $1,7 \%$, the 60 and 90 days mortality was $0,8 \%$. No deaths occurred in emergency room. Five deaths were classified as preventable or potentially preventable. Mortality was strongly associated with head injury. In one case, death was due to crush syndrome.

Conclusion: The combination of critically ill and trauma scores may increase the accuracy of mortality prediction in polytrauma patients. The TRISS model can identify preventable deaths objectively. The role of diagnostic and therapeutic timely approach is very important to reducing the risk of mortality. 
111

\section{The Pneumothorax: Incidence and Mortality in the Emergency Room}

S. Surleti, F. Famà, S.A. Villari, G. Versace, F. Cucinotta, F. Armaleo, P. Placanica, M.A. Gioffrè Florio

Department of Emergency Care Unit, University of Messina, Italy

Introduction: Pneumothorax occurs when air from an injured lung or airway is trapped within the pleural space. It is classified as spontaneous (primary, secondary or catamenial) and traumatic (iatrogenic or secondary to blunt or penetrating chest injury). This study aimed to identify information on incidence, pathophysiology, management and mortality of Pneumothorax in Emergency Room.

Materials and Methods: Between January 2007 and December 2008, 73 patients with pneumothorax were admitted to our emergency room. The records of all patients were reviewed and data were retrospectively collected. Type of the pneumothorax, side, age, gender, incidence and mortality were analysed.

Results: In the period 2007-2008, we have recorded 73 cases (66 male and 7 female) of pneumothorax: 48 spontaneous (66\%), 24 traumatic (33\%) and 1 iatrogenic (1\%). The mean age was 47.3 years (range 12-99 years); the incidence of pneumothorax has been of the $0.11 \%$. Any death occurred during hospitalization in emergency room. Traumatic pneumothorax was associated with blunt chest trauma, pleural fluid, hemothorax, cranial trauma, clavicular fractures, upper and lower arm fractures, pelvic fractures, spinal/ vertebral trauma, sternum fractures and abdominal trauma.

Conclusions: Pneumothorax represents a common clinical problem. Multidisciplinary approach is necessary to decreasing morbility and mortality risks. In our experience the incidence of pneumothorax in emergency room is equal to the data presents in literature. While our data on the mortality are not comparable, because there are few studies about this subjects.

112

\section{Thoracic Trauma: Incidence and Mortality in the Emergency Department}

\section{S. Surleti, F. Famà, L.M. Murabito, S. AVillari, C. Malara, C. Estollere, A. Caruso, A. Beccarla, M.A. Gioffrè Florio \\ Department of Emergency Care Unit, University of Messina, Italy}

Introduction: Thoracic trauma is a major cause of hospitalisation in the world. Thoracic trauma comprises $10-15 \%$ of all traumas and represents $25 \%$ of all fatalities due to trauma. The incidence of trauma is various, and relatively higher numbers of chest injuries are observed for increasing traffic accidents.

Materials and Methods: Between January 2007 and December 2009, 469 patients with thoracic trauma were admitted to the emergency department in our hospital. The records of all patients were reviewed and data were retrospectively collected.
Type of the trauma, age, gender, incidence and mortality were analysed.

Results: Between January 2007 and December 2009 thoracic traumas were 469, (304 male and 165 female) and the mean age was 52.8 years (range 12-96 years). More than two rib fractures were diagnosed in 45 patients $(9.6 \%)$; sternum fractures were detected in 16 patients (3.4\%). The annual incidence for thoracic trauma was $6 \%$ and mortality recorded was zero in the emergency department.

Conclusions: Thoracic trauma is still a major cause of hospitalisation and it represents an important cause of morbidity and mortality in younger populations. Multidisciplinary approach and continuous training of all professionals involved in trauma care is necessary in the management of trauma cases for life saving and for decreases morbidity and mortality. In our experience the incidence of thoracic trauma is less to the data reported in the literature and the mortality recorded has been equal to zero.

\section{3}

Fistula-Associated Anal Mucinous Adenocarcinoma in Crohn's Disease: A Rare Case

\author{
A. Zefelippo ${ }^{1}$, B. Oreggia' ${ }^{1}$ D. Pettinari ${ }^{1}$, F. Botti ${ }^{1,2}$ \\ A. Carrara ${ }^{1-2}$, E. Contessini-Avesani1,2 \\ ${ }^{1}$ Fondazione IRCCS Cà Granda Ospedale Maggiore \\ Policlinico di Milano, UO Chirurgia Generale e d'Urgenza, \\ ${ }^{2}$ Università degli Studi di Milano, Dipartimento di Scienze \\ Chirurgiche
}

Introduction: Carcinoma on perianal fistula in Crohn's disease (CD) is rare and diagnosis is not usually suspected.

Methods: We report a case of fistula-associated anal mucinous adenocarcinoma in a patient with CD. We checked patient's medical and pathological records and reviewed literature.

Results: A 30 years old woman had been suffering from CD for 13 years. Disease was characterized by mild terminal ileitis and chronic perianal fistulae, treated by seton positioning. After pregnancy, she had perianal relapse. Pelvic MRI and endoscopy showed a posterior transsphincteric fistula and a large pelvic abscess, compressing the rectum. Abscess was drained and tough tissue was biopsied; histological result was mucinous adenocarcinoma of the anus. Abdominopelvic MRI and CT scan were performed for restaging $\mathrm{CD}$ and local disease: a large mass involving the pelvic floor and terminal ileitis were demonstrated. Abdominoperineal resection, en bloc resection of coccyx, inguinal linfectomy and ileocaecal resection were performed. Pathology report was pT4bN0 mucinous adenocarcinoma. Adjuvant chemoradiotherapy was planned.

Conclusion: In the reported case, diagnosis of fistula-associated anal adenocarcinoma was unsuspected due to age of patient and mild symptoms of CD. According to literature, patients with chronic perianal CD fistulae should undergo periodic cancer surveillance and be biopsied if suspected. Aggressive therapy is mandatory in cancer cases. 
114

\section{Relief of Double Incidentaloma: Clinical- Surgical Strategy Versus Instrumental Diagnostic Methods}

R. Gervasi, C.Siani, A.M. Lucisano, C.Folliero, D.P. Pafundi, F. Lazzaro, R. Sacco, A. Puzziello

Division of General Surgery, AOU Mater Domini, Magna Graecia University, Catanzaro, Italy

Introduction: An incidentaloma is a tumor found by coincidence without symptoms or suspicion.

Materials and Methods: We report a case of a male, 55 years-old patient subjected to abdominal CT scan for hematuria, with relief of right adrenal lesion. During our following laboratory and instrumental study, there resulted only a right pulmonary densification ("ground-glass areas"). At preoperative chest X-ray, we found instead an opacity suspected for pulmonary neoplasy; so we carried out new chest CT, that confirmed it. However we performed a right VLS adrenalectomy for an histological definition and a next therapeutic strategy; meanwhile, the patient underwent to further chest CT (unchanged) and WB-PET (pulmonary superabsorber lesion with SUV>8). Histological examination of the adrenal lesion resulted positive for adenoma and, after multidisciplinary consulting, suspecting a round atelectasis, we tried antibiotic and steroid therapy.

Results: After therapy, the chest CT showed only apical fibrous streaks.

Conclusions: Up to $7 \%$ of all patients over 60 may harbour a benign growth, often of the adrenal gland, which is detected when diagnostic imaging is used for the analysis of unrelated symptoms. The relief of an incidentaloma obliges a depth diagnostic instrumental study, that however can't replace clinical and surgical reasoning.

\section{5}

\section{Cholangiocarcinoma with a Background of HCV-Related Cirrhosis: Case Report and Review of the Literature}

\section{Politi, A. Lanaia, G. Di Mauro \\ Università degli Studi di Catania, Dipartimento di Chirurgia Generale, Sezione di Chirurgia Generale ed Oncologica, Direttore: Prof. F. Basile}

Introduction: Cholangiocarcinoma is a malignant neoplasm arising from the biliary epithelium. It is the second most common primary malignant tumor of the liver after hepatocellular carcinoma. Recently, chronic viral hepatitis or cirrhosis has been suggested to be involved in the pathogenesis of cholangiocarcinoma.

Materials and Methods: In December 2009, a 75 years-old woman with a history of HCV-related cirrhosis came to our attention. During ultrasound follow up an hepatic tumor was found at the seventh hepatic segment. The first suspected diagnosis was HCC, even if alpha fetoprotein was normal. At first step the patient was addressed to chemoembolization in order to confirm the diagnosis and start the treatment. The tumor kept Lipiodol for about a month; then the patient was referred to surgery for resection.

Results: The patient was treated by surgical resection of segments VI-VII; histological examination of surgical specimen showed a cholangiocarcinoma.

Conclusions: In cases of chronic hepatitis or cirrhosis caused by hepatitis $\mathrm{C}$ virus, the occurrence of cholangiocellular carcinoma must be considered in the differential diagnosis in addition to hepatocellular carcinoma. Imaging findings are often the key of diagnosis.

\section{6 \\ Use of Intraoperative Endoscopy to Localize Bleeding in the Small Intestine}

\author{
E. Ricciardi, A. Manzelli, S. Quaresima, P. Rossi, \\ O. Buonomo, G. Petrella
}

Policlinico Tor Vergata. Dipartimento di Chirurgia, Cattedra di Chirurgia Generale Università Tor Vergata, Direttore Prof. Giuseppe Petrella

Case Review: Bleeding within the small intestine is difficult to diagnose and localize because it typically occurs at a slow rate. These patients may undergo multiple transfusions and repeated endoscopy, contrast studies, bleeding scans, and angiography before the bleeding source is identified. We report a case of 64-year-old woman, where both endoscopic and angiographic techniques were used to localize protracted bleeding. During endoscopic treatment, the arteriovenous malformations continued bleeding. However, highly selective angiography and intraoperative endoscopy outlined the segments of small intestine for resection. This case reviews the evaluation, localization and treatment of small intestine bleeding.

Localizing the site of protracted bleeding in the small intestine beyond the duodenum bulb can be problematic. For some patients, the course of examinations and transfusions can take years. The small intestine is an uncommon site for gastrointestinal hemorrhage, and only $3 \%-5 \%$ of gastrointestinal bleeding occurs between the ligament of Treitz and the ileocecal valve. The length and location of the small intestine, along with other anatomical factors, make this area difficult to assess with endoscopy or radiology. In this case of protracted bleeding, highly selective angiography and intraoperative endoscopy were used to locate the source of the bleeding. 


\section{Laparoscopic Surgery}

\section{7}

\section{Quality of Life After Transanal Endoscopic Microsurgery and Laparoscopic Total Mesorectal Excision for Rectal Cancer}

\author{
M.E. Allaix, F. Rebecchi, C. Giaccone, M. Caldart, \\ G. Giraudo, F. Festa, M. Morino \\ Digestive, Colorectal and Minimal Invasive Surgery, \\ University of Torino - Corso Dogliotti, 14 - 10126 Torino, \\ Italy
}

2nd Classified - SPIGC Award Session, Laparoscopic Surgery

Introduction: From the oncological point of view, TME is the gold standard in the treatment of rectal tumors, with a quite high rate of postoperative morbidity, expecially in terms of urinary and sexual dysfunctions. To avoid the morbidity of TME, TEM is considered an option in the treatment of well-selected patients. Aim of this study was to compare quality of life (QoL) after TEM and laparoscopic TME.

Material and Methods: 42 patients underwent TEM for rectal neoplasms with a radical intent. The follow-up included EORTC QLQ-C30 and EORTC QLQ-CR38 questionnaries and Wexner score for fecal incontinence at 1, 3 and 12 months and anorectal manometry at 3 and 12 months. The results were compared with a group of 44 patients who underwent LTME.

Results: Compared with TEM, a significantly worse score was observed after LTME regarding role function $(p<0.001)$, sleep disturbance, appetite loss, constipation, weight loss $(\mathrm{p}<0.05)$. A trend towards better nausea/vomiting and nicturia score was seen after TEM. No significant difference was observed in Wexner score. The basal sphincter pression was significantly lower after TEM ( $p=0.001)$, without fecal incontinence.

Conclusion: QoL after TEM seems to be better than after LTME. The postoperative lower sphincter basal pression is not associated with incontinence. Further prospective large clinical trials are needed to confirm our suggestions.

\section{8}

\section{Pancreas Transection with Electrothermal Bipolar Vessel Sealer (Ligasure ${ }^{\circledR}$ ) in Laparoscopic Left Pancreatectomy}

\section{G.L. Baiocchi', A. Sartori², C. A. Sartori² \\ ${ }^{1}$ Surgical Clinic, Brescia University, ${ }^{2}$ Department of General Surgery, Castelfranco Veneto Hospital}

Introduction: Pancreatic fistula after distal pancreatectomy remains an unsolved problem (range 15-40\%).
Methods: Herein we present the case of a 68 year old woman with a $2,5 \mathrm{~cm}$ symptomatic insulinoma of the pancreas tail submitted to laparoscopic spleen preserving distal pancreatectomy. Exposure of the pancreatic neck was obtained by a medial to lateral approach; once the nodule was identified in the pancreatic surface, and confirmed by intraoperative ultrasonography, a tunnel underneath the pancreatic neck was created; then dissection of the pancreatic body was carried out. Pancreas neck transection was perfomed by $10 \mathrm{~mm}$ LigaSure $^{\oplus}$ : the pancreatic parenchyma was grasped in the jaws of the instrument and a calibrated force was applied to the tissue. Cutting was done only after multiple applications, irrespectively of the sound signaling that output is automatically discontinued. Haemostasis was obtained at the same time.

Results: The patient had a favorable outcome and was discharged in $6^{\text {th }}$ pod without complications.

Discussion: The reported technique presents the advantages of an easy employ and of a contemporary haemostasis, thus being particularly suitable by the laparoscopic way, and may offer from a theoretical point of view some advantages over conventional pancreatic stump closure techniques. A prospective clinical study has now been designed.

\section{9}

\section{Changing Trends in laparoscopy training Among Italian Residents}

\author{
L. Bencini, M. Calistri, G. Cavallina, C. Tommasi, \\ R. Sacchetti, C. Ureña, R. Moretti \\ Division of General and Oncologic Surgery, Careggi \\ Regional and University Hospital, Florence
}

Introduction: It is very difficult to gain sufficient proficiency in surgery for Italian residents. Moreover, no specific papers or surveys about this issue have been published so far, while, with the advent of laparoscopy, surgical training is of outmost importance to get adequate skills. A well organized schedule of rotations among referral Centers with Units of surgery with peculiar interest in the field of minimally invasive techniques is essential for an up-to-date General Surgery Residency Program.

Materials and Methods: A retrospective survey of laparoscopies performed by residents enrolled in the Unit of Oncologic Surgery of Careggi Main Regional and University Hospital, Florence, Italy was conducted. The aim of the survey was to identify any change in the attitudes of performing laparoscopy during the recent years. All residents certified from 2002 to 2009 were included, as those who were still in the program at the time of the survey.

Results: Among the residents who get their certification between 2002 and 2009 the average number of total procedures and laparoscopic procedures performed per year was 53 (43-78 \pm $13 \mathrm{SD})$ and $8(4-13 \pm 3 \mathrm{SD})$, while it was 61 (49-71 $\pm 12 \mathrm{SD})$ and 24 $(18-30 \pm 6 \mathrm{SD})(\mathrm{p}=0.2$ and $\mathrm{p}=0.006)$ respectively for those enrolled in our Center in the last years, who are still in the Program. The percentage of laparoscopies out of the total of procedures performed was $16 \%$ for the old residents vs. $39 \%$ for the younger group $(\mathrm{p}=0.006)$. 
Conclusions: Although not representative of the entire Italian Resident cohort, this survey shows how nowadays residents are performing much more laparoscopies as compared to residents certified some years ago. The total number of procedures, including both open and laparoscopic operations, remained unchanged.

\section{0}

\section{Single Incision Laparoscopic Wedge Resection of Gastric GIST}

F. Cantore, L. Giavarini, M. Di Giuseppe, E. Cassinotti, E. Colombo, S. Maria Tenconi, L. Boni

Università dell'Insubria - Milano, Italia

Introduction: Gastrointestinal stromal tumours (GIST) are the most frequent mesenchimal tumours of the gastrointestinal tract; they are often asymptomatic and the diagnosis is often during other exams. Surgical resection is the treatment of choice for GIST.

Materials and Methods: This video shows our technique for single incision laparoscopic wedge resection for a Gastric GIST of the greater curvature. The abdominal CT scan shows a small external lesion of the great gastric curve with a short vascularised pedicle. Patient was placed in supine decubitus in anti-trendelemburg position and the main surgeon is placed between patient's legs. The assistant is placed on the right side of the bed. We use open technique for pneumoperitoneum through the umbilicus and insert a $5 \mathrm{~mm}$ trocar in order to explore the abdominal cavity; the incision is enlarged vertically up to $2.5 \mathrm{~cm}$, the wound is protected using a "O" ring and a single incision port devices is inserted (ENDOCONE-tm by Karl Storz).

Results: The gastric fundus mobilization was achieved with harmonic scalpel in order to expose the pedicle. Small blood vessels to the tumour were coagulated by mead of bipolar electrocautery and wedge resection of the gastric curvature was accomplished using a $60 \mathrm{~mm}$ stapler with green cartridge. The surgical field is checked for bleeding and the lesion was then placed in bag and removed through the umbilicus after the ENDOCONE-tm was removed. No drainage of peritoneal cavity was necessary and the fascia was closed with re-absorbable suture.

Conclusion: SILS wedge-resection for Gastric GIST is feasible and safe.

\section{1 \\ Biliary Peritonitis Due to a Common Bile Duct Injury During Laparoscopic Cholecystectomy: A Lesson to Learned}

F. Cantore, M. Di Giuseppe, E.M. Colombo, L. Giavarini, S.M. Tenconi, E. Cassinotti, L. Boni

Università dell'Insubria - Milano, Italia

This video shows a case of choleperitoneum due to a common bile duct injury during a laparoscopic cholecistectomy. The patient, male, 56 years old, underwent laparoscopic cholecistectomy for sympromatic cholelitiasis; there were no peri-operative complications and the patient was discharged in second postoperative day. The sixth post-operative day the patient came to the Emergency Ward for abdominal pain; the CT scan demonstrated the presence of free fluid and gas in the peritoneal cavity; evidence of sub-diaphragmatic gas at the Xrays, WBC 11.000/mm3. The patient underwent an ERCP that showed a biliary leakage of the common bile duct near the metallic clips placed during the laparoscopic cholecystectomy; a biliary stent was then placed. The next day the patient underwent an explorative laparoscopy, with the evidence of a widespread biliary peritonitis caused by a common bile duct lesion that was identified and only partially solved by the biliary stent so, after peritoneal washing, $4 / 0$ suture of the lesion was performed and a drainage was positioned. Reviewing the video of the first procedure showed that the lesion of the bile duct was probably caused by electrical conduction due to accidental contact between the monopolar hook and the metallic clip placed on the cystic artery. The patient was discharged on day 5 after the second procedure and the second post-operative course was uneventful.

122

\section{Stapler-less Laparoscopic Splenectomy with Early Ligation of the Splenic Artery: Preliminary Experience}

D. Cavaliere, M. Framarini, D. Di Pietrantonio, F. Tauceri, F. D'Acapito, A. Vagliasindi, G. Solfrini, G.M. Verdecchia

U.O. Chirurgia e Terapie Oncologiche Avanzate Department of Surgery, "GB. Morgagni-C. Forlanini" Hospital, Forlì - Italy

We report our experience of laparoscopic splenectomies (LS) performed in a semi lateral approach with a stapler-less technique. Patients:Eleven consecutive LS were attempted for both benign $(n=8)$ or malignant $(n=3)$ disease. In order to minimize the haemorrhagic risks, we routinely performed an early ligation of the splenic artery with non-absorbable polymer clips (Hem-o-lok ${ }^{\circledast}$ ). In order to decrease the operative time, we used the ultrasonic scissor (Harmonic $\mathrm{ACE}^{\mathrm{m}}$ ). In an attempt to reduce the operative costs, we avoided the disposable surgical devices, such as trocars and staplers. Results:Conversion rate was nill. An accessory incision $(8-10 \mathrm{~cm})$ was required in 3 patients in order to remove the 
entire spleen for diagnosis. Splenic diameter ranged from 10 to 28 $\mathrm{cm}$. Massive splenomegaly (over $20 \mathrm{~cm}$ ) was present in 3 patients. Mean operative time was 208 min (range 112-305). Estimated blood-loss was $110 \mathrm{ml}$ (range 30-450). No significant intra-operative complications were observed. Two patients had an incomplete thrombosis of the portal venous system; anticoagulant therapy was initiated and complete recanalization was observed without any adverse event. Mean hospital stay was 5,7 days (range 4-11). Conclusions:The early artery ligation, the semi lateral approach and the harmonic scissor may determine a safe dissection, manipulation and removal of either normal size or bulky spleen.

\section{3 \\ Single Incision Laparoscopic Right Colectomy}

\author{
M. Di Giuseppe, L. Giavarini, E.M. Colombo, F. Cantore, \\ S.M. Tenconi, E. Cassinotti, L. Boni \\ Università dell'Insubria - Milano, Italia
}

Introduction: To describe the surgical technique for single incision laparoscopic right colectomy and present preliminary short term results.

Summary Background Data: Laparoscopic surgery has been fully validated as alternative, minimally invasive, treatment for different benign and malignant conditions. In the attempt to reduce even more the surgical trauma, natural orifices trasluminal endoscopic surgery (NOTES) and single incision laparoscopic surgery (SILS) have been proposed. While the lack of proper instrumentations makes NOTES not fully suitable for advanced procedures, SILS might play a significant role although, to date, only two case reports of single incision right colectomy are present in the literature.

Methods: Once obtained signed informed consent patients, patients with malignant tumor or large polyps of the right colon undergone to single incision colonic resection through a $3,5 \mathrm{~cm}$ incision using single port device and articulated instruments. Preliminary results were analyzed retrospectively.

Results: 14 patients were selected for SILS procedure, there were no intra-operative complications or need for conversion to standard laparoscopic procedure. One patient has post-operative urinary tract infection while no complications were reported in all the remaining cases.

Conclusion: In our experience SILS right colectomy is a safe and effective procedure.

\section{4 \\ Single Incision Laparoscopic Left Colectomy for Cancer}

M Di Giuseppe, E.M. Colombo, F. Cantore, L. Giavarini,

S.M. Tenconi, E. Cassinotti, L. Boni

Università dell'Insubria - Milano, Italia

Introduction: Laparoscopic surgery represent an effective alternative to traditional surgery. Recently technological innovations had as its target the reduction of surgical trauma. SILS could open a new prospect for mini-invasive surgery. We show our technique for single incision laparoscopic left colectomy for cancer.

Materials and Methods: The patient, with a cancer of the sigmoid colon, is placed in lithotomy position, the main surgeon and the assistant stand on the right side. A $3.5 \mathrm{~cm}$ umbilical incision is performed and an Endocone (Karl Story, Germany) single port device is introduced. We begin with the identification of left ureter, then isolation and division between clips of inferior mesenteric artery and vein is performed. The procedure carries out with the dissection of the Toldt's Fascia and mobilization of left colon. At this point we needed to add a additional trocar in the right iliac fossa to divide the high rectum using a mechanical stapler (Echelon 60, Ethicon endo-surgery). The procedure continues with the placement of a wound protector and extraction of the mobilized left colon through the incision. The left colon was then resected and the anvil of a $29 \mathrm{~mm}$ circular stapler was inserted. The Endocone was re-inserted and a colo-rectal end to end anasthomosis was performed. Final check for haemostasis and a drain was left in place.

Results: The length of the resected specimen was $42 \mathrm{~cm}$, and the final pathological report revealed a well differentiated adenocarcinoma T3, N0 with 32 lymphnodes removed.

Post operative course was uneventful and the patient was discharged on post-operative day 6 .

Conclusion: In our experience SILS left colectomy is a safe and effective procedure.

125

\section{Lesson Learned from Gasless Laparoscopic Adrenalectomy}

G. Giraudo, F. Festa, F. Famiglietti, V. d'Adamo, M. Morino

Digestive, Colorectal and Minimal Invasive Surgery, University of Torino

Introduction: Pneumoperitoneum represents a contraindication in several clinical conditions. Gasless technique could be an attractive solution to perform minimally invasive procedures in these cases. We report 11 cases of gasless laparoscopic adrenalectomies (LA) successfully performed in patients with contraindications to pneumoperitoneum.

Material and Methods: All gasless LA were performed transperitoneally in lateral flank position, using the LaparoTenser (L\&T Lucini, Milan, Italy) as abdominal wall retractor with two 
curved needles (Aghi Pluriplan) placed in the subcutaneous tissue of the abdominal wall, that allows both gasless and low-pressure pneumoperitoneum avoiding negative effects of high intrabdominal pressure.

Contraindications to pneumoperitoneum were: 4 vascular intracranic malformations, 1 acute glaucoma, 1 severe pulmonary emphysema, 2 hypertensive retinopathy, 1 recent neurosurgical intervention and history of vascular cerebral accident in 2 cases.

Results: From March 1995 to December 2009, 355 LA were performed. Among these, 11 (3.1\%) were gasless LA: left, right and bilateral resections were performed in 5, 4 and 1 cases respectively. Histopatological findings revealed 5 cortisol producing adenomas, 3 pheochromocytomas, 2 aldosteronomas and 1 nonfunctioning adenoma. Mean lesion size was $2.87 \mathrm{~cm}$. Mean operative time was 70.9 minutes. There were no conversions to open surgery. Mean postoperative hospital stay was 3.6 days. There were neither intraoperative nor postoperative complications.

Conclusion: Gasless technique is safe and feasible allowing the well known advantages of laparoscopy in patients with contraindications to pneumoperitoneum.

\section{6 \\ Laparoscopic Adrenalectomy for Pheochromocytoma}

\author{
G. Giraudo, F. Festa, F. Famiglietti, V. d'Adamo, M. Morino \\ Digestive, Colorectal and Minimal Invasive Surgery, \\ University of Torino
}

\section{1st Classified - SPIGC Award Session, Laparoscopic Surgery}

Introduction: Since 1992, laparoscopic adrenalectomy (LA) appeared the gold standard to treat benign adrenal disorders.

We present a single center experience of LA for pheochromocytoma.

Material and Methods: This study represents a retrospective analysis of a prospectively collected database of all LA for suspected pheochromocytomas from a single center. From March 1995 to December 2009343 patients underwent LA. Suspected pheochromocytoma was the indication for surgery in 50 patients. All LA were performed transperitoneally in lateral flank position.

Results: Most diagnosis were for symptomatic disease (27/50). Left, right and bilateral LA were performed in 24, 22 and 4 cases respectively. There were no laparotomic conversions. Mean operative time was 95.6 minutes. Mean lesion size was $3.98 \mathrm{~cm}$. Histopatological findings revealed a pheochromocytoma in 46 cases (85.2\%). There were four intraoperative complications (8\%): three cases of hypertensive crisis and one case of acute pulmonary oedema. The postoperative morbidity rate was $14 \%$ (7/50): two cases of hypertensive crisis, three cases of respiratory insufficiency and two cases of bacterial pneumonia all successfully medically treated. Perioperative mortality was null.

Postoperative improving and/or resolution rate of hypertension was $94 \%$ and in 3 cases (6\%) blood pressure values remained unchanged. The postoperative control of Chromogranine A, uri- nary metanephrines and plasmatic catecholamines ranged in the normal values in $94 \%$ of patients (47/50).

Conclusion: LA is a feasible and safe technique for the treatment of pheochromocytoma. Nevertheless, a multidisciplinary approach involving endocrinologists, anaesthesists and surgeons in Centers highly experienced into endocrine and advanced laparoscopic surgery is mandatory.

\section{7}

\section{Laparoscopic vs. Open Surgery: Treatment of Median Incisional Hernia}

\author{
F. Coratti, R. Malatesti, G. Colasanto, L. Gazzabin, \\ M. Bucalossi, M. Lo Gatto, F. Varrone, A. Coratti, F. Tani, \\ A. De Martino
}

Department of General Surgery, University of Siena, Hospital "Santa Maria alle Scotte", "Department of General Surgery, Grosseto, Hospital "Misericordia"

Introduction: In the last ten years we assisted to spreading of laparoscopic approach to correction of median incision hernia, although for increased interesting toward mini-invasive techniques than for matching results between lap and open approaches.

Materials and Methods: The aim of our study is the critical analysis of the results of lap and open surgery in the approach to ventral hernia, through the revision of the principal checked prospective trials.

Results: From revision of literature 7 perspective studies have emerged to fit to an analysis, for a total of 1165 patients. Among the perioperative outcomes has been observed, with statistically significance, briefer surgical time and a reduction of postoperative hospitalization for the lap. Statistically significant differences have not emerged neither on the other surgical end-points, neither on recurrences of hernias at 1 and 5 years.

Conclusion: Laparoscopy can be considered a valid technical alternative to traditional open surgery in the treatment of ventral incisional hernia.

Advantages of mini-invasive approach are less surgical time and reduction of total hospital stay.

\section{8 \\ Laparoscopic Treatment of Perforated Diverticular Peritonitis}

\section{P. Tarchi, A. dell' Antonio, G. Giacomel, N. de Manzini \\ Department of General Surgery, University of Trieste, Trieste, Italy}

Introduction: The classical treatment of peritonitis due to colonic diverticular perforation is based on major surgical interventions, such as Hartmann's, resulting in high morbidity and mortality. The presence of peritonitis has previously been considered a contraindication for the laparoscopic approach. 
The aim of this study is to define when the laparoscopic treatment is indicated.

Materials and Methods: Ten patients with peritonitis due to complicated diverticular disease underwent first an urgent laparoscopic irrigation and drainage and subsequently a laparoscopic anterior resection.

Patients were classified and selected on the basis of the peritonitis' gravity and general conditions: patients with poor general conditions, faecal peritonitis, shock, bowel obstruction and multioperated of major abdominal surgery were excluded.

Antibiotics and parenteral fluids administration were started, continued for one week and followed by oral antibiotics intake.

The laparoscopic exploration confirmed the diagnosis and allowed a careful peritoneal toilette and drainage.

No complications or early re-interventions have occurred. In one case a faecal low-flow fistula has persisted on the drainage location. All patients were discharged between the seventh and the tenth post-operative day. The conversion rate and mortality were null.

No new attacks of acute diverticulitis or secondary abscesses have been observed. All patients have been operated of laparoscopic anterior resection with success after one month.

Conclusion: The laparoscopic treatment of local and generalized peritonitis due to complicated diverticular disease is safe in selected patients. The subsequent elective laparoscopic resection results in a reduction of the morbidity and mortality and better quality of life, avoiding a stoma.

\section{9}

\section{Non-Traumatic Splenic Rupture Related to a Cavernous Hemangioma}

\section{U. Grossi, G. D'Amato, P.M.C. Tomaiuolo, A. Mazzari, \\ R. Menghi, A. Crucitti, R. Bellantone \\ Department of Surgery, Catholic University of Rome}

Introduction: Non-traumatic rupture of the spleen is uncommon, representing about $10 \%$ of all splenic ruptures. As a leading cause, hemangioma of the spleen (HS) has been rarely reported, accounting for up $0.75-1.6 \%$ and only described in case reports or few series. It generally occurs in adults, with a male to female ratio of 3:1.

Materials and Methods: A 41-years-old caucasian man was referred to our Department for acute pain in left hypocondrium irradiated to the left ribcage after a whooping cough. A well-defined $4 \times 4.8 \mathrm{~cm}$ hypodense high polar round lesion of the spleen was documented at CT scan. A selective splenic arteriogram was performed, showing a partially avascular, non-bleeding high polar lesion. The patient underwent laparoscopic splenectomy.

Results: Histopathological analysis of the spleen showed a $4 \mathrm{~cm}$ high polar hemorrhagic nodule surrounded by splenic parenchyma with blood spillage, referring to a splenic cavernous hemangioma. The post-operative course was uneventful and the patient was discharged on the $6^{\text {th }}$ post-operative day.

Conclusion: Non-traumatic splenic rupture should be considered in patients presenting with abdominal pain and hypoten- sion even without a history of trauma, as a prompt diagnosis and early intervention can be life saving.

\section{0}

\section{Our Experience About Laparascopic Appendicectomy: Is the Cochrane Review Right?}

\author{
A. Lucchi, P. Berti, M. Salvi, F. Vandi, F. Maffi, J. Brandolini, \\ C. Magnani, G. Garulli \\ Uo Chirurgia Generale E Toracica - Presidio Ospedaliero Di \\ Riccione - Ausl Rimini
}

Introduction: Laparoscopic appendicectomy (AL) isn't established yet like a gold-standard for the treatment of the acute appendicectomy (AA).

In this retrospective study we have analysed more than 250 cases of appendicectomy.

Materials and Methods: In the three years 2007-2009 we operated $340 \mathrm{AL}$ in succession, in the cases of a suspected AA. We used a standard surgical technique that provides 3 trocars and the use of mechanical suturation or loops.

Results: The medium operating time was of 46 minutes (15$180-+21)$. The medium stay in hospital of 2,7 days (1-22.+-2,59). It was necessary to change in ad open for the $9,1 \%$ of the cases. The mortality was represented for 1 case $(0,29 \%)$. It was the case of a gangrenous appendicitis in a obese and old patient with comorbility where it was necessary to change in ad open.

The total complications were $24(7 \%)$ and $13(3,7 \%)$ of these ones were of specific nature. In 7 cases $(2 \%)$ it was necessary to have a new operation. In 10 cases $(2,9 \%)$ it was necessary to hospitalize again the patients in a month after the operation.

Conclusions: Many studies have showed that the AL presents some benefits in a shorter stay in hospital, the post -operating pain, infections of the injury. After the analysis of our experience data, we can conclure that the AL can be considered like the elective approach in the case of a suspicious AA, and so not only for female and obese patients.

\section{1}

\section{Gallbladder Lymphoma: Case Report. Laparoscopic Treatment}

\section{Marenco, M. Castagnola, G. De Silva, E. Ciferri, G.S. Bondanza \\ Surg. Dept. Genoa North Hospital, Chief Prof. G.S. Bondanza}

Introduction: Lymphoma of the gallbladder is very rare. It can be divided into MALT (more frequent), follicular or as secondary involvement of gallbladder by NHL.

Material and Methods: A 79 years old male came to our observation for a precordial pain, irradiated to epigastric region 
and moderate diarrhea over the past three months. It was excluded ischemic heart disease. The upper endoscopy has revealed esophagitis with hyatal hernia with gastric erosions. Colonscopy has showed diverticulosis of the sigmoid-descending. On the basis of an abdominal ultrasound, which reported a thick gallbladder wall with many stones inside, suspected common bile duct stones and some adhesions between the gallbladder and duodenum, we submitted the patient to a laparoscopic cholecystectomy, with adhesion lysis and intraoperative cholangiography. In 2nd p.o. day one residual biliary tract stone was removed by ERCP and PSP and the patient was discharged on the $5^{\text {th }}$ p.o day.

Results: Histopatological examination of the gallbladder has showed a B-cell lymphoma. We completed the staging with a total body computed tomography (CT) that has demonstrated multiple enlarged abdominal and thoracic lymph-nodes.

We performed also a palpable axillary node and a bone marrow biopsy. Finally the patient was treated with systemic chemotherapy (R-CHOP).

Conclusion: Lymphoma could be remained silent when systemic disease is already an advanced stage.

This case suggests that it might be suspected when the gallbladder wall is thick, in the absence of acute cholecystitis. The overall assessment of the patient (CT, Upper endoscopy, nodes and bone marrow biopsy) is necessary to set the therapeutic strategy.

\section{2}

\section{Our Original Technique of Laparoscopic Right Hemicolectomy with Caudo-cranial Dissection and the Resuts of First 124 Consecutive Cases}

F.S. Mari, L. Masoni, R. Maglio, A. Milillo, A. Pancaldi, F. Pindozzi, A. Dall'Oglio, G. Berardi, A. Brescia

Azienda Ospedaliera Sant'Andrea, Università La Sapienza di Roma, II Facoltà di Medicina E Chirurgia

Usually, a medial to lateral technique is used to perform Laparoscopic right hemicolectomy (LRH). Our original technique is based on a caudo-cranial dissection. We first identify the ileocolic vessels and dissect them, then we open the mesentery to the last ileal loop and dissect the latter with an EndoGIA. Pulling the last ileal loop upward, we open Houston's ligament and begin the dissection of the colon following Toldt's fascia up to the hepatic flexure. Mobilized the right colon, is then opened the hepatogastro-duodeno-colonic ligament. At this point, pulling the bowel cranially, we identify and divide the right colic vessels and Henle's venous branch. In fat patients, the dissection of the ileocolic vessels is preceded by the dissection of right colon from the retroperitoneum up to the $3 \mathrm{rd}$ portion of duodenum. The anastomosis is performed using EndoGIA. From January 2003 to December 2009 we used this technique in 124 pts: 69 men and 55 women. Mean age: 68 yrs (37-88). Mean BMI: 26.6 (22.3- 37.4). The average time of the operation was $125 \mathrm{~min}$, with no conversions. Mean postoperative hospitalization was 5.3 days. In four cases we had an anastomotic leak (3.2\%). LHR is a simple and reproducible technique and it allows to reduce operative time in obeses.
133

\section{Transvaginal Cholecistectomy, a New Way to Treat the Cholelithiasis in Women}

\author{
F.S. Mari, L. Masoni, R. Maglio, A. Milillo, A. Pancaldi, \\ F. Pindozzi, A. Dall'Oglio, G. Berardi, A. Brescia
}

UO Day Surgery, Azienda Ospedaliera Sant'Andrea, Università La Sapienza di Roma - II Facoltà di Medicina e Chirurgia

Today the major innovation in surgery is the introduction of the Natural Orifice Transluminal Endoscopic Surgery but actual instrumentation not allows to perform surgical procedures simply and safely. To evaluate the possibility of this kind of surgery for cholecistectomy we have developed an "hybrid" procedure using one umbilical trocar and transvaginal access. From March 2008 to December 2009 we performed 21 transvaginal cholecistectomy (TVC) with the last 16 cases treated in Day Surgery setting. The TVC inclusion criteria were an age below 65 years, a BMI under 30 , an anesthesiological low risk (ASA I or II), previous pregnancies carried to term, a good patients motivation and none acute gallbladder disease nor previous abdominal surgical procedure. This operation is performed inserting only a $5 \mathrm{~mm}$ trocar in the umbilicus and performing a $1.5 \mathrm{~cm}$ incision on posterior vaginal fornix to insert an HD Colonoscope and $45 \mathrm{~cm}$ Grasper. The main operative time is about 60 minutes, no conversion to open surgery is needed. Patients are discharged 8-10 hours after surgery in good general conditions, without abdominal and vaginal pain. Controlled after one week, the patients confirmed the absence of significant post-operative pain and especially stressed the absence of pain and cosmetic result positively. No one complication to vaginal incision was observed.

\section{4 \\ Laparoscopic Treatment of a Carcinoma of the Cecum Incarcerated in a Right Groin Hernia Report of a Case}

\author{
M. Igor, P. Graziano, A. Giovanni, B. Bruno, F. Francesco, \\ D. Annibale
}

Minimally Invasive and Robotic Surgery Unit - San

Giovanni - Addolorata Hospital - Rome

Introduction: A carcinoma in a groin hernia is uncommon. We report a case of an intrasaccular carcinoma of the cecum in a right inguinoscrotal hernia with a simultaneous left inguinal hernia treated by laparoscopic approach.

Materials and Methods: A 70-years-old-man presented with a painful, not-completely-reducible bilateral hernia. Blood exams showed anemia. A CT-scan of the abdomen confirmed the presence of the ascending colon and some ileal loops into the hernia sac, showing a round-wall-thickening of the herniated portion of the right colon. A standard laparoscopic right colectomy with radical oncological purpose was performed. 
Conclusion: Incarcerated inguinal hernia is a relatively common surgical problem. In case of anemia or other signs suggestive for malignancy, a specific preoperative work-up should be assessed. This case demonstrates that it's possible to perform an oncologically-correct laparoscopic resection when the presence of malignancy is confirmed and an open traditional hernioplasty to avoid any possible contamination of the mesh.

\section{5 \\ Laparoscopic Peritoneal Lavage for Perforated Colonic Diverticulitis}

\author{
S. Rampado, R. Cresti, A. Cuviello, A. Donfrancesco, \\ A. Basso, L. Griggio \\ Complex operating unit of General Surgery Hospital, \\ Arzignano, U.L.S.S. 5, "West Vicentino"
}

\section{3rd Classified - SPIGC Award Session, Laparoscopic Surgery}

Introduction: Hartmann's procedure is the standard approach to acute diverticulitis complicated by perforation. Laparoscopic peritoneal lavage, as first step, can control the intraabdominal sepsis and bring to the defininitive procedure, in election, avoiding a laparotomy and stoma. We have considered this approach in a selected group of patients.

Materials and Methods: Patients with perforated acute diverticulitis, undergoing laparoscopy surgery, as first step, formed our study population.

Results: Seventeen patients were included (10M: 7F), with a median age of 56 years (IRQ $=48-69)$. In all patients there was a radiological diagnosis of perforation. Three patients presented with Hinchey class IIb; 13 class III; one was class IV. In 8 patients (47\%), the perforated diverticula was sutured. The median hospital stay was $7.5($ IRQ $=6.8-9.3)$. Two $(12 \%)$ patients experienced early recurrence of symptoms. Twelve patients (71\%) underwent sigmoid resection: 11 (92\%) laparoscopically, a median of 90 days $($ IRQ $=45-120)$, after the initial laparoscopy, $1(8 \%)$ via laparotomy (the patient had a recurrence of diverticulitis on the 3 rd day after lavage). Two (12\%) patients were not operated for morbid conditions that contraindicated surgery. Three (18\%) patients are on a waiting list for surgery. The mortality was nil; the morbidity rates was 6\%: hyperpyrexia with persistent abdominal pain in one patient.

Conclusion: Laparoscopic approach, as first step, is safe and feasible in perforated diverticulitis in patients Hinchey class IIb-III. Laparoscopic resection of the pathological colon, in nonemergency conditions, is easier when the inflammatory process is turned off.

\section{6}

A New Standardized Technique for Laparoscopic Cholecystectomy: The Critical View of Safety. A Single-Centre Cohort Comparison with Infundibolar Approach

\author{
N. Vettoretto, C. Saronni, M. Giovanetti \\ Chirurgia Generale, A.O. M.Mellini - Chiari (BS) - Italy
}

Introduction: Laparoscopic cholecystectomy (LC) carries a $0.3-0.5 \%$ morbidity due to major biliary injuries. The majority of surgeons routinely performs LC with the so called "infundibolar" approach (IA), diffused and standardized in the nineties. Strasberg introduced the "critical view of safety" approach (CVSA), only recently inserted in controlled studies. It is characterized by a blunt dissection on the sub-serosal plan to obtain medialization of the artery and the opening of a new safety triangle, delimited superiorly by the medial gallbladder wall, inferiorly by the cystic duct, and medially by the cystic artery. This upper part of Calot's space does not comprehend arterial or biliary anomalies and is therefore ideal for a safe dissection.

Materials and Methods: The precept of CVSA-LC has been applied and compared with the IA-LC in a retrospective cohort study. We divided 174 patients into two groups who, respectively, underwent CVSA-LC performed by a young surgeon, and IA-LC performed by an experienced surgeon. Outcome values and operative times were achieved either for cholelithiasis, acute or chronic cholecystitis, and examined with univariate analysis (Student's $t$ test).

Results: No difference was evidenced in terms of morbidity and outcome; significant differences were found in operative time, favouring CVSA-LC in every stage of gallbladder disease.

Conclusion: We purpose this technique as the gold standard for LC teaching as it permits a significant lowering of biliary and hemorrhagic complications, a shorter operative time, and a standardized method both for complicated and uncomplicated gallbladder lithiasis.

\section{7}

\section{Left Hypochondrium Insertion of Verres Needle for Laparoscopic Surgery}

\author{
A. Marrosu, F. Serventi, P. Paliogiannis, M.A. Sassu, \\ D. Giangrande, F. Attene, M. Trignano, C. De Nisco*, \\ F. Badessi* \\ Surgical Pathology Institute, University of Sassari, Italy, \\ *Department of General Surgery, San Francesco Hospital, \\ Nuoro, Italy
}

Introduction: The Verres needle, invented in 1938, remains to date the principal surgical instrument for the induction of pneumoperitoneum (p.p.) in laparoscopic surgery. Although the diffusion and optimization of the insertion technique, positioning of the needle is not free of complications. This multicentric study compares the complications of Verres needle positioning 
in the traditional paraombelical site and alternatively in the left hypochondrium.

Methods: From January 2005 to December 2009 we performed 370 laparoscopic procedures. In all cases the p.p. was induced with the Verres needle positioned in left hypochondrium. Retrospectively we examined all reports for intra-operative complications due to the induction of the p.p.

Results: We observed three complications: one spleen puncture, one gastric puncture and one pneumothorax. The first two did not need any treatment, while the third was resolved with a temporary thoracic drain. Non of these complications required a conversion to a laparotomic approach.

Conclusions: The most frequent complications published for the paraombelical insertion are: ileal punctures and minor or major vessel injury. Positioning of Verres needle in the left hypochondrium is an effective and safe procedure for the induction of p.p. and presents less and irrelevant complications compared to its' insertion in the paraombelical site.

\section{8 \\ Laparoscopic Management of Hinchey 2 Acute Diverticulitis}

F. Trotta, D. Galbiati, F.P. Tinozzi

General and Thoracic Surgery Unit, Ospedale Maggiore, Lodi

Introduction: A case of laparoscopic 2-step approach (I abscess drainage, II sigmoidectomy) to diverticulitis is presented.

Materials and Methods: A 50-year-old obese man suffering from left lower quadrant abdominal pain, diarrhoea and fever was admitted to the surgical ward.

An abdominal CT showed perisigmoid inflammation (Hinchey 1a sigmoiditis).

Medical therapy was administered. Seven days after a control CT detected a left ilio-psoas muscle abscess (Hinchey 2).

At laparoscopy the sigmoid was adherent to small intestine and to the left parietocolic gutter. The sigmoid was mobilized by blunt dissection in order to expose the anterior abscess wall. Then the abscess cavity was drained and washed with saline. Two drainage tubes were placed (abscess cavity, recto-vesical pouch).

Results: Post-operative course: uneventful. Discharge: $14^{\text {th }}$ p.o. day. An abdominal CT ( $10^{\text {th }}$ p.o.d.) showed optimal abscess drainage. This finding was confirmed by a $4^{\text {th }}$ p.o. month CT. A $3^{\text {rd }}$ p.o. month colonscopy showed chronic sigmoiditis. A laparoscopic sigmoidectomy was planned, but the patient was admitted to an other Hospital due to a psychotic crisis and during that hospitalization he underwent open sigmoidectomy (uneventful course).

Conclusion: Laparoscopic 2-step management of diverticulitis can provide good functional results, rapid recovery time and can avoid stoma confection.

\section{Oncologic Surgery}

\section{9 \\ Effect of Short Course Preoperative Radiotherapy and Long Interval Before Surgery on Local Control in Locally Advanced Rectal Cancer}

\author{
E. Cardone, B. Pecori, A. Avallone, F. Tatangelo, D. Scala, \\ C. Sassaroli , F. Cremona, P.Delrio \\ Department of Coloproctology, National Institute of \\ Tumors "G.Pascale", Naples
}

Introduction: Preoperative short course radiotherapy (SCR$5 \times 5 G y)$ followed by surgery reduces local recurrence in locally advanced rectal cancer. Downstaging could be obtained increasing the interval between radiation and surgery. We investigated the effect of long interval before surgery after SCR in patients with resectable rectal cancer.

Material and Methods: Thirty-five patients with high risk rectal cancer received preoperative SCR. Surgery was planned 4/6 weeks after completion of radiotherapy. Response to radiation therapy was measured by tumour regression grade on surgical specimen.

Results: All patients completed the scheduled treatment. Pre treatment staging was as follow: $13 \mathrm{~T} 3 \mathrm{Nx}, 16 \mathrm{~T} 3 \mathrm{~N} 0,4 \mathrm{~T} 3 \mathrm{~N} 1,2$ T2N0. Mean distance from anal verge was $6 \mathrm{~cm}$. Mean interval between SCR and surgery was 44 days. An AR was performed in 29 patients, an APR in 6. Postoperative complications occurred in 16 patients. Two patients (5,7\%) died postoperatively. Downsizing occurred in all cases, downstaging in 28 cases (80\%). TRG was as follow: 3 TRG1,7 TRG2, 11 TRG3, 12 TRG4, 2 TRG5. At a median follow-up of 31 months 3 patients died of disease, 6 are alive with disease, 24 are alive and free of recurrence.

Conclusion: Long interval before surgery after SCR downsizes rectal cancer. Complete response is rare. TRG1-2 scores seem to be increasing with an interval to surgery larger than six weeks.

\section{0 \\ ESCO Trial: Colonic Stent Versus Emergency Surgery in Malignant Colonic Occlusion, an Interim Report

\author{
M.E. Allaix ${ }^{1}$, A. Arezzo ${ }^{1}$, C. Balagué2, E.M. Targarona², \\ M. Morino ${ }^{1}$ \\ ${ }^{1}$ Digestive, Colorectal and Minimal Invasive Surgery, \\ University of Torino - Corso Dogliotti, 14 - 10126 Torino, \\ Italy, ${ }^{2}$ Fundacio Institut de Recerca de l'Hospital de la \\ Santa Creu i Sant Pau - Barcelona, Spain
}

Introduction: Emergency surgery for acute colonic obstruction is burdened by a significantly higher rate of complications. 
The clinical employment of self-expandable metal stents today represents a valid alternative.

Materials and Methods: Patients with acute left-sided colonic cancer obstruction are randomly assigned to one of the two treatment groups: A) emergency positioning of a stent followed by elective surgery (laparoscopic or open); B) emergency surgery. Primary endpoint is the incidence of postoperative complications at 2 months. Secondary endpoints are postoperative mortality, success percentage of stent positioning and percentage of complications related to stent positioning, hospital stay, QoL, quantity of life, cost differences, oncological results.

Results: Since March 2008, 48 patients have been included: 22 in group A and 26 in group B. The morbidity rate is nil. The stents were positioned successfully in all but $3(13.6 \%)$ cases: in 2 the positioning was technically impossible, in 1 the patient had no restoration of bowel function. In all cases, emergency surgery was successfully performed.

Conclusion: Expected results consist in a reduction of postoperative complications and hospital stay in patients submitted to stent positioning without worsening of the evolution of the neoplastic illness. The available interim data don't permit to draw any conclusion yet.

\section{1}

\section{Prognostic Value of Preoperative D-Dimer Level in Colorectal Cancer Patients: Comparison with CEA}

\author{
Y. Macchitella, D. Marrelli, G. Cerullo, C. Pedrazzani, \\ G. Flauti, S. Caruso, A. Neri, F. Mariani, A. De Stefano, \\ F. Roviello, E. Pinto
}

Department of Human Pathology and Oncology, Section of Surgical Oncology, University of Siena, Italy

Purpose: To evaluate the clinical impact of preoperative D-dimer (DD) level as a tumor marker in patients with colorectal cancer (CRC). The prognostic value of preoperative DD assessment was evaluated in relation to CEA levels.

Methods: DD and CEA levels were assessed preoperatively in 199 patients who underwent resection for CRC and the results were analyzed statistically.

Results: Preoperative mean $( \pm \mathrm{SD})$ values of DD and CEA were $347.5( \pm 940.1) \mathrm{ng} / \mathrm{mL}$ and $106.4( \pm 1099.2) \mathrm{ng} / \mathrm{L}$, respectively. DD level significantly correlated with nature of surgery (emergency vs. elective; $P=0.002$ ), presence of residual tumor (R1-2 vs. R0; $P=0.037)$ and tumor diameter $(P<0.001)$. Conversely, DD levels were not associated with tumor grading, $\mathrm{pT}, \mathrm{pN}$ and $\mathrm{M}$ stages as well as Dukes classification. The 5-year survival rates were $80 \%$ and $64 \%$ for patients with negative and positive DD values $(P=0.156)$.

CEA levels significantly related to all major prognostic factors ( $\mathrm{R}$ actegory, pT, pN and $\mathrm{M}$ stages as well as Dukes classification). A significant worse prognosis was observed for patients with CEA levels above the cut-off value ( $95 \%$ vs. $49 \% ; P<0.001)$.

Conclusions: The possible clinical usefulness of preoperative assessment of DD plasma levels proposed by previous experi- ences has not been confirmed by our data. CEA confirmed to be the most reliable and valid indicator of prognosis.

\section{2}

\section{Transanal Endoscopic Microsurgery for Rectal Neoplasms. Experience of $\mathbf{3 0 0}$ Consecutive Cases}

M.E. Allaix, A. Arezzo, M. Caldart, F. Festa, M. Morino

Digestive, Colorectal and Minimal Invasive Surgery, University of Torino - Corso Dogliotti, 14 - 10126 Torino, Italy

Introduction: Abdominal resection for rectal neoplasms is associated with significant morbidity. Local excision with retractors can be proposed only for distal rectal lesions. With this retrospective review of our prospective series of transanal endoscopic microsurgery (TEM) procedures, we wanted to verify the advantages of local treatment in terms of disease recurrence and complication rates.

Materials and Methods: Indications for TEM were adenoma, early carcinoma, rectal ulcer, carcinoid tumor, GIST and leiomyosarcoma located in the extraperitoneal rectum. We analysed operating time, morbidity and mortality rates, length of hospital stay, staging discrepancy, recurrence rate and oncological outcome.

Results: From January 1993 to January 2007, 300 patients underwent TEM at our Institution. The mean operating time was 66 minutes. The peritoneum was inadvertently opened in 13 cases. The overall morbidity rate was $7.7 \%$. The mean hospital stay was 5 days. Histology demonstrated cancer in 90 cases. At a mean follow-up of 60 months, the recurrence rate was $0 \%$ in pT1, 24\% in pT2, and $50 \%$ in pT3. The overall estimated five-year survival rate was $87 \%$, and the disease-free survival rate was $82 \%$.

Conclusion: TEM is safe and effective in the treatment of adenoma and pT1; it carries a lower morbidity and a recurrence rate comparable to that of conventional surgery.

\section{3}

\section{TEO (Transanal Endoscopic Operation) for Rectal Lesions Using Two-dimensional Screen Visualization and Standard Laparoscopic Instruments}

\author{
M.E. Allaix, A. Arezzo, F. Festa, M. Morino
}

Digestive, Colorectal and Minimal Invasive Surgery, University of Torino - Corso Dogliotti, 14 - 10126 Torino, Italy

Introduction: TEM can be considered the gold standard for benign and early rectal neoplasms. Nevertheless the technical difficulties linked to the necessary training and the costs of the instrumentarium have limited its diffusion. Recently a different 
device named TEO has been proposed by Karl Storz SRL. The video demonstrates the way of use of the device focusing on pros and cons of the system.

Materials and Methods: TEO instrumentarium includes a $15 \mathrm{~cm}$ rectal tube with 3 working channels $(12,5$ and $5 \mathrm{~mm}$ ) for conventional laparoscopic instruments, plus a $5 \mathrm{~mm}$ channel dedicated to a $30^{\circ}$ optic. The rectoscope is connected to the operating table via a holding arm consisting of 3 joints and a single screw. The system is used in combination with a standard laparoscopic column: image is projected on a screen, insufflation is obtained by a conventional $\mathrm{CO}_{2}$ thermo-insufflator.

Results: The video illustrates the technique of dissection and suturing of the rectal defect. The particular shape of the tip of the rectoscope allows in most of cases to keep the patient in a supine position, no matter the location of the neoplasm, minimizing patient preparation, which sometimes is consistently time consuming.

Conclusion: TEO can be considered an excellent device to treat benign and early rectal lesions. Further clinical series and randomized studies will need to verify this initial experience.

\section{4}

\section{Neurotensin Conjugated Branched Peptides for Selective Targeting of Pancreatic} Adenocarcinoma

\author{
L. Bencini ${ }^{1}$, C. Falciani ${ }^{2}$, B. Lelli ${ }^{2}$, J. Brunetti ${ }^{2}$, A. Pini ${ }^{2}$, \\ C. Pagliuca ${ }^{3}$, N. Ravenni ${ }^{2}$, S. Menichetti ${ }^{3}$, \\ L. Bracci ${ }^{2}$ and R. Moretti ${ }^{1}$ \\ ${ }^{1}$ Division of General and Oncologic Surgery, Careggi \\ Regional and University Hospital, Florence, ${ }^{2}$ Department \\ of Molecular Biology, University of Siena, Siena
}

Introduction: Pancreatic cancer has a 5-years survival rate of less than $5 \%$. The only chance of cure is radical surgery, which increases survival up to $20 \%$. Adjuvant chemoradiotherapy have only little impact. Peptides, like Neurotensin (NT), are a promising class of targeting molecules, due to the presence of overexpressed receptors on the tumoral cells surface. Our previous studies demonstrated how fast degradation of NT carried out by peptidases was prevented by synthesis in a Tetra-Branched-isoform (NT4). NT4 was also conjugated to chemothepeutic agents without affecting receptor binding and activity, both in vitro and in mice.

Materials and Methods: Samples of pancreas adenocarcinoma and corresponding healthy tissue were collected from 15 patients undergone surgical resections. The $10 \mu \mathrm{m}$ thick frozen sections were treated with NT4-Fluorescein, after appropriated passages in different mediums. Controls were performed using an unrelated Fluorescein-conjugated tetra-branched peptide. Peptide binding was analyzed by confocal laser microscope. The images were processed using the ImageJ software. Resulting electronic data were reported as pixel distribution (mean) in the green color range of the RGB system. Wilcoxon rank test for paired samples was used to compare fluorescence signals in healthy and tumor tissues.
Results: When treated with the fluorophore-conjugated branched peptides, all pancreas adenocarcinoma tumor tissues showed remarkably higher fluorescence emission compared to normal tissues from the same patients $(\mathrm{p}<0.001)$.

Conclusions: NT4 analogues represent a novel class of targeting molecules. They have a promising potential for both diagnosis and therapy of pancreatic cancer. Imaging of tumor biopsies might enable pre-treatment estimation of the efficacy of NT-based personalized therapy.

145

\section{Merkel Cell Carcinoma in a Patient with Non-Hodgkin Lymphoma: A Case Report}

\author{
M. Bomboi, M. Pasqualini
}

General Surgery Unit, Ospedale Orlandi, Bussolengo

(Verona), Italy

Introduction: Merkel cell carcinoma (MCC) is an uncommon, neuroendocrine skin tumour with an aggressive clinical course. The etiology of the disease is unknown, although sun exposure and immunosuppression may play a role in its development. Skin cancer and non-Hodgkin lymphoma (NHL) is a relatively frequent reported association in biomedical literature. The aim of this work is to emphasize an unusual clinical presentation (left buttock) of MCC in a patient with lymphoplasmocitic NHL, reflecting about his past and present medical history.

Case presentation: We report a 62-year-old man, suffering from NHL prior treated with chemoterapy and immunotherapy, who presented with a light-red lump on the left buttock that was initially thought to be a skin involvement of the NHL. An excisional biopsy of the mass was performed revealing a MCC (positive immunohistochemical staining positive for CK-20 and negative for TTF-1). Tumour cell were found at $2 \mathrm{~mm}$ from the deep resection margin. A wide excision was performed in association with sentinel lymphnode biopsy (SLNB) from left groin region which resulted positive for MCC micrometastasis. Therefore we carry out a groin lymphnode dissection that revealed metastasis in 2 of 10 lymphnodes. No other metastatic tumours found after a thorough work-up. The patient was treated with local irradiation.

Conclusion: MCC, in this unusual presentation, may suggest a link with NHL and/or more likely with immunosuppressive therapy. 


\section{6}

\section{Right Hemicolectomy for Malignant Neoplasms by Single Port Laparoscopic Technique}

\section{E. Cardone, P. Delrio, F. Ruffolo, F. Bianco, S. De Franciscis, \\ U. Pace, G. Romano}

Department of Coloproctology, National Institute of Cancers "G.Pascale", Naples

Introduction: Single port laparoscopic surgery (SPL) is a rapidly spreading surgical innovation offering mainly cosmetic advantages but also a quicker recovery after laparoscopic abdominal surgery.

Material and Methods: We here describe our initial experience with SPL colorectal surgery in three cases of right colon neoplasms treated by a transumbilical single port access laparoscopic right hemicolectomy. We performed the operation by a single incision laparoscopic device with a multichannel system placed throughout a short umbilical surgical incision.

Results: Three transumbilical right hemicolectomies were performed, respecting oncologic surgical principles of no touch technique with adequate surgical margin and lymphectomy . Pathological specimens were extracted through the umbilical incision and extracorporeal ileocolic anastomosis were performed. Operative time was 85-115 minutes, the length of incision was 5 $\mathrm{cm}$. There were no intraoperative and postoperative complications.

Conclusion: SPL right hemicolectomy is feasible and safe also in patients with malignant lesions. We point out three crucial issues : traditional laparoscopic instruments can be easily used; extraction of the specimen through the umbilical incision allows a good exposure of intestinal loops and extracorporeal ileocolic anastomosis can be easily fashioned, oncological radical resection is achieved. Cosmetic results are obviously excellent and the low rate of abdominal wall complications leads to fast recovery, early return to active life and prompt start of adjuvant treatments if needed.

\section{7 \\ Multidisciplinary Osteo-Oncology Center in the Management of Patients with Bone Metastases}

T. Ibrahim, L. Mercatali, E. Sacanna, P. Serra, R. Ricci,

L. Fabbri, M. Ricci, M.C. Falasconi, R. Casadei, D. Oboldi,

S. Severi, S. Micheletti, E. Petrella, M. Gaudio,

R. Nunziatini, M. Ceccolini, C. Zerotti, D. Cavaliere,

D. Amadori

Osteo-Oncology Center, Istituto Scientifico Romagnolo per lo Studio e la Cura dei Tumori, Meldola, Italy

Introduction: Bone metastases (BM) are responsible for high morbidity in cancer patients. In January 2005 we founded a multidisciplinary Osteo-Oncology Center comprising 17 dedi- cated experts, offering much needed specialist care for patients with BM.

Materials and Methods: The primary objectives of the Center are patient care, $\mathrm{BM}$ research, and specialist training for healthcare workers. By April 2009 our team of experts in oncology, palliative care, orthopedics, radiotherapy, physiatrics, nuclear medicine and radiology had seen 669 patients and carried out 332 monodisciplinary and 980 multidisciplinary visits.

Results: Fourty five percent of patients had breast cancer, $15 \%$ lung, $7 \%$ prostate, $3 \%$ colon, $2 \%$ stomach, $2 \%$ bladder, and $26 \%$ other cancers. High-risk lesions, uncontrolled pain, and first or uncertain BM diagnosis were the main reasons for referral. Sixty-eight percent of patients reported pain, with a median intensity score of 4.04. As a result, analgesic therapy was modified in $42 \%$ of cases. We prescribed radiotherapy, radiometabolic therapy, radiology interventions, orthopedic surgery and orthopedic aids in 223 patients.

Conclusion: Although patient outcome and full economic impact of this new organizational model still have to be analyzed, the high level of patient satisfaction confirms the usefulness of this multidisciplinary approach to the treatment of BM.

\section{8}

\section{Successful Combination of Systemic Therapy with Imatinib Mesilate and Surgery in a Case of Locally Advanced Gastrointestinal Stromal Tumor (GIST)}

D. Cavaliere, F. D'Acapito, D. Dipietrantonio, M. Framarini, F. Tauceri, A. Vagliasindi, G. Solfrini, G.M. Verdecchia

U.O. Chirurgia e Terapie Oncologiche Avanzate Department of Surgery, "GB. Morgagni-C. Forlanini" Hospital, Forlì - Italy

We report a case of a giant GIST of the stomach, curatively resected after neoadjuvant treatment with imatinib mesilate (IM) for 12 months. The patient was a 78-year-old woman who presented at our department with a huge abdominal $(22 \mathrm{~cm}$ in diameter), well-delineated, heterogeneous and exophytic mass. The gastric origin was suspected by imaging and gastroscopy but confirmed only after endoscopic ultrasonography. Histopathologic assessment of fine needle biopsy revealed a c-kit positive GIST with hight risk. Morphological and functional imaging methods were used for staging: computed tomodensitometry (CT) and positron emission tomography using [(18)F]-2-fluoro-2-deoxy-D-glucose (FDG), did not show any metastatic spreading of the disease but only a locally advanced GIST. Nevertheless, radical operation was considered difficult at that point. Therefor IM was administered as neoadjuvant therapy ( $400 \mathrm{mg} /$ day) according to the NCCN Guidelines. A sensible decrease in FDG uptake was early detected during systemic therapy: from a standard uptake value (SUV) of 15.38 at the base-line examination to a SUV of 3.9 twenty days following therapy initiation, confirming the early response to IM regimen. After 10 months of treatment, CT revealed a maximal reduction in tumor diameter (about 30\%) as well as modification in tumor density and enhancement due to the fibromyxoid 
transformation induced by IM therapy. The radical operation was considered feasible and programmed before the development of secondary resistance. Radical surgery was performed without tumor disruption and organ mutilation: the tumor invaded adjacent transverse mesocolon but not other structures. A wedge gastric resection with en-bloc extirpation of the abdominal mass and transverse mesocolon was performed. The postoperative course was uneventfull. Adjuvant therapy with IM (400 mg/day for 12 months) was indicated by a high risk behaviour. The patient was recurrence free at her 9-month follow-up examination.

Conclusions: This case confirm that locally advanced GIST can be advantageously treated with IM as neoadjuvant. The preoperative therapy can facilitate resection and avoid mutilating surgery by decreasing tumour size, risk of tumor disruption and hemorrage. This case also highlights that combination of systemic therapy and surgery can be safely performed in older patients when their performance status and comorbidities are carefully considered by an experienced multidisciplinary team.

\section{9}

\section{Oncologic and Functional Outcome of Robot-Assisted Rectal Surgery}

\section{Ceribelli, A. Patriti, G. Ceccarelli, A. Bartoli, L. Casciola}

Department of General, Minimally Invasive and Robotic Surgery, San Matteo degli Infermi Hospital, Spoleto

Background: Traditional Laparoscopic anterior rectal resection has been recently used for rectal cancer offering good functional results compared with open anterior resection and resulting in a better post-operative early outcome. Few studies investigating the role of robot-assisted rectal surgery (RARS) have been carried out showing its feasibility. The aim of the study was to verify the perioperative and oncologic outcome of RARS.

Methods: Thirty-three patients consecutively undergoing RARS were reviewed.

Data of the patients were collected prospectively and analyzed in order to determine intraoperative, oncologic and functional outcome.

Results: A total of 31 restorative surgeries and 4 abdominal-perineal resections were carried out. No conversions were recorded, mean blood-loss $224 \pm 285 \mathrm{ml}$ (1 intraoperative transfusion) and the mean operative time was $250 \pm 72$ minutes. The number of harvested nodes $(11 \pm 6)$ and margin status compared favorably with those of the open series, the radial margin was negative in all patients. Post-operative sexual impairment was present in $6 \%$ of patients. No local recurrences occurred during the follow-up period.

Conclusion: RARS is a safe and feasible procedure that may facilitate mesorectal excision. Randomized clinical trials and longer follow-ups are needed to evaluate a possible influence of RARS on patient survival.
150

\section{Case-Matched Analysis of Totally Laparoscopic Versus Open Liver Resection for HCC: Short and Middle Term Results}

\author{
F. Cipriani, F. Ratti, E. Guzzetti, M. Catena, M. Paganelli, \\ L. Aldrighetti
}

Department of Surgery_Liver Unit, Scientific Institute H San Raffaele, Vita-Salute San Raffaele University, Milan, Italy

Introduction: Laparoscopy is gaining acceptance as a safe procedure for resection of liver neoplasm. The aim of this study is to evaluate surgical results and mid-term survival of minor hepatic resection performed for HCC.

Material and Methods: Data of 25 patients with HCC undergoing laparoscopic hepatectomy from September 2005 to February 2010, were compared to a control group of 25 patients who underwent open resection (OR) in the same period. The two groups were matched in terms of type of resection, tumor size, severity of cirrhosis and age of patients.

Results: Three patients underwent conversion to an open approach. Laparoscopic approach resulted in shorter operating time (175 min, p 0.038), lower blood loss ( $253 \mathrm{~mL}, \mathrm{p} 0.004)$ and lower blood transfusion rate $(14.8 \%, \mathrm{p} 0.037)$. There was no difference in perioperative morbidity and mortality rate; laparoscopic approach was associated to a shorter hospital stay (7 days, $\mathrm{p}$ 0.042). After a mean follow up of 32 months, disease free survival and overall survival were 42.7 and 27.6 months for laparoscopic group, and 47.7 and 31.4 months for OR group (p NS).

Conclusion: Laparoscopic resection of HCC is feasible and safe in selected patients, and can result in good surgical results, with similar outcomes in terms of overall and disease-free survival.

151

\section{Accuracy of Multi Slices Computed Tomography (MSCT) Scan in Preoperative Staging of Para-aortic Lymph Nodes in Patients with Gastric Cancer. A Prospective Single-Center Study}

\author{
M. Di Martino, D. Marrelli, M.A. Mazzei, P. Bambacaro*, \\ C. Pedrazzani, G. Corso, S. Caruso, E. Morelli, \\ L. Volterrani, F. Roviello, E. Pinto \\ Department of Human Pathology and Oncology, Section \\ of Surgical Oncology, University of Siena, Italy, \\ *Department of Human Pathology and Oncology, Section \\ of Radiology, University of Siena, Italy
}

Aim: To analyze the diagnostic accuracy of MSCT scan in the identification of para-aortic lymph node metastasis from gastric cancer.

Design of the Study: Prospective single-center. 
Material and Methods: 92 consecutive patients with endoscopic diagnosis of gastric cancer were submitted to preoperative MSCT staging according to a common protocol in the period 2001-2009. All diagnostic procedure were performed by dedicated radiologists who were unaware of the final pathological nodal status.

Subsequently all patients underwent potentially curative resection (R0) with super-extended (D3) lymphadenectomy according to the criteria described by Japanese Research Society for Gastric Cancer. Lymph nodal mapping in different stations and retrieval of single lymph nodes were performed by the surgeon on the fresh specimen, and then submitted for pathological examination. Clinical, radiological and pathological data were prospectively stored on database.

Results: In 13 out of 92 included patients (14\%), pathological examination demonstrated the involvement of para-aortic lymph nodes; in 11 of these cases, MSCT scan successfully identified metastases (sensitivity: 85\%). In 79 patients no pathological para-aortic involvement was observed; for these patients, MSCT resulted correctly negative in 75 cases (specificity: 95\%). Positive (PPV) and negative (NPV) predictive values resulted $73 \%$ and $97 \%$, with a global accuracy of $93 \%$.

Conclusion: MSCT scan performed according to a common protocol by dedicated radiologists demonstrated high accuracy in preoperative identification of para-aortic nodal metastases from gastric cancer. These results may be useful in planning surgical approach for gastric cancer patients.

\section{2}

\section{Solid Pseudopapillary Tumor of the Pancreas in Children}

\author{
P.M.C. Tomaiuolo, A. Mazzari, U. Grossi, P. Giustacchini, \\ A. Crucitti, R. Bellantone \\ Department of Surgery, Catholic University of Rome
}

Introduction: Solid pseudopapillary tumor (SPT) is rare, accounting for up $0.13-2.7 \%$ of all pancreatic neoplasm. It was first reported by Lichtenstein in 1933 and fatherly described in 1959 by Frantz et al. To date, near 1.000 cases have been documented in the English Literature, more than two third of which have been reported in the past ten years. SPTs are uncommon in children, representing about $8 \%$ of all cases, and show different clinical features compared to adults. Surgical resection is the mainstay of therapy, reaching more than $95 \%$ overall 5 -ys survival. A pediatric case is here reported.

Materials and Methods: A 15-years-old girl with a $12 \times 14 \times 10 \mathrm{~cm}$ solid mass growing from the tail and the body of the pancreas, involving spleen, left adrenal gland and kidney, stomach and some bowel loops, was referred for surgical treatment. A distal pancreatectomy with splenectomy was performed.

Results: On histopathological examination the tumor was a $14 \mathrm{~cm}$ well-circumscribed solid mass, with pseudopapillary cell architecture, showing strong cellular immunoreactivity for alpha-1 antitrypsin, vimentin, neurone-specific enolase, progesterone receptors and in part to CD10 and CAM 5.2, but not to sinaptofisin and chromogranin. The patient had an uneventful post-operative course and was discharged on the 13th postoperative day. A thirteen-months post-surgical follow-up after successful surgical resection showed no evidence of recurrent disease.

Conclusion: Solid pseudopapillary tumors show different clinical features in the pediatric age group. High long-term survival rates can be achieved in most cases, warranting aggressive treatments even in metastatic disease.

\section{3}

\section{Predictive Factors of Tumor Response and Survival in Locally Advanced Rectal Cancer Treated with Neoadjuvant Chemoradiation}

\author{
A. Mazzari, U. Grossi, P.M.C. Tomaiuolo', \\ M.A. Gambacorta§, B. Barbaro*, V. Valentini`, A. Crucitti, \\ R. Bellantone
}

Department of Surgery, ${ }^{\S}$ Department of Radiotherapy, *Department of Radiology Catholic University of Rome

Introduction: Aim of this study is to investigate the predictive role of clinical, biological and pathological markers on tumor response and survival in locally advanced rectal cancer (LARC) treated with neoadjuvant chemoradiotherapy (CRT).

Materials and Methods: From January $1^{\text {st }} 2000$ to February $28^{\text {st }} 2009,78$ consecutive patients, affected by LARC, underwent preoperative CRT followed by surgery. Age, gender, ASA score, pre-treatment serum carcinoembryonic antigen (CEA $\leq 4$ vs $>4$ $\mathrm{ng} / \mathrm{ml}$ ), interval between completion of CRT and surgery ( $\leq 9$ vs $>9$ weeks), tumor regression grade (TRG), pathological complete response (pCR) and long-term survival were evaluated. Variables were analyzed by univariate and multivariate analysis; $p<0.05$ was considered statistically significant.

Results: Twenty patients (25.6\%) achieved a pathological complete response ( $\mathrm{pCR}$ ). Higher $\mathrm{pCR}$ rate was found in patients with lower pre-CRT CEA levels $(p=0.019)$. On multivariate analysis, an interval $>9$ weeks between treatment completion and surgical resection $(p=0.043)$ as well as pre-CRT CEA levels $\leq 4 \mathrm{ng} / \mathrm{ml}$ ( $p=0.039)$ were significantly associated with a higher rate of nodal downstaging, which correlated with improved overall survival and decreasing local recurrence.

Conclusion: Our data suggest that pre-CRT serum CEA levels $<4 \mathrm{ng} / \mathrm{ml}$ could play a prognostic role in predicting tumor response. Improved long-term survival rates can be achieved by increasing the interval between completion of CRT and surgery. 
154

\section{"Rank/Rankl Expression: A Possible Prognostic Factor of Gastric Cancer"}

F. Longo, G.T. Capolupo, A. Coppola, I. Cipollone, A. Calmieri, D. Santini, P.Crucitti, R.Coppola

General surgery Department Campus Bio-medico Rome, Clinical Oncology Department Campus Bio-medico Rome

Introduction: Enhanced production of RANKL and its binding to RANK on the osteoclasts have been associated with osteolysis in breast and prostate cancer bone metastasis. In 2006 Hiroky Kusumoto et all. pointed out a possible correlation between RANK expression on gastric cancer cells surface and the onset of distal bone metastases. With the present study we tried to evaluate the role of RANK/RANKL overexpression in patients who underwent R0 gastric resection for carcinoma in terms of DFS and OS.

Materials and Methods: We enrolled 65 patients who underwent gastric resection for adenocarcinoma from September 1999 to December 2008. We retrieved the operatory inclusions and for each block specific immunohistochemical reaction for RANK was performed using monoclonal mouse antibodies RANK/ TNFRSF11A. The extent of immunohistochemical reaction was evaluated by light microscopy at 4 degrees of staining. We then correlated by statistical analysis the immunohistochemical positivity for RANK with the following parameters: sex, histological type, T, N, mucinos histologic subtype vs others, DFS and OS.

Conclusions: RANK/RANKL pathway may influence the natural history of gastric cancer. Although RANK overexpression is not correlated with OS, it seems to lead to a more invasive loco-regional desease in terms of $\mathrm{T}$ and lymphnode involvement. Moreover, RANK overexpression in gastric carcinomas with predominantly mucinous extracellular seems very interesting. Further investigations in this direction could have important clinical and therapeutic implications.

155

Phase II Evaluation of ${ }^{90}$ Yttrium Resin Microspheres (SIR-Spheres) Monotherapy in Unresectable, Heavily Pre-treated Colorectal Liver Metastases

R. Mancini', L. Carpanese1, R. Sciuto', R. Golfieri², E. Giampalma², F. Izzo ${ }^{3}$, F. Fiore ${ }^{3}$, P. Cagol'4, S. Bacchetti ${ }^{4}$,

C.L. Maini ${ }^{1}$, G. Pattaro ${ }^{1}$, M. Cosimelli ${ }^{1}$, on behalf of the Ita lian Society of Locoregional Therapy in Oncology,

(S.I.T.I.L.O.) Group

${ }^{1}$ Regina Elena National Cancer Institute, Rome, Italy, ${ }^{2}$ University of Bologna, Italy, ${ }^{3}$ Cancer Institute of Naples, Italy, ${ }^{4}$ University of Udine, Italy

Introduction: This multicentre phase II study is the first prospective evaluation of selective internal radiation therapy (SIRT) as salvage therapy for patients with colorectal liver metastases progressing after oxaliplatin- and irinotecan chemotherapy.
Methods: Fifty patients with adequate hepatic function were eligible for inclusion in absence of major vascular anomalies and pulmonary shunt $<10 \%$. The median follow-up was 11 months. Thirty-eight (76\%) had already received at least 4 lines of chemotherapy. Most patients had synchronous disease (72\%), $>4$ hepatic metastases (58\%), 25-50\% liver involvement (60\%) and bilateral spread (70\%).

Results: Early and late ( $>48$ hrs) WHO G1-2 toxicity was 16\% and $22 \%$, respectively. Forty-six pts were evaluable for response under RECIST criteria. One (2\%) had complete response (CR), 11 (22\%) partial response (PR), 12 (24\%) stable disease (SD) and 22 (44\%) progressive disease (PD). The median overall survival was 13 months, 16 months in the responders $(\mathrm{CR}+\mathrm{PR}+\mathrm{SD})$ and $8 \mathrm{mos}$. in non-responders (PD) ( $p=0.0006)$. Two-year survival was $40.3 \%$ and $0 \%$ respectively. Median time to progression was 4 months. Various molecular markers among responders were evaluated and presented below.

Conclusions: In the heavily pretreated pts, ${ }^{90}$ yttrium microspheres produced an encouraging median survival, with acceptable toxicity, that compares favourably with phase II/III studies of chemotherapy used as second- or subsequent lines of therapy. Clinical response often correlates with a more favourable biological profile of lesions after SIRT.

\section{6}

\section{Local Excision in Locally Advanced Rectal Cancer: Is a Safe Alternative Treatment Option?}

R. Mancini', M. d'Annibale', F. Ambesi-Impiombato², C. Garufi', M. Zeuli3, M. Caterino4 ${ }^{4}$, I. Sperduti', G. Pattaro', M. Cosimelli

${ }^{1}$ Dept. of Surgery, ${ }^{2}$ Div. of Radiotherapy, ${ }^{3}$ Dept. of Oncology, ${ }^{4}$ Div. of Radiology, ${ }^{5}$ Unit of Statistics, "Regina Elena" Cancer Institute of Rome, Italy

\section{3rd Classified - SPIGC Award Session, Surgical Oncology}

Introduction: Aim of this prospective study was to evaluate feasibility, safety and oncological outcomes in cT3-4 rectal cancer patients submitted to Local Excision after clinical complete response to preoperative radiochemotherapy.

Materials and Methods: From 2004 to 2006, 24 rectal cancer pts. staged T3-4 were submitted to radiochemotherapy followed by local excision after clinical complete response. Staging and restaging were carried out by digital examination, endoscopy, endorectal ultrasound and CT scan.

Treatment toxicity, surgical morbidity, local recurrence, overall and disease-free survival after a minimum follow-up of 39 mos were evauated.

Results: The GII-III toxicity was $33.8 \%$, surgical morbidity $4.1 \%$, pathological complete response was observed in 13 pts. (54.2\%), ypT1-2 were 10 pts. (41.6\%), 1 pts. showed mesorectal cancer residual microfoci. Local recurrence was $20.8 \%, 80 \%$ were endoluminal, restorative TME was possible in all pts. and all are 
actually disease-free. The 5-yr. overall and disease-free survival were 94 and $68 \%$ respectively.

Conclusions: Local Excision is a safe alternative to conventional resection in strictly selected patients with complete response confirmed by pathological assessment. Randomized studies are needed. LE after chemoradiation is a safely alternative in pts. with major co-morbidity or refusing stoma.

\section{7}

Phase II Evaluation of ${ }^{90}$ Yttrium Resin Microspheres (SIR-Spheres) in Unresectable, Heavily Pre-treated Colorectal Liver Metastases

\author{
R. Mancini ${ }^{1}$, L. Carpanese 1, R. Sciuto ${ }^{1}$, R. Golfieri², \\ E. Giampalma', F. Izzo ${ }^{3}$, F. Fiore ${ }^{3}$, P. Cagol', S. Bacchetti ${ }^{4}$, \\ C.L. Maini ${ }^{1}$, G. Pattaro ${ }^{1}$, M. Cosimelli ${ }^{1}$ on behalf of the Itali \\ an Society of Locoregional Therapy in Oncology, \\ (S.I.T.I.L.O.) Group \\ ${ }^{1}$ Regina Elena National Cancer Institute, Rome, Italy, \\ ${ }^{2}$ University of Bologna, Italy, ${ }^{3}$ Cancer Institute of Naples, \\ Italy, ${ }^{4}$ University of Udine, Italy
}

Introduction: This is the first prospective multicentre phase II study to evaluate selective internal radiation therapy (SIRT) as salvage therapy for patients with colorectal liver metastases progressing after oxaliplatin- and irinotecan chemotherapy.

Methods: Fifty patients with absence of vascular anomalies and pulmonary shunt $<10 \%$ were included. The median followup was 11 months. Most patients had synchronous disease (72\%), $>4$ hepatic metastases (58\%), 25-50\% liver involvement (60\%) and bilateral spread (70\%).

Results: Early and late ( $>48$ hrs) WHO G1-2 toxicity was $16 \%$ and $22 \%$, respectively. Forty-six pts were evaluable for response under RECIST criteria. One (2\%) had complete response (CR), 11 (22\%) partial response (PR), 12 (24\%) stable disease (SD) and $22(44 \%)$ progressive disease (PD). The median overall survival was 13 months, 16 months in the responders and 8 mos. in non-responders (PD) ( $\mathrm{p}=0.0006)$. Two-year survival was $40.3 \%$ and $0 \%$ respectively. Median time to progression was 4 months. Various unfavourable molecular markers evaluated (Ki-67>50\%, p-53 +, Survivin) were underexpressed in the responders posttreatment.

Conclusions: In the heavily pretreated pts, ${ }^{90}$ yttrium microspheres produced an encouraging median survival, with acceptable toxicity, that compares favourably with phase II/III studies of chemotherapy used as second- or subsequent lines of therapy. Clinical response often correlates with a more favourable biological profile of lesions after SIRT.

\section{8}

\section{Monotrocar Laparoscopy for Minitinvasive Preoperatory Stadiation of Tumor and Second Look}

\author{
P. Marsanic, B. Mussa, A. Mussa* \\ ${ }^{*}$ Chirurgia Oncologica Prof.Mussa Osp. San Giovanni \\ Battista, Torino
}

Introduction: Nowaday preoperative cancer's stadiation of is primary to settle timing of multimodal therapy. It is also very important restadiation of ovaric cancer by second look and it will be even important for other abdominal neoplasies. Laparoscopy is very useful for this purpose because it allows to inspect the whole abdominal cavity and to biopsy. It is both diagnostic and therapeutic. Monotrocar laparoscopy allows to inspect the whole abdominal cavity and to perfom surgery independently from trocar's position and with a minor trauma than traditional laparoscopy. So this approach could be a better method for stadiation of cancer before surgery and in follow up.

Materials and Methods: From March 2009 to decemeber 2009 three patient were operated with monotrocar laparoscopy . These patients were affected respectively of ovaric cancer, ileal limphoma and carcinomatosis from unknown tumor. In the first patient we used monotrocar laparoscopy for second look. In the second patient we used monotrocar laparoscopy to stadiate the neoplasy and for neoplasy's excission because it was stenosing. In the last patient we used monotrocar laparoscopy as diagnostic method to have histology and to stadiate the cancer.

Results: In our little experience this method hadn't complications and permetted a lower postoperative pain and an early healing and an early beginning of chemotherapy than traditional surgery. We didn't observe metastasis in trocar site.

Conclusions: Monotrocar laparoscopy finds best application as diagnostic and sometimes therapeutical method in oncological surgery with cost and morbility reduction.

\section{9 \\ Dworak Grade in the Cancer Rectum with Preoperative Radiochemiotherapy: Follow- up to 5 and 10 Years}
A. Mauro, M. Gavioli, A. Schiavone, A. Marazzi, G. Natalini

Introduction: Preoperative radiochemotherapy reduces tumor size and nodal metastasis in patients with rectal cancer. Tumor downstaging has been associated with an increased probability of a sphincter-saving procedure and with improved local control. The complete sterilized and the minimal residual disease are considered similar. We evaluated the incidence of these two conditions and analyzed their impact in terms of local recurrence, distant metastasis, and survival, we studied the prognostic value of grade of regression (GR) 
Material and Methods: We studied 115 uT3/T4N0/N+ rectal cancers, treated with preoperative radiochemotherapy and curative surgery after four to eight weeks, from June 1999 to July 2004 (65 males, mean age: 64 years).

We evaluated ypTNM stage and tumoral regression, according to the five degrees proposed by Dworak.

Results: Tumor down-staging occurred in 77 (67\%) patients, including $25(21,7 \%)$ patients with pathologic complete response, 25 patients with GR3, 30 with GR2, 32 patients with GR1 and 3 GR0.

In median follow-up of 105 months, the percentage of local recurrence was $3.4 \%$ percent, distant recurrence $17.4 \%$, local and distant recurrence $3.4 \%$; 82.6 percent of patients were alive.

The disease-free survival after 8 years for patients with GR4, GR3, GR2, GR1, GR0 was 96\%, 84\%, 70\%, 65.7\%, 33\%, respectively. The difference between five groups, in terms of local recurrence, distant metastasis, and survival is statistically significant.

Conclusions: The complete pathologic response, after preoperative therapy, shown an excellent prognosis. The minimal residual disease has a much better prognosis in comparison with the gross residual disease.

160

\section{Prognostic Factors and Oncologic Outcome in 146 Patients with Colorectal Peritoneal Carcinomatosis Treated with Cytoreductive Surgery Combined with Hyperthermic Intraperitoneal Chemotherapy: Italian Multicenter Study S.I.T.I.L.O}

\begin{abstract}
I. Monsellato1, F. Cavaliere1, M. De Simone', S. Virzi”, M. Deraco ${ }^{4}$, C.R. Rossi ${ }^{5}$, A. Garofalo6, F. Di Filippo, M. Vaira ${ }^{2}$, M. Valle ${ }^{6}$, P. Pilati ${ }^{5}$, P. Perri, ${ }^{6}$ M.L. Pinta', R. D'Alessandro7, D. Giannarelli"
\end{abstract}

'Department of Surgery, San Giovanni Hospital, Rome, ${ }^{2}$ Department of Surgery, Surgical Oncology Unit, San Giuseppe Hospital, Empoli, ${ }^{3}$ General Surgery Unit, Bentivoglio Hospital, Bologna, 'Department of Surgery, Melanoma Sarcoma Unit, National Cancer Institute of Milan, ${ }^{5}$ Department of Oncological and Surgical Sciences, University of Padova, Padova, ${ }^{6}$ Surgical Oncology, Regina Elena National Cancer Institute, Rome, ${ }^{7}$ Dept. Of Laboratory Medicine and Advanced Biotechnology, IRCCS san Raffaele "La Pisana", Roma, ${ }^{8}$ Biostatistical Unit,

Regina Elena National Cancer Institute, Rome

Introduction: Peritonectomy procedures combined with hyperthermic intraperitoneal chemotherapy have altered the history of colorectal peritoneal carcinomatosis allowing a consistent long-term survival. In this study we assessed major clinical-pathological variables to investigate whether the currently used criteria appropriately select patients to undergo locoregional cytoreductive surgery combined with HIPEC.

Materials and Methods: Preoperative, operative and follow-up data on 146 consecutive patients presenting with colorectal peritoneal carcinomatosis and treated by surgical cytoreduction combined with HIPEC in 5 Italian Hospital and University centers were prospectively entered in a database. Univariate and multivariate analyses were used to assess the prognostic value of clinical and pathologic factors.

Results: Over a minimum 24 month-follow-up, overall morbidity rate was $27.4 \%$ directly related to the extent of surgery. Peritoneal cancer index, unfavorable peritoneal sites, synchronous or previously resected liver metastasis and the completeness of cytoreduction, all emerged as independent prognostic factor correlated with survival.

Conclusions: Until more effective criteria based upon biomolecular features of carcinomatosis are investigated, patients should be selected according to the existing independent prognostic variables.

\section{1}

\section{Role of Thermoablation of Kidney Tumors in Patients Not Eligible for Surgery Treatment}

\author{
R.F. Grasso, M. Nezzo, C. Mallio, E. Faiella, G. Luppi, \\ B.B. Zobel \\ Chirurgia Oncologica
}

We performed radiofrequency ablation (RFA) on 33 patients (11, 220", from 21 to $88 \mathrm{y}$ ), with solid renal tumors. Included patients were not eligible for surgical treatment because of severe comorbidities, solitary kidney patients, bilateral renal cell carcinoma or von Hippel-Lindau syndrome. The diameter of the lesions ranges from $0.8 \mathrm{~cm}$ to $7 \mathrm{~cm}$ (mean $1.9 \mathrm{~cm}$ ); we had $79 \%$ exophytic growth vs $21 \%$ of growth towards the renal hilum. We used in 4 cases a RITA generator with hook needle, in 29 cases a Tyco Radionics (Valleylab) generator, 200W with water-cooled needle. Follow-up ranges between 1 month and 3 years (mean 9.7 months). Of the 30 tumors $<4 \mathrm{~cm}$ in 29 cases we found a complete ablation at the first procedure, while in 1 case there was local persistence at 1 month. In 3 lesions $>4 \mathrm{~cm}$, the ablation was combined with embolization. We had complications in 2 treatments (6\%): a perinephric hematoma reabsorbed spontaneously (minor complication); a ureteral post-treatment stenosis of the uretheropelvic junction (major complication). RFA with a good selection of patients can really treat the solid tumors of the kidney with a small percentage of complications. After the first procedure we had the best results treating lesions $<4 \mathrm{~cm}$, with no residual tumor. For lesions $>4 \mathrm{~cm}$ we prefer to associate the embolization to definitively treat the whole tumor. 


\section{2}

Computed Tomography and US-Guided Core Needle Biopsy for Bone Lesions

\author{
R.F. Grasso, M. Nezzo, G. Cazzato, E. Faiella, G. Luppi, \\ B.B. Zobel \\ Chirurgia Oncologica
}

Imaging-guided core needle biopsy is a well-established technique for the diagnosis of primary bone tumors, metastases or infectious diseases. The biopsies were US or CT guided. 77 biopsies were performed on 69 patients and histologically examined. The age ranged from 15 to 83 years (mean 59 years). To assess the accuracy of the procedure, we compared the diagnosis at biopsy with the diagnosis after definitive surgery (when available). Bone core needle biopsy showed malign tumors in 40 patients (58\%), and in 22 patients (31\%) benign tumors or infectious diseases. In 7 patients the result was inconclusive. Within those 7 patients we needed to repeat the procedure once and in one case twice to reach the diagnosis. The core needle biopsy histologic diagnosis was compared with that of the definitive surgery and the diagnostic accuracy was $92 \%$. We had just two minor complications during the procedures. CT or US-guided bone biopsy is a safe and effective procedure that assures sufficient and proper material for histologic examination. Tumor sampling is extremely important, and cores should be taken in different directions, including areas of necrosis. The processing is quick, and diagnosis can be achieved within 24 hours.

\section{3 \\ Our Clinical Experience on 16 Patients with Lung Tumors Treated with Percutaneous Radiofrequency Thermal Ablation (RFA) \\ G. Luppi, E. Faiella, M. Nezzo, F. Carcione, R.F. Grasso, B. B. Zobel \\ Chirurgia Oncologica}

Radiofrequency ablation (RFA) may be an alternative to surgery or radiation therapy to eliminate primary lung cancer lesions or pulmonary metastases. RFA destroys the entire lesions using heat to kill the malignant cells in a minimally invasive way, achieving and maintaining a $50-100^{\circ} \mathrm{C}$ temperature range throughout the entire volume of the target tumor. Patients eligible for RFA must not be surgical candidates. To achieve complete ablation we must ablate a peripheral margin of $0.5-1 \mathrm{~cm}$ of normal tissue surrounding the tumor, as well as the entire tumor itself. The procedure is US or CT-guided. We performed 16 RFA, 5 primary lung malignant lesions (31\%) and 11 metastases (69\%);90" (56\%), $7 \%$ (44\%), from 57 to 88 years (mean $72 y$ ). The diameter of the lesions ranges from $7 \mathrm{~mm}$ to $51 \mathrm{~mm}$ (mean $19 \mathrm{~mm}$ ). Treating large tumors or lesions located adjacent to critical structures it is not prohibitive. Pneumothorax is a common complication. Post-procedural pleurisy and small pleural effusions are observed mainly in patients with pleural-based and peripheral lesions, but thoracen- tesis is usually not required, because these lesions are self-limited. We didn't have major complications. The 1 and 6 months followup DWI MR or CT guided demonstrated just one recurrence in a metastatic lesion. Thus RFA has a significant role in the treatment of unresectable lung tumors, prolonging patient survival.

\section{4 \\ Outcome of Hepatic Resection at a Low Volume Center}

C. Di Somma, D. Pertile, N. Solari, F. Cafiero

Surgical Oncology Department, Epatobiliopancreatic Surgery, IST - National Cancer Research Institute, Genova - Italy

Introduction: Several studies support the hypothesis that hepatic resection could have better outcome if performed at high volume center. We analise the experience of National Cancer Research Institute of Genova to evaluate the adequacy of morbidity and mortality rates comparing our results with those of the most important series in Literature.

Material and Methods: From Jenuary 2002 to March 2010, 70 patient were subjected to an hepatic resection in our structure (8,4 patients/year). The average age was 63,3 year old .46 patients had comorbidity, mainly cardiovascular. 36 patients underwent a major hepatectomy. In 20 patient liver resection was synchronous with other abdominal resection. 2 patient required a common bile duct resection with a bilioenteric anastomosis.

Results: Postoperative Mortality rate was $1,4 \%$ (1 patient). Overall morbidity was $31,0 \%$ : all complication were treated conservatively. According to studies with largest number present in Literature, morbidity and mortality rate range from 0,55 to $4,9 \%$ and from 16,4 to $45 \%$ respectively.

Conclusion: Our data suggest that liver resection can be performed safely also at a low volume center: a trained surgical equipe, anestesiologist and nursing staff experienced in managing patients in complex conditions are necessary, with availability of an Intensive Care Unit.

\section{5}

\section{A Rare Pancreatic Metastasis from Melanoma: Case Report \\ O. Giovanni, G. Riccardo, P. Nicola*, M. Luca', S. Raffaella’, B. Paolo \\ Surgical Pathology - Dept. of Medical and Surgical Critical Care, University of Florence, ${ }^{*}$ Dept. of Dermatological \\ Sciences, University of Florence, 'Dept. of Human \\ Pathology and Oncology, University of Florence}

Introduction: Pancreatic metastases represent $2-5 \%$ of the malignant pancreatic neoplasm. The most important cause of pancreatic metastases is Renal Cell Carcinoma (RCC) but are also 
observed lung, breast, colon metastases and exceptionally melanoma metastases.

Materials and Methods: We describe the case of a 42 year old man who had undergone a lymphadenectomy on the right armpit for a metastatic melanoma. Primitive lesion is unknown. The follow up was negative for any lesions up to July 2005 when a total body CT-scan pointed out a single lung lesion, that was removed with a wedge resection of the right lower lobe and biopsy (negative) of mediastinal lymph-nodes. Later he underwent a single chemotherapy cycle with Fotemustine.

The follow up has been uneventful until February 2010 when a total body CT-scan showed a pancreatic metastasis with superior mesenteric vein invasion with no symptoms or any clinical signs.

Results: The patient underwent a Whipple procedure plus thrombectomy of neoplastic invasion of the superior mesenteric vein. The post operative period was uneventful, and the patient was discharged after eleven days of hospital staying. The patient is currently receiving adjuvant chemotherapy.

Conclusions: The indications to a surgical approach for a single pancreatic metastasis from melanoma is uncertain, but in strictly selected patients, it is possible to improve their quality of life.

A discussion of the indications to surgery is provided.

\section{6}

\section{Cases of "Two Stage Hepatectomy" for Liver Metastases from Colorectal Cancer: A Single Centre Experience}

\author{
F. Ratti, F. Cipriani, C. Soldati, M. Catena, M. Venturini, \\ L. Aldrighetti \\ Dipartimento di Chirurgia Generale e Specialistica - Liver \\ Unit. IRCCS H San Raffaele, Università Vita-Salute San \\ Raffaele, Via Olgettina, Milano, Italy
}

Introduction: Most patients with colorectal liver metastases (CLM) present with unresectable disease at diagnosis, while only $15-25 \%$ are eligible for a surgical program. The best strategy to address the issue of non-resectability is the use of a multidisciplinary approach: liver function analysis, modern imaging techniques, liver volumestudy, advanced surgical techniques, portal embolization, new patterns of CT.

Materials and Methods: Between January 2006 and December 2009, 30 patients with multiple bilobar CLM underwent two stage hepatectomy at the Department of Surgery - Liver Unit HSR. In each patient CT volumetric assessment of hepatic volumes was before PVE and after 4 weeks.

Results: The mean volume of the FRL at the time of the procedure and after 4 weeks was respectively $412.7 \mathrm{cc}( \pm 189.56)$ and $573.78 \mathrm{cc}( \pm 180.25)$ with an average increase of $50,7 \%( \pm 32.95)$. The duration of the intervention, the portal clamping, intraoperative blood loss and postoperative hospital stay were lower in the first than the second intervention. It was calculated a morbidity of $12 \%$ and $39 \%$ respectively related to the first and second time surgery. During the postoperative course of the second intervention, only 2 patients showed mild and transient signs of liver failure.
Conclusions: The two-stage hepatectomy provides additional possibilities for treating patients suffering from CLM, although careful selection of patients is required even considering the evident major complexity of the second intervention.

\section{7 \\ Laparoscopic and Open Approach for Synchronous Colorectal Liver Metastases}

F. Ratti, L. Aldrighetti, S. Di Palo, E. Orsenigo, M. Catena,
E. Guzzetti, L. Ghirardelli, C. Staudacher, G. Ferla

Dipartimento di Chirurgia Generale e Specialistica - Liver Unit. IRCCS H San Raffaele, Università Vita-Salute San Raffaele, Via Olgettina, Milano, Italy.

Introduction: Approximately $30 \%$ of patients with colorectal cancer have synchronous liver metastases. The long-term survival for these patients is affected by the achievement of a complete removal (R0) of the primary tumor and liver metastases in a single stage or in two separate operations. Aim of the study was the evaluation of short-term results of combined resection of cancer of the left colon or rectum and liver metastases, comparing the outcome of resection of the primary tumor performed laparoscopically or by laparotomy.

Patients and Methods: From January 2004 to December 2009, 61 patients underwent combined resection of colorectal cancer and synchronous liver metastases. Thirty-two patients underwent laparoscopic colorectal resection (LPS Group), and were compared with 29 patients undergoing colorectal resection via laparotomy (LPT Group). The liver resection was performed by laparotomy. It was performed further analysis, including only the colonic resection.

Results: LPS group was associated with a lower postoperative morbidity ( $25 \%$ vs $51.7 \%, \mathrm{P}=0.031$ ), and reduced postoperative hospital stay ( 10.5 vs 15.1 days, $P=0.002)$. The analysis of the subgroup of colonic resection showed a significant reduction in postoperative morbidity $(18.7 \%$ vs $50 \%, \mathrm{P}=0.049)$ and duration of hospital stay ( 9 vs. 15.2 days, $\mathrm{P}=0.002$ ).

Conclusions: Laparoscopic colorectal resection in patients undergoing simultaneous open liver resection of the metastases is associated with reduced morbidity and postoperative hospital stay. 
168

\section{Treatment of Recurrent Colorectal Liver Metastases: Is Repeat Surgery Safe and Effective? A Single Centre Experience}

F. Ratti, F. Cipriani, M. Catena, M. Paganelli, L. Aldrighetti, G. Ferla

Dipartimento di Chirurgia Generale e Specialistica - Liver Unit. IRCCS H San Raffaele, Università Vita-Salute San

Raffaele, Via Olgettina, Milano, Italy

Introduction: Hepatic resection is the only potentially curative treatment for liver metastases from colorectal cancer (CLM). $60-70 \%$ of patients undergoing surgical treatment develop recurrent disease and about $30 \%$ of recurrences are in the liver. We analyzed intra-and postoperative outcome, recurrence and survival after primary (PR) and repeated (RR) hepatic resection for CLM to determine the value of this approach in patients with recurrent disease.

Materials and Methods: From January 2000 to January 2006, 298 liver resections for CLM in 245 patients were carried out at the Dipartimento di Chirurgia Generale e Specialistica Liver Unit HSR. 53 of these underwent RR and are the object of the study. PR and RR were compared in terms of type of hepatic resection, intraoperative blood loss, postoperative complications, hospital stay and mortality and finally overall survival (OS) and disease-free survival (DFS) were evaluated.

Results: There was no statistically significant difference in terms of intra-and postoperative outcome between PR and RR. Minor resections were more frequently performed: 169 in the PR group and 37 in the RR group. The 1,3 and 5 years OS has been $92 \%, 62 \%$ and $53 \%$ in the PR and $86 \%, 51 \%$ and $34 \%$ in RR group (P not significant). The recurrence rate at 1,3 and 5 years was respectively $40.5 \%, 56 \%$ and $61.2 \%$ in PR group and $44.6 \%, 60.5 \%$ and $64 \%$ in the RR.

Discussion: In patients with recurrent liver disease, repeated resection is possible to obtain survival rates comparable to those after primary hepatectomy with no additional cost in terms of short-term outcome.

\section{9}

\section{Impact of Metastatic Lymph Nodes Ratio in Gastric Cancer Staging After Limited Lymphadenectomy}

G. David, S. Rausei, F. Frattini, A. Leotta, V. Dadomo,

L. Ruspi, F. Rovera, L. Boni, G. Dionigi, R. Dionigi

Department of Surgery - University of Insubria - Varese

Introduction: To evaluate the prognostic value and staging accuracy of the metastatic lymph nodes ratio (NR) in gastric cancer patients underwent limited lymphadenectomy.

Methods: We classified 239 patients by 2 staging systems: UICC/AJCC TNM and NR-based; NR categories (NR0:positive/ negative nodes ratio $=0 \%, \mathrm{NR} 1: \leq 15 \%$; NR2:>15\%) were deter- mined by the best cut-off approach at log-rank test. The prognostic value of the 2 classifications was compared according to patients survival. The survival analysis was performed using the Kaplan-Meier method and the log-rank test and the Cox proportional hazards model.

Results: A significant difference in 5-year survival was observed in patients with NR1 versus NR2 ( $\mathrm{p}=0.003)$ : this statistical difference was stronger than statistical difference among 5-year survival rates of $\mathrm{pN}$-categories $(\mathrm{p}=0.019)$. At multivariate analysis only NR along with $\mathrm{pT}$ were found to be independent prognostic factors.

Conclusion: This study suggests that NR is a simply reproducible and highly reliable staging parameter with a strong ability to predict patients prognosis. NR-based classification is effective for staging of patients underwent limited lymphadenectomy also and could reduce the rate of "stage migration" compared with the 6th UICC/AJCC TNM staging system.

\section{0}

\section{Factors That Influence 12 or More Harvested Lymph Nodes in Resective RO Colorectal Cancer}

\section{E. Rimini, S. Scabini, E. Romairone, R. Scordamaglia, D. Pertile, V. Ferrando}

U.O. Chirurgia Oncologica e dei Sistemi Impiantabili, Direttore: Prof. Valter Ferrando, A.O.U. S. Martino Genova - Italia

Background: The number of lymph nodes required for accurate staging is a critical component in colorectal cancer (CRC). Current guidelines demand at least 12 lymph nodes to be retrieved. This study was designed to determine the factors that influence the number of harvested lymph nodes $(\geq 12)$ in resective R0 Colorectal Cancer early-stage CRC in a single institution.

Methods: Between july 2005 and decembre 2008, data on patients who underwent surgery for CRC were analyzed retrospectively (225). Data for a total of 139 R0-surgery patients were collected and all the tumor-bearing specimens were fixed with node identification performed. Several possible factors that influence 12 or more harvested lymph nodes were investigated and classified into four aspects: (1) operating surgeon, (2) examining pathologist, (3) patient, and (4) disease (tumor localization, tumor cell differentiation, tumor stage, type of resection).

Results: A total of 100 patients $(71.9 \%)$ with 12 or more harvested lymph nodes and 39 patients $(28.1 \%)$ with $<12$ lymph nodes were analyzed. The results demonstrate that within a single institution, tumor localization, depth of tumor invasion according to Dukes stage and grading were independent influencing factors of 12 or more harvested lymph nodes. Neither the operating surgeon nor the examining pathologist had significant influence on the number of harvested lymph nodes.

Conclusions: The number of harvested lymph nodes was highly variable in patients who underwent resection of R0 CRC. Neither the operating surgeon nor the examining pathologist had significant influence on the number of harvested lymph nodes. 
Therefore disease itself is the most important factor influencing the number of harvested lymph nodes.

\section{1}

\section{Mesenteric Fibromatosis, Follicular Thyroid Carcinoma in Struma Ovarifi and Reccurent Liposarcoma: Surgical Therapy for These Unusual Neoplasms}

\section{Risio, F. Selvaggi, P. Raimondi, S. Sulpizio, V. D'Alessandro, R. Cotellese, L. Napolitano, P. Innocenti \\ Dipartimento di Scienze Chirurgiche, Università degli Studi "G. d'Annunzio", Chieti-Pescara, Via dei Vestini, 66013, Chieti, Italia.}

Introduction: Mesenteric Fibromatosis (MF), Recurrent Liposarcoma (RL) and Follicular Thyroid Carcinoma in Struma Ovarii (FTCSO) are rare forms of human tumors. MS is often associated with other clinical syndromes and abdominal trauma. RL accounts for approximately 10-15\% of soft tissue tumors. FTCSO represents a unusual monodermal form of ovarian teratoma composed by thyroid tissue.

Materials and Methods: A retrospective analysis concerning the rare intrabdominal tumors was proposed. Morbidity, overall survival and disease free-survival together with pathological features were evaluated.

Results: The MS patient was admitted for recurrent epigastric abdominal pain. The CT scan demonstrated the presence of a neoplasm in the mesenteric region. The patient underwent to surgery without a well-defined diagnosis. A segment of jejunum together with the adjacent adipose tissue were excised. The definitive diagnosis was MF. In the case of RL, the diagnostic hypothesis was supported by MRI founding and the surgical treatment consisted of wild excision of the giant retroperitoneal mass. Microscopic analysis confirmed the hypothesis of RL. The patient affected by FTCSO was admitted for right abdominal pain and vomiting. Upper gastrointestinal endoscopy showed an ulcerative lesion of the stomach. In addition, CT scan demonstrated the presence of a large pelvic mass. A gastric resection sec. Billroth II together with bilateral hystero-annessectomy was performed. A diagnosis of FTCSO was made according to CD56 and Thyreoglobulin antigen positivity.

Conclusion: Our work highlights the need of more discriminated criteria for a correct preoperative diagnosis and appropriated multimodal therapeutic strategies in case of these rare diseases.

\section{2 \\ Case Report-Surgical Therapy of a Retroperitoneal Liposarcoma Weighing 15 Kg}

\author{
R. Romano, A. Rizzuto, G. Vescio, G. Sammarco, R. Sacco \\ Universita' Degli Study Magna Graecia Di Catanzaro
}

Introduction: Due to the late onset of symptoms, retroperitoneal liposarcoma are often diagnosed in advanced stages when adjacent organs have been infiltrated and the tumours have reached extensive sizes.

Background: Well-differentiated (WD) liposarcoma accounts for about $40 \%$ to $45 \%$ of all liposarcomas therefore representing the larger subgroup of adipocytic malignancies. It tends to occur equally in the retroperitoneum or the limbs followed by the paratesticular area and the mediastinum, with a peak incidence between the fifth and the seventh decades. WD liposarcoma is further subdivided in the adipocytic (lipoma-like), sclerosing, inflammatory, and spindle cell subtypes, of which the first two are by far the commoner. Surgery remains the first choice of therapy.

Case Report: A woman 49 years old underwent to resection for $15-\mathrm{kg}$ primary retroperitoneal well-differentiated (WD) liposarcoma that was removed en-bloc including spleen and pancreas with -tumour-free margins. Sex months later, the follow-up revealed a recurrence of the tumour with diaphragm and adrenal, which was surgically removed after chemoterapy. Since then, the patient has been without any signs of tumour recurrence or metastases.

Conclusions: Even extreme-ly large tumours can be removed safely. Local recurrence is common and occurs even after R0 resection. Recurrences should be surgically removed as this is the only treatment which has been shown to increase survival in even R1 and R2 situations.

\section{3}

\section{Prognostic Significance of R0-Resection in} Locally Advanced Gastric Cancer (LAGC)

\author{
F.C.M. Cananzi', A. Biondi ${ }^{2}$, R. Persiani ${ }^{1}$, M. Zoccali', \\ P. Mazzeo ${ }^{1}$, A. Tufo ${ }^{1}$, F. Ferrara', F. Sicoli', D. D'Ugo ${ }^{1}$ \\ ${ }^{1}$ General Surgery Unit, Catholic University, "A. Gemelli" \\ General Hospital, Rome, '2Emergency and General Surgery \\ Unit, Fondazione IRCCS "Ca' Grande" Policlinico \\ Maggiore, Milan
}

Introduction: Survival after R0-resection in LAGC remains poor suggesting that a true curative treatment has not been performed. Preoperative therapy has been proven to be effective in LAGC by large-scale randomized trials. Aim of this study is the evaluation of the effects induced by preoperative chemotherapy and its impact on survival.

Materials and Methods: 47 patients with LAGC underwent D2-gastrectomy after preoperative chemotherapy. The effects of preoperative treatment has been evaluated by a quan- 
titative analysis (percentage of residual vital tumor cells in the surgical specimens) and by a qualitative analysis (achievement of tumor-downstaging induced by any grade of pathologic response). T-down was assessed comparing laparoscopic and pathologic staging. $\mathrm{X}^{2}$ test was used to evaluate the statistical differences. Survival and prognostic significance were calculated by Kaplan-Meier method and log-rank test. Multivariate analysis was performed using Cox regression.

Results: Quantitative analysis of pathologic response did not show any prognostic significance. T-down was obtained in 25/47 patients. T-down was associated with a smaller mean tumor diameter $(34 \mathrm{~mm}$ versus $55 \mathrm{~mm}$ in no T-down;p=0.002) and a higher $\mathrm{R} 0$-resection rate ( $96 \%$ versus $72 \%$ in no T-down; $=0.04)$. 5-year overall survival was $55 \%$. In patients with a R0-resection (40/47) survival reached up to $63 \%$. At multivariate analysis, R0-resection was found to be an independent prognostic factor (R1-2/R0:HR $6.250 / 1, \mathrm{p}=0.002$ ).

Conclusion: In this study, R0-resection was the most important prognostic factor for LAGC treated by preoperative therapy. Patients with T-down had a definitely better chance of cure towards the achievement of a true R0-resection.

\section{4 \\ Prognostic Evaluation of Lymph Node Staging, Count and Dissection in Gastric Cancer}

\author{
F.C.M. Cananzi ${ }^{1}$, A. Biondi ${ }^{2}$, R. Persiani ${ }^{1}$, M. Zoccali ${ }^{1}$, \\ P. Mazzeo ${ }^{1}$, A. Tufo ${ }^{1}$, F. Ferrara ${ }^{1}$, F. Sicoli ${ }^{1}$, N. Zingaretti ${ }^{1}$, \\ D. D'Ugo' \\ ${ }^{1}$ General Surgery Unit, Catholic University, "A. Gemelli" \\ General Hospital, Rome, 'Emergency and General Surgery \\ Unit, Fondazione IRCCS "Ca' Grande" Policlinico \\ Maggiore, Milan
}

Background: According to the TNM classification, more than 15 lymph nodes are required for appropriate staging of gastric cancer. The aim of this study was to evaluate whether this number of resected lymph nodes affects survival as well.

Methods: A retrospective study was performed in 215 patients with gastric cancer, who had undergone R0-resection and completed a 5-year follow-up. Patients were classified according to the number of resected lymph nodes $(>$ or $<15)$, the anatomical extent of lymph node dissection (D2 vs D1) and according to the VII edition of the UICC/AJCC TNM staging rules. Survival estimates were determined using the Kaplan-Meier method and survival differences were assessed by the Log-Rank test. Multivariate analysis was performed using the Cox proportional hazards model.

Results: At univariate and multivariate analysis, resection of more than 15 lymph nodes was associated with significantly better survival results $(\mathrm{p}=0,007 ; \mathrm{HR}: 0,368)$. Stage-stratified survival varied significantly for N0 ( $82 \%$ vs $53 \%$; $=0,001)$ and N1 $(85 \%$ vs $52 \% ; \mathrm{p}=0,04)$ categories but no differences were found between N2 and N3. D2 dissection was associated with > 15 lymph nodes removed more often than $\mathrm{D} 1$ dissection.
Conclusion: In gastric cancer with limited lymph node involvement, removal of more than 15 lymph nodes, accomplished through a D2 lymph node dissection, significantly affected survival.

\section{5 \\ Preoperative Risk Assessment for Onco- geriatric Patients}

C. Stabilini, R.A. Audisio, B.L. van Leuween, M. Frascio,

F. Mandolfino, A. Azzinnaro, S. Perotti, E. Gianetta

Dipartimento di Chirurgia dell'Università di Genova

Introduction: Aim of the study is to develop a screening tool that allows surgeons to identify preoperatively, oncogeriatric patients most at risk of postoperative adverse events.

Methods: From jan 2009 to apr 2010, 34 patients $>70$ y $(47,1$ $\% \mathrm{~F}$, mean 77,1 , sd 5,5 y) were preoperatively evaluated using GFI, VES 13, TUG and PACE scores. Patients underwent major abdominal surgery for cancer (22 colic resection, 5 gastric resection, 2 distal pancreatectomy, 5 various ). Preoperatively 26 patients lived alone, $7(20,6 \%)$ were affected by cognitive impairment at MMT. Eight (23\%) were dipendent at IADL, 7 (21\%) at ADL, 8 (23\%) vulnerable at VES 13 . Nine (26\%) patients were depressed at GDS, $6(18 \%)$ fatigued at BFI, $4(12 \%)$ had an altered PS. Fourteen patients were frail (score $>3$ ) after GFI test. Two patients had a timed up and go test $>20$ "

Results: Mean postop stay was 13 days (SD 9,6). Two patients died postoperatively (6\%). Overall morbidity was 61,7\% (major $26 \%$ ). Alterations in MMT (p.007), IADL (p.0086), GDS (p.0151), PS (p.0001), VES 13 (p.0086) were statistically related to mortality. A poor PS (p.05) and VES 13(p.05) were significantly predictive of any postoperative morbidity, PS was also related to major morbidity (p.0192)

Conclusions: The use of a preoperative score is a valuable tool in the decision process concerning the candidacy of elderly cancer patients for surgery.

\section{6 \\ Electrochemotherapy in Head and Neck Cancer}

\section{J. Tassinari, G. Orlandino, T. Fabrizio}

Unit of Plastic Reconstructive Surgery, Scientific Oncology Institute, I.R.C.C.S. - C.R.O.B

Introduction: The scope of our study was the obtainement, with the electroporation, of the considerable reduction of the volume of the cancer or the creation of a plan in order to surgically procede.

It was used in patient affected by cutaneous or subcutaneous recurrences or metastases of different histological cancers, allready treated by standard therapeutic procedures (surgery, che- 
motherapy, radiotherapy) and at the moment otherwise judged non operable.

A modest dose of chemotherapy, specifically intravenous injection of bleomycin in bolus in 1 minute, is sufficient to reduce the tumor's volume.

Material \& Method: We had treated ten patients with this new procedure affected by head and neck cancer. Nine patients were affected by squamous cell carcinoma and one by metastases of squamous cell carcinoma. The age range was 65 to 86 , the average was 75,7. In all patients we found a reduced dimension of the tumour and bleeding stop.

Results: In all patients we releaved after twenty days a reduction of the tumour with objective and documented clinical and radiological examinations.

Conclusion: The results could be considered really effective in order to emphasize the possibilities of palliation treatment of the electrochemotherapy for the surgical approach towards cancer, considered otherwise inoperable for dimension and localization.

\section{7}

\section{Prognostic Factors of Non Sentinel Lymph Node Involvement in Melanoma Patients}

\author{
M. Urbani, P. Makovac, M. Roseano
}

Istituto di Clinica Chirurgica Generale e Terapia Chirurgica, Università degli Studi di Trieste, Trieste

\section{2nd Classified - SPIGC Award Session, Surgical Oncology}

Introduction: The purpose of our study is to identify the prognostic factors of non sentinel lymph node (NSLN) involvement in melanoma patients who underwent completion lymph node dissection (CLND) after positive sentinel lymph node (SLN) biopsy in order to establish those patients who may most benefit from a CLND.

Materials and Methods: We performed a retrospective analysis on 139 melanoma patients with positive SLN biopsy, 72 men (age 17-88) and 67 women (age 24-89), which came to our institute from January 2000 to August 2009. We considered patients' sex, primary melanoma location, histologic type, Breslow thickness, mitotic index, lymphocytic invasion and the size of SLN metastasis.

Results: We performed the CLND in 33 (in total 72 positive SLNs) of the 139 melanoma patients. At pathological examination only 8 patients $(24,3 \% \%)$ had NSLN metastasis. According to our data the univariate analysis (Fisher test) show that sex, primary melanoma location (trunk, extremity, other), histologic type (superficial spreading melanoma, nodular melanoma, other), Breslow thickness $(\leq 3 \mathrm{~mm}$ or $>3 \mathrm{~mm})$, mitotic index (low, moderate, high, unknown) lymphocytic invasion (absent, nonbrisk, brisk, unknown) and the size of SLN metastasis (macrometastasis or micrometastasis) are not significantly correlated with the risk of NSLN metastasis ( $p>0,05$ for all).

Conclusion: Although high mitotic index and ulceration of the melanoma provide an enhanced risk of NSLN positivity none of the factors considered in our study showed a statistically significant value of residual nodal disease.

\section{8}

\section{Treatment of Pseudomixoma Peritonei (PMP) by Cytoreduction Plus Hyperthermic Intraperitoneal Chemotherapy (HIPEC)}

\author{
M. Vaira', T. Cioppa ${ }^{1}$, C. Bing ${ }^{1}$, S. D'amico', G. Fiorentini², \\ M. De Simone \\ ${ }^{1}$ Departments of General and Oncological Surgery, and \\ ${ }^{2}$ Medical Oncology, San Giuseppe Hospital - USL 11, \\ 50053 Empoli, Florence, Italy
}

Background: Pseudomyxoma peritonei (PMP) is rare peritoneal carcinomatosis from appendiceal tumors with slowly progressive disease process and large amount of mucus containing rare epithelial cells. Encouraging results in the treatment of PMP have been reported by surgical cytoreduction of the primitive cancer, peritonectomy and hyperthermic intraperitoneal chemotherapy (HIPEC).

Patients and Methods: Til December 2009, 484 operations for PC were performed, 263 Cytoreduction+HIPEC, 67 consecutive patients affected by PMP were treated by cytoreductive surgery and the semi-closed HIPEC technique.

Results: The postoperative morbidity rate was $45 \%$; surgical morbidity was observed in 19 patients and medical complications in 9 cases. No postoperative deaths were observed. The survival data, 60 patients were anayized (the last 7 patients were considered only for complications rate, postoperative mortality and cancer features, not for OS or PFS because they were too recent). On 60 patients, five and ten years OS were $94 \%$ and $84.6 \%$; DFS was $80 \%$ and $70 \%$ years respectively. Survival probability may be good in patients with hystological type appendicular adenoma optimally cytoreduced (CCR-0). If preoperative chemotherapy was performed represented a negative prognostic factor with statistically significant impact both on OS and DFS.

Conclusion: Cytoreductive surgery plus HIPEC is associated with an acceptable risk of postoperative complications and mortality. This combined treatment results in DFS and OS rates that are not described for surgery associated with systemic chemotherapy and, in our opinion, may be considered the gold standard treatment for this rare tumor. 
179

\section{Treatment of Peritoneal Carcinomatosis from Colonic Cancer by Cytoreduction Plus Hyperthermic Intraperitoneal Chemotherapy (HIPEC)}

\author{
M. Vaira ${ }^{1}$, T. Cioppa ${ }^{1}$, C. Bing ${ }^{1}$, S. D'amico ${ }^{1}$, G. Fiorentini², \\ M. De Simone \\ ${ }^{1}$ Departments of General and Oncological Surgery, and \\ ${ }^{2}$ Medical Oncology, San Giuseppe Hospital - USL 11, \\ 50053 Empoli, Florence, Italy
}

Background: Peritoneal carcinomatosis (PC) is one of the routes of dissemination of abdominal neoplasms and is generally considered a lethal disease, with a poor prognosis by conventional chemotherapeutic treatments.

Some centers have reported encouraging results with cytoreduction and hyperthermic intraperitoneal chemotherapy (HIPEC). Many studies have reported encouraging results on overall survival (OS) and the diseasefree interval in patients affected by PC.

Patients and Methods: Til December 2009, 484 operations for PC were performed; in 263 cases cytoreduction plus HIPEC was carried out. Out of 80 operations for colonic cancer: 51 cytoreductions plus HIPEC, 12 cytoreductions+EPIC (early postoperative intraperitoneal chemotherapy) and 17 debulking or explorative laparoscopies/laparotomies were performed. The 51 patients who had undergone cytoreduction plus HIPEC for PC of colorectal cancer (CRC) were considered.

Results: The complication rate was $43 \%$ (22/51) and mortality rate $1.9 \%(1 / 51)$. The specific features of two groups were considered for the survival curves and complication rates with special reference to the peritoneal carcinomatosis index (PCI; range 0-39) and completeness of cytoreduction score (CCR:range 0-3). The mean survival time was 36.4 months but in Group A (patients operated from 1998 until at 2002), the mean survival time was 15 months compared to 32.5 months for Group B (patients operated from 2003 until at 2010) $(\mathrm{p}<0.001)$. The poor survival of Group A seemed to be related to higher PCI and CCR scores.

Conclusion: Correct patient selection based on a maximum PCI of 16, associated with complete cytoreduction (CCR-0), produced encouraging results in our experience.
180

Treatment of Peritoneal Carcinomatosis from Ovarian Cancer by Cytoreduction Plus Hyperthermic Intraperitoneal Chemotherapy (HIPEC)

M. Vaira1 , T. Cioppa1 , C. Bing ${ }^{1}$, S. D'amico1', G. Fiorentini², M. De Simone

${ }^{1}$ Departments of General and Oncological Surgery, and ${ }^{2}$ Medical Oncology, San Giuseppe Hospital - USL 11, 50053 Empoli, Florence, Italy

Background: Ovarian cancer may be considered as an"intraperitoneal disease" by itself. When surgical removal associated with systemic chemotherapy fails, usually, the history of the patients in characterized by poor prognosis. Some encouraging results have been reported by the treatment of PC from ovarian cancer by complete surgical cytoreduction, peritonectomy and hyperthermic intraperitoneal chemotherapy (HIPEC).

Materials and Methods: Til December 2009 we performed 484 operations for PC; in 263 cases cytoreduction plus HIPEC was carried out. 79 patients treated by cytoreduction+HIPEC were affected from recurrent ovarian cancer (4 patients treated twice). Statistical analysis was performed on 70 patients (last 9 too recent for evaluation). Two trials were applied: A) first peritoneal relapse after surgery+systemic chemotherapy, 3 months later from last CT administration (B) multiple relapse patients.

Results: On 70 patients, morbidity rate was $47 \%$ and mortalità rate was 7.14\%. Overall median survival rate was 46.4 mos, but in Group A was 58 mos and in Group B was 26 mos $(\mathrm{p}<0.06)$. Statistical analysis revealed that completeness of Cytoreduction (CCR-score) was related to survival: overall survival rate was 46.3 mos for CCR-0, 31 mos for CCR-1 $(\mathrm{p}<0.002)$. Survival rate in group A: CCR-0: 48 mos, CCR-1: 25.6 mos ( $<<0.942)$; in Group B: CCR-0: 31 mos, CCR-1: 11.7 mos $(\mathrm{p}<0.012)$

Conclusions: Cytoreduction plus HIPEC seems to have an impact on prognosis in selected patients affected from peritoneal carcinomatosis from ovarian cancer. Better selection of patients and second-look surgery in high risk-patients have to be investigated to improve those encouraging results. 


\section{1}

\section{Evaluation of Extensive Cytoreductive Surgery and Hyperthermic Intraperitoneal Chemotherapy in Patients with Peritoneal Relapse of Epithelial Ovarian Cancer}

\author{
C. Vallicelli', L. Ansaloni' F. Catena ${ }^{1}$, D. Cavaliere ${ }^{2}$ \\ M. Framarini' F. Gazzotti', F. Tauceri' ${ }^{2}$, F. Coccolini ${ }^{1}$, \\ D. Di Pietrantonio², P. De laco ${ }^{1}$, C. Ghermandi', \\ V. Agnoletti ${ }^{2}$, G.M. Verdecchia ${ }^{2}$, A.D. Pinna ${ }^{1}$ \\ ${ }^{1}$ U.O. Chirurgia Generale e dei Trapianti, Policlinico S. \\ Orsola-Malpighi, Bologna, ${ }^{2}$ U.O. Chirurgia e Terapie \\ Oncologiche Avanzate, Ospedale G.B.Morgagni-L. \\ Pierantoni, Forlì
}

\section{1st Classified - SPIGC Award Session, Surgical Oncology}

Background: Aggressive cytoreductive surgery (CS) has been shown to have a positive impact on survival of patients with ovarian cancer. After first-line chemotherapy, $47 \%$ of patients relapse within 5 years, and median survival after second line chemotherapy is $10-15$ months. Adding hyperthermic intraperitoneal chemotherapy (HIPEC) to surgical cytoreduction could further control peritoneal surface spread of disease.

Aims: The aim of this study was to determine morbidity and mortality, regional relapse-free survival and, preliminarily overall survival after combining CS with HIPEC for the treatment of peritoneal carcinomatosis from ovarian epithelial cancer relapsed after prior chemotherapy.

Patients and Methods: Thirty women affected with such a relapse were included. Patients underwent extensive cytoreductive surgery including radical tumor resections and peritonectomy, followed by intraoperative HIPEC with cisplatin.

Results: Complete surgical cytoreduction down to nodules less than $2.5 \mathrm{~mm}$ (CC0-CC1) was obtained in 23 patients $(77 \%)$. One patient died postoperatively from a pulmonary embolism. Major postoperative morbidity was $5 / 30$ (16.7\%). We registered one case of intestinal anastomotic leakage, a spontaneous ileum perforation, a postoperative cholecystitis, a hydrothorax, and one patient with bone marrow toxicity. Kaplan-Meier estimates of median locoregional relapse-free survival and median overall survival were 17.1 months and 28.1 months, respectively. Patients with $\mathrm{CC} 0-\mathrm{CC} 1$ had locoregional relapse-free and overall survival rates of 24.4 and 37.8 months, whereas the remainder had survival rates of 4.1 and 11.0 months.

Conclusions: CS combined with HIPEC is feasible with acceptable morbidity and mortality and seems to promise good results in selected patients affected by peritoneal carcinomatosis from ovarian cancer.

\section{2 \\ Diagnostic Lymphoscintigraphy to Plan Anaesthesia for Sentinel Node Biopsy Melanoma Patients}

\author{
G.c. Vitali, M. Zonta, F. Verrecchia, J. Soteldo, G. Spadola, \\ G. Trifirò, A. Testori \\ European Institute of Oncology ( IEO ), Milan, Italy
}

Introduction: Sentinel Node Biopsy (SNB) is standard for certain patients with melanoma and can be performed under local or general anaesthesia ${ }^{1}$. Lymphoscintigraphy (LS) maps the lymphatics draining cutaneous melanoma and is reproducible ${ }^{2}$. This study aims to determine the role of diagnostic LS for planning SNB anaesthesia and to further evaluate the reproducibility of LS.

Methods: 109 select patients with primary cutaneous melanoma who underwent two preoperative LS between 2001 and 2006 were retrospectively studied. We performed SNB under general anaesthesia if the LS revealed hotspots in the neck or in multiple basins. At IEO, day surgery for local anaesthetic procedures means rescheduling patients if general anaesthesia is required. We studied 74 patients with truncal melanoma (group 1), 32 patients with melanoma below the knee (group 2) and 3 patients with melanoma of the distal upper limb (group 3). A diagnostic LS was initially performed followed by a LS immediately preoperatively.

Results: The lymphatic drainage of group 1 patients following diagnostic LS was to a single basin in $45.2 \%$ and to multiple basins in $54.8 \%$. In group 2 patients, drainage was to a single basin in $81.8 \%$ and to multiple basins in $18.2 \%$. In group 3, drainage was to a single basin in two patients. Both LS were identical in $87.2 \%$ patients with differences only in groups 1 and 2 .

Conclusion: Lymphatic drainage of truncal and distal lower limb melanoma to single or multiple basins is unpredictable and diagnostic LS is recommended before planning SNB anaesthesia in these patients. LS was reproducible when repeated prior to SNB.

183

\section{Electrochemotherapy with Bleomycin: A Local Treatment with Possible Systemic Implications}

\author{
G. C. Vitali, F. Verrecchia, J. Soteldo, A. Di Pietro, \\ G. Spadola, C. Martinoli, S. Gandini, P.F. Ferrucci, A. Testori \\ European Institute of Oncology ( IEO ), Milan, Italy
}

Introduction: A new, promising application of bleomycin was discovered and developed by Mir in 1991, combining the drug with electroporation. This new technique was called electrochemotherapy (ECT): cell-membrane permeabilization by electric pulses delivered locally at the site of superficial tumor localizations allows an increased bleomycin concentration in tumor cells. Since 1991 more than 300 articles were published on the issue and experiences from many European institutes seem to be very promising. At present ECT is used to treat different cutaneous and subcutaneous tumors. Favorable results in treating superficial metastatic 
lesions have been published, with a $70 \%$ to $90 \%$ complete response rate for each treated nodule.

Methods: In our Institute from 2006 to 2009 we treated 75 patients with superficial cancer lesions; 58 patients had metastatic melanomas and 15 patients had squamous or large basal cell carcinomas.

Results: We observed an overall response (OR)rate of $90 \%$; a complete response (CR) rate of $72 \%$ in melanoma patients and a CR rate of $65 \%$ in non melanoma patients. Of the 75 patients, only 8 relapsed in the treatment field. The procedure, either under general or local anesthesia, was well tolerated with minimal side effects or discomfort for the patients.

Conclusions: We conclude that ECT is a safe and well tolerated procedure with quality of life improvement especially in a palliative setting.

Furthermore, we are currently evaluating the development of immune responses elicited by ECT, comparing serum levels of pro-inflammatory cytokines as well as frequency and activation of $\mathrm{T}$ cell populations in peripheral blood of selected patients, before and after therapy.

\section{4}

\section{Presentation of Multicentric Trial About Sentinel Node Identification with Sentinella ${ }^{\circledR}$ Intraoperative Gamma Camera vs Traditional Gamma Probe}

\author{
G.C. Vitali, F. Verrecchia, J. Soteldo, G. Spadola, \\ P.F. Ferrucci, A. Di Pietro, F. Cataldo, A. Testori \\ European Institute of Oncology ( IEO ), Milan, Italy
}

Introduction: By its natural history, melanoma starts as a locoregional disease characterized by a progressive worsening of the prognosis as the disease, from the primary lesion, diffuses to the nodes and then to the rest of the body. Actually, when the disease has involved locoregional nodes, it does not always spread systemically. Therefore, sentinel lymph node biopsy (SNB) for melanoma patients acquires a strong prognostic significance.

This is a minimally invasive procedure designed to identify and remove specific lymph nodes that may contain metastatic cancer cells. Clinical trials have demonstrated that a negative SN is highly predictive for the absence of metastatic disease in melanoma patients.

The $\mathrm{SN}$ is traced by a radioactive colloid, using a hand-held gamma probe intraoperatively to identify lymph nodes likely to contain metastases from the primary lesion.

Methods: Sentinella ${ }^{\star}$ is a surgical device composed of a portable gamma camera with laser pointing system, a traditional hand-held gamma probe, a Gadolinium pointer with a touchscreen monitor and software to process images and to write medical reports.

Over the last year, in our institution we performed 68 SNB using Sentinella ${ }^{\oplus}$ with $100 \%$ node identification and excision rate.

A trial is currently ongoing, to evaluate whether the use of Sentinella ${ }^{\circledR}$ device will improve accuracy in locating sentinel nodes. At first, nodes are detected with traditional gamma probe; after their removal, Sentinella gamma camera is used to check for any other sentinel nodes.

Conclusion: The primary endpoint of this trial is to evaluate whether the number of sentinel nodes removed with Sentinella equipment is higher than using traditional gamma probe alone. We also want to evaluate the number of positive sentinel nodes identified using Sentinella intraoperative equipment vs using only a traditional intraoperative probe. A third endpoint is to verify the false negative SN rate. Furthermore, cost-benefit analysis of this approach will be considered.

\section{Plastic Surgery}

\section{5 \\ Complex Lower Limb Injuries: Reconstructive Strategies Selection

\author{
E. Pataia, M. lera, R. Maffia, V. Ceccolini, V. Langella, \\ C. Alfano \\ Department of Plastic Surgery, University of Perugia Chief \\ Professor Carmine Alfano
}

Introduction: Traumatic lower limb injuries repair has always been a challenge for plastic surgeons.

Both local anatomy and functional needs require the best quality soft tissue reconstruction in the less time. Complex open fractures with wide bone exposition and weight bearing spots necessitate good quality tissue coverage. The vascular impairment usually exceeds the injured area.

Materials and Methods: At the Plastic Surgery Department of the Perugia University we treated 32 complex lower limb traumas from September 2006 through March 2010. Fascio-cutaneous pedicled flaps were used in 17 cases, muscular pedicled flaps in 9 cases and muscular free flaps in 6 cases.

Results: We obtained a good repair with high quality tissue, in a single surgical step, in all of the treated patients. We observed postoperative venous stasis in 2 out of 17 fascio-cutaneous distal pedicled flaps and marginal suture failure in 1 heel coverage. Both the complications were successfully treated with advanced wound dressing and leeches.

Conclusions: Quick recovery in lower limb injuries allows early mobilizations which is essential in order to obtain a good functional result.

Pedicled flaps offer an easy and reliable solution in most cases.

Use of microvascular free flaps is limited by the extension of vascular impairment around the lesion. However free flaps are actually the best option in large bone exposure. 


\section{6}

\section{Use of Biotechnologies in Cutaneous Injuries Limb Repair}

\author{
E. Pataia, S. Arleo, F. Somma, G. Menichini, A. D'antonio, \\ C. Alfano \\ Department of Plastic Surgery, University of Perugia, \\ Chief: Professor Carmine Alfano
}

Introduction: "Reparative Surgery" is actually evolving toward "Regenerative Surgery". Reparative surgical techniques necessarily result in scar tissue, whereas regenerative procedures let us obtain repair with healthy tissue. Cutaneous limb injuries require quick good quality repair, in order to obtain good functional results.

Materials and Methods: At the Plastic Surgery Department of the Perugia University we treated 63 cutaneous limb injuries from September 2006 through March 2010: 30 pressure ulcers, 23 traumas, 6 burns, 4 vascular ulcers.

We used Vacuum Assisted Therapy, Versajet, Advanced Dressing, Dermal Substitutes, Autologous Cellular Cultures, Platelet Gel and Lipostructure.

In 23 patients regenerative procedures were employed together with surgery (Skin grafts, skin flaps).

Results: Comparison of so many different techniques and patients is difficult; nevertheless we observed good and quick results in all the treated cases.

Conclusions: Both scientific research and commercial aims are running together to obtain better and better solutions for skin repair. We are now able to choose among so many different techniques the most appropriate procedure for each lesion and patient. Adult adipose stem cells and growing factors actually appear to be the best hope for autologous tissue regeneration.

Although these new technologies are quite expensive, reduction of number of surgical procedures and shortening of the recovery time make them cost effective.

\section{7}

\section{Multiple Vascular Malformations: Case Report}

\section{S. Chiummariello, M. lera, R. Maffia, S. Arleo, C. Calzoni, C. Alfano}

Department of Plastic Surgery, University of Perugia, Italy. Chief: Prof. C. Alfano

Introduction: Association of multiple vascular malformations on the face is a rare condition; a rarity is also an arteriovenous malformation (AVM) that uses a venous malformation as the draining vein.

We report a case of a mixed vascular malformations in the same patient which was treated by multidisciplinary approach.

Materials and Methods: At 14 year-old Caucasian boy was referred to our department with a long history of a painless lower lip for an extensive vascular malformation involving lower lip, oral cavity and cheek.

The patient also reported functional alteration for eating, speaking and air inflation, sleeping and cosmetic disturbance, serious psychological distress.

The angiography revealed an arterio-venous malformation (AVM) of the lower lip associated with a venous lesion of the other districts.

Facial magnetic resonance imaging (MRI) showed that the mass also involving pterygo-maxillary fossa and horizontalis branch of the mandible.

A therapeutic choice was a combination of embolization and surgery; we postponed a surgical demolition of the jaw and the tongue.

Results: The final pathologic examination confirmed double vascular malformations.

Ipertrophic scars were a post-operative complications.

To date (20 months follow-up) clinical examination shows a normal mouth anatomy; we have excellent patient satisfaction as well as functional good results.

Conclusion: We think that the AVM uses the venous malformation as its draining vein.

The patient has no evidence of local recurrence; venous malformation of the mandible and the tongue is stable.

An optimal management of patient with vascular malformations includes a multidisciplinary approach with critical consideration of all potential treatment procedures.

\section{8 \\ Skin Sparing Mastectomy: Morphological Results and Quality of Life in Breast Cancer Patients}

\author{
A.F. Armenti, M. Mazzocchi, L.A. Dessy, E. Trignano, \\ N. Scuderi
}

Introduction: Skin-sparing mastectomy (SSM) allows mastectomy to be carried out preserving the natural cutaneous shell of the breast. It's oncologically safe for all patients affected by stage I and II breast cancer, multicentric tumours, tumors larger than $4 \mathrm{~cm}$ in size, DCIS, which are all eligible for prophylactic mastectomy. This approach no longer requires subsequent radiation treatment, enables immediate breast reconstruction (IBR),ensuring an excellent aesthetic outcome. In our study, we analize patients undergone SSM with immediate breast reconstruction in terms of morphological outcomes and quality of life (Qol).

Materials and Methods: From January 2000 to December 2004, 56 patients were treated with SSM with IBR. Their degree of satisfaction, was evaluated with the SF-36 questionnaire. We evaluated the anterior projection, the degree of mammary ptosis, the alignment of the inframammary folds, the contour, the symmetry, the mammary volume and the form and the colour of CAC.

Results:Among 56 patients, 8 (14\%) had reconstruction with pedicled TRAM flap, 12 (21\%) with latissimus dorsi flap and implant, 36 (64\%) only with implant. At the five years follow up, 
the frontal projection was identical in $70 \%$ of the patients, mammary ptosis was identical in $65 \%$. The alignment of the inframammary folds was maintained in $73 \%$. In physical health component the total score was 58.5 and in $\mathrm{t}$

\section{9}

\section{Advances in Imaging for Perforator Flaps Breast Reconstrucion}

\author{
M. Atzeni', A. Corona 1, L. Saba², W. Rozen ${ }^{3}$, D. Ribuffo \\ ${ }^{1}$ Department of Surgery, Section of Plastic Surgery, \\ ${ }^{2}$ Department of Radiology, Cagliari University Hospital, \\ 3Jack Brockhoff Unit Research, Melbourne University, \\ Australia
}

Background: Breast reconstruction performed with the use of perforator flaps, especially from the abdominal wall donor site, represents an important option, although with potential complications including donor site morbidity and impaired flap viability. In order to decrease this percentage of complication, an accurate preoperative evaluation of the vascular anatomy of the abdominal wall, by the means of instrumental devices, from EcoColorDoppler to AngioCT, is highly desiderable in improving the surgical strategy in abdominal flaps for breast reconstruction.

Materials and Methods: In the last five years we have focused the attention on the potential benefits, including reduced intramuscular dissection, faster and safer dissection, reduced donor site morbidity and improved flap vascularity and survival, by the use of AngioCT, compared to ECD, in abdominal perforator flaps planning, due to the possibility to value exactly position, caliber, course of perforator vessels branching from Deep Inferior Epigastric Artery, to evaluate the variability of the Superificial Inferior Epigastric Artery or localization, course and anatomical variations of Deep Superior Epigastric Artery and apply these studies to perform DIEP and pTRAM flaps.

Results: The use of CTA was associated with decreased operating time, decreased complications related to flap viability and a statistically significant reduction in donor site morbidity.

Discussion: Accurate identification of the main perforators achieved with a very satisfactory concordance between AngioCT and surgical findings, compared to ECD, permit us to choose in preoperative time the side with the best perforator to planning breast reconstruction with abdominal perforator flaps promoting a significant reduction in operating time and complication rate.

\section{0}

\section{The Role of Carbon Dioxide Therapy in the Treatment of Chronic Wounds}

M. Campana, C. Brandi, A. Brafa, M. Calabrò, A. Campa,

M. Vaccaro, L. Rosato, R. Perello, L. Ceccaccio, G. Nisi,

L. Grimaldi, C. D'Aniello

Plastic Surgery Unit, Department of General and Specialist Surgery, Siena University, S. Maria alle Scotte Universitary Hospital, 53100 Siena, Italy

Introduction: A wound is defined as chronic when it does not heal according to the normal repair times and mechanisms. This particular condition may be principally due to local hypoxia. Carbon dioxide $\left(\mathrm{CO}_{2}\right)$ therapy refers to the transcutaneous or subcutaneous administration of $\mathrm{CO}_{2}$ for therapeutical effects on both microcirculation and tissue oxygenation. In this study, we report the clinical and instrumental results of the application of $\mathrm{CO}_{2}$ in the therapy of chronic wounds.

Materials and Methods: The study included 70 patients affected by chronic ulcers. The patients were selected by aetiology and wound extension and equally divided into two homogeneous groups. In group $\mathrm{A}, \mathrm{CO}_{2}$ therapy was used in addition to the routine methods of treatment for such lesions. In group B, patients were treated using routine methods alone. Both groups underwent to instrumental (laser doppler flow, measurement of $\mathrm{TcPO}_{2}$ ), clinical and photographic evaluation.

Results: In the group that underwent subcutaneous treatment with $\mathrm{CO}_{2}$ therapy, the results highlighted a significant increase in tissue oxygenation values, which was confirmed by greater progress of the lesions in terms of both healing and reduction of the injured area.

Conclusion: Considering the safety, efficacy and reliability of this method, even if further studies are necessary, we believe that it is useful to include subcutaneous carbon dioxide therapy in the treatment of chronic wounds.

191

Treatment of Breast Ptosis Using a

"Self-Implant": A Personal Technique

M. Campana, G.Nisi, M. Calabrò, A. Campa, M. Vaccaro,

L. Rosato, P. Perello, L. Ceccaccio, A. Brafa, C. Brandi,

L. Grimaldi, C. D'Aniello

Plastic Surgery Unit, Department of General and Specialist Surgery, Siena University, S. Maria alle Scotte Universitary Hospital, 53100 Siena, Italy

Introduction: The best way to give back a natural shape to severe ptosic breasts without using implants remain the reshaping of the lower pole like a "self-implant".

During the last 30 years, many techniques are described for improving the mastopexy results.

In our opinion the best results could be obtained using self tissue.

Eur Surg Res 2010;45:158-307 
Materials and Methods: From January 2006 to june 2008, 25 patients underwent mastopexy and 20 patients underwent breast reconstruction with controlateral mastopexy simmetrization. In all this patients we have performed the mastopexy with associated to this kind of breast tissue reshuffle.

Results: The follow-up period was 18 months during which all patients were pleased with their results. We didn't encountered case of steatonecrosis or other early complication.

Conclusion: In our technique we obtain a reducing of the breast weight graving on the skin envelope and, in the same time, a natural shape with an improving of upper pole fullness, projection, thickness and an improving of inframmamary fold definition. The lateral side and lower pole of the breast can be centralized and used to filling the breast like an implant defining at the same time the breast countour and inframammary fold.

Placing the parenchimal steatch the operator can modulate the grade of plication and adapting it to the controlateral breast.

\section{2}

\section{The Use of Abdominal Flaps for Breast Reconstruction}

\author{
L. Canta, M. Moio, G. Molea \\ Cattedra di Chirurgia Plastica, Ricostruttiva ed Estetica, \\ Direttore: Prof. G. Molea \\ Università degli Studi di Napoli "Federico II"
}

Introducrion: The mammary reconstruction pursues the obtainment of a breast with shape, volume and consistency similar to the normal breast. It can be achieved by using the own patient's tissues and/or alloplastic implants. The most appropriate technique is choosed after the assessment of the general conditions, age, anatomy, remnant tissues and expectations.

Materials and Methods: The lower abdominal region offers a multitude of flaps for breast reconstruction. The TRAM flap procedure uses skin, fat and muscle from the lower abdominal wall. It can be pedicle, leaving the flap attached to its original blood supply (perforators of the Superior Epigastric vessels) and tunnelling it under the skin to the breast area, or free, requiring the use of microsurgery to connect the vascular supply to the internal mammary or toracodorsal vessels. The DIEP flap only requires the removal of skin and fat. No muscle is sacrificed. The blood supply comes from the perforator arteries of the deep inferior epigastric vessels. The flap is connected to the patient's chest using microsurgery.

Results: The TRAM flap procedure allows to shape a soft and natural breast with results that mimic a "tummy tuck" procedure.

The DIEP flap has significantly fewer abdominal complications and side-effects (pain, bulging, hernia, strength loss) because the majority of the abdominal muscle is spared.

Conclusions: Breast reconstruction is fundamental for the acceptance of mastectomy, improving the patient's self image and confidence. The abdominal area is an ideal donor site for mammary reconstruction. An adequate planning and patient selection can achieve the best results choosing the most adequate technique.
193

\section{The Broken of Implant Breast : Study by Imaging}

\author{
D.G. Colombo ${ }^{1}$, D.A. Garlaschi², D.M. Bormioli ${ }^{3}$ \\ ${ }^{1}$ Università degli Studi di Genova - Scuola di \\ specializzazione in Chirurgia Plastica e Ricostruttiva, \\ 2Dirigente Medico, U.O.C. di Senologia - Ospedale S. \\ Martino - Genova, ${ }^{3}$ Direttore U.O.C. Chirurgia Plastica e \\ Maxillo-facciale- Ospedale S.Corona - Pietra Ligure-Savona
}

In recent years, there has been an increasingly insistent recourse to plastic surgery in the case of breast implants.

On the one hand it is caused by the increasing number of breast cancer diagnosed, but on the other hand because it is increasing the need for patients to seek a physical form consistently better ( in the occidental world it is linked with the typical imagine of breast and both tonic).

For that reason, we assist at the increasing use of imaging techniques that are becoming really accurate and sophisticated in order to study adequately the breast prothesis and their integrity.

Imaging diagnostic pass through several options: mammografy and ecografy; however, actually, the gold standard to have an accurate analysis of patients with breast implants is represented by MRI.

The aim of our study was that, after a brief panoramic on the main radilogical techniques to visualize the implants, to focus on the main MRI signs, capable of studying contractures (clinically detectable) or breakage of the prosthesis. All this, to give at the surgeon an adequate tool in order to discern alteration of the breast implants.

For all that reasons, it has been necessary a co-operation between plastic surgeon and radioligist specialized to guarantee a proper management of patients and the complication which may occour.

\section{4 \\ Minimally Invasive Correction of Adult Pectus Excavatum}

\author{
D.G. Colombo ${ }^{1}$, D.V. Ruvolo ${ }^{1}$, D. B. Scarsi ${ }^{2}$, M. Bormioli ${ }^{3}$ \\ ${ }^{1}$ Università degli Studi di Genova - Scuola di \\ specializzazione in Chirurgia Plastica e Ricostruttiva, \\ 2Università degli Studi di Genova, ${ }^{3}$ Direttore U.O.C. \\ Chirurgia Plastica e Maxillo-facciale- Ospedale S.Corona - \\ Pietra Ligure-Savona \\ U.O.C. Chi. Plastica - Santa Corona Hospital - Pietra \\ Ligure Italy
}

This congenital deformity, that in Italy occurs in an estimated 1 in 8.000 births, may vary from moderate till severe condition.

Recently the Nuss procedure, normally used in Pediatric Surgery; has been introduced also in adult surgery: this had permitted to threat patients usually contrary to a classic, invasive surgery. 
Pre-surgery examination are ECG, EcoCardio, Rx thorax, CT; we used a $3 \mathrm{D}$ CT reconstruction to project the place, the number and the size of the barrs.

The surgery begins with toracoscopy along the right, between 7 th and 8 th space, with a $30^{\circ}$ opt.

Is then performed a tunnel through which will be slipped one or two steel bars, shaped to adapt to correct chest deformity. Generally the bar used for adult patient is shorter than the ones used for adolescents.

Furthermore, when treating adults it is mainly important to stop both ends of the bar with appropriate devices. Pre-surgery physiotherapy should assumes mainly importance, together with post surgery rehabilitation.

When th thoracic depression is moderate without fuctional copmpromission or the patient refuses the Nuss procedures, we use to repair the defect by a lipofilling in presence of a subcutaneous well represented or we used the new jaluronic acid : MACROLANE.

\section{5 \\ Nipple Reconstruction: A Prospective Cohort Study About Arrow Flap}

\section{Dessena, A. Balzano, A. Bulla, F. Marongiu, C. Rubino \\ Plastic Surgery Unit, University of Sassari}

Introduction: Nipple/areola reconstruction is the final phase of breast reconstruction.

Nipple projection is the critical parameter to evaluate a technique during follow-up.

In 2003 we described our variant, named "arrow flap".

The nipple, considered as a cylinder, can be unfolded into a circle attached to a rectangle. One side of rectangle will be marked as an arrow point, the other side as an arrow tail, a triangular area above the circle is added and they will be deepithelised.

We elevate the arrow flap on a sub-dermal plane, with just a little superficial subcutaneous fat. Then, the flap donor site is closed directly, arrow point and tail are fixed together, the cup is fixed to the wings and the flap base to the surrounding skin.

Materials and Methods: Our previous study showed a mean residual projection of $49 \%$ of the arrow flap.

After a 3 years follow-up we present a prospective cohort study about the arrow flap alone.

Forty women, after mammary reconstruction, underwent nipple reconstruction using our technique.

Nipple projection was then carefully measured in each patient during a 3-year period.

Results: The mean residual projection after 2 years is $46.55 \%$, after 3 years it is $46 \%$.

Conclusion: We can conclude that nipple reconstructed with our after two years is stable and maintain similar projection during the third year of follow-up.
196

\section{A New Sympathectomy Technique for the Treatment of Palmar and Axillary Hyperhydrosis}

D.M. Galati, E. Raposio

Università degli studi di Parma, Scuola di Specializzazione in Chirurgia Plastica Ricostruttiva ed Estetica, Direttore

Prof. E.Raposio

Introduction: Palmar and axillary hyperhydrosis could be idiopathic or caused by pathologies such as hyperthyroidism, pheochromocytoma and hypothalamic pituitary axis diseases. This problem is highly disabling and embarrassing for patients.

At present, the endoscopic thoracic sympathectomy technique is the treatment of choice for palmar and axillary hyperhydrosis. This method relies, at least, on double throcar insertion (on the side) and/or carbon dioxide insufflation. Although this approach, compared to traditional "open" sympathectomy techniques, carries with it the smallest number of post-operative complications, it still determines a certain amount of postoperative discomfort as well as a risk of complications related to carbon dioxide insufflation, as intraoperative profound bradycardia and hypotension due to mediastinal shift and postoperative subcutaneous emphysema.

Materials and Methods: From December 1995, over 500 patients with palmar and axillary hyperhydrosis have been included in the study and we have been using a minimally-invasive endoscopic transthoracic sympathectomy technique, performed by a single-entry specifically modified thoracoscope and without the need for the carbon dioxide insufflation, with the aim to reduce the drawbacks associated with the above mentioned, currently adopted endoscopic techniques.

Results: The effects of the sympathecotomy are immediate, as the patients wake up with warm and dry hands and axillae. The surgical procedure is said to have short operation time (60 minutes in average), short postoperative course (2days in average) with rapid return to their normal activities with out complication.

Conclusions: In our opinion, this "single-entry" technique, compared to the other reported approaches, should theorically minimize any damage to the intercostals neurovascular bundle, while allowing to avoid the complications related to carbon dioxide insufflation. 
197

\section{The Medial Pedicle for Reduction Mammaplasty : Experience Liègeoise with Hall-Findlay Technique}

\author{
M. Grieco ${ }^{1}$, D. Nardella ${ }^{2}$ \\ ${ }^{1}$ Department of Plastic and Reconstructive Surgery - \\ University of Foggia, "Ospedali Riuniti" Hospital, Foggia \\ (Italy), Prof. A. Portincasa, ${ }^{2}$ Department of Plastic and \\ Reconstructive Surgery - "CHU"de Liège, Sart Tilman - \\ LIEGE (Belgium)
}

Introduction: The aim of reduction mammaplasty is to recreate an aesthetic volume of the breast, preserving the normal functions. The superomedial pedicle was first described by Orlando in 1975; in 1999 the technique was modified by E.J. HallFindlay.

Materials and Methods: From January 2008 to March 2010, 23 patients have been operated: 5 patients between October 2009 and March 2010, in the Plastic Surgery department of CHU de Liège. The average age is 35 years. The Wise keyhole reduction pattern technique was used for all patients. The pedicle is drawn at the centre of the areola (wide $6-8 \mathrm{~cm}$, thick $2 \mathrm{~cm}$ ). Vertical branches are long 6-8 $\mathrm{cm}$ to prevent bottoming out. An inverted $T$ scar is applied to avoids the redundancy of skin.

Results: Mean follow-up was 9 months. The resection is done in block. The mean resection weight per breast was $520 \mathrm{~g}$ on the left and $560 \mathrm{~g}$ on the right. The sensibility of NAC is measured with Semmes-Weinstein monofilaments: $90 \%$ of the patient mentioned a normal sensibility after 6 months. Suction drainages was not applied. Any fixation of the pedicle was performed. The form, size and breast scars was accepted by $90 \%$ of the patients. No major complications was observed.

Conclusions: Medial pedicle removes the inferior tissue susceptible of the effect of gravity and leaves tissue desired for shaping. The use of the medial pedicle technique for breast reduction is safe, reliable and produces satisfactory aesthetic result.

\section{8 \\ The Reverse Island Forearm Flaps for the Dorsal Hand Reconstruction}

F. Idone, M. Riccio, P.P. Pangrazi, A. Bertani

Chirurgia Plastica

Introduction: Injuries of the dorsum of the hand, characterized by wide loss of soft tissue, are caused by many mechanisms, with prevalence of mechanical traumas and particularly crushing injuries. The authors present their experience with these injuries, underlining the indications how to choose the most ideal flap to reconstruct the dorsum of the hand.

Methods: Eleven patients which sustained severe injuries to the back of the hand were recruited and treated with rotation of island reverse flap from the forearm for the teatment of isolated soft tissue lesions or more complex injuries.
Results: The gold standard for the treatment of injuries on the dorsum of the hand is through the use of the distally based Radial Artery Flap which is reliable, easy to dissect, and capable of covering large exposed areas. Conclusions: The use of the radial forearm fascia flap is usually preferred to the fascio-cutaneous one, because the former leaves a smoller scar at the donor site and because it offer greater thinnes and versatility, which makes it ideal for reconstructing the soft tissues on the dorsum of the hand. On the other hand the radial fascio-cutaneous flap is indicated when the lòoss of soft tissue on the dorsum of the hand is associated with exposed, comminuted and contaminated fractures that require a more extensive coverage of the osteosynthesis.

\section{9}

\section{Experience with the Becker-35 Expandable Implants in Reconstructive Breast Surgery}

\author{
M. Iera, S. Chiummariello, E. Pataia, S. Arleo, R. Maffia, \\ V. Ceccolini, C. Alfano
}

Department of Plastic Surgery, University of Perugia, Italy. Chief: Prof. C. Alfano

Introduction: Amastia is a breast deformity that causes deep psycological problems in women and disturbes their social relations.

Breast reconstruction with tissutal expanders is a technique involving in the seventies.

Expanders must be replaced with definitive prosthesis when the expansion is completed. In the eighties Becker used a permanent tissue expander that becomes popular because it avoids expander-implant exchange and gives the patient some control over the final breast size.

Materials and Methods: From October 2006 to December 2009, in our Department, Becker permanent tissue expanders were inserted in 106 women; 81 were immediate reconstructions (65 monolateral and 16 bilateral) and 25 delayed breast reconstructions ( 20 monolateral and 5 bilateral).

The age varied from 45 to 68 years (average 56.5 years).

67 patients underwent to radical mastectomy sec. Madden; 19 women were treated with skin sparing mastectomy, 13 patients with nipple sparing mastectomy and 7 cases with skin reducing mastectomy.

Results: In the majority cases we achieved good aesthetic results. Mean follow-up was 20 months.

Complications included: capsular contracture (7\%), implant dislocation $(5 \%)$, valve dislocation $(4 \%)$, seroma or hematoma (4\%). One patient developed periprosthetic infection; in this case the prosthesis was removed.

Conclusion: Breast reconstruction, both immediate and delayed, with Becker permanent tissue expander is an excellent technique who can be used as an alternative to other techniques.

The combined expander implants permits a gradual augmentation of the breast in order to better volume simmetry.

Operative times and length of hospital stay are reduced. 
200

\section{Treatment of Poland Syndrome Mammary Hypoplasia with Autologous Fat Injection and Brava System: Preliminary Experience}

\section{Lancerotto, D. Del Vecchio*, F. Bassetto}

${ }^{\circ}$ Clinic of Plastic Surgery, University of Padova, Italy, ${ }^{\circ}$ Back Bay Plastic Surgery and Harvard University - MGH, Boston, USA

Introduction: Mechanical forces are being rediscovered as key element in cellular destiny control, in particular stretch. Indeed in Surgery mechanical forces have been empirically applied to induce tissue growth, i.e. in tissue expansion or distraction osteogenesis. A new application of this principle has recently been developed: Brava System (1). It consists of a two rigid cups device connected to a vacuum pump which can be applied to the breast to induce external tissue expansion. It seems particularly suitable for combination to the lipofilling technique, as its primary effects are an increase in soft tissues laxity and blood flow. This offers to the adipose tissue injected a more suitable environment for cells engrafting, promising an enhancement of the potentials of the technique (2).

Materials and Methods: A 16 y.o. patient with left breast hypoplasia and pectoral muscle aplasia in Poland syndrome underwent three cycles of 3 weeks Brava system mammary expansion + lipofilling +1 month post-operative Brava System expansion.

The treatment with the external negative pressure expansion plus autologous fat grafting is evaluated in terms of fat graft "taking" and mammary morphology. The application in the Poland Syndrome patient is compared with a case of additive mastoplasty in normal breast, and with previous cases treated with bicompartimental lipostructuring (3) alone.

201

\section{Complications in Breast Reconstruction}

M. Langella, P. Gigliofiorito, S. Tenna, B. Cagli, P. Persichetti

UOC Chirurgia Plastica Ricostruttiva ed Estetica,

Università Campus Bio Medico, Roma

Introduction: Complications in breast reconstruction may depend on many factors. Risk factors can be related to patient's habit, surgical procedures and adjuvant therapies. Classification is based on time of occurrence, reconstructive techniques or type of mastectomy.

Materials and Methods: Complications are divided in two groups (implants vs autologous tissue) and according to time of occurrence. Incidence rate is also considered. Early complications after implants placement include infections and hematomas, while capsular contractures, dislocation, deflation and rippling occur lately. In flaps reconstruction complications are infection, bleeding, hematoma, seroma, wound dehiscence, fat necrosis and skin necrosis. Late complications usually vary according to the type of flap.
Results: A review of minor and major complications is presented for both heterologous and autologous techniques, comparing authors' personal experience with the literature. Infections, capsular contracture and asymmetries were most likely registered in implant based reconstruction, while bleeding and partial flap necrosis occurred in autologous reconstruction.

Conclusion: Complications are related to major surgical procedures. However, correct preoperative evaluation of risks, appropriate selection of patients and related procedures, as training and knowledge of the techniques may keep the incidence lower.

\section{2 \\ Retrospective Analysis of 188 Consecutive Free Diep Flaps: Complications and Risk Factors}

B. Longo, R. Laporta, G. Cotroneo, P. Pugliese, M. Angelini,

UOD of Plastic Surgery, Sant'Andrea Hospital, "Sapienza" University of Rome, II School of Medicine

Introduction: Even though success rate in breast reconstruction with free DIEP flap has greatly improved partial flap necrosis (PNF) still occasionally occurs. Investigation of risk factors might elucidate mechanisms responsive for it.

Material and method: From May 2004 to October 2009, 188 breast reconstructions with free DIEP flap were performed on 183 patients; 166 flaps, including vascular zones I to III, were selected and retrospectively analyzed to assess complications rate and highlight risk factors. PFN $>1 \mathrm{~cm}^{2}$ was observed 27 times (16.2\%) and, since it was the most frequent complication, potential risk factors as tabagism, post-operative blood pressure, nulliparity, superficial vein drainage, medial or lateral perforator, employment of dedicated anesthesiologist and use of hypervolemic and antioxidant therapy (HAT) were analyzed using univariate and multivariate logistic regression analyses.

Results: Univariate analysis stated significance of nulliparity $(\mathrm{p}=0.024)$, not-dedicated anesthesiologist $(\mathrm{p}=0.014)$ and use of lateral raw $(\mathrm{p}=0.044)$. In multivariate model, nulliparity confirmed statistical significance $(\mathrm{p}=0.0302)$, whilst all other predictors showed variable correlations to PNF: tabagism increases PFN of $18.4 \%$, lateral row of $12.3 \%$, a not-dedicated anesthesiologist of $13.8 \%$, while the use of hypervolemic and antioxidant therapy represents a protective factor reducing PFN of $27 \%$.

Conclusions: Our study first proves that nulliparous patients show a statistically significant higher risk of PFN, possibly due to the lower caliber of perforators and smaller abdominal angiosome. The choice of a medial raw perforator, a constant and more accurate intra- and peri-operative care of patient perfusion by a dedicated anesthesiologist and a HAT might reduce partial necrosis incidence. 


\section{3}

Microvascular Bone Flap Prefabrication in an Animal Experimental Model : Clinical Future Implication

\section{P. Loschi, G. Spinzo, G. Boscaini, G. Petrella, L. Pacchioni, \\ F. Panico, G. De Santis}

Plastic Surgery-University of Modena and Reggio Emilia, Modena, Italy

Introduction: Despite major advancements in the field of biomaterials, autologous bone grafts are to be considered the gold standard for reconstructing extended bone defects. Our study was designed to develop a pre-fabricated bone flap in an animal model,starting from a predetermined-shape biocompatible scaffold, a vascular pedicle and different types of autologous stem cells.

Mat/Methods: 30 New Zealand rabbits were used and divided in three groups (10 rabb. each). In group 1 a biocompatible scaffold (xenogenic decellularized/deproteinized bone)was inset across the rabbit's femoral pedicle. In group 2(BM) the scaffold was inset and autologous bone marrow were added. In group 3 (ASCs)the same system was added with 1,5x106 autologous rabbit adipose-derived stem cells in hyaluronic acid matrix suspension. After 16 weeks all implants were harvested and analized.

Results: Histological analysis showed in group 1 implants an abundant connective tissue deposition, angiogenesis and bone resorption. In group 2 implants, osteogenesis was found and active osteoblasts were assessed. Group 3 implants were characterized by an intense inflammatory reaction.

Conclusions: $\mathrm{BM}$ are able to promote a faster osteogenesis process in this microsurgical model. ASCs may promote the same process with longer latency. Bone prefabrication may represent a possible reconstructive option in the future.

\section{4}

\section{Microsurgical Training in Italy}

S. Maxia, F. Farace, G. Fara, G.P. Muzzeddu, C. Rubino

Scuola di specializzazione di Chirurgia Plastica

Ricostruttiva ed Estetica, Università degli studi di Sassari

Introduction: Microsurgery is the one of most developed field of plastic surgery. Although it should be taught in all plastic surgery schools, not always trainee have a real microsurgical training.

The first difficult encountered by a microsurgical trainee is finding a microsurgical center with high quality reconstructive standard furthermore, this center should have the right equipment, authorized laboratories and facilities, rarely available due to legislative restrictions.

Materials and Methods: We performed a web search and a survey between trainee and plastic surgeon specialized in our school who performed the microsurgical training.
Results: The data we found shows that it is fundamental for doctors during the period of their residency in plastic reconstructive surgery, beside the learning of microsurgery with theoretical lessons, to take part in two kind of practical courses. On one side practical laboratory exercise on animals with surgical microscope, on the other side practical anatomical dissection on human corpse. We found currently available on web several courses of both kind, but unfortunately in Italy are not available courses of anatomical dissection due to lack of corpse given to science.

Conclusions: The aim of our work is to note a series of advices to help the young surgeon to choose easily its microsurgical training. In fact the theoretical basic knowledge should be supported by the practise through which can be acquired the automatisms and the techniques to perform microvascular anastomosis and pedicle dissection, to apply then to clinical practice.

\section{5 \\ VAC Therapy in the Treatment of Difficult Wounds in Patients with Controindications to Surgical Treaments}

\author{
M.P. Nejad, L. Negosanti, B. Tavaniello, D. Tassone, S. Palo, \\ R. Cipriani, P.G. Morselli, G. Zannetti \\ U.O. Chirurgia Plastica e Ricostruttiva - Policlinico S. \\ Orsola-Malpighi, Bologna
}

Study Aims and Objectives: The treatment of patients with difficult wounds often requires aggressive surgical debridement and subsequent reconstruction with skin grafts or flaps. These procedures usually require local or general anesthesia in patients with major comorbidity or poor general conditions, situations in which this may be contraindicated. We have successfully used negative pressure therapy with Vacuum Assisted Closure $\left(\mathrm{VAC}^{\circledR}\right)$ in selected patients. This has led to complete wound healing in a relatively short time and to the simplification of patient management of the patient.

Methods: We treated 4 patients with complex wounds in whom surgical treatment was contraindicated due to poor general conditions. In one case the important comorbidities contraindicated anesthesia, and in the second case the patient suffered from severe dementia, making it difficult to manage, the third patient was pregnant and refused to undergo surgical procedures and was the fourth patient was a minor. We used a VAC intermittent negative pressure $-125 \mathrm{mmHg}$. For the infant, a pressure of - $50 \mathrm{mmHg}$ and $75 \mathrm{mmHg}$ for 6 days for another seven days.

Results: Healing was obtained in a period ranging from 18 to 40 days and the result was satisfactory in all cases.

Conclusions: We present our experience to demonstrate the role of negative pressure therapy for treating difficult wounds when surgical procedures are not suitable or impossible to perform. 
206

\section{Technical, Functional and Aesthetic Evolutions After 10 Consecutive Years of Breast Reconstruction with Expander/ Mammary Prosthesis}

\author{
G. Petrella, A. Spaggiari, P. Loschi, A. Baccarani, \\ L. Pacchioni, A. Pedone, G. Spinzo, F. Panico, M. Pignatti, \\ G. Boscaini, G. De Santis \\ Università di Modena e Reggio Emilia. Azienda \\ Ospedaliero Universitaria Policlinico di Modena
}

Introduction: Breast cancer affects many women, but with advances in detection and treatment, survival rates have increased. Breast reconstruction can help to address the disfigurement and sense of loss that often follow mastectomy. The decision whether to pursue reconstruction and the choice of reconstructive strategy are individualized decisions that must take into account the patient's body characteristics, overall health, breast cancer treatment plan, and personal preferences. Options for reconstruction broadly include placement of breast implants. The aim of this study is to analyze the evolution of the prosthesis materials and shapes compared to the improvement of the morphological outcomes.

Materials and Methods: Over 560 cases of post oncological breast reconstruction done in the decade $2000-2010$. Prosthetic reconstruction of the breast, either with standard implants or as a staged procedure with tissue expanders followed by implants, has evolved into a reliable method for breast reconstruction. Advances in implant technology and biomaterials allow for low complication rates, good aesthetic outcomes, and consistent results.

Results: 19 (3,39\%) expanders replaced with flaps after radiotherapy, $10(1,78 \%)$ expander removed for infection and 5 $(0,89 \%)$ expanders substituted for valve failure. Moreover resolutions of particularly cases will be showed.

Conclusions: Although some may still consider autologous tissue reconstruction as the gold standard, today's prosthetic reconstructions play an equally important role in the reconstruction of the breast for many women.

\section{7}

\section{Madelung's Disease: A Case Report}

V. Pinto, D Tassone, L. Negosanti, B. Tavaniello, S. Palo,

P. Nejad, F. Zavalloni, C.M. Oranges, P.G. Morselli

Introduction: Madelung's disease, also known as multiple benign symmetrical lipomatosis, is a rare disorder characterized by the presence of multiple, symmetric, noncapsulated fat masses in the face, neck and other areas of upper extremities, with typical bulges in the neck.

Limitations in head and neck movements during eating and/ or speaking are main symptoms associated to benign symmetrical lipomatosis. In some cases severe clinical complications such as laryngeal or mediastinal compression or peripheral neurophaties are present. A clear association with alcohol abuse is reported in more than $90 \%$ of the patients. The ethiology of Madelung's disease remains unknown.

The transformation of Madelung's disease to a malignant tumor is extremely rare, but recurrence is the rule.

The condition does not spontaneously regress and medical treatment is not effective.

Gold standard is surgical treatment: open approach and/or liposuction.

As the benign nature of the lesions, surgical debulking with staged surgery, from simple liposuction to more massive skin incision and fat masses removal, is the treatment of choice.

Materials and Methods: CASE PRESENTATION: A 52 years old Caucasian man, affected by typical symptoms of Madelung's disease, has been treated. His major problem was severe reduction of neck motion and/or aerodigestive limitations, and dysmorphophysic appearance.

Results: According to literature, we performed multi-steps surgical treatment with substantial improvement in both functional and aesthetic appearance.

Conclusion: In our patient, affected by typical head and neck Madelung's lipomatosis, combined lipectomy and liposuction are a successful treatment, improving both aesthetic and functional appearance both psycho-social condition.

\section{8 \\ Port Disfunctioning in Becker Expanders as Cause of Volume-loss}

M. Sanna, F. Farace, M. Faenza, N. Sussarellu, G.V. Campus

Università degli studi di Sassari, Cattedra di Chirurgia

Plastica Ricostruttiva ed Estetica

Introduction: Several complications have been described for Becker Expanders, from infection to port upside down rotation and patient dissatisfaction (1). Usually, the injection dome is inserted into a subcutaneous pocket and the port is distant from the expander, connected by a thin tube system. Port removal is delayed from weeks to months at the end of the expansion process. Once the port system has been removed, under local anaesthesia, a retention device avoid implant deflation. We report a not yet described complication, the port malfunction.

Materials and Methods: A retrospective study was conducted on the last 80 consecutive breast reconstruction with a permanent tissue expander (100 implants), in over 2-years (20082009) in our department. All Complications were reported.

Results: 1 implant removed for a non-specific inflammatory reaction; 5 implant removed for infection; 1 implant removed non-specific deflation; 3 implant removed for immediate deflation after port removal (intraoperative).68 patients didn't want to remove their port device;

Conclusion: Our data shows 3\% of complication due to saline retention device malfunction. We suspect this device could work no longer than few months (all three cases were one year after implant). This causes a quick fast loss of volume, which lead 
to implant removal and replacement and should be counted as a major complication.

\section{9}

\section{Electrochemotherapy}

\section{G. Colombo, S. Ferraro, B. Scarsi, V. Ruvolo, M. Bormioli}

\section{Ospedale S.Corona Pietra Ligure}

The Electrochemotherapy with electroporation is a method using as drug either Bleomycin or Cisplatin and electric pulses to open trasmembranes pores of the neoplastic cells. The principle of ECT is based on the cells permeability increase by the application of electric fields induced by the instrument (CLINIPORATOR) on a small cutaneous area for a very short time. This method allows to deliver non permeant or poorly permeant chemotherapeutic agents into cell cytoplasm,increasing their dose-effect and reducing the toxicity. We can treat all cutaneous and subcutaneous tumour nodules of different histology: in particular cutaneous and subcutaneous bleeding and painful metastasis of solid tumours, metastasis in transit of melanoma also in areas previously treated with radiotherapy. The indications for this treatment are the reduction of size and number of cutaneous and subcutaneous metastasis, to make easier the following radiotherapy and surgery. Moreover it is an useful option when the local treatment is possible but the surgery is not possible or difficult. In our experience we had treated 17 patients, affected by secondary lesions of melanoma, squamous-cell carcinoma, basal-cell carcinoma, Kaposi sarcoma, 1 case of subcutaneous metastasis of bladder carcinoma and 2 cases of solar elastosi of damaged scalp.

\section{0}

\section{Different Strategies to Obtain a Good Result in DIEAP Flap Breast Reconstruction}

\author{
R. Sgarzani*, L. Negosanti*, V.Vietti*, R. Cipriani*, \\ P.N. Blondeel ${ }^{* *}$ \\ *Plastic Surgery Unit, Bologna University Hospital, \\ ${ }^{* *}$ Plastic Surgery Unit, Gent University Hospital
}

Background: The use of DIEAP flaps allows to achieve aesthetically pleasing mammary reconstructions, the same result can however be achieved with different strategies.

Materials and Methods: The procedure performed at Gent University Hospital was compared to the one performed at Bologna University Hospital. The following technical aspects were compared: pre-operative assessment, most frequent mastectomy pattern, recipient vessels selection, differences in donor site closure, flap modelling technique, average operative time, post operative flap follow-up and patient management, planned secondary procedures.

Discussion: These were the most significant differences noted: the use of internal mammary vessels makes it possible not to change patient position during operation reducing operative time and leaves a major freedom in flap shaping; the 3 steps principle makes flap modelling an easier and reproducible procedure; the use of CT scan, fibrin glue and absorbable skin stapler reduce operative time; spending additional time to mold and shape the umbilicus, dog-ears and contralateral breast provides an immediate nice result and avoids later corrections; lower flap outline leaves a lower scar on the abdomen, easy to hide also with low waist outfits.

Conclusion: Different procedures can obtain comparable aesthetically pleasing results, but some options help reducing operative times or make the results more reproducible.

\section{1}

\section{A Rare Case of Squamous Cell Carcinoma Arising in Chronic Perineal Hidradenitis Suppurativa}

\author{
D. Tassone, L. Negosanti, V. Pinto, P. Nejad, B. Tavaniello, \\ C. Gelati, R. Cipriani \\ Unit of Plastic Surgery, S.Orsola-Malpighi Hospital, \\ Bologna, Italy
}

Introduction: Hidradenitis suppurativa (HS) is a chronic inflammatory disease of the sweat glands and hair follicles which is rarely associated with squamous cell carcinoma (SCC). SCC usually occurs after 20-30 years after presentation of HS. Men are predominantly affected and the most frequent location for evolution in SCC is the perineal region. Actually in literature 40 cases have been reported with a mortality rate of $50 \%$ at 2 years.

Materials and Methods: We report a case of SCC arising on HS. The patient had a chronic HS in gluteal region for 20 years. In October 2008 appeared a sore and smelly ulcer in the same place. The lesion was biopsied and histologic examination revealed a moderately differentiated SCC. A CT scan was performed and revealed an infiltration in the plan underlying bone and the presence of suspicious inguinal lymphadenopathy.

Results: Surgical resection and reconstruction were performed by a team composed of general, orthopedic and plastic surgeons. The reconstruction of the defect was performed with local fasciocutaneous flaps (V-Y). Despite the extensive resection extended to the floor marrow, histological examination indicated residual disease on the deep margin. For this reason the patient underwent cycles of adjuvant chemo-radiotherapy. At 16 months after surgery the patient is alive without evidence of residual disease on CT/PET. It will still be kept under close follow-up cancer.

Conclusion: Aggressive surgical resection with reconstruction and adjuvant chemo-radiotherapy is a good therapeutic option in patients affected by SCC arising in chronic HS. 


\section{2}

\section{Pre-Surgical Assessment of Cutaneous Melanoma in Pregnancy Based on Clinical, Stereomicroscopy and Reflectance Confocal Microscopy}

\author{
B. Tavaniello*, I. Santi, M. Marengo ${ }^{\circ}$, S. Fanti, M. Medri $\square$ \\ I. Stanganelli $\square$, G. Zannetti* \\ *S.Orsola-Malpighi Hospital, Department of Plastic and \\ Reconstructive Surgery, Bologna University, S. Orsola- \\ Malpighi Hospital, Nuclear Medicine Department, Bologna \\ University, $\square$ Skin Cancer Unit, Istituto Tumori Romagna \\ (IRST), Meldola-Forli
}

Aim: We present a case of primary melanoma of abdomen occurred in a 36-year-old patient, diagnosed at the $8^{\text {th }}$ month of pregnancy.

Materials and Methods: Firstly the patient underwent to clinical and digital epiluminescence microscopy evaluation using stereomicroscopy.

The melanocytic lesion showed geometrical asymmetry, irregular borders, change in shape and colour, itch and a diameter of $4 \mathrm{~cm}$.

The malignant features were confirmed using a non invasive technique called reflectance confocal microscopy, whose result could be similar to an histological evaluation.

For these malignant features we performed a wide surgical excision and the sentinel lymph node biopsy.

In accordance with nuclear medicine physician, physicist and gynaecologist we performed a Standard 99m-Tc Nanocoll sentinel node lymphoscintigraphy paying attention to keep the baby's exposition to radiation within safety limits $(<0.3 \mathrm{mSv})$, so a reduced radioactivity of $18 \mathrm{MBq}$ was calculated and administered.

Caesarean section was performed at the $38^{\text {th }}$ week of pregnancy and during the same intervention the lesion was excised with $2 \mathrm{~cm}$-wide margins, and sentinel lymph-node biopsy was performed.

Results: Histopathological evaluation confirmed melanoma diagnosis (0.80 mm Breslow thickness, $1.0 \mathrm{~mm}$ regression, Mart-1 and HMB45 positive cells). Focally involved excision margins led to a surgical enlargement.

Histological examination of 5 lymph nodes from left groin and 4 from right groin and placenta resulted negative.

Conclusions: In case of suspected cutaneous melanoma occurring in pregnancy, a multidisciplinary approach is suggested, taking into account surgical-linked risks. The interactive multimodal diagnostic non invasive steps were closely related to the decision for the wide excision and node procedure before the histopatological stadiation.

\section{3}

\section{Electrochemotherapy for Primary or Metastatic Skin Tumours: A Single Institution Experience}

\author{
B. Tavaniello*, A. Ceccone ${ }^{\circ}$ S. Palo*, D. Tassone*, \\ L. Negosanti, P. Nejad ${ }^{*}$, V. Pinto*, G. Zannetti* \\ ${ }^{*}$ S.Orsola-Malpighi Hospital, Department of Plastic and \\ Reconstructive Surgery, Bologna University, 'S. Orsola- \\ Malpighi Hospital, Radiotherapy Department, Bologna \\ University
}

Purpose: To evaluate efficacy of electrochemotherapy in term of complete response (CR), partial response (PR) and local control rate using i.v. bleomycin (BLM) or intralesional cisplatin (CDDP) in patients affected by cutaneous and subcutaneous melanoma metastases and other primary or metastatic tumours.

Materials and Methods: From July 2006 to March 2010, 26 patients (pts) and 123 lesions were treated according to European Standard Operating Procedurs of Electrochemotherapy (ESOPE) with ECT. After local anaesthesia the electric pulses were applied to the tumour to increase bleomycin or cisplatin delivery into tumour cells. There were in-transit metastases of melanoma: 22 pts (112 nodules), skin metastases from breast cancer: 1 pt (5 nodules), basal cell carcinoma: 1 pt ( 1 nodule), squamous cell carcinoma of the vulva: 1 pt ( 1 nodule), squamous cell carcinoma of the head skin $: 1 \mathrm{pt}$ (4 nodules). In 6 pts we used intralesional CDDP and in other pts (20) BLM i.v..

Mean age was 78 years. Median follow-up was 14.1 months (range 27 days-44 months).

Results: After a median follow-up of 14.1 months, $3 / 26$ pts were lost to follow-up, 15/23 (65.2\%) pts are alive in CR, in 3/23 pts we observed a progression of systemic disease after 2 months and $5 / 23(21.7 \%)$ pts are in PR; the local control rate is $86.9 \%$. No significant differences in response we observed comparing to the two drugs. No local and systemic toxicity was documented and the tolerance was adequate.

Conclusions: ECT is an effective local treatment for patients affect by cutaneous and subcutaneous tumours nodules of melanoma and other different malignancies. ECT represents a good alternative to surgery or radiotherapy to improve the local control of skin metastases and it is an excellent possibility of re-treatment without inducing a more systemic toxicity effect. 
214

\section{Cell Mediated Immune System Hyperactivation and Complications in Surgery. Is There a Correlation?}

F. Toia, C. Liuzza, S. Meraviglia, R. Grassi, S. D’Arpa, F. Dieli

Chirurgia Plastica Ricostruttiva ed Estetica, Dipartimento

di Discipline Chirurgiche ed Oncologiche, Università degli

Studi di Palermo, Dipartimento di Biopatologia e

Biotecnologie mediche e forensi, Università degli Studi di

Palermo

Introduction: The role of the immune system in wound healing is multifaceted and cell-to-cell interactions may affect healing in several ways, many of which are probably still unknown. The case of a 19-year-old girl who, after multiple postsurgical complications, was found to have a transient immune system hyperactivation is reported.

Materials and Methods: A 19-years-old woman with a breast asymmetry, previously treated with breast augmentation and contralateral reduction mammaplasty, had her implant removed and she came to our attention seeking autologous reconstruction. A free DIEP flap was performed and, again, wound breakdown and liponecrosis ensued at both the donor and recipient sites. Bacterial cultures were negative. Three months after the operation, a second attempt at autogenous breast volume enhancement was done with lipofilling. Fat necrosis occured again. At this point immunological consueling was requested.

Results: Microbiologic and immunologic tests did not reveal any infectious disease. Immunologic studies revealed a polyclonal hyperactivation of CD4+ and CD8+ T-lymphocytes that showed higher rate of apoptosis and mitosis. Immunological profiles returned to normal within 3 months after surgery.

Conclusions: An unusual hyperactivation of the cell mediated immune system, while fat necrosis was ongoing, coupled with return-to-normal after healing, led us think that the cell mediated immune system hyperactivation could have caused a sort of self aggression in the surgical sites. However, as this is only an incidental finding, further studies are needed in order to prove any correlation.

\section{5}

\section{The Bilobed Flap in Nasal Reconstruction}

M. Valente, M. Grieco, P. Bisceglia, F. Lembo,

A. Campanale, L. Annacontini

Plastic and Reconstructive Surgery Department, University of Foggia - Italy, Head: Prof. Aurelio Portincasa, EBOPRAS Fellow

Introduction: Zitelli's bilobed flap offers the ability to aesthetically reconstruct difficult defects in the distal nasal area. However, the Zitelli bilobed flap may sometimes result in nasal alar retraction which is likely to the result of excessive tension upon closure of the primary lobe and primary defect.
Methods: This flap was employed for distal nasal reconstruction in 70 patients following excision of malignancy from January 2005 to December 2009. The series includes 24 females and 46 males ranging in age from 32 to 83 years. Laminar drainage used in less than $8 \%$ of all cases.

Results: The results of the reconstruction were satisfactory: there was good nasal contour and appropriate symmetry of the nasal tip and no alar retraction. Only $4 \%$ of patients had a partial secondary healing.

Conclusion: The flap was viable in all cases and the aesthetic outcome satisfactory. The rotation fold dog ear is eliminated. In planning the Zitelli flap the length of its lobes (proximal and distal), should be equal to the defect; the width of the proximal lobe, compared to the defect, and the distal lobe compared to the proximal lobe should be progressively narrower.

\section{6 \\ 'Cellular Therapy' Through Lipostructuring; the Role of Adipose-derived Adult Stem Cells in 'regenerative Surgery' Processes: Outcomes Over the Past 3 Years}
L. Annacontini, M. Valente, M. Rucci, D.N. Massariello,
A. Campanale, A. Maiorella
Plastic and Reconstructive Surgery Department, University of Foggia - Italy, Head: Prof. Aurelio Portincasa, Ebopras Fellow

Background: Adipose tissue grafting (lipostructuring) is regularly used for volume corrections due to congenital malformations and/or syndromes, burns, trauma and after oncologic and/or aesthetical procedures. More recently the identification Adipose-Derived Adult Stem Cells in the grafted adipose tissue, clarified why skin trophism and healing processes improve dramatically after lipostructuring, compared to other surgical reparative procedures.

Materials and Methods: 19 patients, from January 2006 to June 2009 underwent lipostructuring according to the Coleman technique (12F, $7 \mathrm{M}$, range $13-58$, mean age 36 years) for pathologic scars, congenital malformation and volume deficiency of the face ( 4 cases), breasts ( 6 cases), thorax ( 2 cases), abdomen (4 cases), upper and inferior limbs (3 cases). In all cases a short term profilaxis was used. Mean hospital stay was 3-5 days. Follow up performed at 3,6 and 12 months after the operation with pinch and punch tests.

Results: Adipose-Derived Adult Stem Cells presence in adipose tissue makes of lipofilling a "cellular therapy": the stem cell identification with histology, citologic and ultrastructural caracterization, immuno-histochemical identification confirmed the potential of this surgical option. Long-term results highlighted how a regular healing process followed the lipofilling with encouraging results in term of skin trofism, disappearance of symptoms like pain, parestesy, disestesy, allowing the resolution of volume losses with an aesthetic improvement - in selected cases - compared to other surgical procedures. We did not observe complications excepted the necessity to repeat the lipofilling because a 
mean of $40-50 \%$ of injected adipose tissue will be resorbed after 6 to 12 months.

Conclusions: Lipostructuring appears to be a safe, easy, repeatable technique capable to improve tissue healing, cells "regeneration", bothersome symptoms, useful in many different clinical conditions.

\section{7 \\ Dye Laser in the Treatment of Hypertrophic Scars}

\author{
M. Rucci, M. Valente, M. Grieco, D.N. Massariello, \\ A. Boccuzzi, G. Giulia, L. Annacontini \\ Plastic and Reconstructive Surgery Department, University \\ of Foggia - Italy, Head: Prof. Aurelio Portincasa, EBOPRAS \\ Fellow
}

Introduction: Pathological scars affect the $3 \%$ of world population. Nowadays the treatment includes medical devices such as silicone sheets and local infiltration of corticosteroids. Authors present their experience in the treatment of pathological scars with the application of Dye Laser Dermobeam $2000(\lambda 585 \mathrm{~nm})$.

Material and Methods: 2004- 2009: 40 patients (18 F, 12 $\mathrm{M})$, range 6-55, with hypertrophic scars throughout the body were treated with Dye Laser. The intensity, width and duration of the spots were modulated in relation to different anatomical areas, skin type, age and sex of the patient. Patients underwent 8 treatments each, every month, followed by daily application of hydrating and soothing products. We evaluated: height / width (A / S), elasticity / softness (E/M), appearance (AE), in a scale from 0 to 3 .

Discussion: The hypertrophic scar is characterized by abnormal and irregular deposition of collagen fibers, supported by excessive neovascularization. Dye Laser is a valuable tool in their treatment because induces coagulative necrosis, destruction of collagen fibers, dissociation of disulfide bonds and subsequent realignment of collagen fibers.

Results: After the 4th treatment 35 patients (83\%) showed flattening and whitening of the hypertrophic scar with an improvement of skin elasticity. 30 patients reported a significant improvement in term of itch and pain.

Conclusions: Next to a medical treatment (elastocompression and / or corticosteroids) and surgery, Pulsed Dye Laser Dermobeam 2000 appears to be a valid option in the treatment of hypertrophic scars.

\section{8}

\section{Orbital Floor Reconstruction: PDS or Titanium Mesh?}

L. Fedele, A. Boccuzzi, P. Bisceglia, M. Valente, A. Campanale, L. Annacontini, D. Parisi

Plastic and Reconstructive Surgery Department, University of Foggia - Italy, Head: Prof. Aurelio Portincasa, EBOPRAS Fellow

Introduction: The orbit is particularly susceptible to fractures because of its exposed position and thin bones, and external impact to this area can cause orbital floor fractures. Indications for surgery are: persisting diplopia, increased orbital pressure, enophtalmos, visual impairment, hypoaesthesia of infraorbital nerve and severe dislocation of bone fragments. There aren't universal guidelines for timing and materials using for reconstruction.

The purpose of this study is the evaluation of accuracy of orbital floor reconstruction in our experience.

Materials and Methods: From January 2004 to January 2009 twelve patients ( 4 \% and $80^{*}$; range $18-52$ yrs; mean age 35 yrs) with orbital floor fractures have been treated with alloplastic materials (PDS: 5 pts; titanium mesh: 7 pts). One year after trauma we compared reconstructed orbital floor to unaffected side using 3D-CT scans; enophtalmos was measured using Hertel index.

Results: Reconstruction with titanium mesh was very precise and only one patient $(14,3 \%)$ had enophtalmos, while after use PDS in two patients $(28,6 \%)$ orbital floor was lower (vertical dimension) compared to unaffected side, and consequently they have enophtalmos and diplopia.

Conclusions: Nowadays PDS foils and Titanium mesh are the ideal orbital floor repair materials. Proper selection and use of this materials will usually ensure surgery success. Orbital floor reconstruction with Titanium mesh was more precise and with fewer complication. In fact we believe that titanium mesh should be used in fractures with large defects, while PDS foils only where it's need of orbital volume restoration.

\section{9}

\section{Our Experience of Pectoral Major Flap in Neck Reconstruction}

\section{F. Lembo, P. Bisceglia, A. Boccuzzi, L. Annacontini, A. Campanale, D. Parisi \\ Plastic and Reconstructive Surgery Department, University of Foggia - Italy, Head: Prof. Aurelio Portincasa, EBOPRAS Fellow}

Introduction: Radical ablative surgery (with or without RT) is the gold standard treatment of head and neck cancer. The development of the regional flap is the most significant advance in reconstruction of this areas.

Many flaps have been tried for reconstruction of neck surgical defect after severe oncological demolition for malignant tumor. 
The purpose of this study was to prove that the use of PM flap, muscular or myocutaneous, is a good strategy for management of these wounds.

Materials and Methods: From January 2005 to January 2009 twenty patients ( $8 \%$ and $120^{\prime}$; range $48-72$ yrs; mean age 65 years) with massive malignant tumor of the neck have been treated with wide demolition and radical lymphoadenectomy and reconstruction with PM, muscular or mc flap. Short term prophylaxis always used. 16 donor areas were directly closed while 4 with skin graft. One to 5 yrs follow up observed.

Results: We observed: 2 hematomas, 1 seroma, 1 partial skin necrosis but no flap necrosis or infection. Mean postoperative healing time was 38 days (range: $25-58$ ). Strength preservation of superior limb in all patients. 2 patients died during the follow-up for tumor progression.

Conclusions: Essential for an optimal outcome are: preoperative planning; accurate patient selection; surgeon experience and adequate postoperative care. Only in this way the PM flap is a versatile flap which ensure a good reconstruction of neck after oncological demolition, with lower complication rate and enhancement of patient compliance.

\section{0}

\section{Integra Interdisciplinary Indications}

L. Annacontini, M. Valente, M. Grieco, M.P. Bisceglia, D.N. Massariello, D. Parisi

Plastic and Reconstructive Surgery Department, University of Foggia - Italy

Head: Prof. Aurelio Portincasa, EBOPRAS Fellow

Introduction: INTEGRA $^{\circledast}$ is a temporary bilaminate membrane system used as permanent dermal layer regeneration where a superficial silicone sheet is substituted with a split-thickness autograft.

Material and Methods: INTEGRA ${ }^{\circledR}$ indications are multiple: vascular ulcers, burns, post oncological or post traumatic reconstructions, congenital malformations.

From January 2004 up to day 54 pazients (36F and 18M, 3-82 yrs, mean age 54 ) treated: 38 post-surgical cases ( 31 post oncological demolition, 7 post traumatic cases), $5 \mathrm{pz}$ affected by vascular ulcers, $4 \mathrm{pz}$ after burns, $6 \mathrm{pz}$ with retracting scars and $1 \mathrm{pz}$ affected by the Proteus Syndrome.

The INTEGRA ${ }^{\otimes}$ is accurately shaped on the lesion, fixed with the Appose, dressed with Vaseline and sterile gauzes, covered with elastic bands (T0). New medications occurred at T7, T14, T21. After 4 weeks a split-thickness autograft $(0.16 \mathrm{~mm})$ is performed.

Results: Wound bed preparation with the INTEGRA ${ }^{\star}$ results optimal for the subsequent split-thickness autograft. Many are the advantages: better morfo-functional result compared to the simple thin skin graft; similar result to the total skin graft; minor donor site damage; minor trauma if compared to the flaps; possible use in patients with exposed structures (tendons and bones, in selected cases); readily available; grafting can be delayed until the patient is stable and donor sites are plentiful. "Disadvantages" are: minimum training required; accuracy of the procedure; serial clinical controls; patients selection; two-steps procedure.
Conclusions: Most of our patients (near to 65\%) obtained satisfactory morfo-functional results. With no doubt in selected patients INTEGRA ${ }^{\circledR}$ can be considered a valid, safe, option to more sophisticated procedures like local and/or microsurgical flaps.

\section{1}

\section{Rizhoartrosis Two Years of Experience}

\section{P. Bisceglia, M. Rucci, M. Valente, F. Lembo, L. Annacontini, A. Campanale, D. Parisi}

Plastic and Reconstructive Surgery Department, University of Foggia - Italy, Head: Prof. Aurelio Portincasa, EBOPRAS Fellow

Introduction: Rizhoartrosis involved the first metacarpus and the trapezius, compromising functionality of the first finger both in term of strength and prehensile capability.

The first symptom is a heavy increasing pain at the basis of the first finger, with the swelling of the tissue around the articulation, due to the first metacarpus incomplete dislocation.

The diagnosis is aimed through standard X-ray exam in frontal and latero-lateral projection.

According to the clinical classification it is possible to distinguish different stages: the first is treated with a conservative method, while the last three stages need a surgical treatment.

Materials and Methods: From January 2008 to January 2010 we compared 15 cases of rizohartrosis, 9 treated with Ceruso modified suspension arthroplasty and 6 with the introduction of a Pyrocarbonic Prothesis. The choice was made according to the age, sex and working activity of the patients.

The post operative evaluation included: post-operative recovery, pain (estimate with VAS scale), strength and prehensile function (estimate with DASH questionnaire).

Discussion: According to our experience and to postoperative evaluation, both the Ceruso Arthroplasty and the introduction of Pyrocarbonic Prothesis lead to good

Results: All patients showed a reduction of the pain, and regained strength and prehensile capability. However, the introduction of the Prothesis was restricted to those patients that not required a complete functional recovery (old patients, no manual working activities).

Conclusion: The treatment for Rizhoartrosis includes different Surgical techniques, all valuable, which main aim is reduction of the pain. The choice is influenced by patients' characteristics and by his needs to retrieve the complete functionality of the hand. 
222

\section{Glandular Rearrangement for Upper Quadrants Reconstruction in Breast Conserving Therapy}

\section{A. Campanale, L. Annacontini, M. Rucci, F. Lembo, M. Valente, A. Maiorella, D. Parisi \\ Plastic and Reconstructive Surgery Department, University of Foggia - Italy, Head: Prof. Aurelio Portincasa, EBOPRAS Fellow}

Introduction: Conservative treatment of breast cancer is widely accepted as the gold standard for early-stage tumors for its oncological safety.

The Authors present a new surgical procedure which avoids radical mastectomy to women affected by early breast cancer in the upper quadrants and moderate hypertrophy.

Material and Methods: From April 2007 to August 2009, 13 patients with early breast cancer underwent upper quadrantectomy and immediate reconstruction using cutaneous-glandular flaps. The mean age was 43 years. Cancer was diagnosed by FNAB: 5 in the medial and 8 in the lateral upper quadrant. All had moderate hypertrophy and second-degree ptosis. None was smoker, diabetic, or affected by obesity or microvascular disease, none had undergone neoadjuvant chemotherapy or radiotherapy. Aesthetic and functional outcomes were evaluated.

Results: Nine were ductal, 5 lobular carcinoma; all in stage up to T1b. Mean weight of resection was $76 \mathrm{~g}$. Mean operative time: $115 \mathrm{~min}$. All patients healed without complications within 15 days. There were no recurrences or late complications during the follow-up. No scars revision were require. Satisfaction was high for aesthetic and functional outcomes.

Discussion: In 2009 a Modified Wise-Pattern Reduction Mammaplasty was proposed as a New Tool for Upper Quadrantectomies. In our Department we use it in all patients with moderate hypertrophic and ptotic breasts but we don't remove further glandular tissue as previously described. Radiotherapy can be performed without side effects. The décolleté is free from any scars.

Conclusion: The technique allows good and long-lasting cosmetic and functional results only with glandular rearrangements avoiding mutilating surgery.

\section{Thoracic Surgery}

\section{3 \\ Management of Tracheo-bronchial Foreign Bodies in Children: Our Experience}

\author{
D. Angela, T. Francesca, P. Ondina, L. Domenico* \\ S. Francesco*, L. Michele
}

Cattedra di Chirurgia Toracica, Università degli Studi di Bari, " Cattedra di Chirurgia Toracica, Università degli Studi di Foggia

Introduction: Foreign bodies (FB) inhalation in children is frequent and potentially lethal. Tracheo-bronchial FB are generally removed by rigid bronchoscopy (RBS); fiberbronchoscopy (FBS) is a complementary technique; thoracotomy is reserved for cases of failed endoscopic extraction. We report our experience in the management of tracheo-bronchial FB in children.

Methods: From January 1998 to April 2010, 125 children (mean age 2.8 years, range 6 mo- 8 ys) were referred to our Institution for suspected FB inhalation. All patients underwent explorative FBS through orotracheal tube, under general anesthesia; FB were actually found in 47 cases (38\%) and removed with dedicated forceps in RBS (35 cases) or combining RBS and FBS ( 9 cases; in 6 by Dormia basket). After unsuccessful endoscopic extraction, 2 FB were removed by bronchotomy through thoracotomy. In a FB embedded at the glottis level, tracheotomy was required below the $\mathrm{FB}$, which was then extracted by ORL.

Results: All FB were quite easily, successfully removed; one patient only, referred to our ICU intubated and in coma for acute respiratory failure after inhalation, despite successful extraction, later died.

Conclusion: Tracheo-bronchial FB removal in children is a high-risk procedure. An optimal cooperation between surgeons and anesthesiologists is necessary to successfully perform this procedure with minimal complications.

\section{4}

Fluoroscopy-aided Thoracoscopic Surgery of Pulmonary Nodule Localized with Platinum Coil (FATS)

\author{
A. Droghetti, M.C. Bottoli, A. Tagliani*, C. Bna*, \\ M. Beccaria , G. De Donno, C. Sturani, G. Muriana. \\ Struttura Complessa di Chirurgia Toracica, ${ }^{\circ}$ Struttura \\ Complessa di Pneumologia e UTIR, * Struttura Complessa \\ di Diagnostica per Immagini, Azienda Ospedaliera "C. \\ Poma" di Mantova
}

Background: Videothoracoscopic Surgery (VTS) resection of small pulmonary nodules $>10 \mathrm{~mm}$ deep to the visceral pleura 
fails to locate the nodule and requires conversion to open thoracotomy in two thirds of cases.

We report our three years experience of the procedure in which a "Platinum Coil" was deployed adiacent to the nodule under preoperative CT that enable to detect and excise nodule in thoracoscopy by fluoroscopic guidance.

\section{Patients and Methods:}

- Forty-one patients with peripheral pulmonary nodules $<20$ $\mathrm{mm}$ diameter was treated from 2005 to 2010. Anatomical preoperative localization of the target lesion by 1-3 mm layers HRCT with three-dimensional percutaneously positioning of Platinum Coils.

- Resection of the noduleguided by fluoroscopy and videothoracoscopy.

- Histology examination was performed immediately after resection.

\section{Results:}

- All nodules was successfully resecate with desease-free margins.

- Three minimal pneumothoraces didn't require chest drain

Conclusions: Fluoroscopy-Aided Thoracoscopic Surgery with Platinum Coils help the thoracic surgeon to detect small lesions avoiding thoracotomy, is a safe and effective.

\section{5}

\section{Videothoracoscopic Implantation of the Left Ventricular Pacing Lead for Cardiac Resynchronization Therapy}
A. Droghetti', M.C. Bottoli', P. Pepi, A. Fusco3,
C. Bonadiman², R. Casarotto*, W. Mosaner*, A. Vicentini3,
G. Muriana?.

'SC di Chirurgia Toracica, ${ }^{\circ}$ SC Cardiologia e UTIC - Azienda Carlo Poma - Mantova, ${ }^{2} \mathrm{SC}$ di Chirurgia Generale, ${ }^{3} \mathrm{SC}$ di

Cardiologia, *SC di Anestesia e Rianimazione - Casa di

Cura Pederzoli - Peschiera del Garda

Background: The transvenous placement of the left cardiac pacing leads is sometimes impossible for the absence of satisfactory venous access or unfavorable anatomy of the coronary sinus, unstable position or diaphragmatic twitch.

The innovative positioning tool "FastacFlex" allows epicardial anchoring by videothoracoscopy.

Patients and Methods: 13 patients, median age 64 years, underwent implantation of the left ventricular epicardial pacing lead.

All procedures were performed with 3 ports VTS $(5 \mathrm{~mm}, 10$ $\mathrm{mm}$ and $15 \mathrm{~mm}$ ) on the left chest wall.

Results: The epicardial lead was successfully placed in all patients. The median procedure time was 63 minutes.

Final measurements showed median threshold values of 1,3 $\mathrm{V} / 0,5 \mathrm{~ms}$.

Neither complications nor dislocations were observed.

The chest drainage was average removed after 36 hours.

Median length of hospital stay was 3 days.
The average follow-up was 472 days with symptomatic benefit with reduction in New York Heart Association score from III preoperatively to II postoperatively.

Conclusions: The VTS placement of the epicardial pacing lead is safe, feasible and efficacious with comparable DFTs with conventional transvenous implant.

\section{6 \\ Closure of Right Bronchopleural Fistula with Amplatzer Septal Occluder}

\author{
A. Droghetti, M.C. Bottoli, A. Comel, C. Lettieri, C. Sturani, \\ G. Muriana \\ SC Chirurgia Toracica, SC Pneumologia e UTIR, SC \\ Cardiologia e UTIC, SC Anestesia e Rianimazione - \\ Azienda Ospedaliera Carlo Poma - Mantova
}

We describe three treatments for endoscopic closure of right bronchopleural fistula after pneumonectomy with Amplatzer septal occluder (autoespandible device with a double-disc Nitinol wire mesh with polyester fabric inserts), normally used for occlusion of atrial septal defects.

The procedure was performed by double-checking with bronchoscopy and videothoracoscopy through thoracostomy.

The Amplatzer septal occluder introducer was pushed forward through the rigid bronchoscope until you pass through the fistula entering the pleural cavity where the distal disc was released, then retracted by the mounting system until completely occlude the fistula orifice outside.

The proximal disc is then released in the bronchial stump, until complete occlusion thereof.

The procedure, rapid and repeatable, lasted an average of 40 minutes; upon awakening the patient was transferred to the ward and subsequently discharged without complications.

The follow-up show progress with granulation tissue covering the endobronchial device, that is complete after 5-6 months, with complete closure of the fistula.

This treatment, for the first time performs in Europe, confirms the evidence in bibliography of safety and surprising effectiveness of this technique for the closure of BPF.

\section{7}

\section{Limited Upper Sternotomy in General Thoracic Surgery}

\section{F. Antonacci, K. Kawamukai, S. Fortiparri, B. Bonfanti, M. Boaron \\ Dpt of Thoracic Surgery, Maggiore and Bellaria Hospitals, Bologna, Italy}

Background: To evaluate the current employment of limited upper sternal split in the practice of general thoracic surgery on the bases of our 13 years experience with this surgical approach. 
Methods: Retrospective review of clinical files of all patients operated on through limited upper sternotomy between 1995 and 2008.

Results: 138 consecutive patients were operated on through limited upper sternotomy. Thymus and thyroid surgery represented the main indications for this approach $(n=68$ and $n=36$, respectively). In the remaining cases indications included assessment of residual disease after chemotherapy for Lymphoma $(n=14)$; tracheal surgery $(n=8)$; excision of nodal mediastinal metastasis $(n=3)$; cervical spine surgery $(n=3)$; lung cancer staging $(n=2)$; combined access for esophageal cancer $(n=2)$ and parathyroid adenoma $(n=2)$. No operative mortality was recorded. Complications occurred in 10 cases.

Conclusion: Upper sternal sternotomy represents a fully satisfactory surgical approach for many mediastinal operations. Its limited invasiveness, good cosmetic results, and satisfactory exposure should suggest its employement whenever possible and safe.

\section{8}

\section{Single-Staged Laryngotracheal Resection and Reconstruction for Benign Strictures in Adults}

\author{
B. Lucia, M. Giuseppe, S. Nicotra, M. Loy, F. Di Chiara, \\ A. Rebusso, R. Federico \\ Department of Cardiac, Thoracic and Vascular Sciences, \\ Thoracic Surgery Division, University of Padova, Medical \\ School
}

\section{3rd Classified - SPIGC Award Session, Thoracic Surgery}

Objective: Laryngotracheal stenosis represents a challenging problem requiring a complex management. Surgical approach allowing permanent chance of airway restoration is the preferred treatment. Purpose of study is to evaluate short and late outcome following laryngotracheal resection-anastomosis.

Methods: From 1994 to February 2010, 48 patients (34 males and 14 females, median age 33 years) underwent surgery. Causes of stenosis were postintubation or posttracheostomy injury in 39 cases and idiopathic in 9. Pearson's technique was used for anterolateral crico-tracheal resection $(n=29)$ and Grillo's technique providing a posterior flap of tracheal membranous for circumferential stenosis $(n=19)$. Since 1998, in 30 cases we modified the techniques using a continuous $4 / 0$ polydioxanone suture for posterior part of anastomosis.

$40(83 \%)$ patients received a total of 79 preoperative interventions including laser $(n=25)$, tracheostomy $(n=36)$ and endotracheal prosthesis $(n=18)$.

Results: No perioperative mortality was recorded. Three (6.25\%) patients developed major complications (2 fistulae and 1 early stenosis) that required a second surgical look. We had 25 minor complications in $18(37.5 \%)$ patients: granulation tissue $(n=6)$, anastomotic oedema $(n=7)$, temporary vocal cord dysfunction $(n=8)$, wound infection $(n=3)$, anastomotic fibrin deposit $(n=1)$. Late results were excellent in $37(77 \%)$ patients, good in 9 (19\%), satisfactory in $1(2 \%)$, unsatisfactory in $1(2 \%)$.
Conclusion: Single-staged laryngotracheal resection is a demanding operation, but can be performed successfully with acceptable morbidity in specialised centres.

The continuos suture in posterior part of anastomosis simplifies the procedure without technique-related complications.

In our experience this procedure guaranteed excellent and good long-term results in more than $90 \%$ of patients.

\section{9}

Penetrating Cardiac Injuries: Our Experience

J. Brandolini, M. Salvi, G. Grani, A. Lucchi, P. Berti, F. Vandi, G. Garulli

U.O. Chirurgia Generale-Toracica, Ospedale di Riccione (Rimini)

Introduction: A review of the literature shows as only the $25 \%$ of the patients with penetrating cardiac injuries can survive before reaching the hospital. The prognosis of these patients depends mostly in the intervening time from the traumatic event and the surgical treatment, as well as the size, the place of the myocardial injury and the presence of a cardiac tamponade.

Materials and Methods: From 2005 to 2009 three patients with penetrating wound of the myocardium by cold steel have been recovered into our U.O. The doubt of a myocardial injury in the presence of an hemorrhagic shock and marks of cardiac tamponade, was well confirmed by an urgent echocardiographic test.

Results: For each patient it was chosen a sternotomic approach together a pericardiectomy and a Prolene interrupted suture of the myocardial wound. For one case it was done a service left antero-lateral thoracotomy because of the weapon and the presence of an associated hemo-pneumothorax. The post operating course was without complications for the three cases, with a stay in hospital of about 12 days.

Conclusions: From literature revision and from our experience, we confirm that the quick transport of the patient to the nearest hospital, an early diagnosis and an aggressive and immediate surgical treatment are essential for the survival of these patients. The echocardiographic test represents today the best research of the choice in the evaluation of the patients with penetrating cardiac injuries, because it is repeatable and not invading.

\section{0}

\section{Catamenial Pneumothorax: Our Experience}

J. Brandolini, M. Salvi, G. Grani, M. Alifano, A. Lucchi, P. Berti, F. Vandi, G. Garulli

U.O. Chirurgia Generale-Toracica, Ospedale di Riccione (Rimini)

Introduction: Thoracic entrometriosis is considered a rare clinical condition. The catamenial pneumothorax is the most frequent condition; it represents a rare importance of spontaneus 
and relapsing pneumothorax, manifesting itself in a synchronism manner together the menstrual period.

Its etiology isn't very clear till today. The objective of this study is to value the clinical traits and the therapeutic strategy of the patients affected by catamenial and not-catamenial pneumothorax subordinate to the endometriosis.

Materials and Methods: 114 young women have had in a six years period a surgical treatment for pneumothorax ; in 28 cases $(24,6 \%)$ it was diagnosed a catamenial pneumothorax, together right pleural anomalies in 27 cases. In 22 cases it was seen diaphragm defects. All the patients have had a video assisted surgical treatment by the resection of the dystrophic zones of the pleural endometriosic foci and following pleaural symphysis to present the recidivations.

Results: The anatomopathological study has showed the presence of endometrical focus, mainly of the diaphragm in 18 patients of 28 with catamenial-pneumothorax and in 11 of 86 with non-catamenial pneumothorax. The percentage in the long run recidivations in patients with catamenial pneumothorax and not-catamenial pneumothorax tied to the endometriosis has been respectively of the $32 \%$ and $27 \%$.

Conclusions: Our experience shows as the catamenial and the not-catamenial pneumothorax secondary to the endometriosis represent a good percentage of the pneumothorax good for the surgical treatment; considering the high rate of recidivations, the best treatment should be multidisciplinary combinating the surgical treatment with the hormonal therapy.

\section{1}

\section{The Benign Metastatic Leiomyoma: A Case Report}

J. Brandolini, M. Salvi, G. Grani, A. Lucchi, P. Berti, F. Vandi, G. Garulli

U.O. Chirurgia Generale-Toracica, Ospedale di Riccione (Rimini)

Introduction: The benign metastatic leiomyoma (BML) is a pathology where the lung represents the most touched extra-uterine organ. The pulmonary metastatic leiomyoma is a rare benign tumor of the smooth musculature that concerns the peripheral zones of the lung or the walls of the windpipe and the bronchus. Generally we can see carrier women of uterine-leiomyoma, but in women who had undergone hysterectgomy, in a reproductive age, for the same reason.

Materials and Methods: We report a case of a young woman of 43 years with a pregressive history of hysterectomy for uterine-fibroma, arrived to our study to have a surgical treatment of the left hemicolectomy for colonic neoplasia, and a casual discovery during the staging pre-treatment, of a plury bilateral pulmonary neoformations compatible with a benign leiomyoma.

Results: A wedge biopsy revealed the pulmonary nodule consisting of branching glandular structures surrounded by abundant smooth muscle cells. Immunohistochemical studies confirmed diagnosis.

Conclusions: The benign metastatic leiomyoma represents a rare clinical entity and in the most cases is considered as a benign lesion. This tumor results to be ormonal-sensitive even if an important percent of the cases, in spite of the treatment, is unchanged. The presence of pulmonary metastatic in women affected by uterine-leiomyoma or with a previous hysterectomy has to lead to do an hystological test of the pulmonary lesion to exclude a malignant pathology. Considering the few cases described in literature, the use of the chemoprophylactic therapy to prevent relapses after the surgical resection, remains much discussed yet.

\section{2 \\ Use of Autologous Platelet Leucocyte- Enriched Gel for Treatment of Persistent Air-Leaks After Pulmonary Resection

\author{
C. Carillo, T. De Giacomo, F. Venuta, D. Diso, M. Anile, \\ V. Liparulo, C. Ricella, M. Patella, F. Francioni, G.F. Coloni \\ "Sapienza" University of Rome, Policlinico Umberto I, \\ Department of Thoracic Surgery
}

Background: Prolonged air leakage is a common complication after pulmonary resections and it has been reported in $15 \%$ to $30 \%$ of cases. Prolonged air-leak is often associated with an increase of morbidity, hospital stays and costs. Several methods have been proposed to prevent and to treat air leakage. Autologous platelet leucocyte-enriched gel (PLG) is successfully used for the stimulation of tissue healing. Based on these features, PLG has been used to treat post-operative pulmonary air-leaks in 12 patients. PLG has been harvested by peripheral blood of patients with a relatively simple and low cost technique which drawn selectively platelets, growth factors and leucocytes with an antimicrobial function.

Materials and Methods: We have treated 12 patients with persistent air-leaks after pulmonary resection (7 lobectomies, 5 wedge resections). PLG is applied in the pleural space through chest tube; multiple doses may be injectioned. For each application we used about $80 \mathrm{ml}$ of gel. PLG can be used also for treating infection of residual pleural space and in case of incomplete reexpansion of lung parenchyma. Usually the first gel application is performed 10 days after surgery. In 2 patients PLG was performed in a single dose, in 7 patients two applications and in 3 patients three applications have been necessary.

Results: In all patients pulmonary air-leaks have been resolved after 48 hours by the last application of PLG. All patients have been discharged without complications.

Conclusions: In our experience autologous platelet leucocyte-enriched gel is used with efficacy to treat post-operative persistent air-leaks and infection of residual pleural space. 


\section{3}

\section{Unusual Methastatic Spread from Resected Masaoka IVa Thymoma}

\author{
N. Cassanelli, F. Davoli, G. Luciano, G. Dolci, \\ A.B. e Franco Stella \\ Università degli Studi di Bologna, Chirurgia Toracica. \\ Policlinico Sant'Orsola-Malpighi Chirurgia Toracica
}

Introduction: Thymoma is a rare tumour arising from anterior mediastinum. Surgery for early stages and multimodal management for the advanced ones can provide a durable remission of disease. Even many years after the first presentation, Thymomas can show metastatic spread. CASE Ten years ago a 55-years old woman came to our observation for a large mediastinal mass associated with multiple pleural lesions. Diagnosis of Thymoma was achieved by a transthoracic biopsy. After induction chemotherapy with remarkable debulking, we performed an extended thymectomy combined with a left pleuro-pneumonectomy. Masaoka staging was IVa. All the resection margins were negative. Follow-up was negative for 5 years until an abdominal CT-Scan revealed a splenic mass of about $3 \mathrm{~cm}$ : splenectomy was then performed. Two years later, a PET-Scan found a bone metastasis in a lumbar vertebra; after radiotherapy, a CT-PET Scan showed a left paracholic hypermetabolic spot, surgically removed by omental partial resection. To date the patient is disease-free .

Discussion: In advanced staged Thymomas, recurrences may occur in up to $50 \%$ of patients. Recurrence, if operable, should be surgical eradicated, offering good long-term survival. The exceptional nature of our case lies in the long disease-free period, in the site of the metastases and in the final outcome which was beyond all expectation.

\section{4}

\section{Long-term Results of Re-Resection for Recurrent Thymomas}

\section{S. Margaritora', G. Cusumano ${ }^{1}$, M. Chiappetta', F. Lococo', S. Cafarotti ${ }^{1}$, M. Vita ${ }^{1}$, V. Porziella ${ }^{1}$, E. Meacci', A. Evoli², S.P. Granone ${ }^{1}$ \\ ${ }^{1}$ Department of General Thoracic Surgery, Catholic University of Sacred Heart, Rome, Italy, ${ }^{2}$ Department of Neurology, Catholic University of Sacred Heart, Rome, Italy}

Objective: The treatment of recurrent thymomas remains controversial. The aim of this study was to analyze the early and long term results of surgical treatment for recurrent thymomas.

Methods: From 1972 to 2008, among 315 patients who underwent surgically complete resection for thymoma, 43 patients had a relapse of disease. Re-resection was attempted in 30 patients while the remaining patients were treated with radiotherapy/chemotherapy. Overall 5-year survival (LTS) and disease-free survival (DFS) outcomes were analyzed. Kaplan-Meier curves (Log-Rank) and Cox analysis were performed.
Results: Mean age and Female/Male ratio were 47.9 \pm 11.1 year and 21/22, respectively. Forty patients (93.0\%) had Myasthenia Gravis. A single relapse lesion was detected in 15 cases. The relapse sites were: pleura (\#28), mediastinum (\#16), lung (\#14), liver (1\#), and bone (\#1). A complete resection was achieved in $22 / 30$ cases (73.3\%). Peri-operative mortality and morbidity were $1 \%$ and $10 \%$, respectively. LTS and DFS rates after recurrence were $72.7 \%$ and $62.3 \%$, respectively. In the whole group, the patients who underwent surgery had better LTS compared with patients treated with radio and/or chemotherapy (LTS: $81.3 \%$ vs $53.8 \%$; $=0.015$ ) (HR:0.18;C.I.:0.18-0.5; $\mathrm{p}=0.001$ ). In the surgically treated group LTS was significantly better in case of complete re-resection $(93.3 \%$ vs $31.3 \%, \mathrm{p}=0.015)$ whereas the incomplete resection is associated with a poor prognosis (HR:6.12;C.I.:1.18-31.55; $\mathrm{p}=0.031$ ). No further differences in term of LTS and DFS where found according to the other considering clinical, surgical and pathological characteristics.

Conclusions: Surgical resection can be recommended in recurrent thymomas whenever possible. The completeness of reresection is the most important determinant of LTS.

\section{5 \\ Efficacy of Tigecycline Pleurodesis: A Comparative Experimental Study}

S. Ceccarelli, J. Vannucci, R. Capozzi, R. Pecoriello, V. Tassi, C. Maggio*, I. Bravi, M. Monacelli, D. Vinci, F. Marziani, A. Bufalari, N. Daddi.

Thoracic Surgery Unit in Perugia and Terni; "Department of Veterinary Pathology, Diagnostic and Clinic in Perugia; Institute of Pathologic Anatomy and Histology in Terni, University of Perugia. Italy

2nd Classified - SPIGC Award Session, Thoracic Surgery

Background: We investigated whether tigecycline (TIGE) is more effective than talc in inducing pleurodesis in rabbits.

Methods: 56 New Zealand rabbits were utilized in a two phase study: Effects at 14 days (PHASE I) and at 28 days (PHASE II) were assessed. Saline solution (SAL $n=3$ ), talc slurry (TALC $200 \mathrm{mg} / \mathrm{kg}, \mathrm{n}=5)$ and TIGE at different concentrations $(\mathrm{mg} /$ $\mathrm{kg})$ : TIGE0.5 $(\mathrm{n}=5)$; TIGE1 $(\mathrm{n}=5)$; TIGE3 $(\mathrm{n}=5)$; $\operatorname{TIGE} 25(\mathrm{n}=5)$; TIGE50 $(n=5)$ were randomly injected, for each phase, through a right chest drainage. TIGE0.5 and TIGE1 were ineffective during PHASE I and were thus excluded from further investigation.

At post-mortem examination, pleurodesis was graded grossly and microscopically by two observers, blinded to treatment groups.

Results: PHASE I: Pleurodesis was more effective in TIGE25 and TIGE50 $(\mathrm{p}<0.001)$;

TALC was better than TIGE0.5 $(\mathrm{p}<0.002)$ and TIGE1 $(\mathrm{p}<0.02)$, macroscopically. Inflammation, fibrosis and pleural thickness were significantly higher in TIGE25 and TIGE50 compared to SAL, TIGE0.5 and TIGE1 $(\mathrm{p}<0.02, \mathrm{p}<0.04, \mathrm{p}<0.05)$. No significant differences were evident between TALC and TIGE3, both macroscopically $(\mathrm{p}=0.11$ ), and microscopically (inflammation $\mathrm{p}=0.98$, 
fibrosis $\mathrm{p}=0.71$, pleural thickness $\mathrm{p}=0.48$ ). PHASE II: Better effectiveness of TIGE50 as compared to all other groups $(\mathrm{p}<0.05)$; results similar to PHASE I for TALC and TIGE3 ( $\mathrm{p}=0.57)$, macroscopically. Microscopically greater inflammation in TALC, compared to TIGE3 and TIGE25 $(\mathrm{p}<0.05)$. Significant complications occurred in all TIGE50 group. One of TIGE25 and one of TIGE50 died respectively for respiratory distress and for right hemothorax+ascites.

Conclusions: Intrapleural TIGE $3 \mathrm{mg} / \mathrm{Kg}$ is as effective as talc in inducing pleurodesis in rabbits. The intrapleural TIGE toxicity threshold was reached at TIGE25 $\mathrm{mg} / \mathrm{kg}$ concentration.

\section{6}

\section{Fluid Overload After Major Thoracic Surgery, A Well Recognized Hazard?}

\author{
V. Tassi, R. Capozzi, L. Cagini, R. Pecoriello, J. Vannucci, \\ S. Ceccarelli, C. Savignani*, G. Quintaliani*, M. Ragusa, \\ G. Angeletti, F. Puma
}

Thoracic Surgery Unit in Perugia and Terni, " Internal Medicine, Nephrology Unit, Internal Medicine, Section of Internal Medicine and Endocrine and Metabolic Sciences. University of Perugia. Italy

Objectives: Aim of this observational study is to evaluate the amount and the distribution of the Total Body Water (TBW) along with the variation of specific hormones and electrolytes in patients submitted to pulmonary lobectomy for lung cancer.

Patients and Methods: 34 consecutive patients were enrolled. The following data were analyzed before surgery and in postoperative (postop) day 1,3,5 and 7: body weight, adrenocorticotropic hormone (ACTH), serum and urinary cortisol, Atrial Natriuretic Peptide (ANP). Hydration was evaluated by hematocrit (Ht), blood urea and creatinine, plasma proteins, Sodium and Potassium value, urine osmolality. Monofrequency bio-impedance analysis (BIA) was utilized to quantify at the same time the water gain and redistribution of TBW.

Results: There was no mortality and major morbidity. Estimated mean intraoperative blood loss was $250 \mathrm{ml}$. No blood transfusions were required. The following results were observed in all patients, up to postop day 4: increase of body weight (0.6$13.8 \%$, range $0.500-9 \mathrm{~kg}$ ), increase of ANP and of cortical-adrenal function, slight decrease of hematocrit and plasma proteins, probably due to hemodilution as confirmed by urea and creatinine values. Urine osmolality did not modified in the observational period. The mean bio-impedance derived reactance and mean body weight are depicted in fig 1. Restoration of the preoperative values was observed between day 5 and 7 .

Conclusions: Our data show that postoperative gain weight and oedema formation, are related to the pronounced increase of corticoid-adrenal function in response to the surgical stress. Strict fluid restriction, diuretics and secondary secretion of ANP do not avoid such imbalance. Hemodilution seems to have a minor role. BIA is more sensitive then body weight to monitor fluid redistribution. Further experience is required.

\section{7 \\ Bronchovascular Sleeve Resection: An Effective Alternative Option to Pneumonectomy in NSCLC}
A.F. De Filippis, A. Zuin, M. Schiavon, G. Marulli, A. Rebusso, S. Nicotra, M. Loy, F. Rea

Background: Pneumonectomy may be associated with high rate of postoperative morbidity and mortality, therefore, every effort to avoid this procedure should be carefully considered.

In selected cases, bronchial sleeve resections, associated with angioplastic procedures, could represent a valid alternative to pneumonectomy in NSCLC.

Aim of this study is to compare the impact of these procedures in terms of short- and long-term results.

Methods: A retrospective study was carried out by reviewing records of 54 consecutive patients submitted to sleeve lobectomy and vascular reconstruction for NSCLC at our Institution from January 1990 to December 2008. On the basis of clinical, surgical and pathological characteristics a control group of 54 patients submitted to pneumonectomy was matched for comparison.

Results: Sleeve group included patients with a median age of 67 years; in 24 cases a sleeve resection of the pulmonary artery was associated. Pathologic staging revealed 5 stages Ib, 19 IIb, 23 IIIa, $5 \mathrm{IIIb}$ and $1 \mathrm{IV}$; in one case, after neoadjuvant chemotherapy, there was no evidence of residual disease.

In sleeve and pneumonectomy groups perioperative mortality rate was $1.85 \%$ and $7.4 \%$, respectively $(\mathrm{p}=0.16)$; morbidity rate was $16.7 \%$ in both groups. Overall 5 -year survival rate was $32 \%$ and $28 \%$, respectively $(\mathrm{p}=0.32)$.

Conclusion: Compared with pneumonectomy, sleeve resections with vascular reconstruction revealed a comparable morbidity, less mortality and better long-term survival.

Therefore, whenever feasible, parenchyma sparing surgery, associated with vascular procedures, should always be attempted.

\section{8}

\section{First Experience with Allograft Sternochondral Replacement}

F. Di Chiara*, G. Marulli, A. Zuin*, C. Breda*, M. Schiavon*, A. Rebusso*, A.F. De Filippis*, V. Scapin ${ }^{+}$, F. Rea*

*Department of Cardiothoracic and Vascular Sciences, Division of Thoracic Surgery, University of Padua,

+ Institute of Radiology, University of Padua

Surgical excision with a safety margin is a primary goal in the treatment of malignant sternal tumors. The aim, after sternal resection, is to prevent respiratory impairment due to flail chest and deformity. Various techniques and materials have been proposed for this intervention. This report describes the use for the first time of sternal allograft to reconstruct the chest wall in three patients. 
Two males and one female, with age of 48, 51 and 52 presented with neoplastic lesions of the sternum. One chondrosarcoma, one metastasis from breast cancer and a sternal metastasis from hepatocarcinoma in a patient patient with a previous liver transplantation for HCC in HCV-related cirrhosis. In all cases the tumors presented as osteolityc lesion with involvement of the costal cartilages and the surrounding soft tissues for the breast cancer metastasis. The dissection was performed via elliptical incision preserving, when possible, the pectoralis majors. In two cases the sternal resection was incomplete, preserving part of the manubrium, in only one case a complete sternectomy was carried out. The defect was reconstructed by using an allograft sternum including costal cartilages that was previously treated with antibiotic solution and cryopreserved at $-80{ }^{\circ} \mathrm{C}$. This process guarantees the sterility of the graft and the absence of immunogenic capacity. The graft was tailored to perfectly fit to the defect and fixed with titanium plates and screws. Postoperative course was uneventful. One patient required removal of one plate and screw, four months after the operation, because they dislocate, though the graft is well positioned.

\section{9}

\section{Analysis of Prognostic Factors in Surgically Resected N1 Non-Small Cell Lung Cancer}

\section{E. Di Prima, L. lacuzio, C. Casali, A. Stefani, U. Morandi \\ Cattedra e Struttura Complessa di Chirurgia Toracica, Università di Modena e Reggio Emilia}

Introduction: Patients with N1 non-small-cell lung cancer represent an heterogeneous group, with different prognosis. Surgical resection is the treatment of choice the type of resection represents a subject of debate: lobectomy is traditionally considered the gold standard but possible better survival after pneumonectomy has been suggested.

Materials and Methods: Retrospective study of T1-T3N1 NSCLC undergoing complete major pulmonary resection, between 1990-2009. The prognostic role of N1 descriptors, according to the current and the new $7^{\text {th }}$ edition of the TNM staging system was investigated, as well as the impact on survival of the type of resection.

Results: 384 patients were analyzed. 5-year survival was $46 \%$. Involvement of hilar station, multiple stations and multiple nodes resulted as poor prognostic factors (5-ys of 33\%, 21\% and $30 \%$ respectively), as well as involvement of hilar zone and multiple zones (5-ys of $27 \%$ and $23 \%$ respectively). Pneumonectomy showed better survival rates with respect to lobectomy/bilobectomy ( $58 \%$ vs. $42 \%, p=0.058$ ); when considering hilar zone, this difference became significant ( $60 \%$ vs. $29 \%$ respectively, $p=0.030$ ). Postoperative mortality was comparable. The type of resection remained independent prognostic factors at multivariate analysis.

Conclusion: Our study confirmed the different prognosis of N1 NSCLC. Patients with hilar nodes or multiple levels or zones involvement showed poor prognosis. Pneumonectomy was significantly associated to improved survival, likely because it provided a more accurate clearance of the lymphatic drain system with respect to lobectomy, especially when hilar and interlobar nodes were involved. Further studies on tailored adjuvant therapy for different N1 subgroups and on the role of pneumonectomy are warranted.

\section{0}

\section{Early Stage NSCLC in High Risk Patients: Wedge Resection vs Radiofrequency Ablation}

O. Fanucchi, M.C. Ambrogi, P. Dini, A. Mussi

Chirurgia Toracica, Dipartimento Cardiotoracico, Università di Pisa

Introduction: Surgeons are frequently faced with patients with early stage NSCLC at high risk for major pulmonary resection due to their poor clinical status. So limited resections, such as wedge resection, or non-surgical therapies, like radiofrequency ablation (RFA), can be considered as alternative approaches.

Materials and Methods: We reviewed patients with early stage NSCLC, that underwent wedge resection or RFA during the period 2006-2009. We analysed and compared mortality and morbidity rate, local and distant recurrence rates, actuarial and cancer-related survival.

Results: 32 patients ( 4 females and 28 males, mean age 70 years) underwent wedge resection. 34 patients ( 7 females and 27 males, mean age 74 year) were treated with RFA. The mean diameter of lesions was $2.3 \mathrm{~cm}$ for wedge resection and $2.6 \mathrm{~cm}$ for RFA. There was no mortality in both groups, morbidity rates were $31.5 \%$ and $26.5 \%$ respectively. At a mean follow-up of 29.9 months for wedge resections and 31.6 months for RFA, the 1-, 3-, 5-year actuarial survival rates were respectively 100\%, 67\%, 50\%, and $94 \%, 54 \%, 41 \%$ for RFA, with no statistically significant difference. Disease free rates were respectively $78 \%$ and $56 \%$. Higher local recurrence rate was observed for RFA $(41.1 \%)$ respect to wedge resections $(6.2 \%)(\mathrm{p}=0.002)$.

Conclusion: Wedge resection and RFA appear to be safe and viable compromises for treatment of eraly stage NSCLC, in patients with inadequate pulmonary reserve or high co-morbidities. RFA is associated with an increased risk of local recurrence, so it should be reserved for patients with absolute contraindication to surgery. 


\section{1}

\section{Penetrating Atherosclerotic Ulcer of the Ascending Aorta Two Years After a Lung Lobectomy}

\author{
N. Filippi', A. Cappai 2, M. Montoli', E. Fiorini' \\ ${ }^{1}$ Department of Thoracic Surgery, University of Milan, \\ Fondazione Osp. Maggiore Policlinico, IRCCS, Milan, Italy, \\ 2Department of Cardiovascular Sciences, University of \\ Milan, Centro Cardiologico Monzino, IRCCS, Milan, Italy
}

Introduction: A 68-year-old male, with previous left upper lung lobectomy for cancer and abdominal aortic aneurism repair, was submitted to control contrast multi-slice computed tomography (CT), which showed ascending aorta dilatation $(53 \times 48 \mathrm{~mm})$ with a penetrating ulcer, $9 \mathrm{~mm}$ in depth and $20 \mathrm{~mm}$ in diameter.

Materials and Methods: Preoperative aortic and coronary angiography revealed a right coronary artery (RCA) critical stenosis, but the presence of the ulcerative lesion was not detected. The lesion was confirmed in surgery and a pathological specimen was collected. The patient underwent successful ascending aorta replacement with a Dacron tube and myocardial revascularization on RCA with a saphenous vein graft.

Results: Asymptomatic penetrating atherosclerotic ulcer is a rare pathology of the ascending aorta, more frequent in the descending thoracic aorta, even in multiple localizations. CT scan and magnetic resonance imaging are the best preoperative methods to diagnose ascending aorta ulcer, a pathological entity with high risk of rupture or progression in intramural hematoma and acute dissection.

Conclusion: The management of this uncommon case needs multidisciplinary approach, first of all thoracic and cardiovascular surgeons together with radiologist.

\section{2}

\section{Diagnosis of the Anterior Mediastinal Tumor: Is 99m Tc-MIBI-SPECT a Reliable Diagnostic Tool?}

\section{A. Fiorelli, G. Vicidomini, G. Messina, P. Rambaldi, L. Mansi, A. Rotondo, M. Santini}

Thoracic Surgery Unit, Second University of Naples

Objective: The role of ${ }^{99 \mathrm{~m} T c-M I B I-S P E C T}$ in the detection of anterior mediastinal mass.

Methods: 30 consecutive patients with an isolated anterior mediastinal mass at Computed Tomography were enrolled. All patients underwent ${ }^{99 \mathrm{~m}} \mathrm{Tc}-\mathrm{MIBI}-\mathrm{SPECT}$ before invasive diagnostic procedures and/or surgical resection. The uptake of the mediastinal tumor $(\mathrm{T})$ was compared to the normal tissue $(\mathrm{N})$ and $\mathrm{T} / \mathrm{N}$ ratio was calculated to define the metabolic activity of the lesions.

Patients were divided in six groups according histologic patterns which were then correlated to $\mathrm{T} / \mathrm{N}$ value: benign tumor (BT), lymphoma (LYM), other malignant tumor (OMT) and lowrisk thymoma (LRT) including types $\mathrm{A}, \mathrm{AB}$ and $\mathrm{B} 1$, high-risk thy- moma (HRT) types B2 and B3, and Thymic Carcinoma according a simplified WHO histological classification. Yet for thymoma, the size, Masaoka stage and miastenia were valuated.

Results: There were: 5 BT, 8 LRT ( $4=\mathrm{A}, 2=\mathrm{AB}, 2=\mathrm{B} 1)$, 4 HRT ( $3=\mathrm{B} 2,1=\mathrm{B} 3$ ), 4 Thymic Carcinoma, 6 LYM and 3 OMT. The T/N of BT $(1.1 \pm 0.1)$ was significantly lower than that of HRT $(2.6 \pm 0.1$, $\mathrm{p}=0.01)$, of Thymic Carcinoma $(2.9 \pm 0.1, \mathrm{p}=0.0001)$, of LYM $(2.8 \pm 0.1, \mathrm{p}=0.0001)$, and of OMT $(2.9 \pm 0.2 ; \mathrm{p}=0.0001)$ while there is no significant difference between BT and LRT ( $\mathrm{p}=0.06$ )(Figure 1). Regarding thymoma, the differences between the histologic subgroups (LRT vs HRT: $\mathrm{p}=0.004$, HRT vs Thymic Carcinoma: $\mathrm{p}=0.02)$ and Masaoka stage $(\mathrm{r}=0.9, \mathrm{p}=0.0003$, Spearman's test $)$ were statistically significant while the size of the lesion $(\mathrm{r}=0.4, \mathrm{p}=0.1)$ and myasthenia $(\mathrm{p}=0.9)$ were no significant factors.

Conclusion: ${ }^{99} \mathrm{~m}$ Tc-MIBI-SPECT is a reliable diagnostic tool in distinguishing benign from malignant malignant anterior mediastinal mass and in predicting histologic types of thymoma.

\section{3 \\ Diagnosis of Pleural Fluid and the Role of Vascular Endothelial Grow Factor (VEGF)}

A. Fiorelli, G. Vicidomini, P. Laperuta, G. Messina,
F. Morgillo, F. Ciardiello, M. Santini

Thoracic Surgery Unit, Second University of Naples

Objective: To evaluate the value of VEGF in differential diagnosis of pleural effusion (PE)

Methods: 79 consecutive patients presenting with undiagnosed unilateral PE were enrolled. PEs were categorized as transudates or exudates including benign and malignant effusions. Pleurosies were then splitted into benign and malignant according pathologic findings. In all patients, VEGF levels $(\mathrm{pg} / \mathrm{mL})$ were measured using ELISA and then correlated to aetiology of PEs.

Results: There were 14 transudative PEs and 65 exudative PEs including 49 malignant PEs and 16 benign PEs. VEGF levels in transudative were significantly lower than those in exudative PEs $(272 \pm 118$ vs $746 \pm 435, \mathrm{p}<0.0001)$. Among exudative PEs, VEGF concentrations were significantly higher in malignant effusions than those of benign effusions ( $778 \pm 404$ vs $381 \pm 218, \mathrm{p}<0.0001)$. No difference was observed between the different sub-groups of malignant PEs split according to histology or primary tumor localization. Cut-off point of VEGF levels for malignant PEs was 386 with sensitivity and specificity of $91 \%$ and $83 \%$, respectively (ROC curve). However 22/23 patients with cytology-negative malignant PEs could be identified by VEGF.

Conclusion: VEGF levels are significantly raised in malignant compared with benign PEs and its elevation may indicate the need for confirmatory pleural biopsy. 


\section{4 \\ A Rare Case of Sclerosing Hemangioma of the Lung \\ M. Gallazzi, L. Luzzi, L. Voltolini, C. Ghiribelli, F. Granato, \\ S. Tenconi, G. Gotti \\ U.O.C. Chirurgia Toracica A.O.U. Senese}

Introduction: We present a case of Sclerosing Hemangioma $(\mathrm{SH})$, a rare benign neoplasm of the lung. This kind of tumours originate from the pulmonary epithelial cells, generally it is characterized by a slow-growing in middle-aged females.

Materials and Methods: During the preoperative work-up for an uterine leiomioma, a 43 years old female in good clinical conditions, undergoes Chest X-Ray that revealed a coin lesion in the left lower lobe (LLL). The CT scan showed an enhancing, subpleural mass of $35 \mathrm{~mm}$ with calcified areas, corresponding to the apical segment of the LLL. Four mounts later the follow up CT scan showed an increased size of the lesion. Indication for surgical resection was set. The intra-operative biopsy resulted positive for NSCLC, than a left inferior lobectomy was performed. The specimen analysis showed a well-circumscribed tumour, histologically composed by an heterogeneous cellular types, compatible with a $\mathrm{SH}$. The postoperative was regular and the patient was discharged after 7 days.

Results: Benign tumours are around $4 \%$ of all neoplasm of the lung. The $\mathrm{SH}$ is infrequent and predominantly affects middleaged women. Most tumours are solitary, peripheral and wellcircumscribed; a spread to regional lymph nodes is reported in approximately $1 \%$ of cases. Despite asymptomatic in $70-80 \%$ of cases, they can be associated with caught and haemoptysis, if of great dimensions.

Conclusions: $\mathrm{SH}$ must be considered in the differential diagnosis of pulmonary lesions with benign caracteristique.

\section{5}

\section{Atypical Carcinoid Tumor of the Lung: Our Last-10-years Experience}

\author{
S. Giarratana, F. Davini, M. Lucchi, M.C. Ambrogi, A. Mussi \\ Division of Thoracic Surgery, Cardiac and Thoracic \\ Department, University of Pisa, Pisa
}

Introduction: Carcinoid tumours of the lung are rare, representing 1-3\% of all lung tumours. They are distinguished in typical (TC) and atypical (AC), with the latter associated with a poor prognosis. We review our experience with bronchopulmonary AC in the last 10 years.

Materials and Methods: From our surgical database we picked up those patients treated for bronchopulmonary carcinoid tumour and among these ones those with AC. We collected data from general registry, pre-operative diagnosis and staging, surgical treatment, pathological diagnosis and staging (according to WHO-2004 and UICC-2009 respectively), follow-up.
Results: From 2000 to 2009, 116 patients underwent surgical treatment for bronchopulmonary carcinoid at our Department. In 26 cases the pathological diagnosis was AC. They were 6 males and 20 females with a mean age of 56 years (range of 25 - 82). We performed a pneumonectomy in 2 cases, a lobectomy in 19 and a wedge resection in 6 . Mediastinal lymphadenectomy was always done. Pathological staging was: $1 \mathrm{~A} \mathrm{n}=10,1 \mathrm{~B} \mathrm{n}=6,2 \mathrm{~A} \mathrm{n}=5,2 \mathrm{~B}$ $\mathrm{n}=3,3 \mathrm{~A} \mathrm{n}=1,3 \mathrm{~B} \mathrm{n}=1$. We observed lymph node metastases in 7 patients ( $5 \mathrm{~N} 1$ and $2 \mathrm{~N} 2$ ). At a mean follow-up of 64 months median overall survival was 57 months, while actuarial survival at 5 and 10 -years were $88 \%$ and $75 \%$.

Conclusion: Management of AC should be the same as for NSCLC, above all the surgical procedure. In this case overall survival is not so dissimilar of TC.

\section{6}

\section{Hypoglycemia, Lymphedema and Cardiac Arrhytmia in Patients Over 80 Years Old with Giant Intra-thoracic Solitary Fibrous Tumor Brief Reports}

\author{
F. Givigliano', S. Fechner ${ }^{1}$, M. Töetsch², G. Stamatis ${ }^{1}$ \\ ${ }^{1}$ Department of Thoracic Surgery and Endoscopy, \\ Ruhrlandklinik/University Essen, Germany, ${ }^{2}$ Institute of \\ Pathology and Neuropathology, University of Essen \\ Germany
}

Introduction: The Solitary Fibrous Tumor of the pleura (SFTPs) is a rare spindle-cell mesenchymal tumor originated from the mesenchymal tissue and is the most common benign tumor of the pleura. The surgical removal represents the standard treatment. Surgical treatment in old patients is always discussed, but the recent literature stresses that the surgical approach depends not by the age but by the patient's general conditions.

Methods: Three patients over 80 Years old with a Giant SFTPs were uncomplicated surgically treated with the removal of the Giant tumor. All the patients were referred to our department after the radiological evidence of a big intra thoracic neo-formation during investigations carried out to clarify the occurrence of a generalized symptoms such dyspnoea, arrhythmia, lymphatic disorder of the legs and severe hypoglycemic syndrome with syncopal episodes.

Result: The surgically treatment was uneventful in all the cases and the patients were discharged with complete remission of the related symptoms.

Conclusion: We have evidenced that even in very old patient the surgical removal of SFTPs is acceptable as Gold Standard treatment and can be uneventful. A carefully pre and post operative management is necessary but the contraindication for the surgery is not the age, but the general conditions of the patients. 


\section{7}

\section{Combined Thoracic and Neuro-surgical Approach for an Unusual Intrathoracic and Neck Neurofibromatosis. Case Report}

\section{F. Givigliano, G.F. Menconi}

\section{Sez. Chir Toracica Ospedale Livorno}

Introduction: Sometimes the evidence of lesions in border anatomic districts, needs interaction between different specialists, in order to obtain a complete and safety resection.

Materials and Methods: A 25-years-old man affected by multiple neurofibromatosis, with a past of surgical removal of similar lesions in many body districts showed in the CT scan the evidence of expansive lesion of $9 \mathrm{~cm}$ from the left part of the $3^{\text {th }}$ cervical vertebra, up to the left pulmonary artery. The patient was tachycardic too. With the cooperation of the Neurosurgeon, to treat any possible cohesion with the brachial nervous plexus, we performed during the same surgical time a contemporary left cervicotomy and a left anterior minitoracothomy, and we have found two different capsulated lesions, adherent not infiltrated carotid, brachial nervous plexus and the lung apex with a compression of the left pulmonary artery. The lesions were removed part from the thorax and part from the neck.

Result: Final pathologic examination was: "Capsulated neurofibroma partially ialin with atypicalls cells and free margins". The tachycardic syndrome disappeared after the surgical procedure, and the Chest X-ray showed a complete lung re-expansion.

Conclusion: Multi approach treatment of lesions within the supra-clavicula fossa with thoracic invasion is well described in literature and we underline the importance of using different specialists to approach a complex lesion in a middle district, in order to treat safety the different structures.

\section{8 \\ Cortical Thymic Epithelial Tumors Have an Increased Risk of Developing Additional Malignancies: Lack of Immunologic Surveillance? \\ F. Granato*, D Spina**, S. Lazzi**, L. Voltolini*, M.R. Ambrosio**, L. Luzzi*, S. Bongiolatti*, G. Gotti, L. Leoncini** \\ ${ }^{*}$ Thoracic Surgery Unit, Department of Cardiothoracic and Vascular Surgery, Siena University Hospital, Siena, Italy, \\ **Anatomic Pathology Section, Department of Human Pathology and Oncology, Siena University Hospital, Siena, Italy}

Background: The increased risk of developing an additional malignancy after a thymic neoplasm has not yet been fully examined. Moreover, previous studies have found the histologic pattern of thymic tumors to be unrelated to the growth of additional tumors.
Methods: The Authors studied 52 patients who underwent surgical excision of a thymic epithelial tumor (TET group). Based on the WHO classification, two sub-groups were formed: subgroup 1 (medullary tumors, 29 patients affected by A, AB1 and B1 thymomas) and sub-group 2 (cortical tumors, 23 patients affected by $\mathrm{AB} 2, \mathrm{~B} 2, \mathrm{~B} 3$ thymomas and thymic carcinomas). A control population was provided by the creation of a further database comprising 114 patients with diagnosis of colorectal cancer was created (CC group). RESULTS Patients in the TET group showed a statistically significant higher risk of developing additional malignancies compared to patients in the CC group (12/52 vs $11 / 114$ patients, $\mathrm{p}=0.0374)$. The association between TETs and additional malignancies was related to the TET histotype; sub-group 2 (cortical tumors) showing a statistically significant higher risk of developing an additional malignancy $(\mathrm{p}=0.008)$. The time interval (TI) between the appearance of the first and second tumor in the TET group was significantly shorter than the TI in CC group $(\mathrm{p}=0.014)$, with TETs anticipating the growth of additional tumors in many cases $(\mathrm{n}=10)$.

Conclusion: Patients affected by TETs have a significantly higher risk of developing additional tumors and this risk is considerably greater in tumors arising from the thymic cortex. This result may be due to the more aggressive biological features of cortical TETs (cTETs) and suggests a primary role for cortical thymic epithelial cells in immunologic surveillance and defence against neoplasms. The generally low TI values between TETs and other malignancies support the potential presence of an immunological impairment that often appears prior to evidence of TET.

\section{9}

\section{Evalutation of Pleuraseal ${ }^{\mathrm{TM}}$ to Reduce Air Leaks in Lung Resection on Re Thoracotomy}

\author{
P. Maniscalco, W. Grossi, F. Quarantotto, C. Bottoli, \\ S. Perna, E. Garelli, G. Cavallesco \\ Division of General and Thoracic Surgery, Azienda \\ Ospedaliero Universitaria S.Anna, Ferrara
}

Introduction: Air leaks after pulmonary resection may contribuite to increased patient morbidity, delayed removal of chest drainages tubes and prolonged hospitalization.

We report our experience on efficacy of the pleuraseal lung sealant to stop pulmonary air leak after pulmonary resection on redothoracotomy.

Patients and Methods: There were 6 patients submitted to redo thoracotomy for malignancy lung disease with an evidence intraoperative air leak treated with Pleurasel Hydrogel. In 4 patients was performed a sub-lobar resection while the other two cases received a lobectomy. Intraoperative leakage was graded according to Macchiarini's scale and restaged after Hydrogel application. Postoperative air leaks were catalogued twice daily according to the classification reported by Cerfolio and coll.

Results: Four patients received a lung sealant application for grade 2 air leak. One had an evidence of grade 3 air leak and was treated with more applications of sealant. The last one with grade 4 air leak underwent further standard techniques to reduce all leaks until to grade 2 and after pleuraseal can be applicated. 
Repeat submersion testing was negative (grade 0 ) in three patients and the other three subject have a grade 1 air leak. On postoperative day one, air leak was detected in five patients. In all patients the air leaked stoped within 3 days. The chest tube was removed after mean 4.8 days. One patients had a pneumothorax and readmitted two days after discharge.

Conclusion: The use of pleuraseal device after pulmonary resection on redo thoractomy may be helpful on the treatment of alveolar air leak.

\section{0 \\ VATS Decortication for Management of Pleural Empyema: A Single Centre Experience \\ P. Maniscalco, F. Quarantotto, C. Bottoli, S. Perna, W. Grossi, E. Garelli, G. Cavallesco \\ Division of Thoracic Surgery, Sant'Anna Hospital University of Ferrara}

Introduction: Pleural empyema remains a frequently encountered clinical problem and is responsible for significant morbidity and mortality worldwide. Delays in diagnosis and treatment may contribute to morbidity, complications, and mortality. The management of parapneumonic empyema depends on timely, stage-dependent therapy and the underlying etiology. Our purpose in this study was to assess the safety, feasibility and efficacy of the video-assisted thoracoscopic surgery (VATS).

Materials and Methods: From January 2002, to December 2008, VATS debridement and decortication in 49 consecutive patients presenting with pleural space infections was performed with endoscopic approach. A retrospective review was performed and the effect of this technique on perioperative outcome was assessed.

Discussion: Fortynine patients were 27 male, 22 females and the mean age was $59.4 \pm 13.4$ years. Fifteen patients underwent chest tube before VATS or thoracotomy because of their conditions. The VATS evacuation of infected pleural fluid and decortication was successfully performed in 35 of 49 patients. VATS is a safety and efficacy procedure in the treatment of early stage empyema. Use of VATS in early period of multiloculary empyema, prevents patients from unnecessary thoracotomies.
251

\section{Use of Thoracic Muscular Flap in the Management of Tracheo-Bronchial Fistulae}

\section{Libretti, A.V. Bedini}

Department of Emergency-Urgency, General Surgery and Transplantation, Obesity Surgery Center, University of Bologna, "Department of Emergency-Urgency, General Surgery and Transplantation, surgical endoscopy unit

Introduction: The treatment of tracheo-bronchial and broncho-pleural fistulae remain a major challenge for the thoracic surgeon. We present a series of five consecutive patients who underwent closure of the fistula with a muscular flap.

Materials and Methods: From June 2009 to April 2010 five patients were diagnosed with tracheal or bronchial fistula. Three patients had dehiscence of the bronchial stump after pneumonectomy. One patient presented with a fistulization of the gatric tube in both bronchi after a total esophagectomy. The last patient had a tracheal rupture after intubation.

Results: The three patients with dehiscence of the bronchial stump were treated with a pectoralis major flap associated to an open thoracic window thoracostomy. The defect was not closed directly but the muscle was inserted into the open fistula and sutured to its edge. In only one patient the pectoralis major muscular flap failed; he was then treated successfully with an omentum flap.

In the other two patients the defect was sutured directly and then covered with a latissimus dorsi flap.

All patients are alive and well (survival from the intervention $7,1 \pm 4$ months).

Conclusions: Muscle flaps represent an excellent opportunity to treat successfully broncho-pleural or tracheal fistulae. An omentum flap though can be successfully used when a muscular flap fails.

\section{2}

A Pulmonary Lobectomy Entirely Realized with Ligasure ${ }^{\mathrm{TM}}$ Vessel Sealing System: Is It a Reasonable Alternative to Conventional Ligatures?

\section{G. Luciano, F. Davoli, N. Cassanelli, G. Dolci,} A.B. e Franco Stella

Università degli studi di Bologna, U.O.Chirurgia toracica, Policlinico S.Orsola Malpighi

Introduction: Many recently published studies describe the efficacy and the safety of LigaSure ${ }^{\mathrm{T} m}$ Vessel Sealing System in thoracic surgery for pulmonary atypical resections or fissure separations in human and for pulmonary major vessel division in experimental animal models.

Materials and Methods: We report a case of a pulmonary lobectomy successfully performed on a 55 years-old cau- 
casic woman with NSCLC using a bipolar thermal vessel sealer (LigaSure $^{\mathrm{Tm}}$ Vessel Sealing System).

Results: We realized an upper right lobectomy, by a posterior-lateral thoracotomic approach, using LigaSure ${ }^{\mathrm{mm}}$ Vessel Sealing System to: enter the pleura space, seal segmental branches of the right pulmonary artery and of the right superior pulmonary vein, divide incomplete interlobar fissures, incise the mediastinal pleura in order to achieve systematic limphadenectomy and for haemostasis. Operative time was of 120 minutes; we couldn't observe any intra-operative bleeding; post-operative course was uneventful without prolonged air leaks. One year follow-up reveals no procedure-related complications and absence of neoplasm relapse.

Conclusion: In our opinion, LigaSure ${ }^{\mathrm{TM}}$ Vessel Sealing System could actually find application in anatomical resections for NSCLC early stages. In the future we hope to achieve larger series of patients in order to gain more data about this useful device.

\section{3 \\ Long Term Results of the Heller-Dor Operation with Intraoperative Manometry for the Treatment of Esophageal Achalasia

\begin{abstract}
M. Mandrioli' ${ }^{1}$ S. Mattioli ${ }^{1}$, A. Ruffato ${ }^{1}$, V. Pilotti', B. Aramini', F. D'Ovidio ${ }^{2}$

1Division of Thoracic Surgery, Villa Maria Cecilia Hospital, Cotignola (Ravenna), Center for the Study and Therapy of Diseases of the Esophagus. Alma Mater Studiorum University of Bologna, Italy, ${ }^{2}$ Division of Cardio-Thoracic Surgery, Columbia University, New York, U.S.
\end{abstract}

Objective: The details of Heller myotomy and Dor fundusplication for the cure of dysphagia (D) and the occurrence of reflux esophagitis (RE) were analyzed in a 30 years experience.

Methods: From 1979 to 2008202 patients (97 men, median age 55.5 yrs. r. 7-94) were operated on by laparotomy (L) and 60 (24 men, median age 56 yrs. r. 16-80) by laparoscopy (LX). Intraoperative manometry was used to: 1) abolish the high pressure zone (HPZ) with a long esophago-gastric myotomy 2) protect the surface of the myotomy with a long but soft anterior fundusplication (trimmed to avoid RE without impairing the esophageal emptying). A semiquantitative scale graded results.

Results: Mortality was $1 / 202$ in the L and $0 / 60$ in the LX with 2 conversions. FU: L median 96 months r. 12-324, LX median 48 months (r. 6-161). Myotomy achieved the complete abolition of $\mathrm{HPZ}$ in $100 \%$; the Dor related HPZ mean length and pressure were $4.5( \pm 0.4) \mathrm{cm}$ and $13.3( \pm 2.2) \mathrm{mmHg}$ in $\mathrm{L}$ and $4.5( \pm 0.5) \mathrm{cm}$ and $13.2( \pm 2.2) \mathrm{mmHg}$ in LX $(\mathrm{p}=0.75)$. In L poor results $(19 / 201$ $9.5 \%$ ) were secondary to RE in $15 / 201(7.5 \%)$, in $2 \mathrm{RE}$ was diagnosed after 184 and 252 months and to recurrent D in 4/201 (2\%) all with end stage sigmoid achalasia. In LX 2/60 (3.3\%) had RE and none recurrent $\mathrm{D}$.

Conclusions: A long esophago-gastric myotomy and Dor fundusplication performed under manometric parameters is probably the key factor for the optimal cure of D and efficiently controls postoperative RE.

\section{4}

Pleuro-Pulmonary Complications in a Patient Affected by Rheumatoid Arthritis: A Real Challenge for Thoracic Surgeon. A Case Report

S. Sanna, D. Argnani, M. Monteverde, M. Taurchini, M. Mengozzi, D. Dell'Amore

U.O. Chirurgia Toracica - Ospedale G.B. Morgagni - Forlì

Only $1 \%$ of patients affected by rheumatoid arthritis suffer of pleuro-pulmonary complications, as pneumothorax and pleuroparenchimal fistula. A correct treatment is difficult because of chronic inflammation and immunodepressant therapy taken by the patient, undergone to repeated surgery and to a long, difficult course, often unsuccessful.

Case Report: A young woman affected by rheumatoid arthritis in immunodepressant and cortisonic therapy presented fever and right hidropneumothorax. A chest CT shows bilateral pleural effusion and multiple pleuro-pulmonary nodules. A left pneumothorax occurred, and she underwent right videothoracoscopic decortication with pleural biopsies revealing the rheumatoid origin of nodules. Due to bilateral persistent air leakage, resistant to all types of pleurodesis, we performed a left VATS decortication, without any result. The patient was discharged to the hospital with bilateral thoracic drainage and later underwent right pleurectomy, without any result. Actually she has permanent bilateral chest drainage.

Conclusion: Patients affected by pleuro-pulmonary complications of rheumatoid arthritis represent a difficult therapeutic challenge, due to failure of common techniques for VATS pleurodesis. Few reports propose an early surgical approach in these cases to prevent pleural adherences and chronic rheumatoid illness, major causes of treatment failure.

\section{5 \\ Broncho-Pleural Fistula After Lung Surgery: New Trends in Treatment. Our Experience}

S. Sanna, D. Argnani, M. Monteverde, M. Taurchini, M. Mengozzi, D. Dell'Amore

U.O. Chirurgia Toracica - Ospedale G.B. Morgagni - Forlì

Broncho-pleural fistula with pleural empyema represent a frightful surgical complication, with high mortality and morbidity. The best correction of the fistula is still debated. In early fistula direct suture is indicated, in late fistula pleural empyema must be treated before. Small fistulae are treated endoscopically with synthetic glue, large fistulae need surgical treatment and many are the available techniques.

Cases Report: A patient treated with pleuro-pneumonectomy presented a small broncho-pleural fistola $<2 \mathrm{~mm}$. treated with a combined closure, injecting the fistula with synthetic glue endoscopically and treating the empyema videothoracoscopically. A patient treated with right lower lobectomy developed a bron- 
cho-pleural fistula and empyema treated with surgical closure and omental flap trasposition. A large post-pneumonectomy bronchopleural fistula with empyema was treated first with "open window" thoracostomy then with suture and omental and muscolar flap transposition to fill in the cavity.

Conclusion: The techniques for broncho-pleural fistula correction are various, from endoscopic closure to combined treatment endoscopic/VATS until suture and omental or muscolar flap trasposition. Multiple are the variables to consider for a better strategy: dimension, time of arising, patient's conditions and site, to obtain the best result with minimal patient's discomfort and risk.

\section{6}

Neuroendocrine Tumors of the Lung: Results of Surgical Treatment in a Series of 147 Patients

D. Angela, L. Domenico*, A.N. Pia, P. Vincenzo,

S. Francesco*, L. Michele

Chirurgia Toracica

Introduction: Neuroendocrine tumors (NET) of the lung represent about $22 \%$ of all lung neoplasms. We evaluated prognostic factors and long-term survival in patients surgically treated for lung NET at our Institutions.

Methods: From January 1987 to December 2009, 147 patients (88 men, 59 women), mean age 57 years (range: 16-78 years), underwent lung resection for NET. All cases were rewieved according to the WHO/IASLC classification (1999) and divided in tumorlets, typical carcinoid (TC), atypical carcinoid (AC), large cell neuroendocrine carcinoma (LCNEC) and small cell lung cancer (SCLC). RESULTS We performed 20 pneumonectomies, 13 bilobectomies, 3 sleeve-lobectomies, 75 lobectomies, 2 segmentectomies, 33 wedge resections, 1 bronchotomy. Pathological examination revealed: 9 tumorlets, 61 TC, 28 AC, 15 LCNEC, 34 SCLC. Mean follow-up was 67 months (range: 0-253 months). Tumor-specific 5-year survival was: $98 \%$ for TC, $91 \%$ for AC, $53 \%$ for LCNEC, 36\% for SCLC ( $\mathrm{p}<0,05)$; $98 \%$ for N0 and $100 \%$ for N1-N2 TC; $90 \%$ for N0 and 73\% for N1-N2 AC ( $<<0,05)$; $53 \%$ for N0 and 50\% for N1-N2 LCNEC; $56 \%$ for N0 and 19\% for N1-N2 SCLC $(\mathrm{p}<0,05)$.

Conclusion: Histological type and lymph node status were the most important prognostic factors in patients submitted to lung resection for NET; therefore, typical lung resections with mediastinal lymphadenectomy should be accomplished in all histological types.

\section{7}

Role of Positron Emission Tomography (PET) as Malignant Predictor in Thymic Epithelial Tumors

\section{G. Rizzardi, L. Bertolaccini, A. Terzi}

Thoracic Surgery Unit, S. Croce e Carle Hospital, Cuneo, Italy

Introduction: We want evaluate $\mathrm{PET} / \mathrm{CT}$ as predictor of pathological stage of thymomas.

Methods: Twenty-three patients were assessed by PET-CT before treatment. Maximum standardized uptake value $\left(\mathrm{SUV}_{\max }\right)$ and tumour $\mathrm{SUV}_{\max }$ /background mediastinal $\mathrm{SUV}_{\max }\left(\mathrm{SUV}_{\mathrm{T} / \mathrm{M}}\right)$ ratio were recorded. Thymomas were staged according to Masaoka, classified according to WHO, and divided in two groups: low-risk thymoma (LRT), A, AB and B; high-risk thymoma (HRT), B2, B3 and carcinoma. T-tests, U-test and Fisher's Exact Test were used for evaluating differences between group means. Correlations was evaluated using Spearman-rank test $(r)$, Kendall's $(\tau)$ and Goodman-Kruskal $(\gamma)$ correlations. We define as significant $p<0.05$, and highly significant $p<0.01$. We define as highly statistically significant correlation coefficients ranging $0.50-1.00$.

Results: Stage of disease was: I $(n=6)$, II $(n=8)$, III $(n=5)$, IV $(n=4)$. Differences between means of $S U V_{\max }$ were statistically significant, but differences between means of $S U V_{T / M}$ were not statistically significant. Highly statistical correlation between $\mathrm{SUV}_{\max }$ $(r=0.83, \tau=0.67, \gamma=0.74), \operatorname{SUV}_{\mathrm{T} / \mathrm{M}}(r=0.89, \tau=0.71, \gamma=0.72)$ and stage of disease was found. WHO thymoma's distribution was: A $(n=3) ; A B(n=5) ; B 1(n=4) ; B 2(n=2) ; B 3(n=2) ; C(n=7)$. Patients were divided respectively in LRT $(n=12)$ and HRT $(n=11)$. Despite small statistical sample differences between means of $\mathrm{SUV}_{\max }$ were highly significant $(p<0.0001)$ and between means of $\mathrm{SUV}_{\mathrm{T} / \mathrm{M}}$ were highly significant $(p<0.0069)$, and a correlation between $\mathrm{SUV}_{\max }$ $(r=0.88, \tau=0.66, \gamma=0.83)$ and $\operatorname{SUV}_{\mathrm{T} / \mathrm{M}}(r=0.91, \tau=0.79, \gamma=0.91)$ was found.

Conclusion: $\mathrm{SUV}_{\mathrm{T} / \mathrm{M}}$ is directly correlated to advanced stage of disease and higher degree of malignity. $\mathrm{SUV}_{\mathrm{T} / \mathrm{M}}$ might be used to identify patient with HRT and could modify clinical management decisions.

\section{8}

\section{Persistent Spontaneous Pneumothorax Associated with an Azygos Lobe: A Case Report}

M. Pucci, E. Romani, P. Bartolucci, L. Valesini, V. Usai, A. D'Amore, F. Cicconetti

U.O.C. Chirurgia dUrgenza e Trauma A, Dipartimento Emergenza Accettazione, Policlinico Umberto I, La Sapienza Università Roma, Italia

The azygos lobe is a common malformation: it has a reported incidence of $0,4-1 \%$. The association between a spontaneous pneumothorax and an azygos lobe is surprisingly rare. The Authors 
report a case observed in a 30 years old male smoker with persistent spontaneous pneumothorax after chest drain placement: due to persistent air leakage and no x-ray evidence of lung re-expansion, he underwent video-assisted thoracoscopic surgery treatment with bullectomy. VATS is increasingly used for management of a spontaneous pneumothorax, with recent reports suggesting its superiority to open thoracotomy.

\section{9 \\ VATS and Nd-YAG Laser vs Simple Chest Drainage for Treatment of Primary Spontaneous Pneumothorax}

\section{Pucci, E. Romani, P. Bartolucci, L. Valesini, V. Usai, F. Cicconetti \\ U.O.C. Chirurgia dUrgenza e Trauma A, Dipartimento Emergenza Accettazione, Policlinico Umberto I, La Sapienza Università Roma, Italia}

Primary spontaneous pneumothorax (PSP) is an extremely frequent pathology. Despite this, there is still no clear consensus on managing these patients. Aim of this study is to review our experience of treatment of PSP.

Methods: From the January, 2006 till April, 2009, 65 consecutive patients with PSP were treated with chest tube placement. 59 were men $(90.8 \%)$ and 6 were women $(9.2 \%)$ with a mean age of 33.8 years (range 15-75 years). 18 cases, due to persistent air leakage and/or not x-ray evidence of lung re-expansion, underwent VATS treatment: 11 with thoracoscopic bullectomy, 5 with Nd-YAG laser cauterisation of the blebs or bullae, 8 with pleural abrasion, 6 with videothoracoscopic talc pleurodesis, 1 with simple talcage after VATS, 1 with Nd-YAG laser pleurodesis. VATS treatment was undertaken after a mean of 8,83 days $(\min 2$, max 18) from chest drainage placement.

Conclusion: We conclude that VATS has superior outcomes in terms of recurrence rates of pneumothorax $(0 \%$ for VATS vs. $13,7 \%$ for tube thoracostomy alone, during 1-36 months of follow up). Duration of chest tube drainage (CTD) was shorter for patients without VATS treatment (6,0 for VATS vs. 4,9 days for CTD), even for mean hospital stay (16 days for VATS vs. 6 days for CTD). It is concluded that thoracoscopy enables accurate assessment of the causes of pneumothorax, talc pleurodesis is a very effective method of controlling persistent pneumothorax, and laser therapy can play an important role in selected patients with spontaneous pneumothorax.

\section{0}

Does Anatomical Segmentectomy Allow an Adequate Lymph Node (N) Staging for Non Small Cell Lung Cancer and for Brochopulmonary Carcinoids?
A. Ruffato ${ }^{1}$, S. Mattioli', L. Ferruzzi', V. Pilotti', M. Mandrioli ${ }^{1}$, P. Candoli ${ }^{2}$, F. D O $^{\prime}$ vidio $^{3}$
'Division of Thoracic Surgery, Villa Maria Cecilia Hospital, Cotignola (Ravenna),
Alma Mater Studiorum - University of Bologna,
2Division of Pneumology, Lugo Hospital, AUSL Ravenna,
${ }^{3}$ Division of Cardiothoracic Surgery, Columbia University, New York, USA

Objectives: Anatomical segmentectomy (S) is again under evaluation for T1N0 NSCLC and pulmonary carcinoids. Whether (S) does permit or not an adequate resection of nodal stations for staging or cure is still pending.

Methods: 39 patients (68\% men) underwent (S) (19 Adenocarcinomas 49\%, 14 Epidermoid Ca 36\%, 4 BAC 10\%, 2 carcinoids 5\%) versus 56 patients (76\% men) underwent lobectomy (L) (34 Adenocarcinomas 61\%, 14 Epidermoid Ca 25\%, 7 BAC $12 \%, 1$ undifferentiated Ca $2 \%$ ) for cT1N0M0 Ø max $2 \mathrm{~cm}$ at preoperative CT. Linfoadenectomia di principio delle stazioni 4,5 , 6, 7 in (S e L). Dissection of lymphnode stations 4, 5, 6, 7 during (S) and (L) was performed.

Results: Mortality was zero, median follow-up was: (S) 24 months (IQR 4.2-49.7), (L) 31 months (IQR 12-67.2). Median size of resected lesions was $1.8 \mathrm{~cm}$ (IQR $1.5-1.9 \mathrm{~cm}$ ) in $(\mathrm{S})$ and 1.8 $\mathrm{cm}(\mathrm{IQR} 1.5-2 \mathrm{~cm})(\mathrm{p}=0.2)$ in $(\mathrm{L})$. Resection margins were free in $100 \%$. Mean number $( \pm S D)$ of dissected lymph nodes was $9.5 \pm 3$ in $(\mathrm{S})$ compared to $17 \pm 7$ in $(\mathrm{L})(\mathrm{p}=0.001)$ with a median number of $\mathrm{N} 1=6(\mathrm{IQR} 3.7-7.2)$ and 8 (IQR 7-13.5) $(\mathrm{p}=0.01)$, and $\mathrm{N} 2=5$ (IQR 3.2-7.2) and 6 (IQR 3.5-10.5) (p=0.1). 1/39 (2.5\%) (S) was N1, while in (L), $5 \%$ was N1, 5\% N2 (3 pts).

Conclusions: (S) procures a lower number of N1 and equivalent number of $\mathrm{N} 2$ nodes for pathological examination. A potential under-staging (for N1) was noted in $2.5 \%$ of patients.

\section{1}

\section{Video-assisted Cervical Mediastinoscopy: Experience}

\author{
F. Colaut*, G. Fusco ${ }^{\circ}$ A. Sartori ${ }^{\wedge}$, L. Toniolo* \\ * Chirugia Toracica, P.O. Castelfranco Veneto, ^ U.O. \\ Chirurgia Generale P.O. Castelfranco Veneto, 'Chirurgia \\ Generale Università "Sapienza" Roma
}

\section{Chirurgia Toracica}

The cervical mediastinoscopy is still the god standard for staging the mediastinum for lymph nodes. Advances in technology have spread the use of mediastinoscopy, which increased security following the procedure econsentito alla'equipe under the control 
of sight. The video presents a mediastinoscopy performed with optical $5 \mathrm{~mm} 0^{\circ}$, we have called video-assisted mediastinoscopy. The method combines the ability to use the video support to the simplicity of the instruments. It is not excluded that the procedure will be improved in future.

\section{2 \\ Sleeve Lobectomy Versus Pneumonectomy for Non-small Cell Lung Cancer (NSCLC) with N1 Disease: Analysis of Short- and Long-Term Results and Pattern of Recurrence}

S. Marco, Z. Andrea, M. Giuseppe, D.A. Fabio, B. Lucia,

R. Alessandro, C. Francesca, R. Federico

Department of Cardiac, Thoracic and Vascular Sciences, Thoracic Surgery Division, University of Padova, Medical School

1st Classified - SPIGC Award Session, Thoracic Surgery

Objective: Sleeve lobectomy represents a valid alternative to pneumonectomy in locally advanced tumours. N1 disease is considered an important risk factor for local recurrence, especially for sleeve resections.

Aim of our study is to compare short- and long term results of sleeve lobectomy and pneumonectomy for NSCLC with N1 involvement, focusing on pattern of recurrence.

Methods: A retrospective study was carried out by reviewing 72 consecutive patients submitted to sleeve lobectomy for NSCLC-N1 at our Institution from 1990 to 2009. This group was compared with 84 consecutive patients who underwent pneumonectomy for the same pattern of disease in the same period. All patients were staged according to the $7^{\text {th }}$ edition of TNM.

Results: Both groups were similar for age, sex and pathologic stage of the patients.

In sleeve and pneumonectomy group perioperative mortality was $1.4 \%$ and $2.4 \%$, respectively $(\mathrm{p}=\mathrm{NS})$, while morbidity was $34.7 \%$ and $44 \%$, respectively ( $\mathrm{p}=\mathrm{NS}$ ). Overall 5-year survival was $48 \%$ and $44.5 \%$, respectively ( $\mathrm{p}=\mathrm{NS}$ ).

Loco-regional recurrences occurred in $14.1 \%$ and $8.5 \%$, respectively ( $\mathrm{p}=\mathrm{NS}$ ), while distant recurrences were found in $30.6 \%$ and $31 \%$, respectively $(\mathrm{p}=\mathrm{NS}$ ). We identified positive bronchial margin as risk factor for local recurrence $(\mathrm{p}=0.001)$ and adenocarcinoma histology for distant recurrence in both groups.

Conclusions: In our experience sleeve resection and pneumonectomy in N1 disease achieved comparable early and long term results, in particular for the rate of recurrence (local and distant). Positive bronchial margin and adenocarcinoma histology were risk factors for recurrence. For these reasons, N1 disease does not preclude sleeve procedures.
263

\section{Catamenial Pneumothorax After Fivet Procedure: Report of a Case}

A. Stanzi, F. Raveglia, A. Leporati, A. Rizzi, A.M. Calati, A. Baisi

Azienda Ospedaliera San Paolo - Milano, Università degli Studi di Milano, USD Chirurgia Toracica

Introduction: Catamenial PNX is a unique entity occurring in women at the onset of menses. The video reports a case of PNX after hormonal treatment.

Methods: A 32 year old woman presented with right PNX. She had right back noise and cough every menses. She underwent unsuccessful FIVET 2 weeks before. At thoracoscopy we found tiny blue-brown lesions on dorsal pleura and diaphragm,but no holes on it. We found open blebs at the apex of the inferior lobe and removed them. No endometriosis was identified on this specimen. Endometrial foci were coagulated. Pleural abrasion was also performed.

Results: No recurrence of PNX or back noise occurred after 22 months follow-up. She underwent a new successful FIVET.

Conclusions: Different theories exist to explain thoracic endometriosis:we found endometriosis on diaphragm and parietal pleura supporting the implantation theory. Many hypothesis exist to explain the relationship between endometriosis and PNX:air passing through diaphragmatic fenestrations, rupture of blebs due to PGF2,or visceral pleura perforation due to subpleural endometriosis proliferating at menses. In our case blebs rupture,due to hormonal storm preparing FIVET,was the unique explanation. We conclude that surgical bleb resection,endometriosis coagulation and pleurodesis may be appropriate to prevent relapse and thoracic pain related to pleural endometriosis during menses.

264

\section{Surgical Treatment of Endothoracic Goitres:} Our Experience

D. Angela, T. Francesca, *Loizzi Domenico,

*Sollitto Francesco, Loizzi Michele

Chirurgia Toracica

Introduction: Endothoracic goitres are usually treated with surgical excision, especially in symptomatic cases (main airways obstruction, thyrotoxicosis, etc). We report a retrospective study of surgical resection of endothoracic goitres.

Methods: From January 2005 to April 2010, 11 patients (7 females, 4 males; mean age 61,7 years, range: $43-75)$ were referred to our Institution for resection of suspected endothoracic goitres. We performed complete endothoracic goitres excision through the following surgical accesses: 6 cervicotomy with sternal split, 2 cervicotomy with total sternotomy, 2 cervicotomy, 1 cervicotomy associated to lateral thoracotomy.

Results: Post-operative period was uneventful in all patients but one, who required ICU stay and mechanical ventilation. All

Eur Surg Res 2010;45:158-307 
minor complications resolved with medical treatment ( 1 increase of transaminasemia and lipasemia, 1 hypocalcemia, 2 acute renal failure, 1 atrial fibrillation, 2 asmathic symptoms). Histological examination revealed thyroid struma in all cases (in 2 associated with papillary thyroid carcinoma).

Conclusion: Surgical resection of endothoracic goitres usually allows to obtain complete resolution of the disease with few complications. According to the dimension and site of growth of the goitre, surgical excision is generally accomplished through a cervicotomy, cervico-sternotomy or cervico-thoracotomy.

\section{5 \\ Long Term Results of Sleeve Lobectomy for Non-Small Cell Lung Cancer}

\section{A. Viti, F. Davini, M. Lucchi, A. Mussi \\ Unità Operativa di Chirurgia Toracica, Dipartimento Cardiotoracico, Università di Pisa (Direttore Prof. Alfredo Mussi)}

Introduction: Sleeve lobectomy has demonstrated effective local control and comparable long term survival compared with pneumonectomy in the treatment of non-small cell lung cancer (NSCLC). The objective of this study is to report our experience with sleeve lobectomy for NSCLC in terms of mortality, morbidity, and long term survival.

Methods: We retrospectively reviewed 50 patients who underwent sleeve lobectomy for NSCLC between January 2001 and December 2006. There were 48 men and two women, with a mean age of 64 years (range 50-80 years). Eight patients (16\%) received neo-adjuvant chemotherapy.

Results: There was 1 post operative death (2\%) while post operative morbidity rate was $14 \%$. Two patients experienced bronchopleural fistula. Univariate analysis demonstrated that neoadjuvant therapy did not affected the incidence of postoperative complications $(\mathrm{p}=0,1)$, while $\mathrm{N} 1$ disease was related with a higher rate of postoperative complications $(\mathrm{p}=0,01)$. The five years survival rate was $45 \%$.

Conclusions: Sleeve lobectomy can be performed with minimal bronchial anastomotic complications and low postoperative mortality. In our study, N1 disease adversely influenced the rate of postoperative complications.

\section{6}

\section{Radio-Guided Localization of a Small Pulmonary Nodules During Thoracoscopic Resection}

\author{
C. Zirafa, F. Melfi, A. Picchi, F. Allidi, A. Mussi
}

Thoracic surgery Division, Cardiac Thoracic and Vascular

Department, University of Pisa, Italy

Introduction: Today Video-Assisted Thoracic Surgery (VATS) is the procedure to prefer for diagnosis and treatment of pulmonary nodules. However, small or not peripheral nodules can be invisible or impalpable during toracoscopy, so difficult to localized. Many techniques have been developed to solve such problem. Herein we report our 13-years experience with Radio-guided One.

Materials and Method: Patients with pulmonary nodules smaller than $1 \mathrm{~cm}$ and/or deeper, below the visceral pleura, underwent CT-guided injection, into the nodule a solution composed by $0,2 \mathrm{ml}$ 99Tc- labelled human serum albumin microsphers and 0,1 $\mathrm{ml}$ non-ionic contrast. Then, during the toracoscopic procedure, a $11 \mathrm{~mm}$ diameter-collimated probe connected to a gamma ray detector is introduced to scan the lung surface. The area of major radioactivity is resected.

Results: From 1997 to 2009, 450 patients underwent toracoscopic resection of a small pulmonary nodules, 155 with the radio-guided technique. The patients were men and women, with average age of 60,6 (range: 12-83). Mean duration of surgical procedure was $43 \mathrm{~min}$ (range: 20 -100). The procedure was successful in $150 / 155$ cases. Five cases $(3,2 \%)$ required conversion to minithoracotomy approach. The mean length of pleural dreinage and hospital stay was 2,3 and 4 respectively. Histological examination showed 75 benign lesions and 80 malignant lesions (43 metastasis and 37 primary lung cancers).

Conclusions: Localization by gamma probe, after injection of 99-Tc, and resection of pulmonary nodules in VATS is a safe and quick with a high rate of success.

267

Treatment of Spontaneous Pneumothorax by Video-Assisted $3 \mathrm{~cm}$ Axillary Minithoracotomy

L. Domenico*, D. Angela, G. Giuseppe, B. Teresa,
L. Michele, S. Francesco*

Cattedra di Chirurgia Toracica, Università degli Studi di Bari, "Cattedra di Chirurgia Toracica, Università degli Studi di Foggia

Introduction: The Authors report a retrospective study of cases of primary spontaneous pneumothorax (PSP) referred to our Institution during the last decade and treated with simple pleural drainage or surgical resection by video-assisted axillary minithoracotomy $(3 \mathrm{~cm})$ (VAAMT). 
Methods: From January 1998 to December 2008 we treated 253 patients. Sex, age, type of treatment, hospital stay and recurrence rate were analyzed. We performed simple pleural drainage in case of first episode PSP (34 pts) and wedge resection with mechanical pleurodesis in VAAMT in case of second episode or prolonged air leaks (219 pts). Median follow-up was 54 months.

Results: The analysis of cases showed the following Results: $\mathrm{M}: \mathrm{F}$ ratio $=6: 1$; median age 22 years (range $15-38$ years); median hospital stay for patients treated with simple pleural drainage 5 days (range: 4-9 days), with ipsilateral recurrence rate of $32 \%$; median hospital stay for patients surgically treated 7 days (range 6-11 days), with ipsilateral recurrence rate of $0.9 \%$.

Conclusion: We recommend pleural drainage after first episode PSP and surgery after second episode or in case of prolonged air leaks as treatment of choice. VAAMT is a safe and effective technique, with low ipsilateral recurrence rate $(0.9 \%)$ compared with literature data concerning video-assisted thoracoscopic surgery (ipsilateral recurrence in $7-10 \%$ of cases).

268

\section{Use of Lactosorb Plates in Chest Wall Reconstruction Surgery for Congenital and Post-Traumatic Malformations. Preliminary Results}

\author{
L. Domenico, A.N. Pia*, D. Angela*, L. Michele*, \\ S. Francesco
}

Università degli Studi di Foggia, Dipartimento di Scienze Chirurgiche, Sezione di Chirurgia Toracica, Direttore Prof. Francesco Sollitto; *Università degli Studi di Bari, Sezione di Chirurgia Toracica Direttore Prof. Michele Loizzi.

Introduction: Chest wall deformities represent a heterogeneous group of defects affecting young patients as congenital malformation or adults as the result of a thoracic trauma. We present our preliminary experience on lactosorb plates apposition for chest wall congenital asymmetric depressions and post-traumatic flail chest stabilization.

Methods: Two 14 year old male patients with unilateral depression of left anterior chest wall were submitted to resection of depressed cartilagineous segments; they were replaced by previously heat modeled lactosorb plates (2x19 holes, $1 \mathrm{~mm}$ thick; Lorenz Plating System, Lactosorb, Biomet Microfixation, Jacksonville, FL). The plates were fixed at the tips to the sternum and to the residual ribs by resorbable lactosorb screws and sutured to the pericondral beds.

A 44 year old male patient with post-traumatic right flail chest was submitted to chest wall stabilization using lactosorb plates fixed by lactosorb screws and sutured to fractured ribs.

This material do not need to be removed as it's completely resorbable within one year.

Results: Two patients needed a chest drainage. No complications occurred. Mean post-operative stay was 4.6 days. Aesthetic results were satisfactory.
Conclusion: Surgical apposition of lactosorb plates could be successfully used for the correction of congenital and acquired chest wall deformities.

269

A New Dry, Portable, Digital System for Pleural Drainage Management. Preliminary Experience

\author{
L. Domenico, A.N. Pia*, D. Angela*, L. Michele*,
} S. Francesco

Università degli Studi di Foggia, Dipartimento di Scienze Chirurgiche, Sezione di Chirurgia Toracica, Direttore Prof. Francesco Sollitto; *Università degli Studi di Bari, Sezione di Chirurgia Toracica Direttore Prof. Michele Loizzi.

Introduction: Pleural drainages contain a water seal, mechanic or dry unidirectional valve for gas and fluids evacuation. We report preliminary results on the use of a dry, portable, digitally controlled system for pleural drainage.

Methods: We adopted Medela Thopaz system in 16 consecutive patients submitted to lobectomy $(n=4)$, bilobectomy $(n=2)$, wedge resection, $(n=5)$, decortication $(n=3)$, thymectomy $(n=1)$ and drainage for pneumothorax $(n=1)$. Except for pneumothorax, two chest tubes were positioned.

Aspiration was electronically set up from -8 to $-10 \mathrm{cmH} 2 \mathrm{O}$. Air leak diagram was digitally acquired for every patient. Chest tubes were removed with daily collected fluid $<150 \mathrm{ml}$ and no air leaks documented in the last 24 hours, after chest X-ray.

Results: Mean chest tube duration was 4.7 days. Mean postoperative stay was 7.5 days. Deambulation was possible at the first post-operative day. No significant complications occurred.

Conclusion: This drainage system allows air leak digital monitoring and recording, is portable, has a rechargeable battery, gives acoustical and optical signals that make it safe and reliable. Aspiration works only when air flow is registered. This system could improve outcome in thoracic surgery, promoting patient's mobilization, reducing chest tube duration and post-operative stay. More experience is needed to reduce mistakes in management of this device. 
Vascular Surgery

270

Mycotic Extracranial Internal Carotid Artery Aneurysms: Case Report and Review

C. Busoni, A. Odero, A. Popovich, E. Casabianca, G. Poletto, P.L. Giorgetti

${ }^{1}$ Scuola di Specializzazione in Chirurgia Vascolare Università degli Studi di Pavia, ${ }^{2}$ U.O. Chirurgia Vascolare 1 IRCCS Istituto Clinico Humanitas - Rozzano (MI)

Introduction: Extracranial internal carotid artery aneurysm (EICA) are rare and difficult to diagnose. Therapeutic options and prognosis are different in true EICA, mycotic and pseudo-aneurysms.

Materials and Methods: We describe an EICA case related to an infective colonization: a woman carring a left huge laterocervical painless mass, in progressive increasing diameter after 10 years from fever and malaise during holidays in Africa. Recently she was submitted to echo guided biopsy in suspicion of salivary glands or lymphnode neoformation but EICA was diagnosed: in order to anamnestical reports mycotic origin was suspected. Angio Tc and MR identified a $38 \mathrm{~mm}$ saccular EICA arising $2 \mathrm{~cm}$ above carotid bifurcation: aneurismal neck was of few millimetres; distal internal carotid was elongated due a kinking.

Results: Because of high infective risk and favourable anatomical feature, she was submitted to open surgery with aneurysmectomy and end-to-end anastomosis between proximal and distal internal carotid artery without any prosthesis interposition. At the histological examination no infective infarction was found, but only lymphnode aspecific reaction. In the post-operative period no neurological symptoms nor cranial nerve injury nor ischemic cardiac evidence were noticed.

Conclusion: According to anamnestical reports and aneurismal morphology we suspected a mycotic aetiology: the treatment of choice actually is surgical operation, in association with antibiotics therapy, because of the high risk of thrombosis, embolization and/or rupture. In particular, excision and reconstruction without prosthesis or, best, with autologous vein interposition graft, is the most definitive repair and has the benefit of removing the source of the infection.
271

\section{Does Durability Challenge the Role of Carotid Artery Stenting (CAS)? Comparison of 5 Years Results of CAS and Carotid Endarterectomy (CEA) in 2202 Patients}

A. Casalino, M. Barbante, P. Bonanno, D. Brambilla, P. De Rango

Vascular and Endovascular Surgery, University of Perugia

Introduction: The aim of this study was to evaluate outcomes of CAS, performed with current technique, compared to Carotid Endarterectomy (CEA)

Methods and Results: Consecutive patients with primary carotid stenosis treated by CEA or by CAS after a training phase were reviewed. 30 day (stroke, death, myocardial infarction, local) and 5-year complications composite of any perioperative stroke or death and any ipsilateral stroke after the procedure were compared. Data were stratified by symptoms. 1084 CAS and 1118 CEA performed in a single center during the period '01-'09 were compared. 30 day stroke and death rates were similar: $2.9 \%$ in CAS and $2.6 \%$ in CEA $(\mathrm{p}=0.27)$. Symptomatic patients $(\mathrm{n}=684)$ showed increased periprocedural stroke/death (PSD) risk (4.5\%vs $2.9 \%$, in CAS and CEA, respectively; $\mathrm{p}=0.29$ ) compared to asymptomatic patients $(2.3 \% \mathrm{vs} 1.6 \%$ in CAS and CEA; $\mathrm{p}=0.36)$ without significant difference. Five-year survival rates were $82 \%$ in CAS and $87.7 \%$ in CEA $(p=0.05)$. Life-table estimates of any (PSD) and ipsilateral stroke at 5 years after the procedure were similar, in all patients (4.4\%vs3.7 \%, in CEA and CAS, respectively; $\mathrm{p}=0.1)$ and the subgroups of symptomatic $(8.5 \% \mathrm{vs} 4.9 \% ; \mathrm{p}=0.56)$ and asymptomatic $(4.4 \% \mathrm{vs} 3.7 \% ; \mathrm{p}=0.4)$ patients.

Conclusion: CAS with updated techniques can offer efficacy and durability comparable to CEA with benefits persisting at 5 years.

272

\section{Endovascular Treatment of Iliac Aneurysms Involving the Hypogastric Artery: Concurrent Comparison of Branched Endografts Vs Hypogastric Occlusion}

D. Brambilla, A. Casalino, V. Tavolini, V. Silvestri, G. Parlani

Vascular and Endovascular Surgery, University of Perugia

Objective: To analyze early and midterm outcome of endovascular treatment in patients with iliac aneurysms,comparing the results of hypogastric revascularization by iliac branch endografting vs hypogastric occlusion.

Methods: Consecutive patients with iliac aneurysm receiving side branch endograft and those with hypogastric exclusion,in 10 years,were compared.

Results: 104 patients were treated and classified in two Groups. No difference in risk factors and aneurysm iliac diameter were found. Concurrent treatment of aortic aneurysm was performed in $78 \%$ vs $81 \%$. In patients with bilateral iliac involve- 
ment hypogastric artery was saved or revascularized. No significant differences in failures of hypogastric side branch deployment. During follow up iliac occlusions rates were similar in 2 Groups but no occlusions of hypogastric side branch occurred. No difference in reintervention. Endoleak was present in $17 \%$ in Group 2 and $2,1 \%$ in Group 1. Buttock claudication or impotence were more frequent in group 2.

Conclusions: Endovascular treatment of iliac aneurysm with hypogastric revascularization through side branched endografts is feasible and safe. Endoleak and buttock claudication occur frequently in patients with hypogastric exclusion. Side branch endografting for iliac aneurysm may be considered a primary choice in younger and active patients with suitable anatomy.

\section{3 \\ Functional Ability in Patients with Critical Limb Ischemia Is Unaffected by Successful Revascularization}

G. Isernia, A. Marucchini, V. Silvestri, M. Barbante, M. Lenti

Vascular and Endovascular Surgery, University of Perugia

Introduction: The aim of the study was to investigate whether the limitations encountered in the activities of daily living (ADL) measured with the Katz Index (KI) in patients with CLI were changed by the treatment.

Methods: During the period '06-'08, 278 patients undergoing repair for CLI were investigated with an ADL questionnaire for assessing KI before and after 1 month from treatment. Changes in KI were stratified by type of treatment and outcome.

Results: There were 201 males and 77 females, mean age $74.6 y e a r s ; 145$ patients showed tissue loss and 133 rest pain. 126 received surgical bypass (OR) and 152 endovascular repair (EP). KI preop index showed that patients were dependent in the majority of functions. At the postop assessment there was significant worsening in patients functional outcome, despite relief of pain (85\%), tissue healing (81\%) and good vessel patency $(74 \%)$ and low amputation rate $(6 \%)$ at 36 months. Deterioration of KI was similar in patients undergoing OR and ER. Despite a higher baseline dependence status, patients receiving major amputation did not further deteriorate. Patients who received successful revascularization showed a significant deterioration in KI.

Conclusions: Successful treatment may not be associated with improved functional ability in patients with CLI often highly dependent in most of their activities. Use of endovascular treatment has similar detrimental effect than open surgery.

\section{4}

\section{Mid-Term Results in Election Treatment for Popliteal Artery Aneurysms}

\section{P. Bonanno, V. Silvestri, A. Casalino, G. Isernia, L. Romano \\ Vascular and Endovascular Surgery, University of Perugia}

Introduction: The aim of the study is to evaluate the immediate and mid-term results for elective surgical treatment for popliteal aneurysms (AP), with posterior access (PA) or medial access (MA), in relation with the use of autologous (AG) or synthetic graft (SG).

Methods and Results: 101 (AP) in 89 patients ( $85 \mathrm{M}, 4 \mathrm{~F}$ ) were electively treated, the average age was 69.71 years, the average diameter was $29,47 \mathrm{~mm}$. 42 cases treated with PA: 34 (AG) (84\%), 2 "end to end"; 9 ePTFE graft, 1 other biological graft. 59 cases with AM: 35 (AG), 24 ePTFE graft. There were not deaths and/or amputations at 30 days, and technical success was achieved in $100 \%$. The average follow-up was 14.34 months. Actuarial primary patency for PA and MA was $100 \%$ at 42 months and $78 \%$ a 42 months. Primary patency for syntetic graft was $67 \%$ at 35 months against $95 \%$ at 42 months for autologous vein graft $(\mathrm{P}=$ $0,015)$. Assisted primary patency for MA was $81 \%$ at 42 months; in this group assisted primary patency for syntetic graft was $75 \%$ at 35 months against $95 \%$ at 42 months for autologous vein graft $(\mathrm{p}=0,043)$.

Conclusions: Primary patency in the medium term was not influenced by graft type in PA. In the MA autologous graft maintains, in terms of primary patency, an advantage over the synthetic. Aneurysm diameter did not influence the rate of complications and patency.

\section{5}

\section{Does Durability Challenge the Role of Carotid Artery Stenting (CAS)? Comparison of 5 Years Results of CAS and Carotid Endarterectomy (CEA) in 2202 Patients}

\section{A. Casalino, M. Barbante, P. Bonanno, D. Brambilla, P. De Rango \\ Vascular and Endovascular Surgery, University of Perugia}

Introduction: Randomized Controlled Trials (RCTs) have questioned the efficacy and durability of Carotid Angioplasty and Stenting (CAS) but the quality of CAS procedures performed in most trials is debatable and technique somewhat outdated. The aim of this study was to evaluate outcomes of CAS, performed with current technique, compared to Carotid Endarterectomy (CEA).

Methods: Consecutive patients with primary carotid stenosis treated by CEA or by CAS after a training phase were reviewed. Thirty-day (stroke, death, myocardial infarction, local) and 5-year complications composite of any perioperative stroke or death and any ipsilateral stroke after the procedure and recurrent carotid stenosis) were compared. Data were stratified by symptoms, age and gender. Mean follow-up was 33.05 months. 
Results: 1084 CAS and 1118 CEA (71\% males, mean age 71.3y) performed in a single center during the period 2001-2009 were compared. Thirty-day stroke and death rates were similar: $2.9 \%$ in CAS and $2.6 \%$ in CEA $(\mathrm{p}=0.27)$. Symptomatic patients $(\mathrm{n}=684)$ showed increased periprocedural stroke/death risk $(4.5 \%$ vs. $2.9 \%$, in CAS and CEA, respectively; $\mathrm{p}=0.29)$ compared to asymptomatic patients $(2.3 \%$ vs $1.6 \%$ in CAS and CEA; $\mathrm{p}=0.36)$ without significant difference between CAS and CEA. Five-year survival rates were $82 \%$ in CAS and $87.7 \%$ in CEA $(p=0.05)$. Lifetable estimates of any periprocedural stroke/death and ipsilateral stroke at 5 years after the procedure were similar, in all patients ( $4.4 \%$ vs $3.7 \%$, in CEA and CAS, respectively; $p=0.1$ ) and the subgroups of symptomatic ( $8.5 \%$ vs $4.9 \% ; \mathrm{p}=0.56)$ and asymptomatic ( $4.4 \%$ vs $3.7 \% ; \mathrm{p}=0.4)$ patients. Cox regression identified the lack of statin treatment $(\mathrm{P}<0.01)$ and symptomatic disease $(0.002)$ as positively associated with the composite end-point. Recurrent stenosis ( $>50 \%)$ was non-significantly more frequent in the CEA group, with a life-table estimate of $5.8 \%$ versus $3.4 \%$ in CEA and CAS $(p=0.7)$ at 5 years. There were no differences between CAS and CEA in periprocedural and long term risk in analyses stratified by gender and age.

Conclusions: CAS with updated techniques can offer efficacy and durability comparable to CEA with benefits persisting at 5 years. Symptomatic patients demonstrate increased stroke risk, whichever the treatment. CAS might not be discouraged as a primary choice for treatment of carotid stenosis in average risk patients. Long-term data from ongoing RCTs performed by skilled operators are awaited for a final conclusion.

\section{6}

\section{Use of Percutaneous Atherectomy of Lower Extremity Peripheral Arterial Disease in Diabetics}

\author{
D. Cecere, L. Scevola, R. lanni, P. Valitutti \\ Casa di Cura "Malzoni" Agropoli (SA)
}

Introduction: Endovascular options for the treatment of lower extremity peripheral arterial disease are typified with poor patency. A new alternative to angioplasty and stent is the excision of the obstructing arterial plaque using a minimally invasive technique, the Silverhawk atherectomy device.

Materials and Methods: From February 2009 to January 2010, 12 atherectomy procedures were performed on 8 limbs of 8 diabetics. The location of the vessel stenosis was the SFA in 8 and popliteal artery in 4 . Atherectomy was successful in 9 primary procedures and all 2 repeat atherectomy procedures, with adjuvant balloon dilation required in 1 case. It happened no other complications. The technical success rate was $100 \%$. In 4 cases, it was required to use the new Rockhawk device for the treatment of heavily calcified femoro-popliteal lesions. In 7 pts was employed distal embolic protection (SpiderFX).

Results: After 3, 6 and 12 months, primary/assisted primary patency rates per treated limb were $100 \% / 100 \%, 87,5 \% / 100 \%$, $75 \% / 100 \%$ respectively.
Conclusion: Compared to conventional PTA and stent implantation for arterial occlusive diseases, atherectomy offers the advantages of eliminating stretch injury on arterial walls and reduce the rate of restenosis in diabetics.

\section{7}

\section{Durability of Endovascular Treatment of Iliac Artery Occlusions}

\author{
A. Guidotti, F. Sangrigoli, M. Di Mare, V. Sanna, W. Dorigo, \\ G. Pratesi, C. Pratesi \\ Department of Vascular Surgery, University of Florence, \\ Italy
}

Objectives: To evaluate early and long-term results of endovascular treatment of iliac artery occlusions, and to compare these outcomes to those obtained in patients treated for stenotic lesions.

Methods: Over a 10 year period ending in 2009, 215 endovascular procedures for the treatment of peripheral arterial disease (PAD) with the involvement of aorto-iliac district were performed. The intervention was performed for the presence of iliac occlusion in 105 cases (TASC II B, C and D lesions, group 1) and of iliac stenosis in 110 patients (TASC II A lesions, group 2). Early and follow-up results were analyzed and compared.

Results: There were no differences in terms of intraoperative technical details and perioperative results. Mean duration of follow-up was 29 months (range 1-116). Estimated 60-month primary, assisted primary and secondary patency and survival rates in group 1 were $92 \%, 92 \%, 96 \%$ and $82.5 \%$, respectively, without significant differences with group 2 .

Conclusions: In our experience, endovascular treatment of iliac occlusions provides excellent early and long-term results, similar to those obtained in the treatment of stenotic lesions.

\section{8 \\ Early and One-Year Comparison Between Open and Endovascular Treatment of Popliteal Artery Aneurysms}

\author{
I. Spina, L. Paperetti, A. Fargion, R. Pulli, W. Dorigo, \\ C. Pratesi
}

Department of Vascular Surgery, University of Florence, Italy

3rd Classified - SPIGC Award Session, Vascular Surgery

Aim of the Study: To retrospectively compare perioperative ( $<30$ days) and one-year results of open and endovascular management of popliteal artery aneurysms (PAAs) in a single centre experience. 
Materials and Methods: From January 2005 to December 2009, 56 PAAs in 50 patients were operated on at our Institution; in 39 cases open repair was performed, while in the remaining 17 cases an endovascular procedure was attempted (Group 2). Early and follow-up results were compared.

Results: There were no differences between the two groups in terms of sex, age, risk factors for atherosclerosism comorbidities and clinical status. Cumulative 30-day death and amputation rates were $5 \%$ in group 1 and $6 \%$ in group $2(p=0.9)$. Cumulative 30 -day reintervention rate was significantly higher in group 2 than in group $1(\mathrm{p}=0.04)$. Mean follow-up time was 19.1 months (range 1-48); Estimated primary patency rates at 12 months were $78.9 \%$ in group 1 and $59 \%$ in group $2(p=0.1$; log rank 2.4$)$. Also secondary patency and limb salvage were similar among the two groups. Freedom from reintervention rates at 12 months were $87 \%$ in group 1 and $58.5 \%$ in group $2(\mathrm{p}=0.01$; log rank 6.1$)$.

Conclusions: Endovascular treatment of PAAs provided satisfactory perioperative and one-year results, not significantly different from those obtained with open repair even with a higher risk of immediate and late reintervention.

279

\section{Improvements in the Results of Combined Carotid and Cardiac Surgery}

\author{
M. Calistri, E. Chiti, S. Matticari, L. Azas, C. Pratesi \\ Department of Vascular Surgery, University of Florence, \\ Italy
}

\section{2nd Classified - SPIGC Award Session, Vacular Surgery}

Background: Aim of this study was to analyze our experience in the last five years of combined carotid and cardiac surgery.

Methods: During a 5-year period (January 2002-December 2006), 111 patients underwent combined carotid endarterectomy (CEA) and coronary artery bypass grafting (CABG) (Group 1), while 1446 patients underwent isolated CEA (Group 2). Perioperative and follow-up outcomes in the two groups were compared.

Results: There was no difference in the incidence of stroke ( $1 \%$ Group 1 vs $0.6 \%$ Group 2, p=n.s.). Mortality rate was increased in the combined surgery group, (3.5\% versus $0.5 \%, \mathrm{p}<0.001)$. Combined stroke/death at 30 days was $4.5 \%$ in Group 1 compared to $0.9 \%$ in Group 2, $\mathrm{p}=0.001$. Mean follow-up was 18.7 months (range 1-60). Survival at 24 months was significantly higher in patients of Group 2 compared to Group 1 (99.4\% vs $91.3 \%$ respectively, $\mathrm{p}<0.001)$. At 24 months, there were no significant difference between the two groups in the risk of developing ipsilateral or contralateral neurologic events (3.1\% Group 1 vs $1.7 \%$ Group 2).

Conclusions: Combined CEA and cardiac surgery carries a higher risk of perioperative mortality than patients undergoing isolated CEA.

\section{0}

\section{Short-term Patient-reported Outcomes of Open and Endovascular Treatment of Peripheral Arterial Disease}

E. Giacomelli, C. Pratesi, S. Taddei, E. Compiani, G. Pratesi, W. Dorigo, R. Pulli

Department of Vascular Surgery, University of Florence, Italy

1st Classified - SPIGC Award Session, Vascular Surgery

Objectives: To prospectively evaluate the effect of open and endovascular treatment of peripheral arterial disease (PAD) on perceived quality of life and health status in a single centre experience.

Material and Methods: From October 2008 to October 200994 patients undergoing open (34 patients, group 1) or endovascular (60 patients, group 2) intervention were prospectively enrolled in the study. The Peripheral Artery Questionnaire (PAQ) and SF-12 questionnaire were pre and postoperatively submitted to all these patients. Pre and postoperative data in the two groups were compared.

Results: Mean PAQ summary score and SF-12 physical component significantly improved after intervention. Patients in group 1 had a significant improvement in PAQ summary score, and in PAQ physical limitation, symptoms, symptom stability and quality of life subscales; patients in group 2 had a significant improvement in PAQ summary score and in all its subscales and also in SF-12 physical component.

Conclusions: Surgical intervention (open or endovascular) for peripheral arterial disease provides significant improvement in health status and quality of life at a short-term follow-up. Endovascular treatment seems to better improve treatment satisfaction, social limitation and general physical health perception.

\section{1}

\section{Surgical Treatment of Abdominal Aortic Aneurysm (AAA) Associated with Renal Anomalies}

\section{F. Giacchi, G. Tinelli, F. Snider}

Vascular Surgery Unit - Catholic University, Rome

Introduction: The treatment of abdominal aortic aneurysm (AAA) associated with renal anomalies (RA) involves specific technical-surgical problems. The principal RA are horse-shoe kidney (HSK) and ectopic kidney (EK). HSK is the most common RA associated with AAA (0.12\%).

Methods: Between January 1990 and March 2010 we operated 1026 AAA and 9 of this cases presented associated RA: 7 HSK and 2 bilateral EK. Renal vascularization was: normal renal arteries associated with isthmic arteries beginning from aortic branch ( 5 cases of HSK) and associated with small supernumerary arteries ( 1 case of HSK), dual left renal artery associated with dual infe- 
rior cava vein ( 1 case of HSK), triple right renal artery associated with left inferior polar artery (1 case of HSK) and common trunk beginning from aortic branch associated with dual inferior cava vein (1 case of pelvic EK). An extra-peritoneal sec. Sicard access in HSK cases (aorto-aortic bypass) and a transperitoneal xifo-pubic access in EK cases (aorto-bisiliac bypass) was performed.

Results: There was any vascular complications, a transient ipercreatininemy in 2 patients (sovrarenal clamping) and a bronco-pneumonic focus and jaundice in a patient, resolved with medical therapy.

Discussion: Surgical treatment of AAA associated with RA imposes an attentive planning of pre-operatory surgical strategy. Extraperitoneal access is preferable in AAA with HSK. Endovascular treatment of AAA with HSK allows the same principles recognized for isolated aneurismatic aortic pathology.

\section{2}

Aortic Fast Track Surgery: A Randomized Comparision of Perifascial Infusion Catheter and Local Anaestetic Infiltration
A. Odero, G. Poletto', E. Casabianca , A. Popovich, C. Busoni ${ }^{\circ}$, M. Spoto*, P.L. Giorgetti
¿UO Chirurgia Vascolare I - IRCCS Humanitas - Rozzano, *UO Anestesia Rianimazione Generale - IRCCS Humanitas - Rozzano, ${ }^{\circ}$ Cattedra di Chirurgia Vascolare Università degli Studi di Pavia

Objectives: Our experience in FT aortic surgery started in 2007 without changes in surgical technique;analgesia was assured with local anesthetic infiltration ${ }^{1,2}$. Aim of this study is compare our FT protocol to a novel analgesic approach with a perifascial infusion catheter.

Methods: A randomized prospective study was designed with 38 enrolled patients who underwent open aortic surgery with a FT protocol.18 patients were randomized to a 48 hours subfascial multi lumen catheter (ON-Q) continous local anaesthetic instillation with Naropine 1\%(GROUP A). 20 received our traditional FT protocol (GROUP B). Patients were mobilized few hours after intervention in group A and in I postop day in B; early iperproteic oral intake was encouraged, PONV was prevent by prokinetik drug.

Results: Both groups matched for sex and age (66.17 Vs $68,15)$ were similar; no statistical difference in mean intervention time ( $169 \pm 6$ Vs $159 \pm 4$ ), nor in pain VAS in 48 p.o. hours. No rescue analgesia was required. Bowel canalization was achieved in $55,7 \pm 2$ Vs 58,8 \pm 3 hour (p NS): 3 Vs 2 pt had nausea and 2 cases Vs 1 was adinamic ileus in each group. Mean discharge day was $4,7 \pm 1,3$ in group $A$ and $6 \pm 1,6$ in $B(p<0,05)$.

Conclusions: Our series demonstrate how a minimal analgesic technique change improve FT pathway, may reduce surgical stress and facilitates po mobilization with earlier achievement of clinical discharge criteria.

\section{3}

\section{Management of Late Endograft Failure}

\author{
M. Guillou, G. Kaur, F. Ramponi, D.V. Simring, \\ M.S. Stephen, J.P. Harris, S.R. Dubenec, R. Qasabian, \\ R. Waugh*, G.H. White
}

Department of Vascular Surgery, Royal Prince Alfred

Hospital, Sydney - Australia, " Department of Radiology,

Royal Prince Alfred Hospital, Sydney - Australia

Background: Endovascular aortic aneurysm repair (EVAR) has now become a well established technique worldwide. Later generation endograft devices continue to have significant rates of late failure.

Aims: The aims of this study were to analyze the modes of failure, methods of effective repair and outcomes over a recent 3-year interval.

Patients and Methods: We retrospectively reviewed the techniques and outcomes for 35 patients who had secondary procedures $(n=46)$ for management of device failure during the last 3 consecutive years.

Results: The average time between endograft implantation and the redo procedure was 4 years and 3 months. Six patients needed an open procedure. Among 29 patients who needed an endovascular procedures, coiling of type I or type II endoleaks was used in 13 patients, graft extensions in 11, and total endograft "relining" with a new device in 13. Three patients presented back with an acute aneurysm and underwent an endovascular procedure in 2 cases and an open in 1 case. Periprocedural mortality rate was $4 \%$.

Conclusions: Endograft failure is still encountered fairly frequently, although most cases are still related to device designs which are now obsolete. Secondary endovascular procedures can be done in the majority of cases, with low mortality.

\section{4}

\section{Familial Aortic Aneurysm and Dissection Due to TGF $\beta$ R2 Gene Mutation - Clinical Course and Current Management}

\author{
F. Ramponi', J. Edelman², R. Jeremy³, P.G. Bannon², \\ G.H. White ${ }^{1}$
}

${ }^{1}$ Department of Vascular Surgery, ${ }^{2}$ Department of Cardiothoracic Surgery, ${ }^{3}$ Department of Cardiology, Royal Prince Alfred Hospital, Sydney - Australia

Objective: This report describes the clinical course and management strategy for an individual affected by Loeys-Dietz syndrome types II (LDS-II), a familial condition characterized by aortic aneurysm and dissection, associated with mutations in genes for transforming growth factor receptors (TGF $\beta R$ ).

Case History: In September 2003, a 31 ys old healthy surfer, with a strong family history of thoracic aortic pathology, was diagnosed with acute type B aortic dissection; after initial medical management, he developed progressive hypertension second- 
ary to true lumen compression and renal ischaemia. Subsequently he underwent successful aortic balloon fenestration and stenting (Z-Stent Cook, Bloomington USA). In 2006, despite good blood pressure control, the patient developed symptomatic dilatation of the distal aortic arch and of the visceral abdominal aorta; he underwent successful surgical replacement of the arch and stenting of the descending thoracic aorta (Zenith Cook). In 2007 the patient underwent elective endoluminal repair of the infrarenal portion of the chronic dissection. The procedure was complicated by inadvertent deployment of the stent graft (Talent Medtronic) in the false lumen; a second graft had to be placed to lie alongside the other, with good final result. Shortly after discharge he experienced a short episode of lower limbs paresis and antiacoagulation was started. Genetic counseling in 2008 detected a bifid uvula and some hypermobility of the spine and knees joints (Breighton score 3/9) associated with a mutation in the TGF $\beta$ R2 gene; diagnosis of LDS-II was made. Despite optimal medical therapy the aortic root and abdominal aorta continued to enlarge and in 2009 the patient underwent uncomplicated root replacement. At that time the maximal diameter of the abdominal aorta was $65 \mathrm{~mm}$ above and $60 \mathrm{~mm}$ below the renal arteries. Further treatment will inevitably be required, with high risk of spinal cord ischaemia and paraplegia.

Discussion: LDS, described in 2005, is characterized by the triad of arterial tortuosity and aneurysms, hypertelorism and bifid uvula or cleft palate as a result of heterozygous mutations in genes encoding TGFßR 1 and 2 (1). Marfan Syndrome, Ehlers-DanlosIV and LDS-II have similar clinical presentations, but different natural history and prognosis, making accurate diagnosis essential (2). The mean age of death in LDS-I is 22.6 ys vs 31.8 ys in LDS-II; the latter has a similar age onset of EDS-IV, but a better surgical outcome. Few guidelines, based on aortic diameter and genetic mutation, suggest the correct timing for surgery. Features like severe craniofacial abnormalities (LDS-I) and positive TGF $\beta$ R2 mutation appear to be associated to a more aggressive disease and variable risk of concomitant malignancies $(3,4)$. Further studies are required to establish the proper surgical management and to elucidate the mechanisms of connective tissue failure.

\section{5}

Percutaneous Endovascular Aortic Aneurysm Repair With perclose Proglide Preclosing Technique

\section{F. Ramponi, D.V. Simring, M.S. Stephen, J.P. Harris, S.R. Dubenec, R. Qasabian, G.H. White \\ Department of Vascular Surgery, Royal Prince Alfred Hospital, Sydney - Australia}

Objective: Percutaneous access for endovascular aortic repair has been shown to be a feasible and safe procedure using suturemediated closure devices. The aim of this study was to report a single-centre experience of percutaneous femoral access during endoluminal aortic procedures using the Perclose Proglide device (Abbott Vascular, Redwood City, California) and to compare the early outcome with the standard surgical femoral exposure.
Methods: Between January 2009 and March 2010 a total of 117 patients underwent endovascular thoracic or abdominal aortic interventions (12F-25F sheaths). The approach to the femoral vessels was performed via surgical cutdown (54 pts), percutaneous "Preclose" technique (45 pts), or a combination of both (18 pts), resulting in 111 arteries accessed surgically and 97 percutaneously. Medical records and preoperative CT angiograms were retrospectively reviewed; rates of technical success, failure and complications in both groups were analyzed and compared, as well as procedural time and postprocedural length of stay in the hospital.

Results: A total of 163 Proglide devices were used to close 97 femoral arteries (1.68 device/artery); $18 \mathrm{~F}$ to $25 \mathrm{~F}$ sheaths were required in 67 cases $(69.1 \%)$, and sheaths less than $18 \mathrm{~F}$ in the remainder. There were 6 failures requiring femoral cutdown (6.18\%) for a technical success rate of $93.82 \%$; in 3 cases prolonged compression was necessary to control a minor haematoma $(3.1 \%)$. The rate of infection or late surgical revision of the access was $0 \%$. With respect to the surgical control group, the overall complication rate was 9\%: 6 cutdowns required surgical revision $(5.4 \%)$ and 4 were managed conservatively (3.6\%). Risk factors for failure of percutaneous access were: (i) the presence of femoral calcifications (7.69\% vs $3.12 \%, \mathrm{p}=\mathrm{ns})$; (ii) the use of a large diameter sheath $(8 \%$ vs $4.44 \%, \mathrm{p}=\mathrm{ns})$. Use of sheaths 18 to $25 \mathrm{~F}$ were also associated with higher rate of overall complications ( $14 \%$ vs $4.4 \%, \mathrm{p}=0.164)$. Higher BMI (>27.8) was not risk a factor for Proglide failure. Procedure time was compared between two similar subgroups of patients who underwent standard EVAR without any adjunctive procedure; the Preclose technique resulted in shorter procedure time (104 vs $115 \mathrm{~min}, \mathrm{p}=0.133$ ). Finally post-procedural hospital length of stay, excluding prolonged admissions for reasons not related with the access, was shorter in the percutaneous group (7.93 vs 3.94 days, $\mathrm{p}=0.006$ ).

Conclusions: Percutaneous endovascular aortic repair with Perclose Proglide is a safe and effective procedure, associated with shorter procedure time and post-operative length of stay. Femoral calcification, especially if associated with large sheath size, is a predictor of device failure; careful selection of patients is mandatory to obtain excellent technical success.

\section{6}

Criteria of Monitoring Graft Surveillance and Possibilities of Intervention in Failing Grafts: State of Art and Our Experience

\author{
A. Trani, A. Gasparre, B. Perretti, F. Bonanno \\ U.O.C. di Chirurgia Vascolare, Direttore: Dr. Francesco \\ Bonanno, Ospedale "Madonna delle Grazie", Azienda \\ Sanitaria Locale di Matera
}

Introduction: The objective of this study was to assess the prognostic value of hemodynamic parameters measured with duplex ultrasound scan, together with other important graft and patient characteristics, in predicting lower extremity vein graft thrombosis.

Materials and Methods: A total of 169 lower extremity vein grafts were entered prospectively into a postoperative 
duplex ultrasound scan surveillance program with examinations performed at $1,2,3,4,6,9,12,18$, and 24 months, and annually thereafter. Graft patency was determined after each visit.

Results: Thirty-two episodes of first-time graft thrombosis occurred, 23 of which were permanent. One-, 3-, and 5-year secondary graft patency rates were $90 \%, 86 \%$, and $79 \%$, respectively. In multivariate analyses, duplex scan velocity measurements predictive of lower extremity graft thrombosis included the maximum velocity ratio $(\mathrm{Vr})$ in association with a graft stenosis and the mean graft peak systolic velocity (MGV) within nonstenotic portions of the body of the graft.

The color PRF is usually set in the $2.5-3 \mathrm{kHz}$ range for demonstrating moderately high velocity flow. The incidence of graft thrombosis among grafts without inflow/outflow stenoses, with Vr less than 3.5, and with MGV $50 \mathrm{~cm} / \mathrm{s}$ or more, was $2.9 \%$ per year.

Conclusions: Duplex scan velocity measurements are valid predictors of impending graft thrombosis. A Vr of 3.5 or more and an MGV less than $50 \mathrm{~cm} / \mathrm{s}$ are the best predictive measures. Repair of correctable graft lesions with a Vr of 3.5 or more, or inflow, outflow, or graft lesions associated with an MGV less than $50 \mathrm{~cm} / \mathrm{s}$ are recommended.

\section{7 \\ Accuracy of Near Infrared Spectrophotometry (NIRS), in Monitoring Cerebral $\mathbf{S O}_{\mathbf{2}}$ During Carotid Surgery}

\section{A. Trani, A. Gasparre, B. Perretti, F. Bonanno \\ U.O.C. di Chirurgia Vascolare, Direttore: Dr. Francesco \\ Bonanno, Ospedale "Madonna delle Grazie", Azienda Sanitaria Locale di Matera}

Introduction: Aim of the study was the evaluation with Near Infrared Spectroscopy (NIRS) and neurological signs and symptoms of cerebral oxygen $\left(\mathrm{SO}_{2}\right)$ saturation during carotid clamping to perform endoarterectomy. To realize this registraztion we employed a cerebral oxymeter with two oxymetric electrodes (INVOS-400) that are necessary to obtain real values of cerebral $\mathrm{SO}_{2}$.

Materials and Methods: To realize this registraztion we employed a cerebral oxymeter with two oxymetric electrodes (INVOS-400) that are necessary to obtain real values of cerebral $\mathrm{SO}_{2} . \mathrm{So}_{2}$ was evaluated in 234 patients in II and III stage ASA, treated with open carotid endoarterectomy in general anesthesia. $\mathrm{SO}_{2}$ was monitored at first and third minutes after the carotid clamping.

Results: In 13 patients, test was associated with ischemic events and in these patients was employed Pruitt-Inahara shunt with complete restore of neurological state. We had no experience of cases with neurological stable deficits. $\mathrm{SO}_{2}$ rate was meanly of $18 \%+/-4.5 \%$ in patients that have required placement PruittInahara shunt and only of $7.5 \%+/-5 \%$ in which was not necessary his use. A decreas post-clamping that was superior to $15 \%-20 \%$ of pre-clamping $\mathrm{SO}_{2}$, was strongly correlated with clinically evident cerebral ischemic events.
Conclusions: Cerebral $\mathrm{SO}_{2}$ monitoring with Neared Infrared Spectroscopy (NIRS), is simple and non invasive method and in our experience, a decrease that exceeded $15 \%-20 \%$ was correlated with clinical manifestations pre and post intervention, with quickly and negative evolution and with very poor early-term outcome, In these cases, however, NIRS has allowed us monitoring in real time cerebral $\mathrm{SO}_{2}$ cerebral saturation and to complete the intervention with a complete resolution of focal or global neurologic deficits.

\section{8 \\ Biosynthetic Prosthesis, omniflow Type II, in Treatment of Severe Critical Lower Limb Ischemia: Preliminary and Medium Term Results, Our Experience and State of Art}

\author{
A. Trani, B. Perretti, A. Gasparre, F. Bonanno \\ U.O.C. di Chirurgia Vascolare, Direttore: Dr. Francesco \\ Bonanno, Ospedale "Madonna delle Grazie", Azienda \\ Sanitaria Locale di Matera
}

Introduction: The authors intend to evaluate the efficacy, in terms of biocompatibility, haemocompatibility and obviously of medium and long term patency of Omniflow type II prosthesis, composite material of ovin (sheep) connective tissue and a wowen polyester mesh, in manifacturing BK femoro-popliteal by-passes in alternative to venous material in patients with severe critical limb threatening ischemia of lower limbs.

Materials and Methods: Of twenty-one patients treated (21 by-passes), in which we have realized a femoro-popliteal by-pass under the knee (BK), men are 11 , women are 7 , with average age of 71 years: 11 were diabetics, 10 in II B stage, 5 in III stage, and 4 in IV stage A, and 2 in IV stage B. Follow-up was realized with color-duplex-scanning techique with instrumentation adeguately regulated (probe 7.5-14 Mhz PRF 3.5-5 Mhz, medium color gain) to 30 days, 3 months, 1 year, 18 months and 24 months.

Results: The patency rate was $100 \%$ to 30 days, $82 \%$ to 3 months, $77 \%$ to 1 year and $69 \%$ to 18 months, $57 \%$ to 24 months. There were no correspondence between tardive by-passes thromboses and limb-threatening, with an high rate of limb savage also in limbs with by-pass thromboses and poor distal run-off, observed with color-duplex-scanning and intraoperatively.

Conclusions: In the complex these results are acceptable and encouraging to prosecute the study. Successive and more analytic studies that evaluate to long term the difference in terms of patency, biocomaptibility and haemocompatibility are necessary and to estabilish definitively the prosthesis that is the real alternative to venous material in the cases in which this is no good to realize a BK femoro-popliteal or femoro-distal by-pass to treat a severe critical limb-threatening ischemia of lower limbs. 
289

\section{External Iliac Artery Posttraumatic Dissection Treated with Bypass in VSA. Case Report}

\author{
D. Trevisan ${ }^{1}$, P. Moglia ${ }^{2}$, D. Lovotti', P.G. Capretti' \\ R. Caronno \\ ${ }^{1}$ UO Chirurgia Vascolare, Ospedale S.Anna, Como, ${ }^{2} \mathrm{UO}$ \\ Chirurgia Generale e d'Urgenza, Ospedale S.Anna, Como
}

Introduction: External iliac artery injury from abdominal trauma is common $(0,4 \%)$. We describe a case of external iliac artery posttraumatic injury resulting in a artery dissection with lower limb acute ischemia.

Materials and Methods: An 19 years-old male sustained a severe penetranting injury in abdomen, with secondary dissection of the left external iliac artery. We treated an emergency surgical revascularization with a reversed long saphenous vein interposition graft.

Result: In the postoperative time we found an immediate revascularization of the lower limb, with complete resolution of the limb ischemia. For a week we assisted a revascularization syndrome, treated with medical therapy. Follow-up with angiogram (1 week), ecocolorDoppler (3 month) and clinical examination demonstrated that the patient returned at normal activities, with palpable distal pulses and ABI of 1.0.

Conclusion: The use of venous graft is indicated for: young age of patient; to reduce the risk of infection; to use a autologous materials; to reduce the risk of restenosis.

\section{0 \\ Endovascular Stenting for Common Iliac Artery Injury, Associated with Spinal Column Fracture. Case Report}

\section{Trevisan 1, G. De Nittis ${ }^{2}$, M. Galli², R. Caronno ${ }^{1}$ \\ ${ }^{1}$ UO Chirurgia Vascolare, Ospedale S.Anna, Como, ${ }^{2} \mathrm{UO}$ \\ Emodinamica, Ospedale S.Anna, Como}

Introduction: Outcomes of blunt iliac artery injures is associated with higer mortality, lower extremity amputation, compartment syndrome, and overall complications. Traditionally have been treated surgically via open reconstruction or direct repair. We present a case of acute common iliac artery injury following blunt abdominal trauma successfully managed with endovascular stenting.

Materials and Methods: A 31 years-old male was brought to the S. Anna Hospital in Como for a blunt abdominal trauma: dissection of right common iliac artery and spinal column fracture, show by angioTC scan. There was absence of right femoral pulses, with lower right limb ischemia. By bilateral femoral approach, a angiogram demonstrated the occlusion of right common artery for $6 \mathrm{~cm}$; at the same we recanalizzed the artery and placed across the occlusion a covered stent (Viabahn).
Result: Completion angiogram demonstrated good placement, widely patent stent, and good run-off. Immediately following the procedure, pulses was palpable. Follow-up with CT scan (3 month) and ecocolorDoppler (6 month) demonstrated adequate flow through the stented area, without evidence of migration. On clinical examination the patient was asymptomatic and was back to normal activities, with palpable distal pulses and an ABI of 1.0.

Conclusion: For emodinamic stable patient without a concomitant intra-abdominal injury requiring laparotomy, endovascular stenting is a valuable, feasible and effective surgery option.

\section{1 \\ Endovascular Treatment of Aortitis Caused by Brucella Melitensis}

M. Massara, F. Benedetto, S. Lentini, G. Vazzana,

G. DeCaridi, F. Spinelli

Cattedra e Scuola di Specializzazione in Chirurgia Vascolare-Università degli Studi Messina

Introduction: Brucellosis may be asymptomatic or produce a variety of clinical manifestations. Aortitis generally occurs as a secondary complication after endocarditis.

Case Report: A 81-year-old man was admitted in General Emergency for evaluation of fever, fatigue,abdominal pain and anemia. Abdominal computed tomography (CT) scan showed a rupture of aortic bifurcation. The patient was immediately conducted in operation room and he was submitted to placement of bifurcated endoprosthesis in abdominal aorta and iliac arteries. Blood exams showed an infection caused by Brucella Melitensis. Transthoracic echocardiogram, CT-brain and abdominal ultrasound exam excluded endocarditis or vegetations in these districts. A lombosacral MRI showed a L3-L4 lesion (spondylitis). The patient was treated with specific antibiotic therapy:doxycycline and rifampin. In postoperative day 7 CT scan showed an endoleak of I type in right iliac artery and the patient was submitted to placement of endoprosthesis in right iliac artery. After 5 days CT-scan showed the normal position of endoprosthesis and the presence of haematoma along psoas muscle; a CT-guided puncture of this blood collection excluded infection. The patient was discharged on postoperative day 35. After 12 months CT scan shows good position of endoprosthesis and no evidence of infection.

Discussion: Open surgery is the primary option in aortitis. Endovascular treatment is still controversial.

Conclusion: An early diagnosis associated with specific endovascular treatment and antibiotic therapy resolved this case. 


\section{2}

\section{Follow-up in Distal Revascularization: Survival and Limb Salvage}

F. Benedetto, F. Stilo, M. Massara, A. Modafferi, D. Barillà, S. Lentini, F. Spinelli

Cattedra e Scuola di Specializzazione in Chirurgia Vascolare-Università degli Studi Messina

Introduction: The aim of this study is to value survival and limb salvage after distal revascularization in patients affected by critical ischemia of lower extremities, through a retrospective analysis.

Materials and Methods: Retrospective review from Feb 2004 to Aug 2008 of 284 patients (M201, F83; mean age 70) suffering from critical limb ischemia (patients with extensive trophic lesions of lower extremities and pain at rest) and affected by common risk factors (diabetes mellitus $65 \%$; blood hypertension $62 \%$; smoke 42,2\%; dislipidemia 17,8\%; chronic renal failure $12,4 \%$; chronic renal failure in haemodialysis $12,4 \%$; BPCO20\%; miocardical ischemia $40 \%$; fibrillazione atriale $17 \%$ ).

Results: At the end of the period of follow-up of 5 years, completed on 279 patients $(98,24 \%)$, we recorded 188 survived patients $(68,4 \%)$ and 91 died patients (32,6\%). 170 survived patients $(90,5 \%)$ underwent to limb salvage and 18 patients $(9$, $5 \%)$ to amputation. 73 died patients $(80,2 \%)$ underwent to limb salvage and 18 patients $(19,8 \%)$ to amputation before death. In conclusion, the survival atlyear was $85,6 \%$, at 2 years $83,1 \%$, at 3years $74,3 \%$, at 4 years $62,1 \%$ and at 5 years $59,3 \%$. The limb salvage was $90 \%$ at 1 year, $89,7 \%$ at 2 years, $84,3 \%$ at 3 years, 82 , $9 \%$ at 4 years and $81,8 \%$ at 5 years.

Conclusions: Our results, according with international literature, suggests that distal revascularization avoids amputation in over $80 \%$ of survived patients and is associated to higher life expectation and a better quality of life.

\section{3}

\section{Lymphedema: Our Experience of Diagnosis and Therapy}

M. Agnati, G. Castrucci, L. Braccini, L. Traina, V. Gasbarro

U.O. Chirurgia Vascolare ed Endovascolare - Ferrara, Italia

Introduction: Lymphedemas are still today a hugely underestimated disease. This is mainly due to the fact that there is a serious lack of attention to the phenomena, a clear need for uniformity in its classification and above all, a scarcity of registered data.

There is at present, therefore an urgent need to create a global language and a database of epidemiological and significant statistics.

Materials and Methodology: Lymphedema diagnosis is based on the patients case history and specific targeted exams. These, together with first and second level diagnostics permit the respective definition of the morphological state of the hypodermis and the condition of both the lymphatic collectors and the lymph node stations.

Once the diagnostic aspect is defined, using the global classification system of CEAP-L, an appropriate therapeutic approach to the disease is then attainable.

Results: A multi-centric study, over a 5 year time period was conducted on 175 patients which permitted a diagnostical therapeutic database that is able to provide clear and structured therapy protocols for the pathology together with the realization of an IT database of lymphological clinical cases.

Conclusions: With clear and structured therapeutic protocols in place and an IT database of clinical cases to consult, the evaluation and quantification of the efficiency of various diagnostic and therapeutic approaches is now feasible.

It is also possible to create statistical research with regards to the different approaches of the various adhering centres to the project, thus realizing a network dedicated to the treatment of lymphatic pathologies.

294

\section{Fluid Dynamic of the Saphenofemoral Junction High Ligation Before and After Surgical or Endovascular Treatment}

\section{Boschetti, T. Rocca, V. Coscia, N. Cavallini, V. Gasbarro \\ U.O. Chirurgia Vascolare ed Endovascolare - Ferrara, Italia}

Introduction: Mathematical model research assimilated to the most frequent of clinical-anatomical situations of haemodynamics of the venous system, saphenofemoral high ligation incontinence and the conservation of the tributary veins pre and post surgical ablation treatment of the Great Saphenous Vein to maintain the physiological drainage of the groin area, thus reducing risk of recidivity and neovascularization. Such models may prove useful in the prediction of further evolution and the results of haemodynamic correction.

Materials and Methodology: Quantative and deterministic haemadynamic research of high ligation of the saphenofemoral junction utilizing haemodynamic measures by way of eco-colordoppler together with numerical reconstruction of local fluid dynamic parameters.

Results: Data Analysis permitted the detection of these elements:

1. In cases of deep system incontinence coupled with ostium incontinence, the course of the deep apparatus diverts towards the superficial apparatus thus causing hypertension.

2. In cases of saphenectomy (with radical crossectomy conservating the epigastric vein). A reflux flow is not observed as it is not counter-balanced by the hydrostatic column of the epigastric vein which acts as a drainage system.

3. In endovascular treatments with or without radical crossectomy the extension of the reflux flow on the remaining branch of the saphena is evident, when compared to the epigastric vein which does not demonstrate a directional flow from high to low.

Conclusions: The application of the models permit the objective evaluation of the haemodynamic condition of the 
Saphenofemoral high ligation and the evolution of the disease due to overloading pre and post treatment.

\section{5 \\ A Prospective Randomised Controlled Trial Comparing Foam Sclerotherapy Combined with Sapheno-Femoral Ligation to Surgical Treatment of Varicose Veins \\ R. Compagna, D. De Vito, R. Rossi, F. Fappiano, \\ L. De Magistris L. Sodano, M. Amato, B. Amato \\ Dipartimento di Chirurgia Generale, Geriatria, Oncologica e Tecnologie Avanzate, Direttore: Prof. G Persico, \\ Università degli Studi di Napoli Federico II}

Introduction: In this prospective randomised controlled trial we compare foam sclerotherapy combined with saphenofemoral ligation to surgical treatment of varicose vein. We want to evaluate the frequency of complications on the two arms of the trials.

Material and Method: 30 patients with primary varicose veins were randomly allocated in two groups:

GROUP A- in 15 patients we made ultrasound-guided sclerotherapy with sapheno-femoral ligation under local anaesthaesia.

GROUP B- 15 patients were underwent to sapheno-femoral ligation, stripping and multiple avulsions under spinal anaesthesia.

Results: At 4 weeks, there was no statistical difference in the complication rate between the two groups. At 6 months median CEAP class dropped from four pre-operatively to one following treatment in both groups. In group A 3 patients (15\%) had a recanalised vein which needed further sessions of foam sclerotherapy, resulting in a short-term closure rate of $75 \%$.

Conclusions: There were no statistical difference in the complication rate between the two groups.

\section{6}

\section{Association Between Recurrent Varicose Veins and Incompetentperforating Veins: Our Experience}

\section{D. de Vito, R. Compagna, L. Sodano, R. Rossi, F.Fappiano, B. Amato \\ Dpt. of General Surgery, University "Federico II" Naples, Chairman: Prof. Giovanni Persico}

Introduction: The aim was to investigate the association between the presence of incompetent perforating veins and development of recurrent varicose veins.

Methods: A consecutive group of patients presenting with varicose veins were examined using colour duplex ultrasonography. Pathological perforating veins were defined as those exhibit- ing bidirectional flow and a diameter of $4 \mathrm{~mm}$ or greater at the fascia.

Results: Between September 2008 and October 2009, 224 patients were examined. Primary varicose veins were found in 246 legs (165 patients) and recurrent varicose veins in 84 legs (59 patients). In patients with primary varicose veins, $108(44 \%)$ had incompetent perforators compared to $53(63 \%)$ of those with recurrent varicose veins. Also, for recurrent varicose veins, the percentage of patients with any given number of incompetent perforators was higher than for primary varicose veins. Overall, there was a higher number of incompetent perforators in those with recurrent veins compared to primary veins and this difference was significant at $95 \%$ confidence interval.

Discussion: Patients with recurrent varicose veins have both a higher prevalence and a greater number of incompetent perforating veins than patients with primary varicose veins.

297

\section{Treatment of Superficial Cutaneous Ulcers with Hyalosilver Spray ${ }^{\circledR}$, Update to January} 2010

\author{
L. Gazzabin*, M. Bucalossi*, A. Gazzabin*, F. Coratti, \\ R. Malatesti, L. Ceccaccio, F. Mariani* \\ Department of General Surgery, University of Siena, \\ Hospital "Santa Maria alle Scotte, ${ }^{*} T$ The Compression \\ Therapy study Group - (CTG)
}

Introduction: Hyalosilver ${ }^{\circledR}$ is an interactive dressing spray which contains Jaluronic Acid (0,2\%) and Colloidal Silver (2\%). The first component is a GAG of the extracellular matrix which is able to regulate the adhesion, growth and cell migration, improving the natural process of wound healing. The second component has antimicrobial and antibacterial properties acting on the respiratory processs of bacterial cell and preventing wound contamination.

Materials and Methods: The study is based on the treatment of superficial cutaneous ulcers, principally of the lower limbs. These ulcers was not complicated by critical infections and mostly due to venous insufficiency and traumatic accidents. Fiftyfour patients were treated until January 2010, thirty women and twenty-four men, mean age forty-eight years old. Over the spray we applied gauzes containing Jaluronic Acid.

Results: The combined use of these components provides a good control of exudate, promoting moist wound healing, cellular migration and a good control of bacterial contamination. Hyalosilver Spray ${ }^{\oplus}$ is easy to use, can be used by patients themselves and is not influenced by the conformation of the wound.

Conclusion: Hyalosilver Spray ${ }^{\oplus}$ is easy to apply and remove from the wound, can be put under compression stocking, gives a good control of exogenous bacterial cell and improves wound healing. According to our study this type of dressing is a good device in treating superificial cutaneous ulcers or other tipologies of them during re-epithelializating phase. 


\section{Day Surgery}

\section{8}

\section{Day Surgery and Resident in Surgery: A Feasible Situation?}

\author{
S. Agrusti, M. Canziani, V. Bertocchi, M. Cavalli, \\ G. Campanelli
}

University of Insubria, Varese (Italy), Dept. of Surgical Sciences Unit of General Surgery, Day and Week Surgery (Chief: Prof. G. Campanelli), Multimedica Hospital, Castellanza (va), Italy

Introduction: Day Surgery is a real possibility for residents to start their experience and to improve their anatomic knowledge and technical skills. The problem is how to give to the patients the maximum of safety and results for their Day Surgery.

Materials and Methods: In general surgery there are two principal procedures that could be an excellent "training". First one is the inguinal hernia repair by Lichtenstein technique: in this operation the regional anatomy has been well described and the techniques are well outlined and reproducible. However, it requires a solid knowledge of the necessary steps to complete the repair. Second procedure is laparoscopic cholecistectomy: very often, the resident in this kind of surgery don't have any idea of the anatomy in open field, but it is even true that "the new generation" of the young surgeons or resident is much more skilled to realize " virtual manoeuvre".

Results: The systematic review refer that there are no differences about surgeon activity in hernia repair with the supervision of a senior surgeon, except for the mean operative time.

About laparoscopic surgery is even more important to follow teaching criteria, because the possibility of a major complication is really much more elevated.

Conclusion: In presence of suitable teaching criteria the surgical outcome could be excellent even if operations are performed by young surgeons; supervision in those who are over the first steps, but have not completed their training has to be considered with attention, to avoid problems related to their self-assurance.

\section{9}

\section{Surgical Repair of Primary Lumbar Hernias}

G. Cavallaro, C. Paparelli, A. Polistena, G. D'Ermo, G. Orlando, R. Arena, G. De Toma

Dept. of Surgery "P. Valdoni”, Policlinico Umberto I, Sapienza University, Rome

Introduction: Primary lumbar hernias, rare hernia of the postero-lateral abdominal wall often representing the expression of a congenital defect, tipically arise in two areas of weakness, named superior triangle, and inferior triangle.
Clinical examination may be adjuvated by US or CT scan, that can reveal the abdominal wall defect with the hernia content (viscera or extraperitoneal fact).

Surgical repair has rapidly developed through recent years, similarly to the treatment of more frequent kinds of hernia (groin, epigastric), evolving form direct repair to mini-invasive techniques, even if, since the rarity of these hernias, precise knowledge of this complex anatomic region is required.

Materials and Methods: 15 patients (12 males, age 18-64, and 3 females, age 25-48) were submitted to surgery for primary lumbar hernias. 4 patients had bilateral lumbar hernias ( 3 bilateral Grynfelt's hernias, 1 bilateral Petit's hernia). No trauma or previous surgery history (neither lumbar nor abdominal) were reported.

Results: 12 patients underwent "open" access surgery, with mesh repair (polypropylene mesh in 10 cases, ePTFE dual-mesh in 2 cases). In 3 patients (two affected by bilateral hernia) laparoscopic transperitoneal approach was performed, with use of ePTFE dual-mesh, fixed to the abdominal wall by Pro-Tack devices. No drill or bone fixation was used.

Conclusions: Nowadays there are two valid alternatives in surgical repair of lumbar hernias. Open tension-free repair (with use of mesh), and mini-invasive repair. Both are safe and effective, even if smaller hernias can be even treated by open approach, with loco-regional anaesthesia and good cosmetic effect.

Larger hernias, or hernia with suspected viscera involvement, should require larger incision and viscera exploration. For this reason laparoscopic access would be preferable.

\section{0 \\ Biological Prostheses in Ventral Hernia Surgery: A Personal Experience \\ F. Ceci, F. Gossetti, M.A. Viarengo, L. D'Amore, P. Negro \\ "Sapienza" Università di Roma - Azienda Policlinico \\ Umberto I - Dipartimento di Chirurgia Generale "Paride \\ Stefanini" - U.O.C. Chirurgia Generale D e Day Surgery \\ (Prof. P. Negro)}

Introduction: The use of biological prostheses may represent an effective option to synthetic material in abdominal wall hernias repair in (potentially) contaminated fields. In these situations in fact the implant of synthetic meshes may lead to infection and consequent need to remove the prosthesis.

Materials and Methods: Our series consists in 29 implants of biological prostheses in complex abdominal wall hernia repair. The use of biological material has been justified by one of the following conditions: 1) synchronous gastrointestinal tract surgery; 2) patients treated with immunosuppressive drugs due to previous organ transplantation; 3) concomitant immunologic diseases; 4) treatment of parastomal hernias.

In all patients crosslinked porcine collagen meshes were implanted (18 Permacol, Covidien; 11 Collamend, Bard).

Results: At follow-up (min. 3 - max.54 months) one recurrence and one bulging were observed. Minor complications such as haematoma, seroma, wound infection were reported in 58\% of cases but only 3 patients needed a re-operation. 
Conclusion: Preliminary results seems to be satisfactory even if a longer follow-up to evaluate long-distance recurrences is needed.

\section{1 \\ Pneumatic Balloon Dilatation in the Treatment of Anal Fissures}

F.S. Mari, L. Masoni, R. Maglio, A. Milillo, A. Pancaldi,

F. Pindozzi, A. Dall'Oglio, G. Berardi, A. Brescia

UO Day Surgery, Azienda Ospedaliera Sant'Andrea, Università La Sapienza di Roma - II Facoltà di Medicina e Chirurgia

Anal fissure is one of the commonest perianal diseases, presenting with bleeding, itching, and severe pain. Internal anal sphincter spasm has been identified as the cause of pain and persistence of fissure. Internal Anal Sphincterotomy (IAS) has generally been considered as the standard operation for the treatment of anal fissure. The risk of fecal incontinence, bleeding and infection following IAS, has led to investigate the efficacy of non surgical procedures reducing the risks of permanent damage to the anal sphincters. Since 1993 we have performed over 700 pneumatic balloon dilations (PBD), by using a $40-\mathrm{mm}$ balloon inflated up to $1.2 \mathrm{~atm}$ and left in situ for five minutes under local anesthesia. We have reviewed the results in the last 250 cases. The chronic anal fissure became asymptomatic $1.3+/-2.2$ days after pneumatic balloon dilation and healed between the second and fifth week in 238 patients (95\%). Most failures were in patients who had previously undergone other treatments. We report only 9 recurrences after the procedure. In seventy-three cases pre and post-treatment anorectal manometry did not demonstrate faecal incontinence. Fortytwo patients underwent post PBD anal ultrasonography which did not show any significant sphincter defect. PBD is an non-invasive, effective, safe, easy procedure for the treatment of chronic anal fissures, with good clinical outcome.

\section{2 \\ Laparoscopic Cholecystectomy in Day Surgery \\ F.S Mari, L. Masoni, R. Maglio, A. Milillo, A. Pancaldi, F. Pindozzi, A. Dall'Oglio, G. Berardi, A. Brescia \\ UO Day Surgery, Azienda Ospedaliera Sant'Andrea, Università La Sapienza di Roma - II Facoltà di Medicina e Chirurgia}

Laparoscopic Cholecystectomy (LC) represents today the gold standard for the treatment of cholelithiasis. Despite the well known short post-operative course of laparoscopy in Italy today the mean hospital stay for LC is 2.6 days. Since november 2003 we have instituted in our Hospital a treatment protocol for cholelithiasis in Day Surgery. Our protocol includes patients up to
65 years of age, without major contraindications for the general anesthesia (ASA I or II) and no acute complications related to gall bladder stones. The surgical procedure usually starts at 8:00 am, we perform the LC with a 3 trocars technique with a mean duration of 40 minutes. After the intervention the patients come back to the ward and at $2 \mathrm{pm}$ they are allowed to eat a soup and walk. At 4 pm serum RBC, Bilirubin, Amylase and Lipase are checked and at $6 \mathrm{pm}$ they are discharged. From November 2003 to December 2009 we performed 150 LC: 46 men and 104 women. Mean age: 52 yrs (21- 65). Mean operative time 55 minutes (29- 85) with no open conversions. Patients were discharged 8-10 hours after the procedure. Five patients needed one night hospitalization, two for postoperative pain and three for PONV. The ambulatory LC is justificated by semplification of the procedures, reduction of hospitalization costs and also by a low impact in patients.

\section{3 \\ Stapled Haemorrhoidopexy in Day Surgery: Our Experience}

F.S. Mari, L. Masoni, R. Maglio, A. Milillo, A. Pancaldi, F. Pindozzi, A. Dall'Oglio, G. Berardi, A. Brescia

Azienda Ospedaliera Sant'Andrea, Università La Sapienza di Roma, II Facoltà di Medicina E Chirurgia

Haemorrhoidal disease is one of the most widespread diseases, considering that $50 \%$ of subjects above the age of 50 years is affected by this pathology. In 1998 A. Longol proposed a new surgical technique for the approach to haemorroidal prolapse consisting in the correction of mucous prolapse using a circular stapler. From January 2005 to December 2009 we performed at our Day Surgery Unit 316 stapled haemorrhoidopexy (SH). Of this series, 22 patients needed an overnight hospitalization: 13 for pain and postoperative nausea and 9 for urinary retention. In 9 (2.8\%) cases, patients were readmitted for postoperative bleeding and treated with revision of staple line haemostasis. In our experience, $285(90.2 \%)$ patients were treated and discharged 8-10 hours after the surgery successfully. The main postoperative complications were: pain and tenesmus for over 2 weeks in 11 cases, up to 2 weeks in 21 cases, infection of staple line in 6 cases, incontinence to gases in 2 patients treated with pelvic floor rehabilitation protocol. The patients selection is the key to successful ambulatory $\mathrm{SH}$, for this reason the improvement of the result is related to how the inclusion criteria are strictly respected. Our experience shows that the $\mathrm{SH}$ can be safety performed in day-care setting if the structure organization allows to identify and treat any early or late postoperative complication. 
304

\section{New Original Technique to Reduce the Haemorrhage Rate of Stapled Haemorroidopexy in Day Surgery}

F.S. Mari, L. Masoni, R. Maglio, A. Milillo, A. Pancaldi, F. Pindozzi, A. Dall'Oglio, G. Berardi, A. Brescia

UO Day Surgery, Azienda Ospedaliera Sant'Andrea, Università La Sapienza di Roma - II Facoltà di Medicina e Chirurgia

In the last century many surgical interventions were proposed to treat haemorrhoidal disease. In 1998 Antonio Longo introduced the stapled haemorroidopexy ( $\mathrm{SH}$ ) and today, in some hospitals, this procedure is performed in day surgery unit. Postoperative bleeding represents the major constraint to perform this procedure in ambulatory setting. The incidence of postoperative bleeding is from 1.5 to $13.3 \%$. In approximately $40 \%$ of cases it is necessary to place some haemostatic stitches to manage the intraoperative anastomotic bleeding. Since 2004 we have routinely performed SH in Day Surgery and we have tried some techniques to reduce the intra and postoperative bleeding rate. In the 2009 we tried to use a bioabsorbable staple-line reinforcement (BSR) and to evaluate its efficacy and safety we enrolled, between September and December 2009, 70 patients with symptomatic, II-III degree haemorrhoidal disease. The patients were randomly divided in two groups with using or non using the BSR performing the SH. The results of our study show, in BSR group, a statistically relevant reduction of intraoperative bleeding, of the need of additional anastomotic stitches and of postoperative pain. The study also demonstrate that the use of BSR allows to reduce the postoperative bleeding rate and to perform this procedure safely in the day surgery unit.

\section{Emergency Surgery}

305

WITHDRAWN:

Perforated Peptic Ulcer: A Ten Years Surgical Experience

This abstract is withdrawn.
306

Abdominal Dressing with VAC: From Wound Management to Open Laparostomy

E. Giorgini, S. Di Saverio, G. Senatore,

N. Clemente N. Antonacci, A. Biscardi, S. Villani,

F. Baldoni, G. Tugnoli

Department of Emergency and Trauma Surgery, Maggiore Hospital Bologna.

Introduction: The principles of VAC wound management were introduced in the 90s and applied to the open abdomen. The mechanism of macro-micro deformation allows exudate management, maintenance of fascial edges domain, protection of abdominal contents from intra-extra peritoneal fluids to reduce edema and accelerate the healing.

Materials and Methods: Our experience includes: damage control laparostomy for trauma, severe acute pancreatitis (SAP), peritonitis, frozen abdomen and entero-cutaneous fistula. GranuFoam dressing was used for entero-cutaneous fistula, Vacuum Pack was performed in tree cases of open laparostomy: trauma, SAP, peritonitis. The other patients were treated with the ABThera ${ }^{\mathrm{T}}$ NPT System.

Results: Entero-cuteneous fistula healed after two applications. Open laparostomy after trauma was performed due to abdominal hypertension but was removed after 24 hours for bleeding. The first case SAP required surgical decompression after necrosectomy; the abdomen was closed after two cycles. In the second patient, ABThera ${ }^{m M}$ NPT System was used eight times draining 23 litres overall. Two cases of peritonitis from ileal and colonic perforation were treated respectively five and two times with resolution of the infected exudate and maintenance of wall. The Abdomen frozen was closed with biological mesh after tree cycles of VAC. No complication VAC-related were observed.

Conclusion: VAC dressing is a flexible system usefull to resolve clinical situations where Total Abdomen Closure is not possible or contra-indicated. The ABThera ${ }^{\mathrm{m}} \mathrm{NPT}$ is most effec- 
tive for removing fluids and infected materials as well as reducing edema.

307

WITHDRAWN:

Prophylactic Appendectomy in Acute Abdomen: Is It a Worthwhile Procedure?

This abstract is withdrawn.
Methods: 100 patients (49 male) with presumed appendicitis were randomized: group 1 (n. 58) was treated following Alvarado score: $\geq 7$ surgery, 4-6 further diagnostic tests with delayed surgery or discharge, $\leq 3$ discharged. Group 2 was treated on the basis of an experienced surgeon evaluation and free application of diagnostic tests.

Results: $63.8 \%$ of group 1 and $62 \%$ of group 2 had surgery. In group $1,58.62 \%$ had early surgery, $20 \%$ clinical observation, $5.17 \%$ delayed surgery and $36.2 \%$ were discharged. In group 2 , $57 \%$ had early surgery, $21.4 \%$ clinical observation, $4.76 \%$ delayed surgery and $38 \%$ were discharged. After surgery appendicitis was confirmed in $91.9 \%$ of group 1 and $100 \%$ of group 2. Alvarado score $\geq 7$ had $83 \%$ sensitivity, $68 \%$ specificity, $77 \%$ PPV, $79 \%$ NPV. Experienced surgeon evaluation showed a 100\% sensitivity, 100\% specificity. Overall diagnostic tests executed in patient population were 83 in group 1 (1.43/patient) and 57 (1.35) in group 2.

Conclusions: Alvarado score was developed as an objective criterion for diagnosis of appendicitis. This study observed that Alvarado score had lower sensitivity and specificity than experienced surgeon evaluation and induced the execution of more exams per patient. These results suggest that surgeon supervision is useful in evaluation and treatment of appendicitis.

\section{9 \\ Splenic Trauma: The Case of a Young Woman with Splenic Trauma Treated Conservatively

\author{
L. Simonelli, B. Tarantino, T. Ricciardulli, V. Silvestri, \\ C. Modini \\ Dipartimento Emergenza Accettazione Umberto I Roma
}

Nowadays the treatment of polytrauma patients underwent a significant change due to innovations that have been in the field of diagnostic instrument. These innovations have led to the development of new therapeutic strategies for making the multidisciplinary management. Therefore, the choice of treatment needs careful planning in management of patient, in accordance with modern linesguides that provide an immediate identification and stabilization of most serious injuries and the achievement of a "permissive hypotension" or "Controlled hypotension" to reduce blood loss that may affect mortality. An important role is played by the interventional radiology if capable and resourceful in many cases can save the life of patient without necessarily surgical intervention. The case before us, we want to talk on a young patient came at our emergency room because of an accident in who had multiple rib fractures, fracture of left clavicle, and splenic laceration with hematoma peri-sigmoid leakage of contrast medium on CT abdomen. The patient was underwent arteriography with embolization thus obtaining operational control of 1 1.bleeding without surgery and less hospital days.

Introduction: A modified Alvarado score, ranging 1-10, has been compared with clinical evaluation by an experienced surgeon in the diagnosis of acute appendicitis. The score was based on migration of pain to right-lower-quadrant (RLQ), anorexia, nausea/vomiting, rebound pain, tenderness in RLQ, WBC count, C-reactive protein, temperature. 
310

\section{MRI Findings of Intestinal Ascariasis in a Primary Malignant Lymphoma and Small Bowel Occlusion. Case Report}

\author{
F. Guerra, E. Sacco, D. Crocetti, F. Velluti, C. Modini
}

Dipartimento Emergenza-urgenza, Policlinico Umberto I, "sapienza", Università Di Roma

Introduction: Ascariasis is the most common worm infection in man. Mostly spread through tropical and sub-tropical areas, in association with poor health conditions, this infection is unusual in industrialized countries where, a large percentage of affected people are immigrants from developing countries.

Intestinal ascariasis is usually detected as an incidental finding. During the intestinal phase of the disease, the adult worms usually remain clinically silent, sometimes causing a variety of nonspecific abdominal symptoms. Bowel obstruction is a frequent complication that can be moreover complicated by intussusception, perforation, and gangrene of the bowel.

Materials and Methods: We report a case of a 30-year-old male presented to the emergency department with the symptoms of a bowel obstruction due to an ascariasis in a patient with primary malignant lymphoma of the small bowel.

Results: Ascariasis was described by MRI, an enterotomy and a resection of a stenosing mass of the distal ileum was performed.

Conclusions: In Ascariasis MRI can be helpful to diagnose a surgical emergency. Concurrent bowel obstruction due to ascaris lumbricoides infestation, in a patient with malignant lymphoma of the small bowel: coincidence or consequence?

\section{1}

Changes in the Outcomes of Severe Trauma Patients: A Western European Experience in Trauma ICU (1996-2010)

\section{E. Giorgini, S. Di Saverio, G. Aimone, F. Cancellieri, \\ G. Senatore, N. Clemente, N. Antonacci, S. Villani, \\ A. Biscardi, F. Baldoni, G. Gordini, G. Tugnoli \\ Maggiore Hospital Trauma Center}

Introduction: Recent safety regulations for RTA prevention, aimed to decrease mortality for trauma. We evaluated their effect during the last 15-years period.

Methods: 60247 trauma admissions (2183 deaths) were recorded (1996-2010). 2935 trauma patients (ISS >16) were admitted to TICU and recorded in prospectively collected database. $97.1 \%$ were blunt trauma. A retrospective review of outcomes stratified for year was carried out; all available factors were included in multivariate analysis of prognostic value.

Results: Overall mortality was $17.2 \%$, Respiratory Morbidity was $23.3 \%$. A significant increase of trauma admissions occurred (before/after 2001, p<0.01). Mean GCS (10.2) increased (Test Trend $\mathrm{p}<0.05)$. Mean age (44.12), ISS (24.83), mechanism didn't change significantly whereas mortality decreased showing two marked drops, from $25.8 \%$ in 1996 , to $18.3 \%$ in 2000 and again down to $10.3 \%$ in 2004 (Test Trend $\mathrm{p}<0.01$ ). TBI accounted for $58.4 \%$ of the causes of death, Hemorrhagic shock in $28.5 \%$ and MOF/Sepsis in $13.1 \%$. However TBI-related decreased with raise of MOF/Sepsis (Test Trend $\mathrm{p}<0.05$ ). Significant predictors of mortality were year of admission $(\mathrm{p}<0.05)$, age, hemorrhagic shock and SBP, ISS and GCS and $\mathrm{pH}, \mathrm{BE}($ all $\mathrm{p}<0.01$ ). In the subgroup underwent emergency surgery the same factors remained significant, plus Blood-Units transfused $(\mathrm{p}<0.05)$. Surgical time (mean 71 min, showing significant trend to reduction) wasn't significantly associated with mortality $(\mathrm{p}=0.06)$.

Conclusions: Mortality of severe trauma decreased significantly during the last 15 years as well as mean GCS whereas ISS remained stable. New safety regulations influenced incidence and severity of TBI and seemed to improve the outcomes. ISS seems better predictor of outcome than RTS No disclosure.

\section{Endocrine Surgery}

\section{2 \\ Intraoperatory Neuromonitoring of Recurrent Laryngeal Nerves in Thyroid Surgery \\ G. Dionigi, F. Frattini, M. Annoni, P. Castano, A. Leotta, F. Rovera, S. Rausei, L. Boni, R. Dionigi \\ Centro di Ricerche in Endocrinochirurgia, Università degli studi dell'Insubria - Varese}

Introduction: Aim of this study is to describe our personal experience in thyroid surgery using intraoperative neuromonitoring (IONM) of recurrent laryngeal nerves (RLN).

Methods: Four-hundred and thirty patients for a total of 966 nerves at risk were analysed. IONM of RLN was performed using the monitoring system by Medtronic with an endotracheal tube. The standard procedure consists of monitoring laryngeal and vagal nerves before and after each thyroid lobectomy. All patients underwent pre- and post-operative laringoscopy.

Results: IONM was performed in $98 \%$ of patients. In the first series of 100 procedures system failure was observed in $25 \%$ of cases (malposition of the tube was the first cause); this rate decreased to $5 \%$ for the following patients. The vagal nerve and the RLN were tested and monitored successfully in all cases. Accurate visual identification of RLN was possible in $76 \%$ of cases. The rate of transient RLN palsy was $2.6 \%$. No permanent RLN palsy was registered. Accuracy of IONM in detecting RLN dysfunction was $97 \%$. When a negative electromyographic signal showed an impaired RLN function, a two-stage thyroidectomy was planned.

Conclusions: This is the first series of thyroidectomies performed with IONM in Italy. This study revealed that IONM is a useful and reliable tool for identification of RLN and evaluation of 
RLN function. The accuracy of this procedure is strictly correlated to the experience of the surgeron and the anaesthesist.

313

Intraoperative Neuromonitoring of Recurrent Laryngeal Nerves in Thyroid Surgery: Cost Analysis

G. Dionigi, F. Frattini, M. Annoni, P. Castano, A. Leotta, F. Rovera, S. Rausei, L. Boni, R. Dionigi

Centro di Ricerche in Endocrinochirurgia, Università degli

Studi dell'Insubria - Varese

Introduction: Introperative neuromonitoring (IONM) of recurrent laryngeal nerve (RLN) improves identification and preservation of RLN. Nowadays data about economic impact of IONM are not available yet. Aim of this study is to evaluate cost of thyroidectomy performed with IONM.

Methods: Analysis of the costs was performed in an Italian University Hospital. In the study were included costs related to personal, operative theatre, materials, drugs, and hospital stay. Three settings were evaluated: conventional thyroidectomy, thyroidectomy with IONM (by Medtronic) in an high-volume centre ( $>275$ procedures per year), thyroidectomy with IONM in a lowvolume centre ( $<90$ procedures per year)

Results: Costs for a thyroidectomy with IONM were between euros 3713 to 3770 (setting 2 and 3, respectively), 7-9\% higher than costs in setting 1 (euros 3471). Most relevant costs were related to materials (25\%), operative theatre (16\%), and personal (14\%). The costs associated with surgical procedure accounted for the $58 \%$ of the whole hospitalization and the IONM related costs only represented the $7 \%$ of the thyroidectomy weight.

Conclusions: IONM system in thyroid surgery provides a minimal impact on health financial resources as it only represents $7 \%$ of general costs.

\section{4}

\section{Minimally Invasive Videoassisted Selected Lateral-Cervical Lymphectomy: 2 Case Reports}

\section{Marfisi, D. Bettini, A. Zaccaroni}

U.O.S. Chirurgia Endocrina, Ospedale Morgagni-

Pierantoni, Forlì

Introduction: In the last years endoscopic approaches have been proposed for the treatment of thyroid disease; minimally invasive videoassisted (VA) thyroidectomy according to Miccoli's technique has been recognized to be the most successfully and performed.

Traditional open approach is reserved to lateral cervical (LC) dissection; at present none has been described with central VA approach.
Materials and Methods: We report our first experience with minimally invasive VA-LC neck lymphectomy with a median 3-4 cm cervicotomy.

Patient 1: female with hyperparathyroidism and high serum level of calcitonin suspicious for medullary carcinoma confirmed at histology,. It was performed a total thyroidectomy, single parathyroidectomy, bilateral central and LC selective lymphectomy (II-III-IV level).

Patient 2: female with high serum thyroglobulin, thyroid metastatic central-LC disease (III and IV level).

Results: Patient 1: 18 lymph nodes have been excised ( 1 metastatic of 6 of the central compartment, 5 of the II and III and 2 belonging to the IV level all reactive).

Patient 2: 17 lymphatic structures sampled ( 5 metastatic of 6 of the central compartment, 1 metastatic of 3 of III level and 8 negative of IV).

No complication occurred (hypocalcemia, nerve deficit, haemorrhage).

Postoperative pain was minimized.

Surgical operating time was $240 \mathrm{~min}$ for the $1^{\text {st }}$ and 130 for the $2^{\text {nd }}$ case like open technique.

Serum calcitonin and thyroglobulin normalized after surgery.

One year follow up is disease-free.

Conclusion: In selected cases minimally invasive VA-LC dissection can be feasible and performed. In our experience this technique has produced a good oncologic result, acceptable surgical time without complications.

315

WITHDRAWN:

The Role of Intraoperative Cytology and Frozen Section Examinations in the Decision-Making of 231 Thyroid Fnacytological Presentations

This abstract is withdrawn. 


\section{7 \\ Bilateral Papillary Thyroid Cancer in Patien Exposed to Depleted Uranium: a Really Correlation? \\ C. Folliero, E. Bonaiuto, M.A. Lerose, A. Rizzuto, N. Innaro, \\ G. Vescio, R. Sacco \\ U.O. Chirugia Generale, Università degli Studi Magna \\ Graecia, Catanzaro, Direttore:prof R. Sacco}

\section{6 \\ Cystic Parathyroid Lesion: Two Cases Report and Discussion of the Literature}

\author{
P. Princi, M. Guerriero, S. Berardi, M.P. Ridolfini, P Caprino, \\ F. Palumbo, F. Pacelli \\ Centro di Ricerca, Assistenza e Formazione ad Alta \\ Tecnologia nelle Scienze Biomediche "Giovanni Paolo II", \\ Università Cattolica del Sacro Cuore - Campobasso
}

Objective: Parathyroid cyst are rarely encountered in neck surgery and often misdiagnosed. We review the case incidence of parathyroid cystic lesions among all neck procedure.

Methods: Two cases of parathyroid cystic lesion were recognized and included in the study. The medical records of these patients were reviewed with special attention to pre-operative diagnosis.

Results: Two female patients were referred to our center because of neck swallowing, dysphagia, choking and occasional dysphonia. Ultrasound imaging revealed neck masses of 66x44mm and $44 \times 25 \times 23 \mathrm{~mm}$ respectively. Serum i-PTh was $238 \mathrm{ng} / \mathrm{ml}$ and $699 \mathrm{ng} / \mathrm{ml}$ respectively, however sestamibi scintiscan was negative in both. Intra-cystic fluid evaluation of PTH showed elevated value (more than $5000 \mathrm{ng} / \mathrm{ml}$ ). The patients underwent to a parathyroidectomy combined with thyroid lobectomy and total thyroidectomy, respectively. Intra-operative PTH decreased till normal value and serum calcium levels become normal post-operatively. Final histology showed benign parathyroid cystic adenoma and concomitant benign thyroid disease.

Conclusion: Degeneration of parathyroid cyst is a rare entity and sometimes represent a diagnostic challenge. Pre-operative US and sestamibi evaluation showed low diagnostic accuracy. The role of needle aspiration with fluid parathyroid assay is fundamental.
Introduction: Papillary cancer (PTC) is the most common t5hyroid's malignant tumor with an incidence of $60-70 \%$. In $60-85 \%$ ha a focal presentation while in $5-10 \%$ unknown at the first diagnostic approach appears in the controlateral lobe. The gold standard treatment is total thyroidectomy with law recidive 's and mortality's rates. The postoperative radioiodine didin't improve surgical results. The choose of surgery is strictly connected with patient's medical history and surgeon's experience.

Case Report: A man 31 years previously operated of right lobe istmectomy for a suspected nodule after depleted uranium exposure in the Balkans. On the evidence of histological results "Well differentiated PTC extensive to perithyroid tissue" underwent to new surgical approach on left lobe. The histologycal result confirm the diagnostic suspect of PTC also in left side.

Discussion: The exposure to depleted uranium, above all among Gulf War or Balkans military seems correlated to higher Incidence of PTC, sometimes also synchronous with Leukemia, lymphoma or testicular cancer. Long term's follow up and randomizied studies are still necessary for the evaluation of bio molecular PTC correlation, still demonstrated in vitro.

Conclusion: As high cancer risk PTC needs an aggressive surgical approach. The Reoperation after subtotal thyrodectomy or lobectomy is justified and validated. A careful 1 year follow up is necessary.

\section{8 \\ Comparison of Three Different Methods of Haemostasis During Total Thyroidectomy: A Prospective Randomized Controlled Study}

\section{Ruggiero, G. Lissidini, A. Gurrado, E. Poli, G. Piccinni, M. Testini \\ Department of Applications in Surgery of Innovative Technologies. University Medical School of Bari, Italy}

Introduction: The employment of safer and more effective haemostatic procedures in thyroid surgery is very useful to avoid blind surgical manoeuvres causing morbidity. The study compares the efficiency of different haemostatic devices: traditional haemostatic surgical instruments (THIS) vs Tabotamp vs FloSeal agents.

Materials and Methods: Between January 2005 and December 2007, 155 consecutive patients underwent total thyroidectomy. Patients with Graves' and hyperfunctioning thyroid diseases, cervico-mediastinal goitres, disorders of haemostasis and coagulation, and those who had undergone lymphadenectomy or 
MIVAT were excluded from the study. The patients were randomized in group A $\left(n=49\right.$, THIS); group B $\left.(n=52 \text {, Tabotamp })^{\circ}\right)$ and group $\mathrm{C}(\mathrm{n}=54$, FloSeal $)$.

Results: Mean operating time was reduced in the FloSeal group $(105 \mathrm{~min})$ vs $\mathrm{A}(133 \mathrm{~min}, p=0.02)$ and $v s \mathrm{~B}(122 \mathrm{~min}$, $p=0.0003$ ). Drain removal occurred earlier with FloSeal $(\mathrm{p}=0.006$ vs THIS; $p=0.008$ s Tabotam $p^{\circ}$ ) resulting in shorter postoperative hospital stay in the FloSeal group ( $p=0.02$ vs THIS; $p=0.002$ vs Tabotamp ${ }^{\circ}$ ). No significant differences in terms of postoperative morbidity among the groups were observed.

Conclusion: In our study, the use of FloSeal was associated with a significant reduction in operating time, mean time of drainage stay and postoperative hospitalization.

\section{ENT}

\author{
319 \\ Sclerosing Polycystic Adenosis of the \\ Parotid Gland: A Case Report \\ P. Canzi ${ }^{*}$, A. De Vito*, G. D'Agostino*, A. Rencricca*, \\ F. Padovani**, A. Campanini*, C. Vicini* \\ *Department of Special Surgery, ENT and Oral Surgery \\ Unit, Ospedale Morgagni Pierantoni, - University of Pavia \\ in Forlì, Italy, ${ }^{* *}$ Department of Pathology, Ospedale \\ Morgagni Pierantoni - Forlì, Italy
}

Introduction: Salivary gland tumors account for a relative small but quite heterogeneous population of head and neck neoplasm. Sclerosing polycystic adenosis (SPCA) is an extremely rare lesion of uncertain nature with histological similarity to fibrocystic disease of the breast. Frequently misdiagnosed as various types of salivary gland carcinoma, we report a case of SPCA occurring in a young patient undiagnosed until histopathologic confirmation.

Material and Methods: A 26 year-old female was referred to our attention for the presence of a gradually enlarging mass in the region of the right parotid gland. After complete ENT examination including imaging study, the patient was submitted to trucut of the lesion. Morphological analysis demonstrated ductal infiltrating carcinoma of the parotid gland. Total parotidiectomy was performed with a complete preservation of the facial nerve.

Results: Histological examination on surgical specimen demonstrated SPCA of the right parotid gland. No facial nerve deficit was reported.

Conclusions: SPCA is an extremely rare lesion of very difficult histological diagnosis and despite the few cases reported in literature, it is needed a careful awareness of this entity.

\section{0}

\section{Transoral Robotic Surgery in Pediatric Obstructive Sleep Apnea: A Case Report}

P. Canzi , F. Montevecchi*, G. Tenti ${ }^{*}$, I. Dallan ${ }^{*}$, C. Vicini ${ }^{*}$

*Department of Special Surgery, ENT and Oral Surgery Unit, Ospedale Morgagni Pierantoni, - University of Pavia in Forlì, Italy, ${ }^{* *}$ ENT Unit, Azienda Ospedaliera Universitaria Pisana, Italy

Introduction: The incidence of OSAHS in children is estimated to be $2 \%$ and treatment should be carefully tailored to the airway obstructive causes. Adenotonsillectomy has been proved efficacy only in $83 \%$ of patients. When correctly identified, Base of the Tongue (BOT) hypertrophy must be considered a not exceptional sleep related obstructive condition. On our preliminary experience, robotic techniques revealed good tolerability in tongue base hypertrophic OSAHS patients. No previously reported in literature, we describe the first case of paediatric OSAHS submitted to BOT resection by means of Transoral Robotic Surgery (TORS).

Material and Methods: A 16 year-old male was referred to our attention for Severe OSAHS related primarily to BOT hypertrophy. In addition to clinical, endoscopic and imaging findings, pre- and post-operative polysomnographic data were collected and analysed.

Results: TORS procedure was completed successfully removing $50 \mathrm{cc}$ of BOT tissue. No complications has been observed. A liquid diet was allowed from the $1^{\text {st }}$ day and length of hospital stay was 9 days. A complete recovery from OSAHS has been achieved.

Conclusions: Minimally invasive techniques are not sufficient to manage satisfactorily huge tongue base hypertrophy. When properly selected, TORS proved to be a feasible and effective approach to BOT hypertrophy also in paediatric OSAHS patients.

\section{1 \\ Low Power $\mathrm{CO}_{2}$ Laser for Treatment of Bilateral Vocal Chord Paralysis}

G. Motta, A. Lecce, G. Marcuccio, A. Brancaccio, D. Testa

Second University of Study of Naples

Introduction: The bilateral adduction paralysis of the vocal chords produce a mechanical respiratory obstruction of the upper respiratory tract by reduction of glottic space and consequently increase in breathing resistance.

We wanted to detect the effectiveness of a new system of $\mathrm{CO} 2$ laser treatment for this disease and the results at a distance by considering the development of complications.

Materials and Methods: 8 patients were selected - 5 females $(62.5 \%)$ and 3 males $(37.5 \%)$ with a mean age of 43.9 years, with adduction vocal chords paralysis treated with aritenoidectomy using a CO2 laser, to last generation, used in "superpulse" modality at low power (3.5-4 Watt). Patients were evaluated by means of fibrolaryngoscopy, FEV 1, FEV max, Multi Speech 3500 (Kay) spectrography, aerodynamic study with Aerophon II, for three 
years after the treatment, every 3 months during the first year and then every 6 months.

Results: The results showed that the variation technique induce a low rate of complications due to the poor production of fibrin in the operative field and a slight charring of tissue, with a more rapid functional restore in breathing and in making vocal use.

Discussion: Endoscopic surgery using a CO2 laser is an excellent choise to traditional surgery because of his advantages and reduced traumatism.

Conclusion: The bilateral adduction paralysis of the vocal chords have to be treated with aritenoidectomy using CO2 laser used in "superpulse" modality at low power (3.5-4 Watt).

\section{2}

\section{Intra-operative Neuromonitoring and Neurostimulation of the Recurrent Laryngeal Nerve in Thyroid Surgery: A Randomized Clinical Trial}

\section{I.J. Fernandez, O. Piccin, A. Marcantoni, O. Cavicchi \\ U.o. Otorinolaringoiatria, Ospedale Sant'orsola - Malpighi, Azienda Ospedaliero-universitaria Di Bologna}

Introduction: Intra-operative functional testing of the nerve is gaining a wide diffusion, particularly neurostimulation (NS) and introperative neuromonitoring (IONM). The impact of these methods on avoiding recurrent laringeal nerve (RLN) injury is still controversial. The aim of our study was to compare the efficacy of RLN direct stimulation alone to direct stimulation in association to intra-operative neuromonitoring.

Materials and Methods: The study was designed as a randomized clinical trial. Between January 2008 and April 2009 were included 250 patients (455 nerve at risk) randomized into 2 groups: control group of 125 patients (229 nerves at risk) assigned to NS with palpatory feedback, and the study group comprising 125 patients (229 nerves at risk) assigned to IONM and NS. The prevalence of RLN palsy and accuracy of the feedback signals were compared between the groups.

Results: The overall RLN injury incidence for nerve at risk was $2.7 \%$ in the study group and $2.6 \%$ in the control group. No statistically significant difference was found between the groups. Either palpatory feedback or IONM feed back to RLN direct neurostimulation obtained an elevated negative predictive value (respectively $98.2 \%$ and $99 \%$ ) and a poor positive predictive value (26.7\% and $22.2 \%)$. The accuracy of the two methods resulted to be similar (respectively $95.8 \%$ and $92.7 \%$ ).

Conclusions: The IONM, compared to NS alone, did not significantly reduce the incidence of nerve injury after thyroid surgery. Furthermore the two methods showed a similar accuracy in predicting postoperative paralysis.

\section{3}

\section{Reconstruction of the Oral Cavity After Oncologic Surgery: Anatomical and Functional Aspects}

\author{
V. Damiani ${ }^{1}$, A. Rocco' ${ }^{1}$ B. Pesucci², G. Montemari3, \\ G. Bellocchi \\ ${ }^{1}$ ENT Department, San Camillo-Forlanini Hospital, Italy, \\ ${ }^{2}$ Maxillo-facial surgery Department, San Camillo-Forlanini \\ Hospital, Italy, ${ }^{3}$ Plastic surgery Department, San Camillo- \\ Forlanini Hospital, Italy
}

Objective: The reconstructions of the oral cavity are designed to reproduce the original structures to allow a resumption of feeding and phonation functions to which this area is deputies. The choice between different methods is conditioned by the entity, the form and function of the section, considering the need of bone or soft tissue reconstruction or the filler purposes of reconstruction.

Methods: The Authors present some cases of patients affected by oral cavity cancer submitted to surgical demolition and reconstruction with free microvascular flaps. Specifically, flaps used were: anterolateral tight flap, radial forearm free flap, rectus adbominis free flap and fibula free flap.

Results: Bearing in mind the division of the oral cavity in two functional areas, one set of tissues with high motility and one set of tissue with low motility, and evaluating the specific characteristic of the four flaps we used, in term of availability of donor soft tissue or bone, of reliability of vascularization and of possibility of following implants, we obtained optimal hestetic and functional results in all patients.

Conclusion: In our experience, the use of just four free flaps allows to cover any reconstructive needs after demolitive surgery of oral cavity.

\section{4 \\ Treatment of Mandible Fractures: A Personal Experience}

L. Calzolaro, E. Molini, F. Longari, M. Gullà, A. Frenguelli

Clinic Otolaryngology of Perugia

Introduction: The mandible is the most common site of facial trauma. The diagnosis is clinical-radiological and the treatment is almost always surgical in order to restore a functioning mandible, correct occlusion.

Methods and Materials: We examined 50 patients who were treated in our clinic for mandible fractures from January, 2006 to December, 2009. There were 41 men and 9 women, 18 to 82 years old, with unifocal fractures in 37 cases and multifocal fractures in 13 . The areas which involved the fractures were as follows: parasymphysis $20(32 \%)$, angle $19(30 \%)$, condyle 7 (11\%), symphysis $6(9 \%)$, region of the body $6(9 \%)$, ramus $3(5 \%)$, and the alveolar process $2(3 \%)$. The complications were classified by Alert et al. 1999 by 4 types: during treatment (open wound infec- 
tion), as a consequence of inappropriate treatment (bad occlusion), surgical mistakes, and lack of treatment (bad occlusion).

Results: 50 patients were treated, where 13 had closed surgical treatment (intermaxillary fixation), 13 had open treatment (reduction with plates), and 24 had combined treatment. The most frequent areas of fractures in our study were the parasymphysis.

Conclusion: According to the literature the most frequent complications of mandibular fractures is infection, bad occlusion, pseudarthrosis, ankylosis and paresthesia. In our case, the only complications were an altered habitual dental occlusion in two cases of multifocal fractures treated with reduction with plates.

\section{5}

\section{Congenital Nasal Pyriform Aperture Stenosis: Report of Three Cases}

\section{Berlucchi, B. Pedruzzi, M. Sessa \\ Department of Pediatric Otorhinolaryngology, Spedali Civili, Brescia, Italy}

Introduction: Congenital nasal pyriform aperture stenosis (CNPAS) is an uncommon disorder causing nasal obstruction in neonates. This disease can cause important breathing and feeding problems. At imaging, a width of the pyriform aperture less $11 \mathrm{~mm}$ in a term infant is considered diagnostic. CNPAS may be associated with abnormal dentition, modified palate shape, endocrinal, and neurological anomalies. Conservative or surgical treatment is in relation to severity of symptomatology and extent of lesion. We report 3 neonates with CNPAS and describe diagnostic procedures and therapy of this unusual pathology.

Materials and Methods: Three infants were admitted for nasal obstruction associated with episodic apnea, rib retraction, and feeding troubles in 1 case. Two patients were male and 1 female, with a mean age of 60 days. All patients underwent CT-sinuses and MR-brain. Conservative treatments (i.e., nasal washing, nasal aspirations, nasal topical vasoconstrictor, or temporary nasal stent) and surgical management via sublabial approach were performed in 2 children and 1, respectively.

Results: CT-scan showed a CNPAS in all patients. Central neurological system anomalies were present in 1 child, whereas dental abnormalities were observed in another. To date (mean follow-up: 48 months) all children are symptom-free.

Conclusions: CNPAS is a rare disease of pediatric age. CT of sinuses is mandatory to obtain a correct diagnosis. A conservative treatment can be performed when the width of the pyriform aperture is more of $5 \mathrm{~mm}$. In other cases, a surgical treatment is required.

\section{6 \\ Endoscopic Diode-Laser Treatment for Acquired Localized Tracheal Stenosis in Children}

M. Berlucchi, B. Pedruzzi, M. Sessa

Department of Pediatric Otorhinolaryngology, Spedali

Civili, Brescia, Italy

Introduction: Acquired localized tracheal stenoses, which have a lower frequency than generalized one, have shown an increased incidence in last decades. This is due to an higher survival of premature baby underwent prolonged tracheal intubation and/or tracheostomy. The authors describes 2 cases of infants affected by acquired localized tracheal stenosis successfully treated by endoscopic management.

Materials and Methods: 20- and 5-month-old girls were admitted to Department of Pediatric Otorhinolaryngology, Spedali Civili, Brescia, Italy for severe dyspnea. Both children underwent previously heart surgical treatment followed by prolonged orotracheal intubation. A rigid laryngotrachealscopy (RLTS) was performed in both cases and an endoscopic tracheal treatment by diode-laser was carried out. Then, the patients remained oro-tracheal intubated for 48 hours. A follow-up endoscopic examination was performed.

Results: At RLTS performed at admission a circumferential tracheal scar tissue stenosis was observed. This disorder caused a tracheal space decrease of about $60 \%$ in one patient and $80 \%$ in other case. At the removal of oro-tracheal tube performed after surgical treatment, both children breathed normally. The followup tracheal endoscopy showed a stabilized tracheal correction.

Conclusions: Acquired localized tracheal stenosis is an increased disease. Endoscopic treatment by diode-laser is a conservative and reproducible procedure and may be considered a valid alternative to traditional tracheal surgery. A long-standing experience in RLTS is mandatory to obtain good results.

\section{7 \\ Extended Laterocervical Abscesses and Descending Necrotizing Mediastinitis: Treatment and Outcome}

\section{Simone, I. Sinopoli, E. Bianco, C. Viti, A. Camaioni \\ ${ }^{1}$ Università degli studi di Foggia, ${ }^{2}$ Azienda Ospedaliera San Giovanni - Addolorata di Roma}

Introduction: Descending necrotizing mediastinitis (DNM) represents a rare, nearly fatal, pathology, often caused from polymicrobial deep neck abscesses.

If a DNM is suspected, a CT scanning is mandatory. According to radiological parameters, DNM can be classified as type I (limited to the upper mediastinal space), type IIA (extended to the lower-anterior mediastinum) and type IIB (extended to both anterior and posterior lower mediastinum).

In this study we examine 8 patients and analyse literature. 
Materials and Methods: During 5 years, 8 patients with extended laterocervical abscess, complicated by DNM in 4 cases, were treated surgically at our ENT Departement; mean age 52 years (range 23-76). In 50\% of cases an hypopharynx/esophagus fistula was the cause of abscess, while in the remaining cases was always a previous peritonsillar abscess.

Results: All patients underwent surgical drainage with only cervical approach. In two cases a review of surgical wound was necessary for fistulas. 3 patients needed more surgical drainage to achieve complete healing. All patients were discharged without major sequelae.

Conclusion: In literature, reported mortality rates in DNM range from $25 \%$ to $40 \%$ and it didn't vary in last years despite innovations in critical care and surgical techniques. Early diagnosis is the exclusive factor influencing outcome. A surgical drainage and debridement of neck and mediastinum are required.

In agreement with main authors, the DNM with type I involvement can be effectively treated by trans-cervical approach only. In contrast, in patients with type IIA involvement is useful a mediastinotomy or thoracotomy. Finally, in patients with type IIB involvement thoracotomy is necessary.

\section{8 \\ New Technology in ENT Surgery: The Cliniporator}

\author{
M. Simone1, C. Viti², A. Camaioni ${ }^{2}$ \\ ${ }^{1}$ Università degli studi di Foggia, ${ }^{2}$ Azienda Ospedaliera San \\ Giovanni - Addolorata di Roma
}

Introduction: The Cliniporator is a new technology that uses electrical impulses to obtain a cellular poration. This allows to entrap into the cell cytotoxic drugs otherwise not permeable to the cell membrane (electrochemotherapy).

Exclusive validated indications for electrochemotherapy (ESOPE project, 2005) are skin tumors.

This study valuate feasibility and efficacy of a new approach: intraoperative using of Cliniporator to sterilize surgical margins and to treat surgically inaccessible areas.

Materials and Methods: We used Cliniporator in 3 cases: an oropharyngeal recurrence carcinoma, an extended oro-hypopharyngeal carcinoma and a laterocervical metastasis from not head and neck cancer.

We used bleomicin intravenously and applied the electrical stimulation through needle electrodes. Special sanitary measures isn't necessary to use drug or Cliniporator.

Results: In the days following the application we noticed an extensive layer of fibrin throughout the treated area, with re-epithelialization of region in 4 weeks. Oral intake occurred in media in 2 weeks (range 5 days- 4 weeks).

Currently two cases are disease free, even if the follow-up is short and one died by disease.

Conclusion: This new treatment for cancers of the head and neck is very interesting, but needs further study with larger series and longer follow-up to be able to evaluate the real benefits.

\section{9 \\ Follow-up of Middle-ear Cholesteatoma Surgery: Canal Wall Up Versus Canal Wall Down Tympanoplasty}

\section{A. Ranieri, N. Bianco, V. Ranieri ${ }^{1}$, M.W. Rossetti}

Functional Unity of Otorhinolaryngology, S. Rita Clinic Atripalda, Italy, ${ }^{1}$ E.N.T. Clinic, Second University of Naples, Italy

Introduction: The ideal goals of cholesteatoma surgery should be the permanent removal of disease and the preservation of good hearing function.

There are no universally accepted opinions about the choise of surgical technique and autcome of surgery for cholesteatoma in different age group and localization.

Materials and Methods: In this study, we presented the results of 92 patients (49 males, 43 females; mean age 32 years; range 7 to 56 years) who underwent functional surgical techniques for cholesteatoma between January 2001 and January 2006. An open technique was prefered in $72 \%$ of the cases. All the patients were followed-up for at last three years.

Results: The incidence values for the recurrent cholesteatoma in closed tympanoplasty and in cases of open techinque were $18 \%$ and $9 \%$ respectively. In all cases it was evidenced the positive effect of the stapes superstructure on hearing results.

Conclusion: The age the patients and localization of cholesteatoma are very important factors than determinate the type of surgical procedure and the results of surgery for middle ear cholesteatoma. A closed technique is better for younger children and for attic and sinus cholesteatoma, while in all other cases, an open technique seems more appropriate. Open technique is associated with very law incidences of residual or recurrent cholesteatoma in the majority of patients. The presence of stapes superstructure positively affects hearing status indipendently for the technique used.

\section{0 \\ Results and Complication of the Baha System}

\section{Gullà, M. Faralli, L. Malafronte, G. Ricci, A. Frenguelli \\ Clinic Otolaryngology of Perugia}

Introduction: The aim of this work is to re-examine our experience with the treatment of conductive and mixed hearing loss using the Baha system.

Materials and Methods: The system was implanted in 47 patients (16 adults and 31 children under the age of 14), bilaterally in two cases. The causes of hypoacusis were bilateral congenital aural atresia (31 patients), bilateral chronic otitis media or outcomes of middle-ear surgery (9), and otosclerosis (7). The following parameters were assessed: mean preoperative air and bone conduction thresholds for the frequencies of 500, 1000, 2000 and $4000 \mathrm{~Hz}$; mean postoperative threshold with the Baha; hearing 
improvement calculated by subtracting the postoperative threshold with the Baha from the preoperative threshold for air conduction in the better ear; speech audiometry test; improvement in the quality of life, calculated using the Glasgow Benefit Inventory for the adult patients and the Glasgow Children's Benefit Inventory for paediatric patients; frequency and type of surgical complications. Follow-up ranged from 6 to 38 months.

Results: The audiological results were satisfactory, with air-bone gap closure in $85.1 \%$ of cases. In terms of quality of life, assessment using the GBI showed a clear-cut improvement in health for all the 45 patients that answered to the questionnaires in our study. Out of the 49 operations that were performed, complications were reported in 3 cases (6.1\%): 2 cases of skin regrowth around the titanium screw and one in which the abutment was not osseointegrated.

Conclusions: The data from this study show that the Baha system offers a high percentage of success, which can significantly improve the patient's quality of life, and a low rate of complications.

331

Hypopharynx Reconstruction with Pectoralis Major Myofascial Flap: Our Experience on Fourty-five Patients

\section{Damiani', S. Galla', A. Rocco', G. Montemari², G. Bellocchi ${ }^{1}$ \\ ${ }^{1}$ ENT Department, San Camillo-Forlanini Hospital, Italy, ${ }^{2}$ Plastic surgery Department, San Camillo-Forlanini Hospital, Italy}

Background: The pectoralis major myofascial flap (PMMF) is a simple variant of the pectoralis major myocutaneous flap (PMMC) and allows to avoid some of the disadvantages of the Ariyan's technique.

Methods: This is a retrospective analysis of 45 hypopharyngeal reconstructions (40 immediate reconstructions after subtotal pharyngolaryngectomy and 5 performed during additional revision surgery) using PMMF flap, performed from February 1995 to February 2008 in the Department of Otolaryngology at the S. Camillo Hospital in Rome, in cooperation with the Department of Plastic Surgery.

Results: In our series, a total of 3 postoperative flap-related complications were identified with an overall complications rate of $6.7 \%$. The incidence of major flap complications requiring surgical revision was of $2.2 \%$. Two minor complications were recorded: an hypopharyngeal stenosis and a salivary fistula, both managed nonoperatively. Total or partial necrosis did not occur in any case and the preoperative goal of the PMMF flap procedure was met in 44 of 45 (98\%) cases.

Conclusion: The PMMF flap procedure is a safe one-step procedure, that is particularly useful for partial hypopharyngeal recontructions, allowing to obtain a high overall success rate without some of the limitations of the PMMC and fasciocutaneous free flaps.

\section{2 \\ Pre- and Intraoperative Evaluation of Glottic Lesions in the Planning of Excisional Biopsy; Personal Experience in 142 Patients}

\author{
M. Giudice, E. Digilio, F. Ferreli, P. Castelnuovo
}

Department of Otorhinolaryngology, University of Insubria, Ospedale di Circolo e Fondazione Macchi Varese

Introduction: Endoscopic clinical appearance of glottic leuko-eritroplakias are non-specific for final diagnosis. Only the pathologic evaluation of the specimen is able to the definitive diagnosis. We present the validity of our work-up using laryngostroboscopy (LS) and intraoperative hydrodissection with saline infusion (HDR) into Reinke's space before an "en bloc" resection procedure.

Methods: 142 patients with vocal cord leuko-eritroplakias observed between January '03 and December ' 08 underwent to preoperative examination with laryngostroboscopy (LS) and intraoperative work-up employing rigid endoscopes and hydrodissection (HDR) into Reinke's space, in order to appraise the potential deep invasion of the vocal cord layered structure.

Results: After work-up results, we performed CO2 laser cordectomies, according to the European Laryngological Society (ELS) Classification: Type I cordectomy (subepithelial) in 83 Patients, Type II (subligamental) in 30, Type III (transmuscular) in 29 respectively. Histopathological diagnoses demonstrated keratosis without atypia in 47 patients, keratosis with mild atypia in 18 , moderate atypia in 13 , severe dysplasia in 13 , myoblastoma in 1 , microinvasive carcinoma, invasive carcinoma in 49 , fibromiosarcoma in 1.

Discussion: Unilateral glottic leuko-eritroplakias of the vocal cord may be treated with excisional biopsy alone. Lesions ranging from keratosis without atypia to invasive carcinoma without infiltration of the vocal muscle can be safely treated by Type I (subepithelial) or Type II (subligamental) cordectomy. In order to get the best compromise between oncological radicality and preservation of the layered structure of the vocal cord, in our Institution we routinely apply a pre- and intraoperative diagnostic work-up including tests previously described in the literature.

333

\section{Oncological and Functional Results in Head and Neck Reconstructive Surgery}

\author{
V. Conz, S. Savini, E. Checcoli, F. Morolli, C. Bianchini \\ Clinica Orl Università Degli Studi Ferrara
}

Background: Our aim was to evaluate postoperative quality of life, oncological and functional results in a group of patients with cancer of the oral cavity and oropharynx treated with head and neck reconstructive surgery.

Methods: We reviewed the records of 63 patients who underwent to resection and reconstruction for advanced squamous cell cancer from 1989 to 2009. 
We considered risk factors, complications, overall survival (OS) and disease free survival (DFS). Functional results were evaluated means of endoscopy and clinical-logopedic protocol. For psychological study we used the questionnaire EORTEC QLQ 30-35.

Results: The main identified risk factors were: smoking, alcohol and their association.

Thirty-four (53\%) patients presented an advanced stage at diagnosis. OS at 5 years was $49 \% ; 41 \%$ of patients relapsed within 2 years. The main negative predictor factors were: the locoregional extension and the lymph node metastases.

Four patients, presenting postoperative suffering flap needed revision on anastomosis and in 2 cases there was flap necrosis.

19 patients $(30,15 \%)$ had positive margins while 28 patients (44\%) underwent postoperative radiochemotherapy.

Conclusion: The advanced stage and lymph node metastases at diagnosis adversely affect the OS and DFS. Swallowing function and intelligibility of speech are more compromised in patients treated for oropharyngeal cancer compared to patients with carcinoma of the oral cavity. The advanced stage, mandibular surgery and postoperative radiotherapy show negative influence on the functional outcomes.

Psychological study showed that intervention had a negative impact mainly on individual relationships with the outside world.

\section{4 \\ Hypopharyngeal Reconstruction with ALT in Oncologic Surgery: Our Experience}

Dr. Sinopoli

Introduction: The reconstruction of hypopharyngeal defects is challenging for plastic reconstructive surgeons. The anterolateral thigh flap (ALT), has recently gained popularity.

In this study we exposed our experience in hypopharyngeal reconstruction with ALT.

Materials and Methods: In 20 months, 8 patients underwent to an extended demolitive surgery involving hypopharynx with revascularized ALT free flap reconstruction in our Institute.

On 3 patients the primary lesion started in hypopharynx, 4 patients showed an oropharyngeal lesion after involving hypopharynx and the last a laryngeal lesion that involved medial wall of hypopharynx. The lesions was all carcinomas . All the patients underwent to a selective neck dissection too.

Results: There were not operative or peri-operative deaths. The mean hospital stay was 35 days. The follow-up was 15 months.

There were no major complications during or after surgeries. In two cases we had minor donor site complications.

Currently 4 patients tolerate a regular diet, the remaining 3 a pureed one.

Postoperative radiotherapy was applied to two case. Two patients showed intraoral recurrences, treated with new ablative surgery alone and currently are disease free. One patient died by locoregional recurrence after one year.

Currently the patients of this study, except the deceased, are disease free.
Conclusion: In our experience the hypopharyngeal defect extended to oropharynx and/or larynx can be safely replaced with ALT free flap obtaining good functional and aesthetic results without major complications.

\section{5 \\ Submandibular Gland's Adenoid Cystic Carcinoma: Case Report and Review of the Literature}

G. Marini, C. Caporale*, A. Martelli, M. Costa , M. Fusetti

'Cattedra di Otorinolaringoiatria, Università degli Studi, L'Aquila, " U.O. di Otorinolaringoiatria, Ospedale Civile dello Spirito Santo, Pescara

Introduction: Salivary gland tumors represent between $2 \%$ and 6.5\%, approximately, of all head and neck tumors. Adenoid cystic carcinoma (ACC) is a very rare epithelioid tumor with different prognostic factors relating to overall survival, late distant metastases and local recurrences. The median age is 61 years with a male predominance.

Case Report: A 45-years-old healthy woman was referred to our clinic with a painless mass appeared in the right submandibular region 6 month ago. Physical examination showed a painless, hard, mobile on superficial and deep plane, round mass. The patient underwent CT scan which showed right submandibular gland increased in size $(3 \times 3 \mathrm{~cm})$, with development in its midanterior portion. She underwent total right sialoadenectomy. The final pathology report showed adenoid cystic carcinoma of submandibular gland with mixed (tubular and cribriform) growth pattern; focal infiltration of perineural tissue was found. The patient underwent omolateral functional neck dissection; all lymph nodes were free of disease (T3, N0, M0). Patient started radiation therapy after executing CT-PET exam.

Conclusion: According to the literature ACC is a rare malignant neoplasm. The failure of antibiotic and anti-inflammatory therapy in sialoadenitis may suggest the possibility of malignancy. Surgical excision with postoperative radiation offers a better disease-free survival. Given the possibility of late relapses it should be recommended a long-term and careful follow-up. 


\section{Experimental Surgery}

336

\section{A Semiautomatic Guide System for Interventional Radiology Procedures}

\author{
E. Faiella, G. Luppi, M. Nezzo, G. Cazzato, R.F. Grasso, \\ B.B. Zobel
}

The aim of the study was to assess the clinical benefit of a robotic system to perform procedures of interventional radiology on lung (SIRIO). The system, by an infrared sensor, can indicate patient and needle position and their movements in the space. SIRIO doesn't use ionizing radiation. A prospective study in double blind of 90 patients underwent to lung biopsy was performed. The cutaneous sensor was placed on each patient. We obtained the $100 \%$ of technical success, that is gaining the lesion. The $t$ Student test was performed. A p value less than 0.05 was interpreted as statistically significant. As for as the average of lesion gaining time, it was 14(+/-7)minutes for SIRIO vs 25(+/-12)minutes for traditional biopsies. For these data the $\mathrm{p}$ value was less than $0.05(0.0451)$. The average of scans number was 3.4(+/-1.5) for SIRIO, vs $6(+/-4)$ for traditional method. As for as the average dose to gain the lesion, in DLP it was $256.25(+/-149)$ for SIRIO, vs for the traditional biopsy is $446(+/-433)$, while if calculated in $\mathrm{mAs}$ it was respectively of $1617(+/-1043)$ and $2835(+/-3108)$. About the pointing error in the first needle piling, calculated as divergence angle between the ideal path and the real one, the average error was $9^{\circ}$ for SIRIO vs $24^{\circ}$ for traditional biopsy. Thus we can assert that SIRIO resulted very reliable to perform biopsies on the lung.

\section{Gynecological Surgery}

337

Correction of Genital Prolapse with Tensionfree Vaginal Mesh Kit Prolift: Our Experience

C. Formisano, M. Scala, V. Gargano, F. Formicola, C. Di Carlo, G.A. Tommaselli, C. Nappi

Department of Obstetrics and Gynecology - University of Naples Federico II - Naples - Italy

Introduction: Aim of this study was to evaluate the efficacy and safety of Prolift vaginal mesh kit in the management of vaginal wall prolapse.

Materials and Methods: Thirty-six women undergone Prolift procedure were studied. Pelvic organ prolapse was classi- fied according to the PoP-Q system. After surgery, history, physical examination with speculum exam, vaginal palpation and PoP-Q scoring were repeated at 6 weeks, 3, 6 months and 12 months. Failure was defined as recurrent prolapse or any symptomatic prolapse. Operative time, estimated blood loss, intra- and post-operative complications and hospitalization stay were recorded.

Results: We performed 18 cystocele corrections, 6 rectocele corrections, 8 associated procedures and 4 vaginal vault prolapset. Operative times were $22.1 \pm 5.6$ (anterior Prolift), $19.3 \pm 3.2$ (posterior Prolift) $46.9 \pm 10.1(\mathrm{~A}+\mathrm{P})$ and $51.4 \pm 15.5$ minutes (total Prolift). Twenty-two patients (61.1\%) underwent concomitant surgical procedure for SUI. No intraoperative complication was recorded. Estimated blood loss was higher in the total Prolift group. Hospitalization stay was one day for all patients, with the exception of two (deep hematoma and febrile morbility). Complications were a deep hematoma of the obturator fossa with urinary retention, 2 cases of urinary retention, and one abscess formation and mesh exposure that needed drainage of the abscess and resection of the mesh. We did not observe and recurrence or failure of Prolift and one case of persistent SUI. All patients had an improved anatomy of the pelvis.

Conclusions: Prolift tranvaginal tension-free mesh system for the correction of pelvic organ prolapse seem to be effective and safe if used by trained and experienced surgeon.

\section{8 \\ Modified Total Laparoscopic Hysterectomy for the Treatment of Very Enlarged Uteri: Technical Aspects and Review of the Literature \\ G. Roviglione ${ }^{1,2}$, M. Ceccaroni $i^{1,2}$ \\ ${ }^{1}$ Gynecologic Oncology Division, International School of Surgical Anatomy, Sacred Heart Hospital, Negrar, Verona, Italy, ${ }^{2}$ Department of Obstetrics and Gynecology, European Gynaecology Endoscopy School, Sacred Heart Hospital, Negrar, Verona, Italy}

Introduction: Fibromatosis may cause anomalous bleeding, uterine enlargement and rarely anomalous growth of the uterus, for which the traditional surgical treatment has been abdominal hysterectomy. However, development of minimally invasive techniques has led to major safeness of the laparoscopic route.

Materials and Methods: We report a case of total laparoscopic hysterectomy performed on a uterus weighing more than $3,000 \mathrm{~g}$ and some considerations about surgical techniques and indications.

Results: A 48-years-old nulliparous woman underwent total laparoscopic hysterectomy with preservation of the adnexa. The operation was conducted following some technical adaptation to traditional technique. A laparoscopic morcellation of the uterus was then performed using a laparoscopic cold-blade scalpel. Vaginal extraction of the uterine fragments was not possible due to substenosis, thus they were manually extracted by an ultralow mini-laparotomic access. Total blood loss was $<100 \mathrm{ml}$, whole surgical time was $300 \mathrm{~min}$ with $90 \mathrm{~min}$ needed for hysterectomy. The 
dry weight of the uterus was $3030 \mathrm{~g}$. The patient had an uneventful postoperative stay and was discharged from hospital 3 days after surgery. At one-month follow up the patient reported no complication after discharge and a prompt recovery.

Conclusions: With expert hands in laparoscopic surgery and with proper technique and instrumentation, TLH can be performed successfully in case of very enlarged uteri, with no increase in complication rates and short-term recovery comparable with that in women with moderately enlarged uteri. Thus, uterine size and shape and uterine weight should not represent a limit to this kind of approach.

\section{9}

\section{Preliminary Results of Systematic Robotic Para-Aortic Lymph Nodes Dissection in Patients with Gynaecologic Malignancies: Technique and Surgical Outcomes in an Italian Referral Cancer Center}

F. Sanguineti, M. Peiretti, V. Zanagnolo, L. Bocciolone, G. Aletti, F. Landoni, A. Maggioni

Division of Gynecology, European Institute of Oncology, Via Ripamonti 435, Milan, Italy.

Introduction: With this study we would like to evaluate the technique and the surgical outcomes of robotic transperitoneal infrarenal aortic lymphadenectomy.

Methods: In a single institution 31 patients underwent robotic transperitoneal infrarenal aortic lymphadenectomy performed by 4 different surgeons as staging of gynaecologic malignancies: we described the technique and we analyzed the results retrospectively.

Results: Thirty-one patients with a median age of 41 years (range, 19-61). The BMI ranged between 20 and $26.5 \mathrm{Kg} / \mathrm{m} 2$. The median operating time was 257 minutes (range, 174- 390 minutes), the median docking time was 4.5 minutes, while the median table rotation time (from completion of pelvic surgery and undocking to completion of table rotation) was 11 minutes. The median number of para-aortic nodes removed was 14 (range, 2 - 31). There was only 1 conversion to laparotomy due to the operative findings of more advanced disease.

Conclusions: Robotic transperitoneal infrarenal aortic lymphadenectomy can be performed adequately and safely with the robotic column at the patient's head. Operating table rotation and additional trocar sites are needed when used in conjunction with robotic pelvic surgery.
340

\section{Outcome and Quality of Life in Women Treated with Nerve-Sparing Radical Hysterectomy for Cervical Cancer: A Comparative Study}
E. Spagnolo', M. Ceccaroni2,3, P. Casadio', F. Bruni2,3, G. Pontrelli2,3, M.R. Talamo ${ }^{1}$, L. Minelli ${ }^{3}$, G. Pelusi ${ }^{1}$
${ }^{1}$ Department of Gynecology and Obstetrics, Bologna University Hospital, Bologna, Italy, ${ }^{2}$ Gynecologic Oncology Division, "Sacred Heart Hospital", Negrar, Verona-
Italy, ${ }^{3}$ Department of Gynecology and Obstetrics, European Gynaecology Endoscopy School, "Sacred Heart Hospital" Negrar, Verona, Italy

Introduction: Radical Hysterectomy (RH) (type III) represents the gold standard for IA2-IIA cervical cancer. The purpose of this study was to compare Nerve Sparing Radical Hysterectomy (NSRH) and Classic Radical Hysterectomy (CRH) about: clinic outcome, follow up, quality of life.

Methods: From January 1997 to September 2008, 50 patients, IA1-IIIB, were enrolled in this study. We measured post-voiding residual and self-catheterization rates at discharge. All patients underwent clinical-instrumental follow-up, survival analysis and a quality of life (sexual,bladder,bowel-rectal) questionnaire.

Results: CRH was performed in 24 patients (group 1):19 (group 1a) underwent laparotomy and 5 (group 1b) laparoscopy. NSRH was performed in 26 patients (group 2): 16 (group 2a) underwent laparotomy, 10 (group 2b) laparoscopy. We showed that patients of Group 2 (compared with group 1) represented: lesser self-catheterization rates at the time of discharge $(\mathrm{p}=0.07)$, higher rate of adequate $(<100)$ post-voiding residual after 18 months $(p=0.03)$, better quality of life $(p<0.05)$. Survival analysis showed that nerve-sparing technique was performed with the same oncological radicality of the classic one.

Conclusions: Nerve-sparing technique revealed itself as a safe procedure, with an adequate oncological radicality and a lower rate of dysfunctions. A sudden identification of fibers and proper surgical landmarks consents to reduce bladder, bowel and sexual impairment. 
Maxillofacial Surgery

\section{1}

\section{Mandibular Swelling: A Rare Case of Langerhans Cell Histiocytosis in an Adult}

\author{
P. Biondi, E. Sarti, S. Stea \\ Maxillo-Facial Surgery Unit, Villa Maria Cecilia Hospital, \\ Cotignola (RA), Italy
}

Introduction: We report a case of histiocytosis involving mandible and femoral neck in an adult. The differential diagnosis and treatment plan in adult disease is stressed.

Materials and Method: A 43 years old man presented with a swelling extending from the midline to the left side of the mandible. This patient reported a spontaneous femoral neck fracture one year before. Oral and femoral biopsy was performed. The diagnosis in both localizations was Langerhans cell histiocytosis. The patient underwent surgical treatment of the mandible and of the femoral neck, followed by chemotherapy for six months.

Results: The follow-up at 24 months revealed no clinical or radiological signs of relapse. The computerized tomography showed a good bone mineralization of both mandible and femoral neck.

Conclusion: Langerhans cell histiocytosis is a rare disease, especially in adults. Oral disease presented with soft tissue ulcers, periodontal damage, non-healing extraction socket. Differential diagnosis includes keratocystic odontogenic tumor, ameloblastoma, cyst (odontogenic cist, lateral periodontal cyst, botryoid cyst), central giant cell lesion. The Histiocyte Society is contemplating recommending multiagent chemotherapy for bone-only disease. In this case we preferred a surgical treatment before the chemotherapy to improve the quality of life of the patient and to reduce the relapse.

\section{2 \\ Double Warthin of Parotid Gland: Case Report and Literature Review}

G. Nicolai, B. Lore, P. Buttà, I. Madanas, D. Fortellizze, L. Calabrese

Università degli Studi di Roma «Tor Vergata», Cattedra di Chirurgia Oro-Maxillo-Facciale, Scuola di Specializzazione in Chirurgia Odontostomatologica, Direttore Prof. Leonardo Calabrese

Introduction: Warthin's tumor or papillary cystadenoma lymphomatosum accounts for $10 \%$ of salivary gland tumors. It affects mainly males (forth-seventh decade) and occurs more frequently in the parotid gland. It is thought to develop from the epithelial cells restant within the intraparotid lymph nodes (remains of Neisse-Nicholson) on heterotopic salivary gland. Smoking is a risk factor for bilateral Warthin's tumours. Macroscopically, it presents as an indolent, slow growing, delimited, often floating mass. Microscopically it consists of epithelial and lymphoid tissue. The lesion is benign and surgical removal is therapeutic. Multiple tumours of salivary glands are rare. Two categories may be distinguished: with identical or different histology. In either group the tumous can be unilateral or bilateral, synchronous or metachronous.

Materials and Methods: A 50 year-old male presented to our departement showing a painless hard movable swelling covered by normally coloured skin, arising from eight months in the left parotid region. The patient underwent total parotidectomy with removal of both tumors identified on palpation and CT with and without contrast, preserving the facial nerve.

Results: The histological examination deposed for "Dual Warthin's Tumor of the parotid gland".

Discussion: The tumor with the more frequent multicentric development and uni/bilateral involvement is Warthin's tumour. In a study conducted in 1989 on 25 patients with synchronous tumors of unilateral salivary glands were detected only 2 cases of synchronous tumors: a Warthin tumor with a " mixed tumour"; and 2 parotid Warthin's tumors. The case considered represents one of the few synchronous parotid Warthin's tumours present in literature.

\section{3}

\section{One Step Oral Rehabilitation}

\section{F. Gallo ${ }^{1}$, P. Biondi ${ }^{2}$}

${ }^{1}$ Istituto Ortopedico Galeazzi, reparto di chirurgia MaxilloFacciale, Milano, ${ }^{2}$ Villa Maria Cecilia Hospital, reparto di chirurgia Maxillo-Facciale, Cotignola (Ra)

Introduction: The surgical procedure suggested, offers the ability to rehabilitate the smile and, on a wider scale the aesthetics of the middle third and inferior facial with only one surgery articulated in a fracture according to Le Fort I combined with an inlay bone connection and an immediate charge implatology.

Materials and Methods: Since 2005, 5o cases of severe jaw bone atrophy have been treated with the method of ONE STEP ORAL REHABILITATION. The surgery proposed, even if it's articulated in more than one passage, (osteotomy according to Le Fort I, inlay connections, immediate charge implatology) aims to give, with one surgery both, the function of the jawbone and the aesthetics to the middle third and inferior facial.

Results: The cases that have been treated demonstrate the efficiency of the technique that, even though complex, offers the surgeon, and consequently the patient, the possibility to rehabilitate the smile with attentive programming but with a single surgery.

Conclusions: Even though this represents a technique that requires experience, due to the numerous variables, before, during and after surgery, that may occur, the ONE STEP ORAL REHABILITATION technique offers noticeable advantages for the surgeon's ability to reduce surgical time and for its predictable aimed at results. 
344

\section{Endoscopic Removal of Supernumerary Tooth from the Nasal Cavity}

M. Clementini, A. Morlupi, C. Agrestini, P. Bollero,

A. Barlattani

Dipartimento di Scienze Odontostomatologiche, Università Tor Vergata, Roma

Introduction: A case report showing a removal of a supernumerary tooth from the nasal cavity by means of an endoscopic approach is presented.

Materials and methods: A 9-year-old healthy child presented to our department because of the right central incisor which appeared clinically rotated. The observation of orthopantomography revealed the presence of a supernumerary tooth in the anterior maxilla with the crown positioned towards the nasal floor. The maxillary CT demonstrated a quite close relationship of the tooth with the nasal cavity, so a nasal approach was planned.

Results: Under general anesthesia the supernumerary tooth was removed by means of an endoscopic approach from a nostril. During the extraction the adjacent structures were unharmed. The postoperative course was uneventful.

Conclusions: This case report suggests that in case of supernumerary teeth positioned close to the nasal cavity, trans-nasal endoscopy may represent a valid alternative to more demolishing traditional surgery.

\section{5}

\section{Preoperative Vascular Evaluation of Lower Extremity for Free Fibula Flap Transfer}

\section{Morganti, A. Bozzetti, P. Salvatori", D. Sozzi}

Department of Maxillo-Facial Surgery, Ospedale San Gerardo, Monza, Ospedale Niguarda, Milano, University of Milan-Bicocca (Head Professor A. Bozzetti), "Department

of Cervico-Facial Surgery, Ospedale San Giuseppe, Milano

Introduction: In international literature there are controversies about preoperative vascular assessment in fibular free flap. This study proposes a critical analysis of different preoperative evaluation and imaging (clinical examination, doppler, doppler ultrasonography, RM-angiography, CT-angiography, arteriography) and an accurate analysis about the advantage doppler ultrasonography (functional evaluation) and CT-angiography (fibular and arterial anatomical evaluation) association for preoperative imaging.

Materials and Methods: This study is a retrospective review of 80 patients evaluated for fibular free flap transfer between 2000 and 2008(age range 23-82 years,average age 58 years), 55 studied by clinical examination and doppler and 25 by clinical examination, doppler ultrasonography and CT-angiography.

73 patients underwent fibular reconstruction after oncological resection for malignant pathology, 4 for benign pathology, 2 for preprosthetic-surgery and 1 for a post-traumatic mandibular defect (shotgun trauma).

Results: Preoperative evaluation by clinical examination, doppler ultrasonography and CT-angiography influenced 5 surgical planning on 25 patients. We found 2 cases of deep venous insufficiency, 2 of arterial calcifications and 1 case of magna peroneal artery. In the sample evaluated only by doppler we found 1 preoperative exclusion and 1 case of intraoperative aberrant arterial anatomy (magna peroneal artery) in which surgical planning must be changed during operation. All patients underwent smooth free fibula transfer without ischemic complication in both recipient and donor site.

Conclusions: Preoperative evaluation with a low morbidity clinical examination with doppler ultrasonography and CT-angiography allows a more accurate knowledge of vascular anatomy compared to doppler use only. These examinations help the surgeon to prevent potential complications in patients with vascular anomalies and normal pedal pulse, decrease dissection time and to improve morphological and functional results.

\section{6 \\ Osseointegrated Implants Placed in Fibula- free Flaps: A 1- to 7-Year Retrospective Study \\ G. Pellegrino, M. T. Macchia, G. Lizio, G. Corinaldesi, A. Bianchi, C. Marchetti \\ Oral and Maxillofacial Surgery Unit, Department of Odontostomatological Sciences, University of Bologna, Italy}

Aim: To evaluate the survival and success rates of implants placed in reconstructed jaws with fibula-free flaps, and the differences between the clinical outcome of the native fibula-free flap and vertically distracted fibula-free flaps and the differences between the different kind of perimplants mucous.

Materials and Methods: 9 patients, reconstructed with fibula free flap were riabilitated with an implant-supported prostheses. Distraction osteogenesis (DO) was performed on 5 patients. At a mean of 31,5 months after jaw reconstruction, 44 implants were inserted and fixed-prostheses or overdenture were relieved. A skin or oral mucosa grafting was accomplished in 7 patients. The follow-up period averaged 48.5 months. Data on the course of DO and the clinical and radiographic outcomes of the implants were assessed.

Results: 4 implants failed during the follow-up period, all placed in distracted fibula free flaps without grafts. The mean periimplant bone resorption was $1.63 \mathrm{~mm}$ The cumulative implants survival and success rates were 90.9 and $65.85 \%$ respectively. In distracted and not distracted fibula the success rate was $60,71 \%$ and $76,92 \%$ respectively. The implant success rate after a skin graft placement was $88,8 \%$, after a palatal graft placement $84,21 \%$.

Discussion: Implants placed in fibula free flaps obtained an high survival rate. Distraction osteogenesis of fibula-free flaps had a significant number of complications and marked bone resorption around the implants. There were a high number of complica- 
tions of perimplant mucosa: corrective soft tissues surgeries are required, associated with accurated oral hygiene.

\section{7 \\ Quality of Life and Functional Status in Head and Neck Cancer Patients: Does Quality of Life Predict Long-Term Survival?}

\section{A. Tarsitano, A. Pizzigallo, C. Marchetti \\ Chirurgia Maxillofacciale, Policlinico S.Orsola-Malpighi, Università di Bologna, Italia}

Introduction: During the past 20 years, there has been increased awareness of quality of life as an outcome measure of head and neck cancer management. More recently, there has been interest in HR-QoL as a predictor of survival. We conducted this prospective longitudinal study to evaluate QoL and function of patients with head and neck cancer in relation to age, tumour location, stage and treatment modality.

Materials and Methods: We assessed quality of life association with long-term survival in this group of patients. 128 patients with head and neck cancer were evaluated with standardized HRQL questionnaires, the EORTC QLQC30 and the H\&N35. Overall survival and odds of death were measured.

Results: Highest mean scores, indicating worse impact on well-being, were noted in the domains of speech, social eating, and senses. Dry mouth and sticky saliva both demonstrated high impact on quality of life for patients underwent postoperative radiotherapy. Quality of life impairment was worse in all of the domains for combination therapy versus surgery alone $(\mathrm{p}<0.005)$. Tumour location and TNM staging were both significantly associated with each of the quality of life domain scores. Changes in quality of life scores in this cohort of patients were correlated with overall survival.

Conclusions: This study has demonstrated an independent association between before and after treatment HR-QoL and overall survival.

\section{8 \\ Management and Approaches in Anterior Frontal Sinus Fractures}

\section{E. Rebuffini, D. Rocchetta, D. Valente, G. Spinelli \\ Department of MaxilloFacial Traumatology, Traumatologic and Ortopaedics Center, Careggi Hospital, Florence, Italy}

Frontal sinus fractures are rare (5\%-15\%) and their management is still controversial: it ranges from observation to frontal sinus cranialization. The authors present their experience in treatment of isolated and depressed anterior wall fractures of the frontal sinus, without associated injuries of nasofrontal duct and posterior wall.
From January 2009 to April 2010, ten patients (age range: 18-68yrs) with a diagnosis of isolated anterior wall fracture of the frontal sinus were admitted to our department. CT scans with 3-D reconstruction were requested. Six patients, with comminuted anterior frontal sinus fracture, underwent surgical treatment with coronal approach or pre-existing frontal wound; bone fragments were reduced and fixed with microplates. Four patients, with isolated and displaced anterior frontal sinus fracture, at maximum two pieces, underwent a close percutaneous reduction with a long screw put through a small skin incision. The bones were kept in place only with reciprocal contact.

Post-operatory period was regular in all the patients and no major complications were observed. Patients underwent postoperatory CT-scan and clinical examination. In 8 cases the shape of anterior frontal sinus bone was completely restored with a good aesthetic result; in 2 patients a little asymmetry was observed.

Treatment of isolated anterior frontal sinus fracture depends on kind of fracture: comminuted fracture can be treated with "open" surgical access while isolated and displaced fracture with percutaneous close reduction. In all cases the surgical result is to obtain a fine aesthetic result.

\section{9}

\section{Lipofilling's Role in Facial Wasting Rehabilitation of HIV + Patients}

\author{
R. Rauso', V. Sangiovanni², G. Cobellis ${ }^{3}$, G. Tartaro ${ }^{1}$ \\ ${ }^{1}$ Head \& Neck Dept., II University of Naples, Naples, Italy, \\ 2"Cotugno", Infective Disease Hospital, Neaples, Italy, \\ ${ }^{3}$ Biology Dept., II University of Naples, Naples, Italy
}

Aim: The development of effective antiretroviral therapies for the treatment of human immunodeficiency virus (HIV) has drastically changed management for infected patients, with treatment approaches now similar to those of chronic disease rather than fatal illness. However, these therapies are not without side effects, some of which can be quite debilitating. One medication-associated condition that has become prevalent among HIV-infected patients is HIV-associated lipodistrophy, a syndrome characterized by abnormal fat metabolism and deposition.

Materials: Six-teen HIV+ patients affected by lipodistrophy, having antiretroviral terapie, were enrolled in this clinical retrospective study. Facial rehabilitation of patients was performed with lipofilling, harvesting fat from lipohypertrophyed areas. For each patient, an ultrasonographic examination of facial filled areas was conducted one and six months after surgery to control fat resorption.

Results: No infection or others complications occurred. The mean follow-up time for each patients was 14 months, photographic evaluations showed stable aesthetic results with no resorption of the transplanted fat as demonstered by ultrasonographic examinations.

Discussion and Conclusion: The use of the peripheral hypertrophied fat, harvested with cannulas, to restore the hypotrophized areas, such as the face, theoretically seems the best option achievable, however different results have been reported in literature. In our experience, fat harvested with cannulas with 
Coleman's technique and then infiltrated in the face is an effective technique to restore facial features in patients affected by HIV facial wasting. Ultrasonographic examinations performed one and six months after surgery showed no resorption of transplanted fat and an increased ultrasonographic intensity of it.

\section{0 \\ Management and Surgical Approaches in Isolated Blow-Out Fractures}

D. Valente, D. Rocchetta, E. Rebuffini, G. Spinelli

Introduction: Blow-out fractures of the orbital floor are typically caused by direct blunt trauma to the orbit. The predictability of the surgical treatment is closely related to the surgical access, timing and materials. The authors present their experience in treatment of blow-out fractures.

Materials and Methods: From January 2009 to March 2010, 39 blow-out fractures of orbital floor (30 male, 9 female, age range $18-80 \mathrm{yrs}$ ) were treated at our department. CT was requested for all patients, but only the patients with diplopia or entrapment of the inferior orbital muscle underwent ophtalmologic evaluation with Hess-Lancaster's test. Surgical indications were diplopia within 7 days, entrapment of inferior orbital muscle, enophtalmos over $2 \mathrm{~mm}$, defect area greater than $2.5-3 \mathrm{~cm}^{2}$. Surgical approach depended on age's patient, fracture's width, eyelid laxity, presence or absence of skin wound.

Results: Clinical follow-up was at 1, 3, 6 months. CT was required at 6 months after surgery. We used 3 different surgical access: subciliary (66\%), transconjunctival approach $(32 \%)$ and through a skin wound (2\%). Reconstruction of orbital floor was made with alloplastic material (Medpor ${ }^{\circledR}$ ) in $94 \%$ of cases, in other cases we used calvarian bone graft, autologous or omologous. There were not complications in immediately postoperatory period. Mean follow up was 6 months. Better aesthetical results were obtained after six months using a trans-conjunctival surgical approach.

Conclusion: We prefer the transconjunctival approach is better in the orbital floor's defects under $3,5 \mathrm{~cm}^{2}$, in cases of eyelids laxity or eyelid swelling; in the other cases a subciliary approach allows better surgical view.
351

\section{The Submental Endotracheal Intubation for the Tumours of the Upper Jaw}

\author{
M. Merlini ${ }^{1}, 2$, V. Palazzolo ${ }^{1}$, S. Valsecchi ${ }^{1}$, C. Gervasoni ${ }^{1}$, \\ S. Spotti', A. Di Francesco' ${ }^{1}$, L. Colombo ${ }^{1}$ \\ 'Department of Maxillofacial Surgery Sant'Anna Hospital, \\ Como, Italy, ${ }^{2}$ Department of Maxillofacial Surgery S. \\ Gerardo Hospital, Faculty of Medicine, Bicocca University \\ of Milan, Monza, Italy
}

Introduction: Tumours of upper jaw often requires free or local flaps reconstruction. When we choose the temporal muscle flap, nasotracheal or oro-tracheal intubation could limits strongly surgeon's work and surgical plan.

The conventional solution could be to carrying out a tracheotomy, adding a significant risk of iatrogenic complications. Authors analysed the results of the Altemir tecnique in order to time for intubation, intraoperative and post-operative complications.

Materials and Methods: From October 2007 to October 2009 , the submental route was performed in 5 patients suffering of different neoplasms of the upper jaw. In all patients was planned a total or subtotal maxillectomy with reconstruction by an omolateral temporal muscle flap.

After standard orotracheal intubation with a reinforced tracheal tube, a $2 \mathrm{~cm}$ skin incision was made in the submental, paramedian region, directly adiacent to the lower border of the mandible. At the end of surgery, the tube was pulled back in the reverse order and the cutaneous and mucosal wound were sutured.

Results: The procedure was successfully completed in all the patients without any complications. The submental route improve the transoral surgical access, giving the opportunity to perform the temporal muscle flap harvesting without any impairment of the surgical field.

Conclusion: The submental route intubation is a fast, safe and reliable procedure and could be recommended in treatment of the tumours of the upper jaw when no long term control of the airway is needed. The cosmetic result of the extraoral incision required is good to excellent.

\section{2 \\ Simulation Guided Navigation: Proposal of a New Method to Improve Reproducibility in Orthognathic Surgery}

\author{
M. Simona, B. Giovanni, B. Alberto, M. Claudio
}

Due to the recent development of 3-dimensional technology, computer software is increasingly being used for diagnosis, analysis, data documentation, and surgical planning for orthognathic surgery.

We present a new method to transfer individualized 3D virtual planning of the patient using a navigation system in the operating room to improve reproducibility of the simulation. 
Materials and Methods: Our study group was composed by 10 consecutive patients affected by dentofacial deformities. All the patients have been investigated with a preoperative Cone-Beam CT of the facial skeleton and soft tissues. The DICOM files of the CT scans were loaded in Surgicase CMF 4.0 (Materialise ${ }^{\varpi}$ ) to get a $3 \mathrm{D}$ virtual reconstruction of the face (bony parts and soft tissues). The surgical procedure was performed virtually on the bony $3 \mathrm{D}$ model and the software elaborated consequently the postoperative appearance of patient's soft tissues. Than the virtual planning was loaded on the Stryker ${ }^{\circledast}$ iNtellec ${ }^{\oplus} \mathrm{t}$ Cranial Navigation System as a STL file. In this way we detected intraoperatively the position of the mobilized bony segments with the navigation system, checking their correct positioning. After surgery all the patients underwent a new Cone-Beam CT scan and this was matched with the preoperative $3 \mathrm{D}$ virtual planning, providing this way an evaluation of the repeatability of the procedures.

Results: The parameters we considered were patients average matching error and its standard deviation, the $<2 \mathrm{~mm}$ matching error percentage, namely the Reproducibility.

- The minimum matching average error was $0,28+/-0,77 \mathrm{~mm}$ while the maximum matching error is $1,99+/-1,01 \mathrm{~mm}$

- The averarage percentage is $86,5 \%$ ranged in an interval values from $77,5 \%$ to $96,2 \%$

Conclusion: The use of a surgical navigation system seems to improve the intraoperative repeatability of the simulated virtual program in orthognathic surgery. We found $86.5 \%$ mean preoperative surgical plan reproducibility with the assistance of simulation-guided navigation, compared to $80 \%$ mean reproducibility obtained in our previous group, in which no intraoperative navigation was performed. According to these results, we can assume that simulation-guided navigation would be a helpful procedure during orthognathic surgery to improve reproducibility of the preoperative virtual surgical planning.

\section{3 \\ Simulation-Guided Navigation for Polyethylene Malar Implants Positioning in Surgery of Facial Deformities}

G. Badiali, S. Mazzoni, A. Bianchi, C. Marchetti

Aim: In this day and age, the surgical positioning of facial implants is mainly a matter of surgeon's capacity and artistic ability. From this point of view, polyethylene implants are very adaptable and are an effective tool for correcting facial deformities, mild to severe, without osteotomies or bone grafts. Anyway, there is a large room to improve these procedures in terms of surgical planning and aesthetic results. The aim of the study was to evaluate if surgical navigation technology could have a role in this process.

Materials and Methods: We present our experience in simulation-guided navigation for polyethylene malar implants positioning in four patients between December 2008 and February 2010. The clinical indications were congenital malar hypoplasia in syndromic patients (simultaneously or consecutively to orthognathic surgery) and acquired malar hypoplasia (monolateral or bilateral) caused by trauma or radiotherapy for neoplasm in pediatric age.

First, we performed the virtual simulation of the surgical procedure for each patient with Surgicase CMF (Materialise ${ }^{\varpi}$ - Leuven, Belgium). This software let us obtain soft tissue simulations of the postoperative appearance of the patient who undergoes facial bone surgery, using preoperative CBCT scans. This software also allows to simulate the positioning of an implant volume on the facial bones, equally showing facial soft tissue postoperative appearance.

Then, in order to intraoperatively perform the implant grafting in the exact position we planned preoperatively, we experimented a new procedure, based on the the use of a surgical navigation system (iNtellect Cranial Navigation System by Stryker-Leibinger ${ }^{\circledast}$ ). The virtual planning was loaded on the navigation system, which was used in the operating theatre to inset the implants on the facial skeleton, as a virtual surgical guide. All patients underwent postoperative CBCT scans in order to evaluate reliability of the surgical treatment.

Results: The aesthetic outcome of the four procedures was satisfactory. Postoperative evaluation, performed matching the simulated results with the postoperative CT scans, showed adequate reliability of the procedure. No implants was lost for complications.

\section{4 \\ Ridge Augmentation of the Atrophic Posterior Mandible with Inlay and Onlay Iliac Bone Grafting : A Prospective Controlled Clinical Trial for the Comparison of Two Techniques}

\author{
G. Lizio, P. Felice, G. Pellegrino, R. Pistilli, C. Marchetti
}


Conclusions: Inlay technique obtains lower bone resorption and more predictable outcomes but requires an experienced surgeon; Onlay technique obtains higher bone resorption, needs a bone block graft over-sized in height, but requires a shorter learning curve. Once implant placement has been carried out, the outcomes, comparable to those in native alveolar bone, are similar for both procedures.

\section{5}

\section{Open Reduction and Internal Fixation of a Subcondylar Fracture with a Trapezoidal Plate}

\author{
S. De Biasi, P. Piombino, A. Spinzia, A. Manfuso, \\ L. Califano
}

Department of Maxillofacial Surgery, "Federico II"

University of Naples, Italy, Chief: Prof: Luigi Califano

Introduction: The treatment of condylar fractures has always represented a challenge for the maxillofacial surgeon. Nevertheless several surgical approaches and osteosynthesis techniques have been widely described, a common consensus has not been obtained yet.

The aim of this study is to evaluate the stability of the trapezoidal plate for the fixation of subcondylar fractures.

Materials and Methods: From 2007 to 2009 we treated 4 cases of subcondylar fractures. The clinical evaluation was based on facial and oral examination and on orthopantomography and a maxillofacial Computer Tomography (CT). The surgery was performed under general anesthesia. The subcondylar fracture was exposed with the retromandibular approach that allows exposure of the entire ramus of the mandible including the condylar neck area. The Modus TCP plate (Modus TCPs 2.0, Medartis, Basel, Switzerland) used in these cases is a 4-hole titanium miniplate. After the surgery the patients received outpatient care services, for a period of 6 months.

Results: The cosmetic outcome was excellent. The occlusion was restored as before the injury. The ATM examination showed a good recovery of all mandibular movements. Orthopantomography and CT scan 6 months after the surgery showed the recovery of the mandibular ramus height and a good stability of the plate in all patients.

Conclusion: Retromandibular approach, associated with the features of the trapezoidal plate, should be considered as an effective alternative for the fixation of condylar neck and subcondylar fractures.

\section{6}

\section{New Technical Findings in Treatment of Orbital Floor Fractures}

\author{
G. Fianchini, P. Piombino, V. Abbate, A. Spinzia, L. Califano \\ University of Naples "Federico II", Via Pansini 5 I-80131, \\ Naples - Italy
}

Introduction: The aim of this study is to show our experience about the reconstruction of orbital floor fractures and new technical findings.

Materials \& Methods: At the Department of Maxillofacial Surgery of the Federico II University of Naples, Italy, we evaluated 30 patients with blow-out orbital fractures treated, from 2005 to 2007.

According literature, the indications for surgical treatment are persisting diplopia, enophthalmo, altered ocular motility, and infraorbital nerve hypoanesthesy.

An innovative preoperative examination by CT scans gave us the opportunity to classify the orbital floor fractures into small and large by measurement of the bone defect in order to choose the appropriate implant materials for orbital floor reconstruction .

All patients were treated with subciliary incision using reasorbable or non-reasorbable materials respectively in small and large fractures.

Results: It was observed a resolution of preoperative symptoms associated to a non evident skin scar outcome and the absence of postoperative complications.

Conclusion: We concluded that the use of resorbable materials for small orbital floor fractures and non-resorbable materials for large orbital floor fractures, according with our classification, provides reliable and reproducible stabilization of orbital wall defects, offering satisfactory results both functional and aesthetic. Furthermore this study suggest that our new technical findings allow to standardize the adoption of an appropriate implant materials in orbital floor reconstructions.

\section{7 \\ Recovery of Mandibular Functionality After Closed Treatment of Mandibular Condylar Fractures with a Modified Bionator of Balters}

\author{
S. Ferrara, P. Piombino, C. Sbordone, A. Spinzia, L. Califano \\ Department of Maxillofacial Surgery, "Federico II" \\ University of Naples, Italy, Chief: Prof: Luigi Califano
}

Introduction: Between $17.5 \%$ and $52 \%$ of all mandibular fractures are in the condylar region. The choice among either surgical or conservative treatment depends on the age of the patient, general clinical conditions, level of the fracture, displacement of the condylar fragment, dental occlusion and TMJ functionality. Despite the treatment applied, patients show a reduction of mandibular movements. Purpose of the present study is to evaluate the role of a modified Bionator of Balters, in the recovery of 
mandibular functionality after conservative treatment of condylar fractures.

Materials and Methods: Eight patients with intracapsular mandibular condylar fractures were observed in the Department of Maxillo-Facial Surgery University "Federico II" of Naples between 2007 and 2009. All patients underwent conservative treatment. The post-treatment mandibular movements were measured: interincisal opening, right and left lateral movements and protrusion. In addition the presence of lateral deviation on opening and protusion was evaluated. A modified Bionator of Balters was applied for 6 months and the patients underwent follow-up to evaluate the mandibular functionality. The modified Bionator of Balters is an orthodontic activator, it has to be designed and built, in the case of a bicondylar fracture, with the mandible in symmetrical protrusion, and in the case of a monocondylar fracture, with a mandibular lateral deviation opposite to the injury.

Results: After 6 months of therapy the patients showed the full restoration of mandibular movements.

Conclusions: The use of modified Bionator of Balters after conservative treatment of condylar fractures is important for the complete restoration of mandibular movements.

358

\section{Closure of Large Perforation of Sinus Membrane}

\section{Clementini, C. Pandolfi, C. Agrestini, P. Bollero, A. Barlattani \\ Dipartimento Scienze Odontostomatologiche, Università Tor Vergata, Roma}

Background: Maxillary sinus floor elevation is a well-documented technique and it is generally accepted as a regenerative procedure to facilitate dental implants placement in the posterior atrophic maxilla. Although the sinus lift procedure is relatively safe, some potential problems could be occur. The most prevalent intraoperative complication is perforation of sinus membrane, which can lead to graft infection and early failure. Various techniques and materials have been developed for repairing perforated sinus membrane.

Material and Methods: Various techniques aimed to close such perforations are presented and a case report is descripted, focused on a $7 \mathrm{~mm}$ perforation of sinus membrane occurred during preparation of the sinus window. The obliteration of the perforation was obtained through suturing sinus membrane with a resorbable material to the periosteum directly lateral to the osteotomy site, giving the chance to not abort sinus augmentation procedures althought a collagen membrane wasn't available.

Results: No serious infections have occured and clinical and radiographic findings at 4 months were adequate. These techniques result useful to obtain the closure of occurred perforation, providing the clinician with a containing element for the placement of the planned regenerative materials.

Conclusions and clinical implications: It can be concluded that membrane elevation must be carefully executed to avoid membrane perforation, but that if it occurs it is still pos- sible to continue the procedure safely using a variety of resorbable materials for the closure of the perforation.

\section{9}

\section{Oral Leukoplakia: Clinical and Molecular Features}

G. Nicolai, F. Luciani, F.N. Bartuli, F. Carrafiello, B. Lore, L. Calabrese

Università Degli Studi Di Roma "Tor Vergata", Cattedra Di Chirurgia Oro-maxillo-facciale, Scuola Di Specializzazione in Chirurgia Odontostomatologica, Direttore Prof.

Leonardo Calabrese

Introduction: Histologically, oral dysplasia is a potentially precancerous lesion. The progression risk is associated with the histological grade and it is unpredictable. Among the large range of these lesions, leukoplakia is the most common in terms of diagnosis and therapeutic management.

Material and Method: It is also important to establish a differential diagnosis with the other most important oral diseases and analyze the different clinical, and molecular features to give an idea of the malignant transformation risk. We studied the expression of podoplanina as a marker for the development of oral squamous carcinoma in 12 patients showing oral precancerous lesions.

Results: In four (33\%) of the 12 patients the basal and suprabasal layers were classified as podoplanina positive. Podoplanina positivity was more frequent in older patients $(\mathrm{P}=.019)$, females ones $(\mathrm{P}=.018)$, and dysplastic lesions $(\mathrm{P}=.048)$. Podoplanina positive OPL had a significantly higher risk to develop an oral cancer than the podoplanina negative ones.

Conclusion: Data in our possession are still premature, but the critical analysis of literature and the new discoveries in molecular biology give us the possibility to consider podoplanina a significant predictive marker of the development of precancerous lesions. 


\section{Obesity Surgery}

\section{0}

Effects of Biliopancreatic Diversion on Type 2 Diabetes in Patients with BMI Range of 25.0-34.9 $\mathrm{Kg} / \mathrm{m}^{2}$

\section{C.C. Campisi, G. D'Alessandro, A. Weiss, F. Pagliardi}

Operative Unit of General, Functional and Metabolic Surgery, Department of Surgery (DISC), University

Hospital «San Martino», Genoa, Italy

Objective: Biliopancreatic diversion (BPD) resolves type 2 diabetes in near totality of morbidly obese patients (BMI $\geq 35 \mathrm{~kg}$ / $\mathrm{m}^{2}$ ); an effect, accompanied by normalization of serum triglyceride and cholesterol. However, studies of BPD effect in BMI range 25.0-34.9 $\mathrm{kg} / \mathrm{m}^{2}$, including about $90 \%$ of diabetic patients, are lacking.

Materials and Methods: 30 type 2 diabetic patients with BMI 25-34.9 were submitted to BPD and monitored 12 months. 38 diabetic patients selected from a large database, kept 1 year on medical therapy, served as controls.

Results: M/F 19/11. Mean age 56.4 \pm 7.4 years, weight $84.8 \pm 11.1 \mathrm{~kg}$, BMI $30.6 \pm 2.9 \mathrm{~kg} / \mathrm{m}^{2}$, waist circumference $104 \pm 9.4$ $\mathrm{cm}$, diabetes duration $11.2 \pm 6.9$ years, $\mathrm{HbA}_{1 \mathrm{c}} 9.3 \pm 1.5$. 12 patients on insulin. 15 (2 F) with BMI $<30$ (mean: 28.1). BMI progressively decreased, stabilizing around 25 since the fourth month, without any excessive weight loss. 1 year after $\mathrm{BPD}$ mean $\mathrm{HbA}_{1 \mathrm{c}}$ was $6.3 \% \pm 0.8$, with 25 patients $(83 \%)$ controlled (HbA1c $\leq 7 \%$ ) on free diet, without antidiabetics, and the remaining improved.

Conclusions: BPD improves or resolves diabetes also in BMI 25-35 without causing any excessive weight loss, its action being on both insulin sensitivity and beta-cell function.

\section{1 \\ Revisional Bariatric Surgery After Failed Vertical Banded Gastroplasty}

\section{A. Franceschi, M. Ciciliot, R. Galleano, B. Rossi, F. Falchero \\ Santa Maria di Misericordia Hospital, Albenga (SV)}

Introduction: Long term complications after vertical banded gastroplasty (VBG) for treatment of morbid obesity have been reported and patients sometimes need revisional bariatric surgery (RBS). Most common cause is weight regain due to gastrogastric fistula. Laparoscopic Mac Lean procedure has lessened gastric complications even if inadequate weight loss or intolerance to solid food may happen. Conversion of VBG in gastric by pass (GBP) may be a therapeutic option.

A retrospective study was conducted to validate the efficacy and safety of RBS with conversion of VBG in GBP.
Materials and Methods: Between January 1996 and December 2007, 192 patients underwent VBG. Twelve subsequently needed RBS. Most common indication to reoperation was gastrogastric fistula. All patients were females (mean age 47 years). Mean weight and BMI were $106 \mathrm{Kg}$ and $43,2 \mathrm{Kg} / \mathrm{m} 2$.

Patients data from reintervention and follow up were reviewed to determine morbidity rate, modification of weight, BMI and comorbidities, and occurrence of long-term complications.

Results: One patient with a previous Mac Lean procedure was treated by laparoscopy; the others underwent a laparotomic operation. Mean operative time was 206 minutes. Mean hospital stay was 10 days. Major morbidity rate was $8 \%$ (1 gastric fistula treated conservatively) and minor one was $25 \%$ (2 respiratory complications, 1 wound infection). Mean follow up was 21 months (BMI 28,4 Kg/m2). Three incisional hernias developed at follow up.

Conclusion: RBS with conversion in GBP after failed VBG is a safe and efficient procedure in reducing weight and solving comorbidities with an accettable morbidity rate.

\section{2 \\ Endoscopic Finding of Gastric Remnant in Long Term Follow-up After Roux-en-y Gastric Bypass}

\section{Leuratti, M.P.L. Di simone*, S. Cariani}

Department of Emergency-Urgency, General Surgery and Transplantation, Obesity Surgery Center, University of Bologna, " Department of Emergency-Urgency, General Surgery and Transplantation, surgical endoscopy unit

Background: Roux-en-Y Gastric Bypass fashioned on a Vertical Banded Gastroplasty (RYGB-on-VBG) is a modified bariatric technique where a small communication between gastric pouch and gastric remnant allow upper routinely endoscopic study without negative effects on weight loss. Thanks to this solution is possible to detect early mucosal changes during follow-up.

Methods: From January 2009,45 asymptomatic patients who underwent RYGB-on-VBG in 2002-2006, were enrolled for standard upper endoscopy. All procedures were performed in oral sedation with Olimpus Video Gastroscope GIF-Q 165(Ø 9,2 mm). Biopsies of corpus, angulus and antrum were collected with standard biopsy forceps.

Results: Traditional endoscopy of remnant showed 4normal aspect (8.8\%), 22mild gastritis (48.8\%) and 19 severe (42.2\%) gastritis. Hystological analysis confirmed 1(2.2\%)normal pattern,while pointed out 3 intestinal metaplasia (6.6\%), 2 lymphoma-like gastritis (4.4\%),23 cronic gastritis (51.1\%) and 16 active gastritis $(35.5 \%)$

Conclusions: Excluded stomach after RYGB seems to develop pathological mucosal changes for multiple risk factor such as biliary reflux and physiological atrophy due to complete jejunal diversion of cud. Further studies are necessary to state natural evolution of these changes but it suggests the importance of bariatric surgical technique which allows the endoscopic study of gastric remnant. 
363

\section{Intragastric Balloon (bib ${ }^{\circledR}$ ) Associated with Liposuction: a Preliminary Evaluation on Safety and Synergistic Effecto on Mild Obesity}

\section{S. Tramontano, C. Borriello, V. Pilone, A. Monda, \\ S. Gargiulo, E. Villamaina, P. Forestieri \\ Dipartimento di Chirurgia Generale, Geriatrica, Oncologica e Tecnologie Avanzate- Università degli Studi di Napoli "Federico II"}

Before and in preparation for bariatric surgery, treatment with endoscopic intragastric ballon $\left(\mathrm{BIB}^{\circ}\right)$ represents a low risk procedure, with behavioral effect. Plastic surgery is a crucial step in the management of bariatric patients; a synergistic effect was evaluated in a preliminary study.

Group A consisted of patients with BMI between 30 and 40 , without disease for which the BIB $^{\circ}$ is contraindicated, and with significant representation of the subcutaneous fat in buttock region and thighs, in which was performed $\mathrm{BIB}^{\circ}$ placement (with standard technique) and the megaliposuction, to draw 5000-8000 $\mathrm{ml}$, after infiltrating a standard solution. Two control groups were evaluated: group B, including only patients undergoing BIB', and group C, performing only liposuction. Safety, complications and results were evaluated and compared among groups.

In group A twelve patients was enrolled. Case and control groups resulted homogeneous. No major complication was observed. Group A presented a better profile of weight loss compared with controls on all follow-up steps, significant after 2, 3 and 4 months. There was not significant difference for post-procedure discomfort. The HOMA index and mean cholesterolemia were significantly reduced in group A at 2 and 3 months.

While bariatric endoscopic treatment is a consolidated option, integration with the treatment of localized fat is an option of great prospect, if safety and improvement of the results will be confirmed on a large scale. Our assessment is the first experience in this direction. The synergy was observed in the overall improvement in performance after BIB" at all follow-up.

\section{4}

\section{Gastric Erosion After Laparoscopic Gastric Banding: Analysis of External Factors on a Single Surgical Group Among Different Operating Rooms}

\section{S. Tramontano, V. Pilone, A. Monda, A. Formato, L. lacobelli, E. Villamaina, M. Perone and P. Forestieri \\ Dipartimento di Chirurgia Generale, Geriatrica, Oncologica e Tecnologie Avanzate- Università degli Studi di Napoli "Federico II"}

Stomach erosion (SE) after gastric banding represents one of its least studied complications, despite modalities of presentation and treatment have been clarified in recent years. Associations between this complication and specific surgical factors associated with banding have not been reported. Our study evaluated the incidence of this complication in various operating environments, in order to identify a possible association with a specific parameter in the perioperative preparation.

A total of 250 patients underwent gastric banding between 2006 and 2008 and were followed for at least one year of follow-up. The procedure was performed in three Centers by the same surgical team. All banding were performed with standardized techniques. The data from the three Centers were evaluated comparatively, in order to assess the incidence of SE and compare the instrument sterilization and perioperative preparation techniques.

Between May 2006 and December 2008, 140 patients operated on at Center A, 70 at Center B and 50 at Center C were included. 12 cases of SE were observed (4.8\%): two at Center A (1.4\%) and 10 at Center C (25\%; A vs. C and A vs. B;p<0.05). No significant difference in sterilization techniques was observed, while there were differences in perioperative preparation and preparation of the operating table instruments (although not objectifiable parameters).

Among the etiological factors of SE, the environmental factor appears to be significant in the majority of cases. This finding highlights the need for a search for a new physiopathological examination in analyzing this complication.

\section{5}

Gastric Emptying After Sleeve Gastrectomy. Scintigraphic Assessment in a Preliminary Study

A. Monda, V. Pilone, S. Tramontano, E. Villamaina, M.G. Caprio, A. Formato, and P. Forestieri

Dipartimento di Chirurgia Generale, Geriatrica, Oncologica e Tecnologie Avanzate- Università degli Studi di Napoli

"Federico II"

Introduction: Vertical gastrectomy (sleeve gastrectomy SG) is spreading as an isolate bariatric procedure, with a failure rate at 5 years of about $30 \%$. Yet discussed is the effect on gastric transit, related also with a neurohormonal rebalancing.

Materials and Methods: Our preliminary study evaluated the effect on gastric emptying, comparing the transit times before and after SG and with a control population. Before SG and after 3 -4 months the patients had a semisolid standard meal. $T 1 / 2$ of the tracer was calculated in all patients, while weight loss at 1,3 and 6 months after surgery.

Results: A total of 15 patients were enrolled between cases and 20 patients in the control group. Between the two groups there were no anthropometric differences, except for BMI. All SG were completed laparoscopically, with standard technique. No major complications was reported. The mean\% EWL was 7.5. 19.8 and 35.7 , respectively at 1,3 and 6 months. During preoperative time, there were no abnormal value in transit time. At a mean f.u. of 10.4 months weight regain was not observed. Scintigraphic study showed a not significantly reduced $t \frac{1}{2}$ in case group $(80.4 \pm 16.5 \mathrm{vs}$ $64.3 \pm 22 \min \mathrm{p}=0.06)$. 
Conclusion: Our data demonstrated a reduction of the emptying of the stomach, but not statistically significant. Further enrollment and acquisition of long-term analysis will confirm whether the SG has, among its effects, an accelerated gastric emptying.

\section{6 \\ Laparoscopic Gastric Bypass: Control of Wide Gastro-Jejunal Anastomosis After Two Years Follow-Up}

M.B. Ciciriello, M. Giuffrè, V. Borrelli

Unit of laparoscopic and bariatric surgery. "S. Scolastica"

Hospital - Cassino - Italy

Background: The rapid arrival of food directly in the ileum, causes a reduction in appetite and the appearance of post-cibal syndrome, is one of physiopathological hypotheses regarding the mechanism of action of Gastric Bypass procedure. A wide gastro-jejunal anastomosis (GJ) with a small gastric pouch would guarantee gastric emptying and food transit in the intestinal side rapid enough to reach ileum and carry out its function of anorexic stimulus.

Materials and Methods: From September 2007 to September 2009 were performed 20 Laparoscopic Gastric Bypass (GBPL) with a antecolic antegastric GJ anastomosis with a 45 $\mathrm{mm}$ linear stapler on the anterior or posterior gastric-pouch wall. Endoscopic and Barium Rx control were made after 12 and 24 post-operatively months for evaluated the volume of gastric pouch and the size of GJ anastomosis.

Results: No stenosis of GJ anastomosis were found. In all cases endoscopic control showed a very small gastric pouch and a large GJ anastomosis. Barium Rx evidenced as well a very small gastric pouch as a rapid transit of barium into intestine.

Conclusion: Gastric pouch remain small after two years in pts underwent GBPL with a wide GJ anastomosis. Additionally food transit from gastric pouch into intestine is rapid thanks to the size of GJ anastomosis.

\section{7}

\section{Oversight in Performing the Jejuno-Jejunal Anastomosis During Laparoscopic Gastric Bypass}

\section{M.B. Ciciriello, V. Borrelli \\ Unit of laparoscopic and bariatric surgery. "S. Scolastica" \\ Hospital - Cassino - Italy}

This video shows a mistake in performing the jejuno-jejunal anastomosis, during laparoscopic gastric bypass, caused by an oversight while positioning the linear stapler into the intestinal lumen.
After the measurement of the established length of the alimentary and biliary limb, an anchoring stitch between them is positioned and a small hole is produced on both the two limbs.

Once the dissector penetrates through the intestinal lumen, the branches of the linear stapler (vascular load) are introduced into the two intestinal limbs, the anastomosis is so performed.

Once the stapler is opened it is visible the complete cutting of the biliary limb, the closure of the alimentary tract and the complete lack of the anastomotic lumen! After a few minutes of "dazing" the matter comes clear: a branch of the linear stapler hasn't been introduced into the alimentary limb.

Therefore the alimentary limb is opened, and the anastomosis is performed beneath the former attempt, whith a $60 \mathrm{~mm}$ vascular linear stapler load.

Once ensured of the patency of the anastomosis, the wide defect is closed whit two vascular loads of linear stapler. After being sure to have left a wide intestinal lumen, the procedure is fulfilled.

\section{Orthopedic and Trauma Surgery}

\author{
368 \\ Surgical treatment of Ewing's sarcoma of \\ the Extremities in the paediatric age

\begin{abstract}
N. Ali, C. Errani, M. Manfrini, A. Biazzo, A. Toscano, M. Mercuri

Clinica Ortopedica E Traumatologica IVa Prevalente Indirizzo Oncologico, Istituto Ortopedico Rizzoli, Bologna, Italy
\end{abstract}

Introduction: In children, the primary malignant bone tumours represent $16 \%$ of the cases in the Italian Register of Bone Cancer. Ewing's sarcoma and osteosarcoma are the most frequent entities.

Material and Methods: From February 1977 to December 2005, 123 patients under 15 year old were treated for Ewing's sarcoma of the extremities at the Rizzoli Orthopaedic Institute. The median age was 8,3 . The tumour was slightly more common in males. The most common site of primary Ewing's sarcoma was the knee.

108 patients underwent to limb-sparing surgery (88\%), for 12 patients amputation was warranted, and 3 patients undergo to rotatinplasty.

Median follow-up was 45 months (range: 24-324 months)

Results: 80 patients remained continuously free of disease (65\%).

The functional outcome was better in patients who underwent limb sparing surgery and biological reconstruction (allograft shell and vascularised fibular graft) respect the use of metallic endoprosthetics or amputation.

Secondary amputation was warranted in 5 patients, 4 for a deep infection and 1 for radio-induced sarcoma. 
Conclusion: The development of multimodality treatment for bone sarcomas represents a major advance in the treatment of these tumours, allowing preservation of the limb function and improving the survival.

In the last years, the development of limb-sparing surgery, not only improved the survival of these patients but even their quality of life, considering the devastating implications that the los of a limb can have in a paediatric population.

\section{9 \\ Blood Loss in Total Knee Arthroplasty: Mechanical Vs Pharmacological Approach}

P. Antinolfi ${ }^{1}$, B. Innocenti ${ }^{2}$, A. Caraffa ${ }^{1}$, G. Cerulli ${ }^{1}$

\section{Chirurgia Ortopedica}

Objective: Assess blood loss reduction by post-op knee flexion and compare it with local injection of tranexamic acid. Second endpoint was to evaluate if local injection of tranexamic acid can influence trasfusion reqirements in TKA.

Materials and Methods: Three groups were randomized in order to riceive: 1) conventional intra-op hemostasis 2) intrarticular injection of $500 \mathrm{mg}$ acid tranexamic at the end of surgery and 3) three hours post-op flexed knee at 90 degree followed by three hours at 50 degree of flexion. Only primary total Knee were included, all cemented and using the same Total Knee system. Tranexamic acid allergia, Assumption of Aspirin, warfarin or other pharmacological therapy as well as any co-morbidity influencing the coagulation systems, previous surgery to the knee, (with the exception of meniscectomy) and important renal failure were exclusion criteria for each group. We checked the drainage level on day " 0 ", " 1 " and " 2 ", we compared the variations in Hemoglobin and hematocrit value to the pre-op value and we analized the variation in blood trasfusions needed in each patient.

Results: In Our evaluation Tranexamic acid reduced of about $48 \%$ trasfusion requirements, $28 \%$ of blood loss compared to control group and $15 \%$ compared to flexion group while $17 \%$ was the reduction of blood loss of flexion group compared to control.

\section{0}

Soft Tissue Sarcoma in Young Children: The Experience of the Rizzoli Orthopaedic Institute

\author{
A. Biazzo, C. Errani, E. Staals, A. Toscano, N. Ali, \\ M. Manfrini, M. Mercuri \\ IV Clinica di Ortopedia e Traumatologia a prevalente \\ indirizzo oncologico dell'Istituto Ortopedico Rizzoli- \\ Università di Bologna
}

Introduction: Soft tissue sarcomas are an heterogeneous family of tumours, that can arise in every site. This work has the goal to give guide-lines for the treatment of these rare pathologies.

Materials and method: Between 1981 and 2005 were treated at the Rizzoli Institute 57 cases of soft tissue sarcomas in children $(<15$ years). The most representative histotype was Embryonal rhabdomyosarcoma, followed by Synovial sarcoma and Ewing's sarcoma. The most frequent site was the thigh, followed by the leg and foot.

21 had previous treatment elsewhere; of these, 14 underwent limb-salvage surgery: scar excision in 11 cases and excision of local recurrence in 3. 7 cases underwent an amputation.

Among the others 36 cases, only 6 had a demolitive surgery, the others received a limb-salvage surgery, in association with bone reconstruction in 3 and vascular reconstruction in 1 case. Chemotherapy was applied to 33 cases and radiotherapy to 10 .

Results: The most important prognostic factor was histotype of tumour. The survival at 5 years was $100 \%$ for Congenital fibrosarcoma, 83\% for Synovial sarcoma and 38\% for Embryonal rhabdomyosarcoma.

Conclusion: The treatment of these kind of tumours is multidisciplinary, and involves in surgery, chemotherapy and radiotherapy.

These techniques could also be applied for therapeutic purposes and target therapies in the nearby future.

371

\section{Damage Control Orthopaedics: A Safe Bridge Between the Acute Phase of Trauma and the Definitive Surgical Treatment}

\section{L.B. Vergano, M Monesi \\ U.O. Ortopedia e Traumatologia, Ospedale M. Bufalini, Cesena (FC)}

Early stabilization of fractures decreases the incidence of Multi Organ Failure Syndrome and increases survival rate. In multipleinjured patients (expecially with thoracic and/or head trauma) an aggressive surgical approach increases the risk of pulmonary ARDS and, consequentially, worsens the prognosis.

In orthopaedic traumatology, in the last years, the concept of DCO has been associated to ETC (Early Total Care), expecially as 
a bridge between the acute phase of trauma and the final surgical treatment.

DCO, with temporary fixation of fractures, allows adequate care of soft tissues, controls bleeding from bone fragments, while treating other life threatening injuries, without causing further damage to the weak metabolic and immunologic omeostasis of politraumatized patients.

The most used surgical tecnique in DCO is the external fixation, considered fast, easy and safe. It stabilizes effectively fractures and dislocation, it maintains limb length, it minimizes blood loss and allows the conversion to definitive osteosynthesis when the general (or local) condition are suitable, without endangering the final outcome.

The emodinamically unstable or borderline patients have the most benefits from DCO, while stable patients can be treated both with DCO, both with ETC, depending on different situations (type of hospital, surgical equipe, availability of operating room, etc.).

\section{2 \\ Retroperitoneal Hematoma During Fondaparinux Treatment: When the Medical Error Is Behind the Corner}

\section{Fortina, S. Carta, D. Scipio, A. Riva, M. Mangiavacchi, P. Ferrata \\ Orthopaedics and Traumatology Clinic, Department of Human Patology and Oncology, Azienda Ospedaliera \\ Universitaria Senese}

Introduction: Spontaneous hematomas has been described during antithromboembolic treatment, expecially in elderly. Agerelated changes in the pharmacodynamic and pharmacokinetic properties of this drugs increase the risk of bleeding. Moreover elderly patients, despite a normal serum creatinine value, could have a significantly reduced creatinine clearance $(\mathrm{CrCl})$, that leads to a further increased risk of bleeding. This chronic renal failure increases the bleeding with various mechanisms that cause a spontaneous rupture of arteries. With a moderate reduction of $\mathrm{CrCl}$ even with normal serum creatinine values, the fondaparinux can duplicate its half-life leading to an increased risk of bleeding.

Materials and Methods: A 78 year-old man developed a retroperitoneal hematoma during fondaparinux treatment after a total hip replacement.

Results: He had normal serum creatinine but a reduced $\mathrm{CrCl}$ that only the complication has showed.

Conclusion: Fondaparinux is a safe and effective alternative to LMWH in the prevention of venous thromboembolism following major orthopaedic surgery, but it should be used carefully in elderly. In these high-risk patients not only serum creatinine, but also $\mathrm{CrCl}$ should be monitored. The Cockroft-Gault formula provides a valid and fast result (Level A) to early identify patients with unapparent renal failure and its use should became the routine.
373

\section{Combined Positron Emission Tomography Using Gallium 68 with Computed Tomography and Use of Antibiotic Containing Hydroxyapatite Pallets in the Treatment of Bone Infections}

\author{
C. Errani*, E. Staals*, A. Toscano*, A. Biazzo*, N. Ali, \\ C. Nanni", S. Fanti", P. Ruggieri*, M. Mercuri* \\ *IV Clinica di Ortopedia e Traumatologia ad indirizzo \\ oncologico, Istituto Ortopedico Rizzoli, Bologna, Italy, \\ "Medicina Nucleare, Ospedale Sant'Orsola Malpighi, \\ Bologna, Italy
}

Introduction: Therapy of bone infections is still one of the major challenges in orthopaedic surgery. The combination of antibiotics and absorbable scaffold is advisable for a gradual release, obtaining high concentrations of local antibiotics. Moreover, reliable and reproducible image-based tests are needed to plan the surgery and to monitor the outcome.

Materials and Methods: We retrospectively reviewed the records of 11 patients with bone infections of the limbs treated at Musculoskeletal Oncology Department of our institution. In all cases a Positron Emission Tomography using Gallium 68 and Computed Tomography (PET-CT G68) was performed. All patients underwent curettage of the lesion followed by intravenous antibiotics for at least six weeks. The cavity was filled with absorbable Hydroxyapatite (Perossal'), loaded with antibiotics. In two cases, besides a curettage, an arthrodesis was performed.

Results: At final follow-up, ten patients had no clinical signs of infection after a single surgical procedure. In all these cases, the PET-CT G68 was negative. One patient, who underwent only one surgical procedure so far, remains painful and shows positive uptake on PET-CT G68. Functional results were good to excellent in nine patients, and poor in both patients who underwent joint fusion.

Conclusion: The review of our series documents that after curettage, the association of Hydroxyapatite and local antibiotics proved to be a successful treatment for this challenging disease. Furthermore, there is a threefold role for PET-CT G68 in bone infections. First, for diagnosis; second, for surgical planning; lastly, to monitor the outcome.

\section{4 \\ Proximal Humerus Reconstruction in Bone Tumor Surgery: Osteoarticular Allografts vs Allograft-Prosthesis Composites}

\author{
M. Ippolito, D.A. Campanacci, F. Frenos, D. Matera, \\ A. Lorenzoni, R. Capanna
}

In proximal humerus reconstructions, osteoarticular massive allograft (OA) allow a biologic reattachment of tendineous structures of the host providing stability and functional recov- 
ery. Allograft-prosthesis composites (APC) are more technically demanding, but functional advantages with new prosthetic design are expected with lower fracture and failure rate with time. We compared the outcome of patients treated with OA reconstruction (group A) and patients treated with an APC (group B) after humerus resection for bone tumors.

Thirthysix patients treated between 1996 and 2009 at the Orthopaedic Oncology and Reconstructive Center of AOU Careggi-Florence were included in the study (18 group A; 18 group B).

In group $A$, at an average follow-up of 66 months (2-116): 89\% were continuosly disease free, $56 \%$ had no complication, in $33 \%$ we observed a fracture of the graft and in $17 \%$ a pseudoarthrosis. In group $B$, at an average follow up of 44 months (3-164), 89\% were continuosly disease free, $72 \%$ had no complication, $11 \%$ stem loosening, $11 \%$ dislocation, in 1 case pseudoarthrosis and in 1 case detachment of allograft's great tuberosity. Functional outcome was evaluated using MSTS scoring system: a mean score of 24 (13-30) in group A and 23 (15-27) in group B was observed.

In our experience, proximal humerus APC showed functional results comparable to $\mathrm{OA}$ allograft with lower complication rate.

\section{5}

\section{Soft Tissue Sarcoma of Adults, Pre or Postoperative Radio/Chemo Therapy: The Surgeons Point of View}

\author{
M. Ippolito, G. Beltrami, A. Lorenzoni, D. Campanacci, \\ R. Capanna
}

Department of Orthopedic Oncology and Reconstructive Surgery, AOUC Careggi, Firenze

On the base of our experience: 850 patients treated from 1994 to 2009 in our Orthopaedic Oncology and Reconstructive Center of AOU Careggi-Florence, we classify the adult soft tissue sarcomas (STS) in three categories: resectable tumors, borderline resectable tumors and unresectable tumors. When a tumor is primary resectable or in case of radicalization of inadequate previous surgery, we usually perform an adequate surgery associated, in high grade STS, to brachitherapy and postoperative Radiationtherapy (RT) and Chemotherapy (CT). A free or a rotational flaps often help to obtain a functional soft tissue coverage and adequate surgical margins. In case of wide tumors, close to neurovascular bundle or when a major reconstruction is needed (for example microvascular flap with vascular and neural anastomosis, nerve graft, vascular by pass etc) we consider these tumors borderline resection. In such cases we perform neoadjuvant $\mathrm{CT}$ and or RT in order to obtain a tumoral srinkhage and to reduce the surgical squeezing during operation. This is followed sometimes by Brachyterapy and several times by CT. In case of huge mass involving nerves, vessels and bone or when metastatic disease is contemporary to primary lesion, we use to treat these patients affected by "unresectable tumors" trough an iperthermic perfusion by TNF and chemotherapy, followed by surgery and or radiationtherapy.

\section{6 \\ Distal Extremity Fractures of Radio and Ulna: A New - Non Bridging - External Fixation Technique for the Wrist}

\section{E. Theodorakis, F. Laurenti, D. Potestio, P. Braidotti}

Dipartimento di Scienze dell'Apparato Locomotore, Policlinico Umberto I, "Sapienza" Università di Roma, Italy

Introduction: Distal extremity fractures of radio and ulna represent approximately the $17 \%$ of the fractures in the Orthopaedic Emergency Departments in Italy, which are often associated with other fractures that may request an immediate treatment .

Materials and methods : We began to treat some of these fractures with a new -non bridging- external fixation system (CASLAU) which is achieved by the insertion of two K- wires and two pins at the distal radio. In our study we report clinical and radiographic results in 100 patients for fractures type $\mathrm{A}, \mathrm{B}$ and $\mathrm{C}$ (AO classification) during the period 2008-2010, with a follow up of 3 to 12 months. DASH and PRWE questionnaires were also used to establish our clinical results.

Results: Most of the patients have had a good clinical/functional result and declare satisfaction for the results obtained immediately after removing this external fixation system. 60 days after E.F. removal, all of the patients had a very good clinical/functional result. This result was constant with minimal variations during a 3 to 12 month period.

Conclusion: This new external fixation system results an optimal alternative treatment in various types of wrist fractures including cases that demand an instant surgical approach. This method can be considered particularly indicated in the elderly patients due to the mini invasivity and the early mobilization of the wrist.

\section{7}

\section{Our Experience with Surgical Treatment of Metastases in Long Bones}

L. Di Giorgio, G. Touloupakis, F. Vitullo, L. Imparato,

L. Sodano, M. Nardi, C. Villani

Dipartimento di Scienze dell'Apparato Locomotore, Policlinico Umberto I, "Sapienza" Università di Roma, Italy

Introduction: The presence of metastasis in a long bone is a distressing and painful event.

Materials and Methods: A retrospective study of the results of operative treatment of long bones' secondary metastases in 51 patients was carried out. The most common primary tumors were lung (19 patients) and breast (10 patients). Long bone metastases were predominantly found in the femur $(61,3 \%)$ and in the humerus $(32,2 \%)$. The patients were divided into four classes according to Capanna's protocol. Many different surgical treatment techniques of skeletal metastases in long bones were used including arthroplasty or a combination of internal fixation with 
chemical adjuvants (phenol and polimetilmetacrilate) that provided immediate stability.

Results: The mean survival rate was 8.1 months. We also noticed a significant improvement in physical activity and rehabilitation in the investigated group.

Conclusion: The present study showed that the surgical treatment of long bone metastases according to Capanna's classification system provides lessening of pain, better nursing assistance and an increase in quality of life.

\section{8 \\ Modular Endoprosthetic Replacement of Lower Limbs: Experience at Orthopaedic Oncology and Reconstructive Center of Florence}

\section{A. Lorenzoni, M. Ippolito, D. Campanacci, R. Capanna}

Specialità: Chirurgia Ortopedica

In case of massive bone loss in the lower limbs it is possible to reconstruct whole scheletral segments through the use of modular endoprosthetics. For about 9 years our Orthopaedic Oncology and Reconstructive Center of Careggi-Florence has used the Megasystem C (Link), a modular endoprosthetic system that allows the reconstruction of the proximal,distal or total femur,also the proximal tibia. We performed a retrospective study of patients treated in our department between June 2001 and December 2008. A total of 254 operations were performed more than $50 \%$ were riconstructions proximal femoral, over $90 \%$ resections for primary or secondary tumours, $10 \%$ case load were prosthetic revisions or following traumatic lesions. Were reported the presence of infection (9\%), dislocation (7\%), aseptic loosening (2\%), mechanical failure with stem or morse taper breakage (3.6\%). The oncological patients followed in long term (follow-up ranging from 2-9 years ) $35 \%$ of the patients resulted countinously disease free, $37 \%$ were alive either with disease due to the appearance of local recurrence (17 cases,7.5\%) or metastasis; $5.3 \%$ died of oncological causes; 1 patient died of other causes; the remainder of the patients $(22 \%)$ were lost to follow-up. According to the MSTS functionaly score $33 \%$ of patiens was excellent and $41 \%$ good.

\section{9}

\section{Multiple Osteochondromas: A New Classification System and Results of the Genotype-Phenotype Study}
A. Toscano, N. Fabbri, L. Campanacci, C. Errani, A. Biazzo, N. Ali, M. Mercuri
IV Clinica di Ortopedia e Traumatologia ad indirizzo Oncologico, Istituto Ortopedico Rizzoli, Bologna

Summary/Conclusion: Most severe clinical features are associated with EXT1 mutations. Prevalence of more severe dis- ease was significantly more common in males. No prognostic factors for malignant change were identified.

Introduction: Multiple Hereditary Exostoses is a rare dominant autosomic skeletal chondrodysplasia characterized by variable number of osteochondromas and wide spectrum of clinical expression. Rarely, disease course is complicated by trasformation to secondary chondrosarcoma (1-5\%). Research in the field of genetics has lead to identification of 2 responsible genes (EXT1 and EXT2), both coding for transmembrane glycoproteins involved in the synthesis of heparan-sulfate chains. Purpose of this paper is to present a new clinical classification system and its application in a prospective genotype-phenotype study.

Methods: Multidisciplinary clinic, involving genetists and orthopaedic surgeons, is weekly carried out. Patients genealogy is investigated and genetic screening by DHPLC (Denaturing High Performance Liquid Chromatography) carried out along with clinical assessment using a new classification system. The latter is based on deformity, functional limitation, limb-length discrepancy and number of joints affected. A prospective longitudinal study currently enrolling 240 patients was undertaken in March 2003 to correlate genotype and phenotype of the disease in families and sporadic cases.

Results: The new clinical classification provided a useful and reliable tool to stratify patients. Most severe clinical presentations were significantly associated with EXT1 mutations and male gender. Significant correlation was found between number of affected metaphyses and severity of disease. No risk factors for malignant change were identified.

Discussion and Conclusion: Prospective genotype-phenotype study identified risk factors for more severe disease. It appears that longer follow-up may also improve patient subgroups identification and perhaps allow insight for factors possibly affecting malignant change.

380

\section{Surgical Treatment of Scapular Fractures}

E. Cesari, P. Paladini, F. Campi, G. Porcellini

Introduction: Fractures of the scapula are often indicative of high-energy trauma. . Scapular fractures may be classified as being extra (body, processes, neck) or intra-articular. The extra-articular fractures heal uneventful under a conservative regimen. Most intra-articular fractures are a Bony Bankart variety. They should be treated by internal fixation, if the size of the fragment is bigger than $25 \%$ glenoid area.

Materials and Methods: We treated 4 cases of scapular body fracture by a modified Judet approach that allowed for excellent scapular and glenoid fracture visualization and reduction while preserving rotator cuff function. In 25 sport-practicing patients we used an arthroscopic approach to a bony Bankart lesion less than 3 months old and involving less than $25 \%$ of the glenoid. We performed a modified Bankart technique to fix the avulsed bone fragment to the healthy glenoid rim using suture anchors.

Results: The modified Judet approach allowed in all cases a stable fixation along the lateral scapular border provided excellent 
plate position and fracture stability. Shoulder function and stability were restored in 23 patients (92\%) by 2 years after arthroscopic surgery. There was no recurrence of instability and range of motion was minimally reduced.

Conclusion: The arthroscopic technique seems to offer an optimal method for treating acute bony bankart less than $25 \%$ of glenoid.

\section{1}

\section{Treatment Principles in the Management of Open Fractures}

\author{
A. Campagna, C. Biagini, S. Landi, P. Razzaboni, F. Lijoi \\ Department of Orthopaedics and Traumatology, Morgagni- \\ Pierantoni Hospital, Forlì, Italy
}

Open fractures are complex injuries that involve both the bone and the surrounding soft tissues. Management goals are prevention of infection, union of fracture, and restoration of function.

Despite the improvements in technology and surgical techniques, rates of infection and nonunion are still troublesome. The classification of open fractures is based on type of fracture, associated soft-tissue injury, and bacterial contamination present, the most widely used is Gustilo classification. Techniques of fracture stabilization depend on the anatomic location of the fracture, characteristics of the injury, soft tissues conditions, neurological and vascular complications, and the timing of the fracture.

The treatment should involve the use of early, systemic, widespectrum antibiotic therapy, repeated thorough irrigation and debridement, early fracture stabilization, early wound closure, and aggressive rehabilitation. The result of treatment is largely affected by the initial soft-tissue loss, wound contamination, fracture stability, and neurovascular status.

\section{2 \\ Prosthesis Infections, Our Treatment Protocol}

\section{Bove, D. Attala, P.Persiani, C. Villani \\ Clinica Ortopedica Eac09 La Sapienza Università di Roma}

Introduction: In past years there was seen an increment of hip and knee arthroplasty surgery in Italy, passing from 48531hip implants in 2002 to 55868 implants in 2005 and 30988 knee implants in 2002 to 44119 implants in 2005. The same increment was seen also in the U.S.A and in Sweden. Hip and Knee infection rate in Italy was 2276 in 2005 while in the U.S.A and in Sweden there were 120,000 and 337 respectively.

Material and Methods: In order to Approach prosthesis infection we designed in 2006 a treatment protocol and applied it on 24 patients from which 20 hips ( $80^{*}$, mean age 75 , and 12, mean age 70 ) and 4 knees hips ( $20^{\circ}$, mean age 70 , and $2 \%$, mean age 74). the patients underwent on a two stage treatment and had been evaluated with SF-36, WOMAC and HHS questionnaires.

Risults: 22 patients had a complete recovery with followup of 1-2 years (1 hip and 1 knee had a complete recovery after 1 year) with a satisfying Quality of life (QoL). We have noticed range of motion limitation in one knee $\$$ and leg length inequality in one Hip?.

Conclusion: Total hip and knee arthroplasty represents very successful and effective interventions in terms of QoL. On the other hand, post operative infections reduces patients QoL and increases the cost of national health service $(30,000 \$$ a patient in the USA). The goal of each surgeon is to diagnosticate as soon as possible the infected site and start the most suitable treatment in order to resolve the infection.

\section{3}

\section{Necrotizing Fasciitis of the Lower Limb: A Case Report}

\section{Biagini, A. Campagna, P. Razzaboni, S. Landi, F. Lijoi}

Department of Orthopaedics and Traumatology, MorgagniPierantoni Hospital, Forlì, Italy

Introduction: Necrotizing fasciitis (NF) is a severe, progressive soft-tissue infection, characterized by a rapidly spreading inflammation, with necrosis of the fascia and tissues. It is mostly caused by group A beta-haemolytic streptococci or multibacterial.

Materials and Methods: A 66 y.o. obese and diabetic woman was admitted to the hospital because of pain and swelling of the lower limb 5 days after a popliteal deep venous thrombosis event. There were skin blistering and edema, but after 12 hours, despite of an aggressive antibiotic therapy, a severe worsening of the local and general conditions happened. The ecography and CT scan showed gas in the soft tissues. She underwent an aggressive fasciotomy and debridement of the lower limb.

She was moved to the ICU, because of an arising septic shock, and submitted to repeated debridements, medication with a negative pressure system and hyperbaric therapy.

Diagnosis was NF, determined by Streptococcus pyogenes, complicated by muscles necrosis.

After 1 month the patient was moved to the Burn Unit, where several escarectomies and autologous skin transplantations were performed.

Results: Three months later the patient started a rehabilitation program and now she walks using a cane.

Conclusions: NF is a life-threatening infection of the soft tissues, a better prognosis is related to an early and aggressive debridement and antibiotics. 
Pediatric Surgery

\section{4}

\section{Obstructive Sleep Apnea Syndrome (OSAS) in Pediatric Patient: The Role of Adenotonsillectomy}

\author{
D. Testa, A. Lecce, G. Marcuccio, G. Castaldo, G. Motta
}

Second University of Study of Naples

Introduction: The Obstructive Sleep Apnea Syndrome (OSAS) is defined as a breathing disorder characterized by prolonged partial (hypopnea) and/or intermittent complete (apnea) upper airway obstruction that disrupts normal ventilation during sleep and normal sleep patterns.

This study was designed to assessthe usefulness of polysomnography (PSG) in the evaluation and management of pediatric patients with mild to severe OSAS, before and after adenotonsillectomy (AT)

Materials and Methods: This is a retrospective investigation. A total of 38 consecutive patients ( 21 male, 17 female), age range 4-10 years, all presenting with clinical manifestations of OSAS.

16 of all patients had PSG-defined OSAS; 12 symptomatic patients with negative polysomnogram; 10 children were studied as controls and, therefore, submitted to watchful-waiting.

The post-operative results were evaluated $3,6,12$, and 18 months after surgery by a clinical-instrumental reevaluation in all the subjects and via PSG in selected patients with severe OSAS. The results obtained were statistically analysed by $\chi^{2}$ test: a " $p$ " value of $<0,05$ was considered statistically significant.

Results: Our findings support the efficacy of AT in the treatment of all clinical manifestations of OSAS in both the groups with or without PSG-defined OSAS.

Conclusion: On the basis of these results, we consider that in all the children with OSAS a polysomnography must be always interpreted with the entire clinical picture in mind, and, therefore, the recommendation for surgery must be individualized, based on both polysomnography and clinical assessments.

\section{5 \\ The Effect of Parental Preference in the Management of Vesicoureteral Reflux in Children}

\section{G. Torino, G. Collura, N. Capozza}

Paediatric Renal Transplant Surgery and Correlated

Patologies Unit, Department of Paediatric Nephrology and Urology, Bambino Gesù Children's Hospital - Institute for

Scientific Research, Rome, Italy

Introduction: The therapeutic options of vescicoureteral reflux (VUR) actually comprise: conservative management (including antibiotic prophylaxis and recently intermittent therapy), endoscopic subureteral injection and open surgery. These treatment options, at least in moderate grade of VUR, are equivalent in terms of prevention of renal damage. We present a new therapeutic protocol for VUR based on parental preference that was analyzed by our previous study.

Materials and Methods: 100 children with VUR grade 3 ( 38 boys and 62 girls, mean age 4 years, age range 1 to 15 years), who had received antibiotic prophylaxis for at least 6 months, were selected to participate in the study.

The parents of these children were provided with detailed information about the three options available for treating VUR, including the mode of action, cure rate and possible complications, and the practical advantages and disadvantages. They were then asked to choose their preferred treatment.

Results: Most parents preferred endoscopic treatment $(80 \%)$, rather than antibiotic prophylaxis $(5 \%)$ or surgery $(2 \%)$; $13 \%$ of parents were not able to choose among the three options. Consequently, a new therapeutic protocol was established and applied, in our unit, according to parental preference.

Conclusions: The endoscopic treatment of VUR grade 3 is strongly preferred by parents of the children.

This data suggests a new algorithm for treating VUR: to patients with persistent reflux (i.e. after a short period of antibiotic prophylaxis), endoscopic treatment should be offered as first-line therapy, whereas surgery would still be recommended for patients in whom endoscopic treatment fails. 
Transplantation

\section{6 \\ Liver Transplantation in HIV-HCV Coinfected Patients: A Single Italian Center Experience in the HAART Era}

\author{
V.R. Bertuzzo, M. Del Gaudio, M.C. Morelli, G. Ercolani, \\ M. Cescon, A. Lauro, A. Cucchetti, M. Zanello, A. Dazzi, \\ A. Amaduzzi, F. Tuci, C. Zanfi, E. Bianchi, F. Coccolini, \\ E. Bigonzi, P. Di Gioia, G.L. Grazi, A.D. Pinna \\ Department of General Surgery and Organ \\ Transplantation, Sant' Orsola-Malpighi Hospital, Bologna, \\ Italy
}

Introduction: It is unclear whether hepatitis $\mathrm{C}$ virus (HCV) coinfection increases mortality in transplanted patients with human immunodeficiency virus (HIV) infection in the era of highly active antiretroviral therapy (HAART).

Materials and Methods: We retrospectively analyzed the outcome of $19 \mathrm{HIV}$ patients submitted to liver transplantation (LT) from November 2004 to January 2009. HIV infection was correlated to other variables and to overall survival after LT.

Results: Among 19 transplanted patients, 17 (89\%) were HCV coinfected, whereas the remaining $2(11 \%)$ were HBV co-infected. While pre-LT HIV-RNA viral load was negative in all the patients, HCV-RNA viral load was positive in 12 (71\%). Hepatocellular carcinoma (HCC) affected 9 patients (47\%). Median CD4 ${ }^{+}$blood count was $426 / \mu \mathrm{l}$ (range 219-876); HAART was administrated to all the patients before LT.

Ten patients (53\%) have dying after LT; main causes of death were HCV recurrence (50\%) and HCC recurrence $(20 \%)$. All the patients which experienced HCV recurrence (9 patients; $47 \%$ ) had pre-LT positive HCV-RNA viral load.

1 - and 3-year overall survival was $73 \%$ and $42 \%$, respectively. Between all the analyzed variables, HCV coinfection and positive pre-LT HCV-RNA viral load are the only two that affect survival, with a 1 - and 3 -year survival of $69 \%$ and $49 \%(\mathrm{P}=0.02)$ and $58 \%$ and $40 \%(\mathrm{p}=0.01)$, respectively.

Conclusion: HIV-HCV coinfection is not an absolute contraindication to OLT. It can be useful to put on waiting list only patients with negative HCV-RNA viral load, to obtain satisfactory long-term results.
387

\section{Long Term Results in Kidney and Pancreas Transplantation: Our Experience}

\author{
A. Broggiato, C. Silvestre, E. Bertin, E.S. Pierobon, \\ A. Soligo, N. Baldan, L. Furian, P. Rigotti \\ S.S.D. Trapianti Rene e Pancreas - Dipartimento di \\ Chirurgia Generale e Trapianti d'Organo Azienda \\ Ospedale-Università di Padova
}

Introduction: Kidney and pancreas transplantation (SPK) is considered the gold standard for patients with diabetes mellitus and associated end stage renal disease. In this paper we present long term results related to patients who recieved SPK at our Center.

Material and Methods: We considered recipients with at least 5-year follow-up. From July 1991 to February 2005 we performed 67 SPK. Fortynine patients had enteric exocrine drainage while 18 had bladder exocrine drainage. Systemic venous drainage was performed in all SPK. Mean donors and recipients age were $28.4 \pm 9.5$ and $40.8 \pm 8.4$ years, respectively. Mean dialysis duration was $27.3 \pm 27.9$ months. Fiftyeight pts were on dialysis and 9 were pre-emptive. Mean diabetes duration was $25.3 \pm 6.8$ years. Immunosuppressive therapy was based on $\mathrm{CyA}+\mathrm{AZA}+$ steroids (12), CyA+MMF + steroids (11) and FK+MMF+steroids (44). 35 pts $(52 \%)$ received induction with thymoglobulin.

Results: After a mean follow-up of $8.6 \pm 3.5$ years, actuarial patient, pancreas and kidney survival rates were $93 \%, 77 \%$ and $86 \%$ respectively at 8 years. Five pts died of heart failure, 1 of cirrhosis HCV-related, 1 of lymphoma and 1 of aspergillosis. During follow up, 18 pancreas were lost ( 5 deaths with functioning graft, 3 vein thrombosis, 5 pancreatitis, 4 chronic rejection, 1 lymphoma) and 12 kidneys were lost (7 deaths with functioning graft, 4 chronic rejection and $1 \mathrm{BKV}$ nephropathy). Mean serum creatinine was $125 \pm 48 \mathrm{umol} / \mathrm{L}$ at 6 years.

Conclusions: SPK allows to achieve optimal long term results in terms of graft survival and function. Death with functioning graft is the main cause of graft loss in the long term. 
Urology

388

Multi Drug Resistant Escherichia Coli Prevalence in the Hospital and Territorial Population

\author{
S. Picozzi, P. Gaia**, M. Tejada**, E. Costa**, L. Carmignani \\ *Urology Department, IRCCS Policlinico San Donato, \\ University of Milan, San Donato Milanese, Milan, Italy, \\ ${ }^{* *}$ Medicine Laboratory Service, Section of Microbiology, \\ IRCCS Policlinico San Donato, San Donato Milanese, \\ Milan, Italy
}

Introduction: ESBL-producing organisms are particularly feared being resistant to all penicillins, cephalosporins, and aztreonam. Aim of this study was to evaluate the prevalence of quinolone-resistant Escherichia coli and/or ESBL producers in the Hospital and territorial population.

Materials and Methods: From January 2008 to December 2009 , all data concerning urine cultures were evaluated. The resistance to quinolones and third generation cephalosporins was tested by the method of microdilution, while confirming the presence of ESBL was assessed by the agar diffusion test.

Results: In 2008, urine cultures were made in 2136 and 1232 patients respectively at territorial and hospital level. Isolation of Escherichia coli, respectively, occurred in 226 samples $(72.7 \%$ of positive urine cultures) and in 112 samples (48.1\%). The presence of quinolone-resistant bacteria was $21 \%$ and $53 \%$. ESBL producing bacteria was $3.5 \%$ and $20.5 \%$. Cross-resistance of ESBL producers to quinolone was present in $100 \%$ and $66 \%$.

In 2009, urine cultures were made in 2396 and 1320 patients respectively at territorial and hospital level. Isolation of Escherichia coli, respectively, occurred in 260 samples (68\% of positive urine cultures) and in 116 samples (46.2\%). The presence of quinoloneresistant bacteria was $21 \%$ and $46 \%$. ESBL producing bacteria was $5.4 \%$ and $20 \%$. Cross-resistance of ESBL producers to quinolone was present in $94.4 \%$ and $70 \%$.

The multi drug resistance is high $(\mathrm{p}<0.01)$ in ESBL producers.

Discussion and Conclusions: These results must be considered in relation to various surgical and urological maneuvers in particular on patients already hospitalized who received broadspectrum antibiotic therapy.
389

\section{Bacterial Sepsis After Prostate Biopsy - A New Perspective}

\author{
L. Carmignani, S. Picozzi, M. Spinelli, S. Dipierro, \\ G. Mombelli, E. Negri, A. Maggioni
}

School of Urology, University of Milan, Genital an Urinary Disease 3rd Year of Course

Chairman: Prof. L. Carmignani; Director of the School:

Prof. A. Maggioni

Introduction: Prostate biopsy still presents complications. Major complications such as urosepsis are uncommon occurring between $1-4 \%$ of the patients.

Materials and Methods: From January 2009 We started a perspective multi-centric evaluation of any patient who underwent prostate biopsy. Prostate biopsy was proposed following EUA guidelines. After the procedure the patients were clinically observed for two hours. A phone call was done after 3, 7, and 30 days to check possible complications, submitting to anyone the same approved questionnaire.

Results: Between January 2009 and March 2010, 367 patients entered this study and 349 (96\%) answered the phone call. Urosepsis occurred in 7 patients (2\%). Mean age was 65 years (60-73 years). All patients received antimicrobial prophylaxis with an oral fluoroquinolone and continued for 3-4 days. The median interval between biopsy and presentation to the emergency room with symptom of urosepsis was 52 hours. Of the 7 patients 6 had positive urine and/or blood cultures, 5 for Escherichia coli and 1 for Aeromonas hydrophila, Aeromonas caviae, and Aeromonas sobria. Patient infected with Escherichia coli developed urosepsis in the first post procedure 48 hours. In four cases, Escherichia coli were classified as MDRO - multidrug-resistant organisms. The patient infected with Aeromonas (sensible to ciprofloxacin) developed urosepsis after 6 days.

Conclusion: When a patient recently undergoes to transrectal prostate biopsy, presents signs of sepsis in the first 48 hours after the examination, an antibiotic resistant Escherichia coli would be suspected and urgent admission in the Emegency Department and early antimicrobial therapy should be started. 


\section{Author Index}

\section{European Surgical Research}

Numbers refer to abstract number
Abate, E. 20

Abbate, V. 356

Acquaro, P. 66, 67

Agnati, M. 293

Agnoletti, V. 181

Agrestini, C. 344, 358

Agrusti, S. 21, 298

Aimone, G. 311

Alberto, B. 352

Aldrighetti, L. 33, 34, 79, 150, $166,167,168$

Alessandro, R. 262

Aletti, G. 339

Alfano, C. $185,186,187,199$

Ali, N. 368, 370, 373, 379

Alifano, M. 230

Allaix, M.E. 22, 117, 140, 142, 143

Allidi, F. 266

Alloni, R. 12

Altilia, F. 68

Amabile, D 17

Amadori, D. 147

Amaduzzi, A. 386

Amato, B. 295, 296

Amato, M. 295

Ambesi-Impiombato, F. 156

Ambrogi, M.C. 240, 245

Ambrosio, M.R. 248

Ancona, E. 43, 93

Andrea, Z. 262

Andreoli, F. 17

Angela, D. 223, 256, 264, 267, 268, 269

Angeletti, G. 236

Angelici, A. 38

Angelini, M. 202

Anile, M. 232

Annacontini, L. 215, 216, 217, $218,219,220,221,222$

Annibale, D. 51, 134

Annoni, M. 312, 313

Ansaloni, L. 181

Antinolfi, P. 369

Antonacci, F. 227

Antonacci, N. 306, 311

Antonelli, G. 60

Apa, D. 24

Aramini, B. 253

Ardito, F. 78, 97

Arena, R. 299

Arezzo, A. 22, 140, 142, 143

Argentino, L. 25, 44
Argnani, D. 254, 255

Arhi, C. 19

Arleo, S. 186, 187, 199

Armaleo, F. 111

Armaro, A. 5

Armenti, A.F. 188

Ascari Raccagni, A. 30

Asioli, S. 30

Assenza, M. 35, 36, 74, 102

Asti, E. 20

Atella, F. 24

Attala, D. 382

Attene, F. 71, 72, 99, 137

Atzeni, M. 189

Audisio, R.A. 175

Avallone, A. 139

AVillari, S. 112

Avino, F. 1

Azas, L. 279

Azzinnaro, A. 175

Baccarani, A. 206

Bacchetti, S. 155, 157

Bachellier, P. 90

Badessi, F. 137

Badiali, G. 353

Bagnariol, F. 54

Baiocchi, G.L. 118

Baisi, A. 263

Balagué, C. 140

Balani, A. 49, 50

Baldan, N. 387

Baldoni, F. 306, 311

Baldoni, G. 46

Ballarin, R. 37

Balzano, A. 195

Bambacaro, P. 151

Bannon, P.G. 284

Barbante, M. 271, 273, 275

Barbaro, B. 153

Barbato, R. 6

Barillà, D. 292

Barlattani, A. 344, 358

Bartoli, A. 149

Bartolucci, P. 258, 259

Bartuli, F.N. 359

Bassetto, F. 200

Basso, A. 135

Basso, N. 65

Bastaroli, E. 18

Baticci, F. 308

Battisti, N. 87

Beccaria, M. 224
Beccarla, A. 112

Bedini, A.V. 251

Belardi, V. 105

Bellantone, R. 129, 152, 153

Bellocchi, G. 323, 331

Beltrami, G. 375

Bencini, L. 119, 144

Benedetti, M. 25, 44

Benedetto, F. 8, 9, 10, 291, 292

Benucci, C. 25, 44

Berardi, G. 63, 132, 133, 301, 302, 303, 304

Berardi, S. 316

Bergossi, L. 94, 95, 96

Berlucchi, M. 325, 326

Bernardi, S. 48

Berretta, P. 5

Bertani, A. 198

Bertelli, R. 94, 95, 96

Berti, P. 28, 58, 130, 229, 230, 231

Bertin, E. 387

Bertocchi, V. 21, 298

Bertoglio, C. 53

Bertolaccini, L. 257

Bertone, F. 18

Bertuzzo, V.R. 386

Bettarini, F. 26, 56

Bettini, D. 314

Bezzi, M. 38, 39, 40

Biagini, C. 381,383

Bianchi, A. 346, 353

Bianchi, E. 386

Bianchini, C. 333

Bianco, E. 327

Bianco, F. 146

Bianco, N. 329

Biazzo, A. 368, 370, 373, 379

Bigonzi, E. 386

Binda, S. 86

Bing, C. $178,179,180$

Biondi, A. 173, 174

Biondi, P. 341, 343

Biscardi, A. 306, 311

Bisceglia, P. 215, 218, 219, 220, 221

Blondeel, P.N. 210

Bna, C. 224

Boaron, M. 227

Boati, S. 27, 308

Bocciolone, L. 339

Boccuzzi, A. 217, 218, 219

Bollero, P. 344, 358
Bomboi, M. 145

Bona, D. 20

Bonadiman, C. 225

Bonaiuto, E. 317

Bonanno, F. 286, 287, 288

Bonanno, P. 271, 274, 275

Bonapasta, S.A. 23

Bonavina, L. 20

Bondanza, G.S. 131

Bonfanti, B. 227

Bongiolatti, S. 248

Boni, L. 85, 108, 120, 121, 123, $124,169,312,313$

Borgogni, V. 46

Bormioli, M. 193, 194, 209

Borrelli, A. 57

Borrelli, M. 3

Borrelli, V. 366, 367

Borriello, C. 363

Bortolotti, U. 7

Bortul, M. 98

Boscaini, G. 203, 206

Boschetti, L. 294

Bottani, G. 18

Botti, F. 113

Bottoli, C. 249, 250

Bottoli, M.C. 224, 225, 226

Bove, M. 382

Bozzetti, A. 345

Braccini, L. 293

Braccio, B. 61, 62

Brafa, A. 190, 191

Bracciand, L. 144

Braidotti, P. 376

Bramanti, C.C. 109,110

Brambilla, D. 271, 272, 275

Brancaccio, A. 321

Brandi, C. 190, 191

Brandolini, J. 28, 58, 130, 229, 230, 231

Brassetti, B.C. 24

Bravi, I. 235

Breda, C. 238

Brescia, A. 63, 132, 133, 301, 302, 303, 304

Broggiato, A. 387

Brunetti, J. 144

Bruni, F. 340

Bruno, B. 134

Bucalossi, M. 46, 127, 297

Bucci, F. 41

Bucci, L. 60

Buccoliero, F. 94, 95 
Bufalari, A. 235

Bulla, A. 195

Buonomo, O. 55, 116

Buonsanto, A. 73

Buri, L. 48

Busoni, C. 270, 282

Buttà, P. 342

Cabras, F. 72

Cacchi, C. 70

Cafarotti, S. 234

Cafiero, F. 164

Cagini, L. 236

Cagli, B. 201

Cagol, P. 155, 157

Calabrese, L. 342, 359

Calabrò, M. 190, 191

Calati, A.M. 263

Caldart, M. 117, 142

Califano, L. 355, 356, 357

Calistri, M. 119, 279

Calmieri, A. 154

Calzolaro, L. 324

Calzoni, C. 187

Camaioni, A. 327,328

Camerino, O.C. 73

Campa, A. 190, 191

Campagna, A. 381, 383

Campana, M. 190, 191

Campanacci, D. 374, 375, 378

Campanacci, L. 379

Campanale, A. 215, 216, 218, 219, 221, 222

Campanelli, G. 21, 298

Campanini, A. 319

Campi, F. 380

Campisi, C.C. 360

Campus, G.V. 208

Cananzi, F.C.M. 173, 174

Cancellieri, F. 311

Cancrini, A. 70

Cancrini, G. 70

Candoli, P. 260

Canta, L. 69, 192

Cantarella, F. 52

Cantore, F. 120, 121, 123, 124

Canzi, P. 14, 319, 320

Canziani, M. 21, 298

Capanna, R. 374, 375, 378

Capolupo, G.T. 12, 29, 154

Caporale, C. 335

Capozza, N. 385

Capozzi, R. 235, 236

Cappai, A. 241

Capretti, P.G. 289

Caprino, P. 316

Caprio, M.G. 365

Caraffa, A. 369

Carcano, G. 86, 107

Carcione, F. 163
Cardone, E. 139, 146

Careddu, L. 11

Carella, G. 8

Cariani, S. 362

Carillo, C. 232

Carmignani, L. 388, 389

Carola, M. 76

Caronna, R. 25, 44

Caronno, R. 289, 290

Carpanese, L. 155, 157

Carpino, A. 88

Carrafiello, F. 359

Carrara, A. 113

Carta, S. 372

Caruso, A. 112

Caruso, S. 26, 56, 141, 151

Casabianca, E. 270, 282

Casacalenda, A. 6

Casadei, R. 147

Casadio, P. 340

Casali, C. 239

Casalino, A. 271, 272, 274, 275

Casarotto, R. 225

Casciola, L. 149

Cascioli, I. 44

Cassanelli, N. 233, 252

Cassinotti, E. 120, 121, 123, 124

Castagnola, M. 131

Castaldo, G. 384

Castano, P. 85, 312, 313

Castelnuovo, P. 332

Castrucci, G. 293

Cataldo, F. 184

Catalini, G. 73

Catena, F. 181

Catena, M. 33, 34, 79, 150, 166, 167,168

Caterino, M. 156

Cautero, N. 37

Cavaliere, D. 1, 31, 32, 122, 147, 148,181

Cavaliere, F. 160

Cavallaro, G. 299

Cavallesco, G. 249, 250

Cavalli, M. 21, 298

Cavallina, G. 119

Cavallini, N. 294

Cavallini, R. 94, 95

Cavazzoni, E. 52

Cavicchi, O. 322

Cazzato, G. 162, 336

Ceccaccio, L. 190, 191, 297

Ceccarelli, G. 149

Ceccarelli, S. 235, 236

Ceccaroni, M. 338, 340

Ceccolini, M. 147

Ceccolini, V. 185, 199

Ceccone, A. 213

Cecere, D. 276

Ceci, F. 300
Celentano, V. 60

Celiento, M. 7

Centonze, L. 102

Ceribelli, C. 149

Cerulli, G. 369

Cerullo, G. 141

Cesari, E. 380

Cescon, M. 386

Chapelier, A. 28

Checcoli, E. 333

Chello, M. 6

Chiappa, C. 85

Chiappetta, M. 234

Chiara, O. 27, 308

Chiarelli, M. 66, 67

Chirletti, P. 25, 44

Chiti, E. 279

Chiummariello, S. 187, 199

Cianchi, F. 87

Ciardiello, F. 243

Cicconetti, F. 258, 259

Ciciliot, M. 361

Ciciriello, M.B. 366, 367

Ciferri, E. 131

Cimbanassi, S. 27

Cioppa, T. 178, 179, 180

Cipollone, I. 29, 154

Cipriani, F. 33, 34, 79, 150, 166, 168

Cipriani, R. 205, 210, 211

Cipriano, M. 65

Claudio, M. 352

Clemente, N. 306, 311

Clementini, M. 344, 358

Cobellis, G. 349

Coccolini, F. 181, 386

Coglitore, A. 108

Colace, R. 88

Colasanto, G. 127

Colaut, F. 261

Coletta, S. 76

Collura, G. 385

Colombo, D.G. 193, 194

Colombo, E. 120, 121, 123, 124

Colombo, G. 209

Colombo, L. 351

Coloni, G.F. 232

Comel, A. 226

Compagna, R. 295, 296

Compiani, E. 280

Confalonieri, G. 66, 67

Contessini-Avesani, E. 113

Conz, V. 333

Coppola, A. 29, 154

Coppola, R. 12, 29, 154

Coratti, A. 46, 127

Coratti, F. 46, 127, 297

Corinaldesi, G. 346

Corona, A. 189

Corso, G. 26, 56, 151
Coscia, V. 294

Cosimelli, M. 155, 156, 157

Costa, E. 388

Costa, M. 66, 67, 335

Costantini, M. 43, 93

Costantino, B. 202

Cotellese, R. 171

Cotroneo, G. 202

Covino, E. 6

Cozza, V. 35, 36, 74

Cremona, F. 139

Cresti, R. 135

Crocetti, D. 36, 74, 310

Crucitti, A. 129, 152, 153

Crucitti, P. 29, 154

Cucchetti, A. 386

Cucinotta, F. 111

Cusumano, G. 234

Cuviello, A. 135

D'Acapito, F. 31, 32, 122, 148

d'Adamo, V. 125, 126

Daddi., N. 235

Dadomo, V. 169

D’Agostino, G. 319

D’Alessandro, G. 360

D’Alessandro, R. 160

D’Alessandro, V. 171

Dallan, I. 320

Dall'Oglio, A. 63, 132, 133, 301, 302, 303, 304

D'Amato, G. 129

Damiani, V. 323, 331

D’Amico, G. 37

D’amico, S. 178, 179, 180

D’Amore, A. 258

D'Amore, L. 300

D’Aniello, C. 190, 191

d'Annibale, M. 156

D’antonio, A. 186

D’Arpa, S. 214

D'Ascoli, B. 41

Dattola, A. 10

David, G. 86, 169

Davini, F. 245, 265

Davoli, F. 233, 252

Dazzi, A. 386

De Berardis, B. 106

De Biasi, S. 355

De Caridi, G. 9

De Donno, G. 224

De Filippis, A.F. 237, 238

De Franciscis, S. 146

De Giacomo, T. 232

De Iaco, P. 181

De Luca, A. 53

De Magistris L. 295

De Maio, G. 41

de Manzini, N. 47, 48, 49, 50, 128 
De Martino, A. 46, 127

De Nisco, C. 137

De Nittis, G. 290

De Porcellinis, S. 6

De Rango, P. 271, 275

De Ruvo, N. 37

De Santis, G. 203, 206

De Silva, G. 131

De Simone, M. 160, 178, 179, 180

De Stefano, A. 141

De Toma, G. 299

De Vito, A. 14, 319

De Vito, D. 295, 296

DeCaridi, G. 291

Degrate, L. 54

Del Bosco, A. 86

Del Gaudio, M. 91, 92, 386

Del Vecchio, D. 200

dell' Antonio, A. 3, 128

Dell'Amore, D. 254, 255

Dell'isola, C. 17

Delrio, P. 139, 146

Denti, S. 100

Deraco, M. 160

D’Ermo, G. 299

Dessena, L. 195

Dessy, L.A. 188

Dezzi, C. $38,39,40$

Di Bartolomeo, R. 5, 11

Di Bella, G. 8

Di Benedetto, F. 37

Di Carlo, C. 337

Di Chiara, F. 228, 238

Di Cosimo, C. 16

Di Filippo, F. 160

Di Francesco, A. 351

Di Fratta, E. 43

Di Giacopo, G. 106

Di Gioia, P. 91, 92, 386

Di Giorgio, L. 377

Di Giuseppe, M. 120, 121, 123, 124

Di Marco, L. 5

Di Mare, G. 56

Di Mare, M. 277

Di Martino, M. 26, 56, 151

Di Martino, N. 61, 62

Di Mauro, G. 115

Di Palo, S. 167

Di Pietrantonio, D. 122, 181

Di Pietro, A. 183, 184

Di Prima, E. 239

Di Sabatino, D. 15

Di Saverio, S. 306, 311

Di simone, M.P.L. 362

Di Somma, C. 164

Di Venere, B. 41

Dieli, F. 214

Digilio, E. 332
Dinatale, G. 25, 44

Dini, P. 240

Dionigi, G. 85, 86, 108, 169, 312, 313

Dionigi, R. 85, 86, 169, 312, 313

Dipierro, S. 389

Dipietrantonio, D. $31,32,148$

Diso, D. 232

Dolci, G. 233, 252

Domenico, L. 223, 256, 264, 267, 268, 269

Donato, R. 8

Dondas, A. 30

Donfrancesco, A. 135

Donini, A. 52

Dorigo, W. 277, 278, 280

D’Ovidio, F. 253, 260

Droghetti, A. 224, 225, 226

Drudi, D. 94, 95, 96

Dubenec, S.R. 283, 285

D’Ugo, D. 173, 174

Eberspacher, C. 15,16

Edelman, J. 284

Elia, E. 52

Elia, S. 55

Emanuel, C. 51

Ercolani, G. 386

Eretta, C. $80,81,82,83,84$

Errani, C. 368, 370, 373, 379

Esposito, A. 42

Estollere, C. 112

Evoli, A. 234

Fabbri, L. 147

Fabbri, N. 94, 95, 96, 379

Fabio, D.A. 262

Fabris, B. 48

Fabris, L. 43

Fabrizio, T. 176

Faccani, E. 94, 95, 96

Faenza, M. 208

Faiella, E. 161, 162, 163, 336

Falasconi, M.C. 147

Falchero, F. 361

Falciani, C. 144

Famà, F. 109, 110, 111, 112

Famiglietti, F. 125, 126

Fanello, G. 25, 44

Fanti, S. 212, 373

Fanucchi, O. 240

Fappiano, F. 295, 296

Fara, G. 204

Farace, F. 204, 208

Faralli, M. 330

Farelli, F. 25, 44

Fargion, A. 278

Farinon, A.M. 17

F.Buccoliero, Cavallini, 96

Fechner, S. 246
Fedele, L. 218

Federico, R. 228, 262

Felice, P. 354

Ferla, G. 34, 167, 168

Fernandez, I.J. 322

Feroci, F. 57

Ferrando, V. 170

Ferrara, F. 173, 174

Ferrara, S. 357

Ferrari, F. 13, 88

Ferraro, S. 209

Ferrata, P. 372

Ferravante, P. 76

Ferreli, F. 332

Ferrucci, P.F. 183, 184

Ferruzzi, L. 260

Festa, F. 117, 125, 126, 142, 143

Fianchini, G. 356

Filippi, N. 241

Finazzi, R. 33

Finotti, E. 93

Fiore, F. 155, 157

Fiorelli, A. 242, 243

Fiorentini, G. 178, 179, 180

Fiorini, E. 241

Flauti, G. 26, 141

Florio, P. 16

Fogacci, T. 2

Folesani, G. 11

Folliero, C. 114, 317

Forcione, A.R. 42

Forestieri, P. 363, 364, 365

Formato, A. 364, 365

Formicola, F. 337

Formisano, C. 337

Fornaro, R. 101

Forte, A. 39

Fortellizze, D. 342

Fortina, M. 372

Fortiparri, S. 227

Framarini, M. 31, 32, 122, 148, 181

Francesca, C. 262

Francesca, T. 223, 264

Franceschi, A. 361

Francesco, F. 134

Francesco, S. 223, 256, 264, 267, 268, 269

Francioni, F. 232

Franco Stella, A.B. 233, 252

Frascio, M. 175

Frattini, F. 108, 169, 312, 313

Frenguelli, A. 324, 330

Frenos, F. 374

Frisoni, G. 2

Furian, L. 387

Fusco, A. 225

Fusco, G. 261

Fusetti, M. 335
Gahir, J. 19

Gaia, P. 388

Galati, D.M. 196

Galbiati, D. 138

Galla, S. 331

Gallazzi, M. 244

Galleano, R. 361

Galli, M. 290

Gallo, F. 343

Gambacorta, M.A. 153

Gambale, G. 1

Gandini, S. 183

Ganio, E. 64

Garancini, M. 54

Garbarini, A. 22

Garelli, E. 249, 250

Gargano, V. 337

Gargiulo, S. 363

Garlaschi, D.A. 193

Garofalo, A. 160

Garufi, C. 156

Garulli, G. 28, 58, 130, 229, 230, 231

Gasbarro, V. 293, 294

Gasparre, A. 286, 287, 288

Gaudio, M. 147

Gavioli, M. 159

Gazzabin, A. 297

Gazzabin, L. 46, 127, 297

Gazzotti, F. 181

Gelati, C. 211

Genco, A. 65

Gentilini, O. 4

Gerosa, E. 18

Gerunda, G.E. 37

Gervasi,R. 114

Gervasoni, C. 351

Ghermandi, C. 181

Ghirardelli, L. 167

Ghiribelli, C. 244

Giacchi, F. 281

Giaccone, C. 117

Giacomel, G. 3, 47, 48, 49, 50, 128

Giacomelli, E. 280

Giampalma, E. 155, 157

Gianetta, E. 101, 175

Giangrande, D. 72, 137

Giangrande, G. 71

Giannarelli., D. 160

Giarratana, S. 245

Giavarini, L. 120, 121, 123, 124

Giglio, M.C. 60

Gigliofiorito, P. 201

Ginanneschi, U. 3, 47

Gioffrè Florio, M.A. 109, 110, 111,112

Giorgetti, P.L. 270, 282

Giorgini, E. 306, 311

Giovanetti, M. 136

Author Index

Eur Surg Res 2010;45:158-307 
Giovanni, A. 134

Giovanni, B. 352

Giovanni, Q. 165

Giraudo, G. 117, 125, 126

Giudice, M. 332

Giuffrè, M. 366

Giulia, G. 217

Giuliante, F. 78, 97

Giuricin, M. 3, 48, 49, 50

Giuseppe, G. 267

Giuseppe, M. 228, 262

Giustacchini, P. 152

Givigliano, F. 246, 247

G.Nisi, Campana, 191

Golfieri, R. 155, 157

Gordini, G. 311

Gossetti, F. 300

Gotti, G. 244, 248

Granai, A.V. 55

Granato, F. 244, 248

Grande, M. 17

Grani, G. 28, 229, 230, 231

Granone, S.P. 234

Grassi, R. 214

Grassia, M. 61, 62

Grasso, R.F. 161, 162, 163, 336

Grazi, G.L. 91, 92, 386

Graziano, P. 134

Graziosi, L. 52

Gregori, M. 23

Grieco, M. 197, 215, 217, 220

Griggio, L. 135

Grillone, G. 53

Grimaldi, L. 190, 191

Grossi, U. 129, 152, 153

Grossi, W. 249, 250

Guaglio, M. 54

Guerra, F. 36, 310

Guerrieri, M. 75

Guerriero, M. 316

Guerrini, G.P. 37

Guidotti, A. 277

Guillou, M. 283

Gullà, M. 324, 330

Gurrado, A. 77, 104, 318

Guzzetti, E. 33, 150, 167

Harris, J.P. 283, 285

Harvey, M. 19

Iacobelli, L. 364

Iacuzio, L. 239

Iannelli, E. 55

Ianni, R. 276

Ibrahim, T. 147

Idone, F. 198

Iemmolo, R.M. 37

Iera, M. 185, 187, 199

Igor, M. 134

Imbriglio, G. 106
Imparato, L. 377

Imperatore, M. 101

Innaro, N. 59, 317

Innocenti, B. 369

Innocenti, P. 171

Ippolito, M. 374, 375, 378

Isernia, G. 273, 274

Izzo, F. 155, 157

Jeremy, R. 284

Kadirkamanathan, S. 19

Kaur, G. 283

Kawamukai, K. 227

Kosuta, M. 47

La torre, F. 36

Lacroce, G. 42

Laface, L. 20

Lambert, Y. 56

Lanaia, A. 115

Lancerotto, L. 200

Landi, S. 381, 383

Landoni, F. 339

Langella, M. 201

Langella, V. 185

Lanza, R. 23

Laperuta, P. 243

Laporta, R. 202

Laurenti, F. 376

Lauro, A. 386

Lazzara, F. 101

Lazzaro, F. 59, 114

Lazzi, S. 248

Lecce, A. 321, 384

Lelli, B. 144

Lembo, F. 215, 219, 221, 222

Lenti, M. 273

Lentini, S. 8, 9, 10, 291, 292

Lenzi, E. 57

Leoncini, L. 248

Leone, A. 5

Leotta, A. 169, 312, 313

Leporati, A. 263

Lerose, M.A. 317

Lettieri, C. 226

Leuratti, L. 362

Libretti, L. 251

Lijoi, F. 381, 383

Liparulo, V. 232

Lissidini, G. 4, 77, 104, 318

Liuzza, C. 214

Lizio, G. 346,354

Llange Ayala, K. 15

Lo Gatto, M. 127

Lococo, F. 234

Lombardi, M. 24

Lombardo, F. 16

Longari, F. 324

Longo, B. 202
Longo, C. 43

Longo, F. 29, 154

Lore, B. 342, 359

Lorenzoni, A. 374, 375, 378

Loschi, P. 203, 206

Lovotti, D. 289

Loy, M. 228, 237

Luca, M. 165

Lucchi, A. 28, 58, 130, 229, 230, 231

Lucchi, M. 245, 265

Lucescu, I. 90

Lucia, B. 228, 262

Luciani, F. 359

Luciano, G. 233, 252

Lucisano, A.M. 59, 114

Luglio, G. 60

Luigina, G. 51

Luperto, M. 54

Luppi, G. 161, 162, 163, 336

Lusini, M. 6

Luzzi, L. 244, 248

Macchia, M.T. 346

Macchitella, Y. 141

Madanas, I. 342

Maffi, F. 130

Maffia, R. $185,187,199$

Maggio, C. 235

Maggioni, A. 339, 389

Maglio, R. 63, 132, 133, 301, 302, 303, 304

Magnani, C. 130

Maini, C.L. 155, 157

Maiorella, A. 216, 222

Makovac, P. 177

Malafronte, L. 330

Malara, C. 112

Malatesti, R. 46, 127, 297

Mallio, C. 161

Mancini, R. 155, 156, 157

Mandolfino, F. 101, 175

Mandrioli, M. 253, 260

Manfredelli, S. 38, 39, 40

Manfrini, M. 368, 370

Manfuso, A. 355

Mangiavacchi, M. 372

Maniscalco, P. 249, 250

Mansi, L. 242

Manzelli, A. 116

Manzo, A. 2

Marano, L. 61, 62

Marazzi, A. 159

Marcantoni, A. 322

Marchetti, C. $346,347,353,354$

Marco, G. 51

Marco, S. 262

Marcuccio, G. 321, 384

Marcuccio, L. 15, 42

Marenco, D. 131
Marengo, M. 212

Marfisi, C. 314

Margaritora, S. 234

Mari, F.S. 63, 132, 133, 301, 302, 303, 304

Maria Tenconi, S. 120

Mariani, F. 26, 56, 141, 297

Marina, L. 51

Marini, G. 335

Marino, M. 37

Maritato, F. 80, 81, 82, 83, 84

Marongiu, F. 195

Marrelli, D. 26, 56, 141, 151

Marrosu, A. 71, 72, 99, 100, 137

Marsanic, P. 158

Martelli, A. 335

Martina, S. 64

Martinelli, F. 15

Martinoli, C. 183

Marucchini, A. 273

Marulli, G. 237, 238

Marzano, E. 90

Marziani, F. 235

Maselli, R. 65

Masoni, L. 63, 132, 133, 301, 302, 303, 304

Massa, S. 68

Massara, M. 8, 10, 291, 292

Massariello, D.N. 216, 217, 220

Mastroluca, E. 15, 16, 42

Matera, D. 374

Matticari, S. 279

Mattioli, S. 253, 260

Mauro, A. 159

Maxia, S. 204

Mazzari, A. 129, 152, 153

Mazzei, M.A. 151

Mazzei, S. 66, 67

Mazzeo, P. 173, 174

Mazzocchi, M. 188

Mazzoni, S. 353

Meacci, E. 234

Medri, M. 212

Mele, C. 78

Melfi, F. 266

Menconi, G.F. 247

Menghi, A. 23

Menghi, R. 129

Mengozzi, M. 254, 255

Menichetti, S. 144

Menichini, G. 186

Meniconi, R.L. 25, 44

Meraviglia, S. 214

Mercatali, L. 147

Mercuri, M. 368, 370, 373, 379

Merlicco, D. 68

Merlini, M. 351

Messina, G. 242, 243

Metere, A. 42

Mezzetti, G. 70 
Miccini, M. 23

Michele, L. 223, 256, 264, 267, 268, 269

Micheletti, S. 147

Miegge, A. 22

Milillo, A. 63, 132, 133, 301, 302, 303, 304

Mimmo, A. 37

Minelli, L. 340

Modafferi, A. 10, 292

Modini, C. 74, 102, 105, 309, 310

Moglia, P. 289

Moio, M. 69, 192

Molea, G. 69, 192

Molini, E. 324

Mombelli, G. 389

Monacelli, M. 235

Monda, A. 363, 364, 365

Monesi, M 371

Monsellato, I. 160

Montalti, R. 37

Montecalvo, A. 6

Montemari, G. 323, 331

Montesi, M. 2

Montevecchi, F. 320

Monteverde, M. 254, 255

Montoli, M. 241

Morandi, U. 239

Morelli, E. 151

Morelli, M.C. 386

Moretti, R. 119, 144

Morganti, V. 345

Morgillo, F. 243

Morino, M. 22, 117, 125, 126, 140, 142, 143

Morlupi, A. 344

Morolli, F. 333

Morselli, P.G. 205, 207

Mosaner, W. 225

Mosca, S. 52

Motta, G. 321, 384

Murabito, L.M. 110, 112

Muriana, G. 224, 225, 226

Muroni, M. 70

Musmeci, S. 4

Mussa, A. 158

Mussa, B. 158

Mussi, A. 240, 245, 265, 266

Muzi, G.M. 17

Muzzeddu, G.P. 204

Nagliati, C. 49,50

Nanni", C. 373

Napolitano, L. 171

Nappi, C. 337

Nardella, D. 197

Nardi, M. 377

Nasti, A.G. 41

Natalini, G. 159
Negosanti, L. 205, 207, 210, 211 , 213

Negri, E. 389

Negro, P. 300

Nejad, P. 205, 207, 211, 213

Nencioni, M. 20

Neri, A. 26, 56, 141

Nezzo, M. 161, 162, 163, 336

Nicola, P. 165

Nicolai, G. 342, 359

Nicotra, S. 43, 228, 237

Nisi, G. 190

Noceti, A. $80,81,82,83,84$

Novelli, E. 64

Nunziatini, R. 147

Nuzzo, G. 78, 97

Oboldi, D. 147

Odero, A. 270, 282

Ondina, P. 223

Oranges, C.M. 207

Oreggia, B. 113

Origi, M. 67

Orlandino, G. 176

Orlando, G. 299

Orsaria, P. 55

Orsenigo, E. 167

Pacchioni, L. 203, 206

Pace, U. 146

Pacelli, F. 316

Pacifico, F. 76

Padovani, F. 319

Pafundi, P. 88, 114

Paganelli, M. 150, 168

Pagliardi, F. 360

Pagliuca, C. 144

Pala, C. 100

Paladini, P. 380

Palazzolo, V. 351

Paliogiannis, P. 71, 72, 100, 137

Palmisano, S. 48, 49, 50

Palo, S. 205, 207, 213

Palombi, L. 16

Palumbo, F. 316

Panaro, F. 90

Pancaldi, A. 63, 132, 133, 301, 302, 303, 304

Pandolfi, C. 358

Pangrazi, P.P. 198

Panico, F. 203, 206

Pantaleo, A. 11

Paolo, B. 165

Paoloni, A. 73

Paparelli, C. 299

Paperetti, L. 278

Papini, F. 25, 44

Parisi, D. 218, 219, 220, 221, 222

Parlani, G. 272

Pasqualini, M. 145
Passafiume, F. 35, 74

Passari, G. 9

Pata, F. 13

Pataia, E. 185, 186, 199

Patella, M. 232

Patrick, P. 90

Patriti, A. 149

Patrizi, A. 75

Patrizi, I. 75

Pattaro, G. 155, 156, 157

Paulatto, L. 49, 50

Pavicevic, J. 5

Pecchielan, A. 43

Pecori, B. 139

Pecoriello, R. 235, 236

Pedone, A. 206

Pedrazzani, C. 141, 151

Pedruzzi, B. 325, 326

Peiretti, M. 339

Pellegrino, G. 346, 354

Pellerito, R. 73

Pelusi, G. 340

Pepi, P. 225

Perello, P. 191

Perello, R. 190

Perigli, G. 87

Perna, S. 249, 250

Perone, M. 364

Perotti, S. 175

Perretti, B. 286, 287, 288

Perri, P. 160

Persiani, P. 382

Persiani, R. 173,174

Persichetti, P. 201

Pertile, D. 164,170

Pesucci, B. 323

Petrella, E. 147

Petrella, G. 55, 116, 203, 206

Petrillo, M. 61, 62

Petrosillo, P. 18

Pettinari, D. 113

Pia, A.N. 256, 268, 269

Piccardo, A. 80, 81, 82, 83, 84

Picchi, A. 266

Piccin, O. 322

Piccinni, G. 77, 104, 318

Picozzi, S. 388,389

Pierobon, E.S. 387

Pignatti, M. 206

Pilati, P. 160

Pilone, V. 363, 364, 365

Pilotti, V. 253, 260

Pindozzi, F. 63, 132, 133, 301, 302, 303, 304

Pini, A. 144

Pinna, A.D. 91, 92, 181, 386

Pinta, M.L. 160

Pinto, E. 26, 56, 141, 151

Pinto, V. 207, 211, 213

Piombino, P. 355, 356, 357
Pipitò, N. 8, 9

Piraccini, E. 1

Pisaniello, D. 76

Pisano, I.P. 99

Piscopo, C. 108

Pistilli, R. 354

Pizzigallo, A. 347

Placanica, P. 111

Poletto, G. 270, 282

Poli, E. 77, 104, 318

Poli, S. 54

Police, A. 15, 16

Polistena, A. 299

Politi, V. 115

Pollicino, A. 109

Ponte, E. 91, 92

Pontrelli, G. 340

Popovich, A. 270, 282

Porcellini, G. 380

Porfidia, R. 61, 62

Porziella, V. 234

Potestio, D. 376

Pratali, S. 7

Pratesi, C. 277, 278, 279, 280

Pratesi, G. 277, 280

Praticò, S. 23

Prezioso, G. 25, 44

Princi, P. 316

Pucci, M. 258, 259

Puce, E. 24

Pugliese, P. 202

Pulighe, F. 71

Pulli, R. 278, 280

Puma, F. 236

Puzziello, A. 114

Qasabian, R. 283, 285

Qirici, E. 87

Quarantotto, F. 249, 250

Quaresima, S. 116

Quintaliani, G. 236

Raffaella, S. 165

Ragusa, M. 236

Raimondi, P. 171

Rainer, A. 6

Rambaldi, P. 242

Rampado, S. 135

Ramponi, F. 283, 284, 285

Ranieri, A. 329

Ranieri, V. 329

Ranucci, G. 78, 97

Raposio, E. 196

Rasovic, O. 11

Ratti, F. 33, 34, 79, 150, 166, 167, 168

Ratto, E.L. $80,81,82,83,84$

Rausei, S. 85, 86, 108, 169, 312, 313

Rauso, R. 349

Author Index 
Raveglia, F. 263

Ravenni, N. 144

Razzaboni, P. 381, 383

Rea, F. 237, 238

Rebecchi, F. 117

Rebuffini, E. 348, 350

Rebusso, A. 228, 237, 238

Reda, G.M. 61, 62

Rencricca, A. 319

Rettori, M. 57

Ribuffo, D. 189

Riccardo, G. 165

Ricci, G. 330

Ricci, M. 147

Ricci, P. 52

Ricci, R. 147

Ricciardi, E. 116

Ricciardulli, T. 309

Riccio, M. 198

Ricella, C. 232

Ridolfini, M.P. 316

Rigamonti, M. 87

Righetti, R. 1

Righini, M.G. 30

Rigotti, P. 387

Rimini, E. 170

Risio, D. 171

Riva, A. 372

Rizzardi, G. 257

Rizzi, A. 263

Rizzuto, A. 88, 89, 172, 317

Rocca, T. 294

Rocchetta, D. 348, 350

Rocci, N. 73

Rocco, A. 323, 331

Roggia, G. 68

Romairone, E. 170

Romani, E. 258, 259

Romano, F. 54

Romano, G. 146

Romano, L. 274

Romano, R. 88, 172

Romelli, A. 66, 67

Rosato, L. 190, 191

Roscio, F. 53

Roseano, M. 177

Rossetti, M.W. 329

Rossi, B. 361

Rossi, C.R. 160

Rossi, F. 86

Rossi, P. 116

Rossi, R. 295, 296

Rosso, E. 90

Rota, A. $80,81,82,83,84$

Rotondo, A. 242

Rotondo, C. 1

Rotundo, A. 19

Rovera, F. 85, 108, 169, 312, 313

Roviello, F. 26, 56, 141, 151

Roviglione, G. 338
Rozen, W. 189

Rubino, C. 195, 204

Rucci, M. 216, 217, 221, 222

Rucci, S. 68

Ruffato, A. 253, 260

Ruffolo, F. 146

Ruggieri, P. 373

Ruggiero, L. 77, 104, 318

Ruggiero, S. 12

Rulli, F. 17

Ruol, A. 43

Ruscelli, P. 94, 95, 96

Ruspi, L. 169

Russillo, G. 25, 44

Ruvolo, V. 194, 209

Saba, L. 189

Sacanna, E. 147

Sacchetti, F. 91, 92

Sacchetti, R. 119

Sacco, E. 36, 310

Sacco, R. 13, 59, 88, 89, 114, 172, 317

Saibene, T. 93

Saino, G. 20

Salvador, R. 93

Salvatori, P. 345

Salvi, M. 28, 58, 130, 229, 230, 231

Salvucci, A. 73

Sammarco, G. 88, 89, 172

Samorani, D. 2

Sangiovanni, V. 349

Sangrigoli, F. 277

Sanguineti, F. 339

Sanna, M. 208

Sanna, S. 254, 255

Sanna, V. 277

Sansoni, I. 12

Santanelli, F. 202

Santella, S. 94, 95, 96

Santi, I. 212

Santini, 154

Santini, M. 242, 243

Sarno, G. 78, 97

Saronni, C. 136

Sarti, E. 341

Sartori, A. 118, 261

Sassaroli, C. 139

Sassu, M.A. 99, 100, 137

Savignani, C. 236

Savini, C. 11

Savini, S. 333

Sbordone, C. 357

Scabini, S. 170

Scaioli, I. 1

Scala, D. 139

Scala, M. 337

Scandroglio, I. 53

Scapin, V. 238
Scarfò, P. 109

Scarsi, B. 209

Scarsi, D.B. 194

Scatizzi, M. 57

Scevola, L. 276

Schettino, M. 61, 62

Schiavon, M. 237, 238

Schiavone, A. 159

Schillaci, O. 55

Schiratti, M. 25, 44

Schirone, S. 68

Sciarra, G. 42

Scioti, G. 7

Scipio, D. 372

Sciuto, R. 155, 157

Scognamillo, F. 71, 72, 99

Scomersi, S. 98

Scordamaglia, R. 170

Scotti, M. 54

Scrocco, D. 76

Scuderi, N. 188

Seccia, M. 45

Selmo, G. 86

Selvaggi, F. 171

Sena, G. 55

Senatore, G. 306, 311

Senescende, L. 46

Senni, G. 24

Serra, P. 147

Serra, V. 37

Serventi, F. 71, 72, 99, 100, 137

Sessa, M. 325, 326

Severi, S. 147

Sgarzani, R. 210

Sguinzi, R. 308

Siani, C. 114

Siboni, S. 20

Sicoli, F. 173, 174

Silvestre, C. 387

Silvestri, V. 272, 273, 274, 309

Simona, M. 352

Simone, M. 327, 328

Simonelli, L. 74, 309

Simonelli, S. 40

Simring, D.V. 283, 285

Sinopoli, 334

Sinopoli, I. 327

Sironi, A. 20

Smerieri, N. 37

Snider, F. 281

Sodano, L. 295, 296, 377

Soggiu, F. 4

Solari, N. 164

Soldati, C. 79, 166

Solfrini, G. 31, 32, 122, 148

Soligo, A. 387

Sollazzo, V. 60

Somma, F. 186

Soteldo, J. 182, 183, 184

Sozzi, D. 345
Spadaccio, C. 6

Spadola, G. 182, 183, 184

Spaggiari, A. 206

Spaggiari, M. 37

Spagnolo, E. 340

Sperduti, I. 156

Spina, D 248

Spina, I. 278

Spinelli, F. 8, 9, 10, 291, 292

Spinelli, G. 348, 350

Spinelli, M. 389

Spinzia, A. 355, 356, 357

Spinzo, G. 203, 206

Spoto, M. 282

Spotti, S. 351

Staals, E. 370, 373

Stabilini, C. 101, 175

Stamatis, G. 246

Stanganelli, I. 212

Stanzi, A. 263

Staudacher, C. 167

Stea, S. 341

Stefani, A. 239

Stephen, M.S. 283, 285

Stilo, F. 10, 292

Sturani, C. 224, 226

Sulpizio, S. 171

Surleti, S. 111, 112

Sussarellu, N. 208

Taddei, S. 280

Tagliabue, F. 66, 67

Tagliani, A. 224

Talamo, M.R. 340

Tang, C.B. 19

Tani, F. 46, 127

Tarantino, B. 102, 309

Tarantino, G. 37

Tarchi, P. 3, 47, 128

Targarona, E.M. 140

Tarquini, R. 60

Tarsitano, A. 347

Tartaro, G. 349

Tassi, V. 235, 236

Tassinari, J. 176

Tassone, D. 205, 207, 211, 213

Tatangelo, F. 139

Tauceri, F. 30, 31, 32, 122, 148, 181

Taurchini, M. 254, 255

Tavaniello, B. 205, 207, 211, 212, 213

Tavolini, V. 272

Tejada, M. 388

Tenconi, S. 244

Tenconi, S.M. 121, 123, 124

Tenna, S. 201

Tenti, G. 14, 320

Teresa, B. 267

Terzi, A. 257 
Tesori, M.C. 15, 16, 42

Testa, D. 321, 384

Testini, M. 77, 104, 318

Testori, A. 182, 183, 184

Theodorakis, E. 376

Tinelli, G. 281

Tinozzi, F.P. 138

Tocchi, A. 23

Töetsch, M. 246

Toia, F. 214

Tomaiuolo, P.M.C. 129, 152, 153

Tommaselli, G.A. 337

Tommasi, C. 119

Toniolo, L. 261

Torelli, F. 61, 62

Torelli, L. 98

Torino, G. 385

Torre, C. 72, 99, 100

Toscano, A. 368, 370, 373, 379

Touloupakis, G. 377

Tozzi, M. 107

Traina, L. 293

Tramontano, S. $363,364,365$

Trani, A. 286, 287, 288

Trevisan, D. 289, 290

Trevisan, G. 30

Trifirò, G. 182

Trignano, E. 71, 99, 188

Trignano, M. 71, 72, 99, 100, 137

Trotta, F. 138

Tucci, G. 17

Tuci, F. 386

Tufo, A. 173, 174

Tugnoli, G. 306, 311

Turri, B. 94, 95, 96

Uggeri, F. 54

Urbani, M. 177
Urbano, V. 39, 40

Ureña, C. 119

Usai, V. 105, 258, 259

Vaccaro, M. 190, 191

Vagliasindi, A. 31, 32, 122, 148

Vaira, M. 160, 178, 179, 180

Valente, D. 348,350

Valente, M. 215, 216, 217, 218, 220, 221, 222

Valentini, M.F. 104

Valentini, V. 153

Valerii, C. 86

Valesini, L. 35, 74, 102, 105, 258, 259

Valitutti, P. 276

Valle, M. 160

Vallicelli, C. 181

Valmasoni, M. 43, 93

Valsecchi, S. 351

van Leuween, B.L. 175

Vandi, F. 28, 58, 130, 229, 230, 231

Vannucci, J. 235, 236

Varrone, F. 127

Vazzana, G. 291

Vellone, M. 78

Velluti, F. 105, 310

Venturini, M. 79, 166

Venturoni, A. 106

Venuta, F. 232

Venza, M. 55

Verdecchia, G.M. 31, 32, 122, 148,181

Vergano, L.B. 371

Veronesi, P. 4

Verrecchia, F. 182, 183, 184

Versace, G. 111
Vescio, G. 88, 89, 172, 317

Vetrone, G. 91, 92

Vettoretto, N. 136

Viarengo, M.A. 300

Vicentini, A. 225

Vicidomini, G. 242, 243

Vicini, C. 319, 320

Vietti, V. 210

Villa, F. 107

Villa, M. 17

Villamaina, E. 363, 364, 365

Villani, C. 377,382

Villani, P. 68

Villani, S. 306, 311

Villari, S.A. 109, 110, 111

Vincenzo, P. 256

Vinci, D. 235

Virzì, S. 160

Vita, M. 234

Vitali, G.C. 182, 183, 184

Viti, A. 265

Viti, C. 327,328

Vitullo, F. 377

Volterrani, L. 151

Voltolini, L. 244, 248

Walter, B. 51

Waugh, R. 283

Weiss, A. 360

White, G.H. 283, 284, 285

Zaccaroni, A. 314

Zanagnolo, V. 339

Zanardi, A. 18

Zanconati, F. 98

Zanello, M. 386

Zanfi, C. 386

Zannetti, G. 205, 212, 213
Zavagno, V. 52

Zavalloni, F. 207

Zefelippo, A. 113

Zerotti, C. 147

Zeuli, M. 156

Zingaretti, N. 174

Zirafa, C. 266

Zittel, F.U. 89

Zobel, B.B. 161, 162, 163, 336

Zoccali, M. 173, 174

Zonta, M. 182

Zucchini, S. 14

Zuin, A. 237, 238 


\section{Erratum}

In abstract No. 134 of the 23rd National Congress of the Italian Society of Young Surgeons [European Surgical Research 2010;45:208] entitled 'Laparoscopic treatment of a carcinoma of the cecum incarcerated in a right groin hernia report of a case', the author's names are:

I. Monsellato, G. Pernazza, G. Alfano, B. Bascone, F. Felicioni, A. D’Annibale. 